\title{
Bioassay and NMR-Guided Isolation of Natural Products from Pacific Marine Invertebrates
}

by

Vidhiya Damodaran

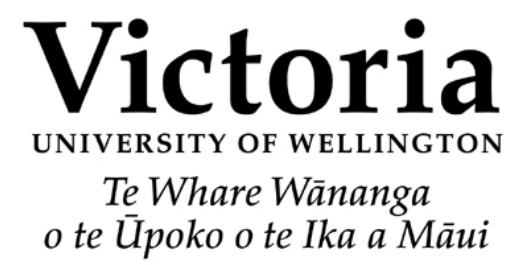

iq

\author{
A thesis \\ submitted to Victoria University of Wellington \\ in partial fulfilment of the \\ requirements for the degree of \\ Master of Science \\ in Chemistry.
}

Victoria University of Wellington 2013 


\section{Abstract}

In the present study, bioassay and NMR approaches were used in combination to guide isolation of two new and three known compounds. An extract library of New Zealand and Tongan marine invertebrates was screened for activity inhibiting the growth of Saccharomyces cerevisiae. From this, 'hits' were identified and validated and three New Zealand sponges and a Tongan ascidian were chosen for bulk extraction. These invertebrates were extracted in methanol and purified using normal and reversed-phase chromatography to isolate the compounds of interest. The isolation of compounds was guided by either inhibitory activity towards $S$. cerevisiae, ${ }^{1} \mathrm{H}$ NMR spectroscopy or a combination of the two.

The known trihydroxylated steroid (14) was isolated from the calcareous sponge Leucosolenia sp. A fatty acid and mixture of phthalate esters were isolated from an unidentified Tongan ascidian. The fatty acid was proposed to be $c i s$-vaccenic acid (13) on the basis of ${ }^{1} \mathrm{H}$ and ${ }^{13} \mathrm{C}$ NMR data. The phthalate ester mixture was not purified further. Work on these two compounds was discontinued due to a lack of activity observed in the $S$. cerevisiae assay and the well-known nature of fatty acids and phthalate esters.

Work was also done on an assay targeting the large mechanosensitive channel (MscL) of bacteria. Some parameters were optimised for this assay, however some work remains to be done. The screening of the extract library of New Zealand and Tongan marine invertebrates using this assay was unsuccessful. More studies to better understand the behaviour of this assay are required, some of which are proposed herein.

The extraction of the New Zealand marine sponge Haliclona sp. yielded one known and two new 3-alkyl pyridinium alkaloid (3-APA) monomers. The structures of these 3-APA monomers-dehydrohaliclocyclin C (58), dehydrohaliclocyclin F (59) and the known haliclocyclin C (20)-were elucidated using a combination of NMR spectroscopy, mass spectrometry (MS) and chemical degradation. This is the first report of a 3-APA cyclic monomer with unsaturation in the alkyl chain being isolated. 3-APAs were identified as the source of inhibitory activity in the S. cerevisiae assay, however, no inhibitory activity was found for 58, 59 and 20 against the clinically relevant fungus C. albicans.

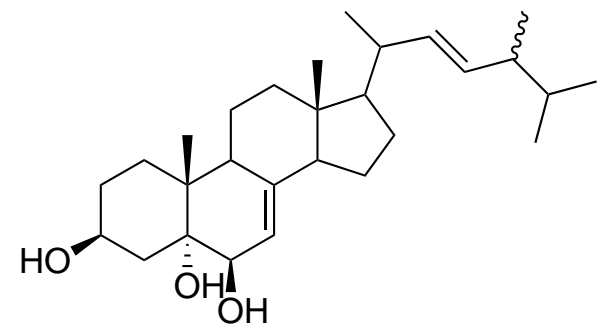

14

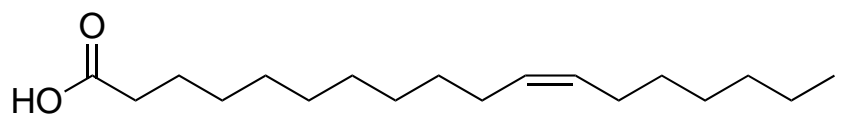

13 


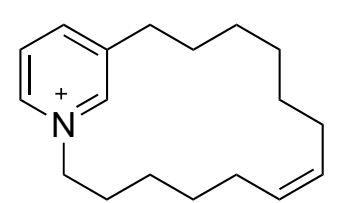

58

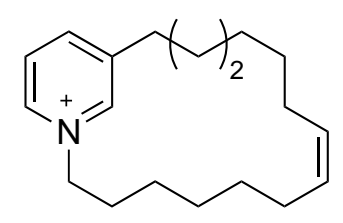

59<smiles>c1cc2c[n+](c1)CCCCCCCCCCCCC2</smiles>

20 


\section{Acknowledgements}

There are many knowledgeable people whom I have met over the course of this work, this thesis would not have been possible without them. I have learnt so much over the last two years and am very grateful to all the people who made this possible.

I would like to thank my supervisor Dr. Rob Keyzers for all of his time, support, guidance, patience, constant positivity and enthusiasm. I could not have done this without all your knowledge and encouragement, thank you for always making time for me. To my lab mates Nathaniel Dasyam, Emma Aitken, Chriselle Braganza, Danica Carter and Laura Walker thank you for making working in the laboratory a fun and sometimes crazy experience! Thanks especially to Nathaniel who taught me all there was to know about our laboratory and never complained, no matter how many times I asked for help. I have appreciated your catch-phrases of support and your humour throughout the past two years, you have been great company in the lab and throughout the constant bickering, music wars, tin foil madness and 'decorating' of benches you have been a great friend.

I am very grateful to Associate Professor Peter Northcote who has been a fountain of knowledge in so many areas. I have enjoyed being surprised and amazed at the extent of subjects of which you appear to have an in-depth understanding. To the Marine Natural Products research group thank you for being great company and offering advice or glassware when I needed it. Particularly, to Dr. Jonathan Singh, I have greatly appreciated all your help with NMR experiments and advice on miscellaneous subjects, you always made yourself available to help me out despite having your own work to do. To Dr. Jacqueline Barber, thank you for helping to get me started when working in the chemical genetics lab I would have been lost without your instruction. I would also like to thank Dr. Joanne Harvey for her advice about oxidative bond cleavage and Professor John Spencer for lending me the reagents required.

Thank you to Dr. Penny Truman, who had the time and patience to teach me about the MscL assay and was available for me to bounce ideas about my latest results off. I am also very grateful to Professor Boris Martinac for allowing me to work on this MscL assay and providing help and advice when it was needed. I would like to thank the Marine Natural Products group at VUW for collecting the New Zealand and Tongan marine invertebrates to be tested. We are especially grateful to Mike Page and Michelle Kelly (NIWA) for providing us with information on the relevant sponges. Furthermore, I would like to thank Emma Aitken, Juliane Bock and Barbara Paul for making up the $3 \times 96$-well plates of the New Zealand and Tongan marine invertebrate library.

A huge thank you to Jason Ryan from Callaghan Innovation for performing the assays against Candida albicans. I am also extremely grateful to Dr. Dave Bellows and Professor Paul Atkinson for allowing me to work in the Chemical Genetics laboratory at VUW and to Christina Roberts, Bede Busby and Namal Coorey for lending a hand when I needed it. To Teresa Gen, Jackie King and Jaime-Anne Elliott thank you for kindly lending me equipment. To my fellow postgraduates, thank you for the advice and fun times. Particularly, to Emma Aitken and Claire Turner who have been working towards their MSc alongside me, thank you for the encouragement, support and late-night company towards the end. Thank you also to Peter Moore for all your advice.

I would like to gratefully acknowledge the Curtis-Gordon Research Scholarship in Chemistry, the Victoria Graduate Award, the Studylink Bonded Merit Scholarship and 
the Victoria Masters (by thesis) Scholarship for providing funding.

To Ved, thank you for your patience, encouragement, love and support that has never wavered. Finally, to my Mum, thank you for the love, support and many meals you have provided me with over the years. You have always been there for me no matter what and I could not have come this far without you. 


\section{Contents}

$\begin{array}{ll}\text { Abstract } & \text { ii }\end{array}$

Acknowledgements iv

Table of Contents vi

List of Figures $\quad x$

List of Schemes $\quad$ xii

List of Tables $\quad$ xiii

Glossary xiv

1 Introduction 1

$1.1 \quad$ Natural Products Chemistry . . . . . . . . . . . . . . . . . . . . . 1

1.2 Marine Natural Products . . . . . . . . . . . . . . . . 2

1.2.1 Marine Sponges . . . . . . . . . . . . . . . . 4

1.3 Natural Products Screening and Isolation . . . . . . . . . . . . . 6

1.3.1 Antifungals ................... 7

1.3.2 Antibacterials ................... 9

1.4 Research Aims . . . . . . . . . . . . . . . . . . . . 10

2 Bacterial Mechanosensitive Channels $\quad 12$

2.1 Discovery of Mechanosensitive Channels . . . . . . . . . . . . . . . 12

2.2 Techniques for Measuring Msc . . . . . . . . . . . . . . . . . . . . . 14

2.3 Distinction of Mechanosensitive Channels . . . . . . . . . . . . . . 15

2.4 Large Mechanosensitive Channels (MscL) . . . . . . . . . . . . . . . 16

2.4 .1 MscL Gene . . . . . . . . . . . . . . . . 16 
2.4 .2 Crystal Structure . . . . . . . . . . . . . . 17

2.4 .3 Mechanism .................... 20

2.5 MscL Bioassay . . . . . . . . . . . . . . . . . . 22

$\begin{array}{lll}3 & \text { Screening } & 24\end{array}$

3.1 Antifungal Assay . . . . . . . . . . . . . . . . 24

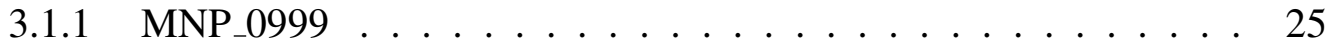

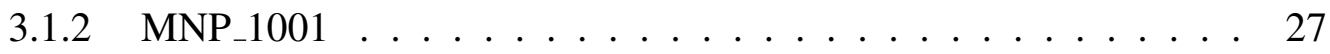

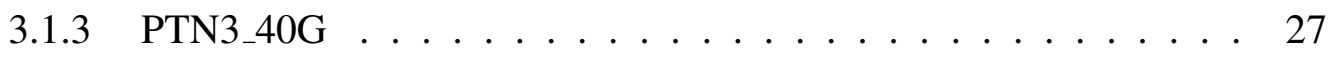

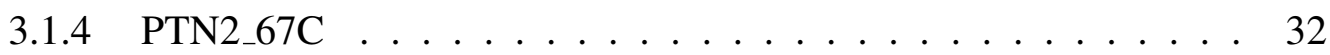

3.2 MscL Assay . . . . . . . . . . . . . . . . . . . . 38

3.2.1 Optimising Parameters . . . . . . . . . . . . . . 39

3.2.2 Testing the Assay . . . . . . . . . . . . . . . . . 48

4 3-Alkyl Pyridinium Alkaloids $\quad \mathbf{5 0}$

4.1 Introduction . . . . . . . . . . . . . 50

4.2 Linear 3 -APAs . . . . . . . . . . . . . . . 51

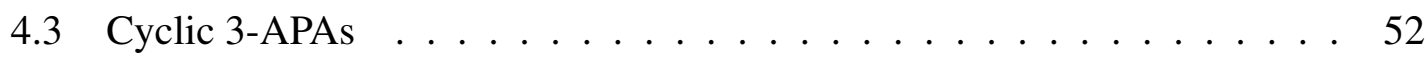

4.3.1 Monomers ................... 52

4.3 .2 Dimers ........................... 54

4.3 .3 Trimers ........................... 56

4.3 .4 Oligomers ...................... 60

4.3.5 Structural Elucidation $\ldots \ldots \ldots 2$

4.43 -APAs Isolated $\ldots \ldots \ldots$. . . . . . . . . . . . . . . . . . 64

4.4.1 Dehydrohaliclocyclin C . . . . . . . . . . . 68

4.4.2 Dehydrohaliclocyclin F . . . . . . . . . . . . 73

4.4 .3 Haliclocyclin C . . . . . . . . . . . . . . 79

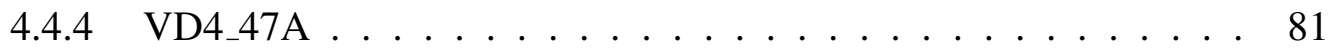


4.4.5 Biological Activity . . . . . . . . . . . . . 82

5 Concluding Remarks $\quad 83$

$\begin{array}{llr}6 & \text { Experimental } & 86\end{array}$

6.1 General Experimental . . . . . . . . . . . . . . . . . 86

6.2 Saccharomyces cerevisiae Assay . . . . . . . . . . . . . . . . 87

6.2.1 Extract Library Screening Protocol . . . . . . . . . . . . . 88

6.2.2 Dose-Response Assay Protocol _. . . . . . . . . . . . . 89

6.3 Candida albicans assay . . . . . . . . . . . . . . . . 90

6.4 MscL Assay . . . . . . . . . . . . . . . . . . . . . . . 90

$6.4 .1 \quad$ Assay Protocol . . . . . . . . . . . . . . . . . . 91

6.5 Isolation of 3-APAs from MNP_0999 . . . . . . . . . . . . . . . . 92

6.5.1 Dehydrohaliclocyclin C and F and Haliclocyclin C . . . . . . . 93

6.5.2 Oxidation of Dehydrohaliclocyclin F . . . . . . . . . . 96

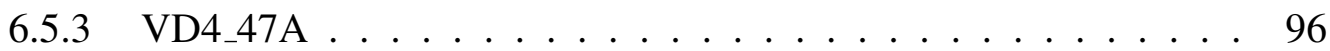

6.6 Extraction of MNP_1001 . . . . . . . . . . . . . . . 97

6.7 Isolation of Compounds from PTN3 $440 \mathrm{G} \ldots \ldots$. . . . . . . . . . 98

6.8 Isolation of Steroids from PTN2_67C $\ldots \ldots \ldots$

A NMR Spectra of Dehydrohaliclocyclin C 102

B NMR Spectra of Dehydrohaliclocyclin F 108

$\begin{array}{lr}\text { C NMR Spectra of Known Compounds } & 114\end{array}$

C.1 Haliclocyclin C . . . . . . . . . . . . . . . . . . . . . . 114

C.2 Steroid VD5_71J . . . . . . . . . . . . . . . 116

C.3 Steroid Mixture VD4_05KLM . . . . . . . . . . . . . 117 
D NMR Spectra of Discontinued Projects

D.1 3-APA VD4_47A . . . . . . . . . . . . . 119

D.2 Fatty Acid VD3_98G . . . . . . . . . . . . . . . 121

D.3 Phthalate Ester VD4_17B . . . . . . . . . . . . . . 123

$\begin{array}{ll}\text { Bibliography } & 125\end{array}$ 


\section{List of Figures}

2.1 Mechanosensitive channels (Msc) in osmotic regulation . . . . . . . . . 13

2.2 Crystal structure of MscL . . . . . . . . . . . . . . . . . . . . . 19

2.3 LPC effect on lipid bilayer . . . . . . . . . . . . . . . . 22

3.1 Surface photograph of MNP_0999 collected off Port Hardy, d'Urville Island, New Zealand . . . . . . . . . . . . . . . . . . . 26

3.2 ${ }^{1} \mathrm{H}$ NMR spectra of VD1_96A and VD1_96B $\left(600 \mathrm{MHz}, \mathrm{CD}_{3} \mathrm{OD}\right) \ldots . . .26$

3.3 Surface photograph of MNP_1001 collected off Port Hardy, d'Urville Island, New Zealand . . . . . . . . . . . . . . . . . . 27

3.4 Surface photograph of PTN3_40G collected at Swallows Cave in Vava'u,

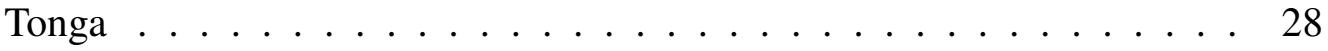

$3.5{ }^{1} \mathrm{H}$ NMR spectrum of VD3_98G $\left(600 \mathrm{MHz}, \mathrm{CDCl}_{3}\right) \ldots \ldots . \ldots . . \ldots$

$3.6{ }^{13} \mathrm{C}$ NMR spectrum of VD3_98G $\left(150 \mathrm{MHz}, \mathrm{CDCl}_{3}\right) \ldots \ldots . \ldots$

3.7 A phthalate ester structure $\ldots \ldots \ldots \ldots \ldots$

$3.8{ }^{1} \mathrm{H}$ NMR spectrum of VD4_17B $\left(600 \mathrm{MHz}, \mathrm{CD}_{3} \mathrm{OD}\right) \ldots \ldots . \ldots . \ldots . . \ldots 2$

$3.9{ }^{13} \mathrm{C}$ NMR spectrum of VD4_17B $\left(150 \mathrm{MHz}, \mathrm{CD}_{3} \mathrm{OD}\right) \ldots \ldots . \ldots . \ldots 32$

3.10 Surface photograph of PTN2_67C collected at Cape Karikari, New Zealand 33

$3.11{ }^{1} \mathrm{H}$ NMR spectrum of VD5_71J $\left(600 \mathrm{MHz}, \mathrm{CD}_{3} \mathrm{OD}\right) \ldots \ldots$

3.12 Key COSY and HMBC correlations establishing the A and B ring structure of steroid VD5_71J . . . . . . . . . . . . . . . . 37

3.13 Key COSY and HMBC correlations providing evidence for the $\mathrm{C}$ and $\mathrm{D}$ ring structure of steroid VD5_71J . . . . . . . . . . . . 37

3.14 Key COSY and HMBC correlations establishing the side chain and structure fragments of steroid VD5_71J . . . . . . . . . . . 38

3.15 Carboxyfluorescein $(\mathrm{CF})$ buffer dilution curve . . . . . . . . . . . 39

3.16 Carboxyfluorescein (CF) buffer dilution curve testing the number of flashes used . . . . . . . . . . . . . . . . 40 40

3.17 Control liposomes stability in different solvents . . . . . . . . . . . . 43

3.18 Control liposomes stability towards crude extracts . . . . . . . . . . . 43 
3.19 Effect of LPC on MscL and control liposomes at varying concentrations

3.20 Effect of liposome concentration on fluorescence change caused by LPC .

4.1 Taxonomic classification to genus level of Haplosclerid sponges that 3-APAs have been isolated from . . . . . . . . . . . . . . . 51

4.2 Compound numbering of cyclic 3-APAs . . . . . . . . . . . 62

4.3 Examples of Hofmann elimination, onium reaction and McLafferty rearrangement fragmentations . . . . . . . . . . . . 6 64

4.4 Key COSY and HMBC correlations establishing the 3-APA pyridinium substructure of dehydrohaliclocyclin $\mathrm{C} \ldots \ldots \ldots \ldots$

4.5 Key COSY and HMBC correlations establishing the structure of dehydrohaliclocyclin $\mathrm{C} \ldots \ldots \ldots \ldots$. . . . . . . . . . . . . . . .

4.6 ${ }^{1} \mathrm{H}$ NMR spectrum of dehydrohaliclocyclin $\mathrm{C}$ showing expanded views of important regions $\left(600 \mathrm{MHz}, \mathrm{CDCl}_{3}\right) \ldots \ldots . \ldots 71$

4.7 2D NOESY NMR spectrum of dehydrohaliclocyclin $\mathrm{C}$ with an expanded view of the weak alkene proton correlations $\left(600 \mathrm{MHz}, \mathrm{CDCl}_{3}\right) \quad \ldots \quad \ldots 72$

4.8 Expanded view of the alkene correlations in the coupled HSQC of dehydrohaliclocyclin $\mathrm{C}\left(600 \mathrm{MHz}, \mathrm{CDCl}_{3}\right) \ldots \ldots . \ldots 72$

4.9 Homonuclear decoupled ${ }^{1} \mathrm{H}$ NMR spectrum of dehydrohaliclocyclin C . . 73

4.10 Key COSY and HMBC correlations establishing the 3-APA pyridinium substructure of dehydrohaliclocyclin $\mathrm{F} \ldots \ldots \ldots \ldots$

4.11 Key COSY and HMBC correlations of the alkene substructure of dehydrohaliclocyclin $\mathrm{F} \ldots \ldots \ldots \ldots . \ldots \ldots 7$

4.12 1D TOCSY array irradiating the 20-position proton resonances $(600 \mathrm{MHz}$,

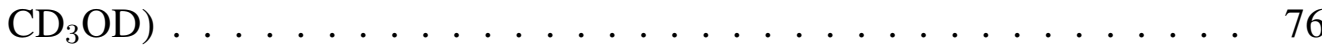

4.13 1D TOCSY array irradiating the 7-position proton resonances $(600 \mathrm{MHz}$,

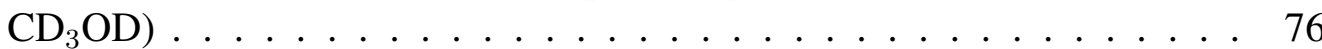

4.14 1D TOCSY array irradiating the alkene proton resonances $(600 \mathrm{MHz}$,

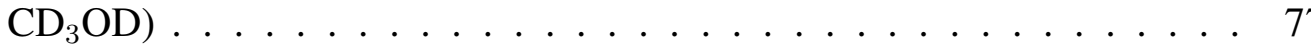

4.15 Homonuclear decoupled ${ }^{1} \mathrm{H}$ NMR spectrum of dehydrohaliclocyclin F . . 77

4.16 Expanded view of the alkene correlations in the coupled HSQC of dehydrohaliclocyclin $\mathrm{F}\left(600 \mathrm{MHz}, \mathrm{CD}_{3} \mathrm{OD}\right) \ldots \ldots . \ldots 78$

4.17 Key COSY and HMBC correlations establishing the 3-APA pyridinium substructure of haliclocyclin $\mathrm{C} \ldots \ldots \ldots \ldots$ 


\section{List of Schemes}

3.1 Isolation procedure for unsaturated fatty acid (VD3_98G) and phthalate ester (VD4_17B) from the sponge PTN3_40G . . . . . . . . . . . . . 29

3.2 Isolation procedure for the steroid VD5_71J from the sponge PTN2_67C . 35

4.1 Isolation procedure for 3-APAs from the sponge MNP_0999 . . . . . . . 65

4.2 Isolation procedure for the dehydrohaliclocyclins $\mathrm{C}$ and $\mathrm{F}$ and haliclocyclin $\mathrm{C}$ from the fraction VD2_384G that was isolated from the sponge

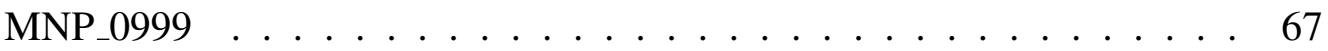

4.3 Isolation procedure for unknown 3-APA (VD4_47A) from the sponge

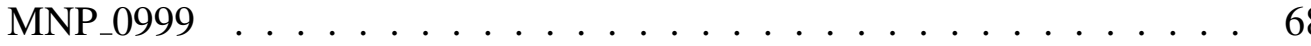




\section{List of Tables}

3.1 Comparison of fluorescence in control and burst CF-containing liposomes 42

4.1 Cyclic Monomer 3-Alkyl Pyridinium Alkaloids . . . . . . . . . . . . . 53

4.2 Cyclic Dimer 3-Alkyl Pyridinium Alkaloids . . . . . . . . . . . . . . . 57

4.3 Cyclic Trimer 3-Alkyl Pyridinium Alkaloids . . . . . . . . . . . . . . . . 60

4.4 Cyclic Oligomer 3-Alkyl Pyridinium Alkaloids . . . . . . . . . . . . . 61

$4.5{ }^{13} \mathrm{C}(150 \mathrm{MHz})$ and ${ }^{1} \mathrm{H}(600 \mathrm{MHz}) \mathrm{NMR}$ data $\left(\mathrm{CDCl}_{3}\right)$ for dehydrohaliclocyclin $\mathrm{C} \ldots \ldots \ldots \ldots 73$

4.6 ${ }^{13} \mathrm{C}(150 \mathrm{MHz})$ and ${ }^{1} \mathrm{H}(600 \mathrm{MHz}) \mathrm{NMR}$ data $\left(\mathrm{CD}_{3} \mathrm{OD}\right)$ for dehydrohaliclocyclin $\mathrm{F} \ldots \ldots \ldots \ldots . \ldots \ldots$

6.1 Yeast Strains Used _ . . . . . . . . . . . . . . . . . . 88 


\section{Glossary}

$\delta$

${ }^{13}$ C NMR

${ }^{1} \mathrm{H}$ NMR

3-APA

AIDS

ATP

br

$\mathbf{C}_{6} \mathbf{D}_{6}$

C18

$\mathrm{CD}_{3} \mathrm{OD}$

$\mathrm{CDCl}_{3}$

CF

$\mathrm{CH}_{2} \mathrm{Cl}_{2}$

CID

COSY

d

DAD

dd

ddd

DDM

Diol

DMSO

$\mathbf{E C}_{50}$

Ec-MscL

Ec-MscS

EDTA

ELSD

EMEA

EPR

ESR

FDA

HEPES

HMBC

HP20/HP20ss

HPLC

HRESIMS

HSQC

IC $_{50}$

IHD

in vitro

in vivo

$J$

kDa

$\mathbf{L D}_{50}$

LPC
Chemical shift(ppm).

Carbon nuclear magnetic resonance.

Proton nuclear magnetic resonance.

3-Alkyl pyridinium alkaloids.

Acquired immune deficiency syndrome.

Adenosine triphosphate.

Broad.

Deuterated benzene.

Octadecyl derivatised silica.

Deuterated methanol.

Deuterated chloroform.

Carboxyfluorescein.

Dichloromethane.

Collision-induced dissociation.

Correlation spectroscopy $\left({ }^{1} \mathrm{H}\right.$ to $\left.{ }^{1} \mathrm{H}\right)$.

Doublet.

Diode array detector.

Doublet of doublets.

Doublet of doublet of doublets.

n-Dodecyl- $\beta$-D-maltopyranoside.

2,3-Dihydroxy-propoxypropyl-derivatised silica gel.

Dimethyl sulfoxide.

Concentration at which $50 \%$ of the population is affected.

The mechanosensitive channel of large conductance found in

Escherichia coli.

The mechanosensitive channel of small conductance found in

Escherichia coli.

Ethylenediaminetetraacetic acid.

Evaporative light scattering detector.

European Medicines Agency.

Electron paramagnetic resonance.

Environmental Science and Research (located in Kenepuru,

Wellington).

United States Food and Drug Administration.

N-2-Hydroxyethylpiperazine-N'-2-ethanesulfonic acid.

Heteronuclear multiple-bond correlation $\left({ }^{1} \mathrm{H}\right.$ to $\left.{ }^{13} \mathrm{C}\right)$.

Poly(styrene-divinylbenzene) stationary support.

High-performance liquid chromatography.

High-resolution electrospray ionisation mass spectrometry.

Heteronuclear single-quantum coherence $\left({ }^{1} \mathrm{H}\right.$ to $\left.{ }^{13} \mathrm{C}\right)$.

Concentration at which $50 \%$ of cell growth was inhibited.

Index of hydrogen deficiency.

In an artificial environment.

In a living organism.

Scalar coupling constant $(\mathrm{Hz})$.

KiloDaltons.

Concentration at which $50 \%$ of the population will die.

Lysophosphatidylcholine in this MscL assay 1-oleoyl-2hydroxy-sn-glycero-3-phosphocholine is used. 
$\mathrm{MeOH}$

MIC

MRSA

MS

MS/MS

Msc

MscK

MscL

MscM

MscS

mult.

MYA

NIWA

NMR

nOe

NOESY

OD

PBS

PC

PCx

ppm

pS

PSDVB

q

Q-TOF

quin

RPM

S

Sa-MscL

Sa-MscL(C $\Delta$ 26)

SC

SDSL

t

Tb-MscL

TLC

TM1

TM2

TOCSY

tt

UV/Vis
Moles per litre.

Multiplet.

Mass to charge ratio.

Acetone.

Acetonitrile.

Methanol.

Minimum inhibitory concentration.

Methicillin-resistant Staphylococcus aureus.

Mass Spectrometry.

Tandem mass spectrometry.

Mechanosensitive channels.

The potassium-dependant mechanosensitive channel.

The mechanosensitive channel of large conductance.

The mechanosensitive channel of mini conductance.

The mechanosensitive channel of small conductance.

Multiplicity.

Malt yeast agar (media).

The National Institute of Water and Atmospheric Research (located in Wellington).

Nuclear magnetic resonance spectroscopy.

Nuclear Overhauser effect.

Nuclear Overhauser enhancement spectroscopy $\left({ }^{1} \mathrm{H}\right.$ to ${ }^{1} \mathrm{H}$ through space).

Optical density.

Phosphate-buffered saline.

Phosphatidylcholine.

Phosphatidylcholine with monosaturated chains of $\mathrm{x}$ carbons.

Parts per million.

Picosiemens.

Poly(styrene-divinylbenzene).

Quartet.

Quadrupole time-of flight.

Quintet.

Revolutions per minute.

Singlet.

The mechanosensitive channel of large conductance found in Staphylococcus aureus.

The mechanosensitive channel of large conductance found in Staphylococcus aureus with a truncation of the last 26 residues of the carboxy-terminus.

Synthetic complete (media).

Site-directed spin labelling.

Triplet.

The mechanosensitive channel of large conductance found in Mycobacterium tuberculosis.

Thin layer chromatography.

First transmembrane helix.

Second transmembrane helix.

Total correlation spectroscopy $\left({ }^{1} \mathrm{H}\right.$ to $\left.{ }^{1} \mathrm{H}\right)$.

Triplet of triplets.

Ultraviolet-visible spectroscopy. 
Victoria University of Wellington.

WNS

White-nose syndrome (in bats).

YPD

Yeast extract peptone dextrose (media). 


\section{Chapter 1}

\section{Introduction}

\subsection{Natural Products Chemistry}

For thousands of years humans have relied on plant sources for medicines. Documents from Mesopotamia, Ancient Egypt, China and India all provide records of the use of plants for medicine. Some plant extracts employed as early as $2600 \mathrm{BC}$ are still in use today, including oil extracted from Papaver somniferum (poppy), which is used in cough medicines. Compounds isolated from poppies are also used as painkillers such as morphine (1) and codeine (2). ${ }^{1,2}$ The discovery of the natural product penicillin in 1928 eventually lead to the 'Golden Age of Antibiotics' from the 1940s to the 1970s. ${ }^{1,3}$ This discovery was also very important as penicillin is still used today to treat some bacterial infections.

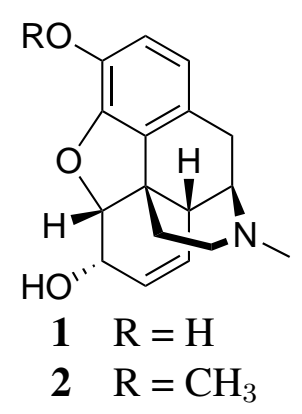

Natural products still play an important role in drug development; between 1981 and 2008 a study of the sources of drugs showed that $63 \%$ of new drugs could be classed as being naturally derived compounds. ${ }^{4,5}$ Furthermore, $68 \%$ of new antibacterial, antifungal, antiviral and antiparasitic drugs were found to be naturally derived. ${ }^{5}$ Naturally derived compounds include those that are natural products, derivatives of natural products, synthetic compounds based on a natural product pharmacophore or synthetic compounds that show competitive inhibition of a natural product substrate. Natural products are not often used as drugs in their directly isolated form, they often serve as lead compounds 
resulting in the development of analogues with optimised pharmacological properties. ${ }^{5}$ Natural products chemistry can, therefore, be considered the first step in the journey to finding new, naturally-derived drugs for clinical use.

\subsection{Marine Natural Products}

The marine environment provides a rich source of interesting and previously unknown compounds, which is why it is so extensively explored in the search for new drugs. ${ }^{2,4,6}$ To date, most new compounds with interesting biological activities that have been found from marine organisms have been extracted and isolated from marine sponges, corals and other invertebrates. ${ }^{4}$ In 2010, 1003 new compounds from marine organisms were reported in the literature and $28 \%$ of these were from sponges. ${ }^{7}$ In 2011,1152 new compounds were reported from marine organisms and, of these, $26 \%$ were from sponges. ${ }^{8}$ It should also be noted that the bacteria and fungi that live in association with marine sponges, algae and corals have also been found to produce potent bioactive compounds. These microorganisms can often contribute to a significant amount of the observed biomass of their marine invertebrate host. This can result in compounds isolated from a marine invertebrate extract actually having been produced by the microorganisms associated with that invertebrate. ${ }^{2,4,9,10}$

Only a handful of drugs derived from marine natural products have been approved for clinical use today. ${ }^{11}$ By 2010, despite the 1003 new compounds that were reported in the literature for that year alone, ${ }^{7}$ only four drugs derived from marine organisms had been approved for clinical use by the United States Food and Drug Administration (FDA) and one drug had been approved by the European Medicines Agency (EMEA). ${ }^{11}$

Ziconotide (3, Prialt ${ }^{\circledR}$ ) was the first marine-derived drug that was approved by the FDA in December 2004, and by the EMEA in February 2005. ${ }^{11,12}$ Ziconotide joined the naturally-derived synthetic drugs cytarabine (4, Ara-C, Cytosar- $\mathrm{U}^{\circledR}$, Depocyt $\left.{ }^{\circledR}\right)$ and vidarabine (5, Ara-A, Vira-A ${ }^{\circledR}$ ), which were approved by the FDA in 1969 and 1976, respectively. ${ }^{2,4,11}$ Ecteinascidin-743 (6, trabectedin, ET-743, Yondelis ${ }^{\circledR}$ ) was registered with the European Union in October 2007 and the drug eribulin mesylate (7, Halaven ${ }^{\circledR}$ ) was approved by the FDA in November $2010 .{ }^{2,4,13}$ Ziconotide is the synthetic equivalent of the peptide $\omega$-conotoxin MVIIA. It contains 25-amino acids and was isolated from the venom of the fish-hunting marine cone snail Conus magus. Ziconotide is used as an analgesic, specifically for management of chronic or severe pain. ${ }^{2,11}$ Cytarabine and vidarabine are synthetic pyrimidine nucleosides that are derivatives of nucleosides isolated from the Carribean sponge Tethya crypta. Cytarabine is used in the treatment of leukemia, while vidarabine was used as an antiviral agent but was discontinued in June 2001. ${ }^{11}$ Ecteinascidin-743 is a tetrahydroisoquinoline alkaloid containing three 
fused tetrahydroisoquinoline rings. It was isolated from the tropical ascidian (sea squirt) Ecteinascidia turbinata. Ecteinascidin-743 is used to treat refractory soft tissue sarcomas and ovarian carcinoma. ${ }^{2,11}$ Eribulin mesylate is a simplified ketone analogue of the macrocyclic halichondrin B, which is a potent cell growth inhibitor that has been isolated from several unrelated sponges. Eribulin mesylate is the methansulfonate salt of eribulin (7) and is now used in the treatment of late-stage metastatic breast cancer. ${ }^{2,13,14}$

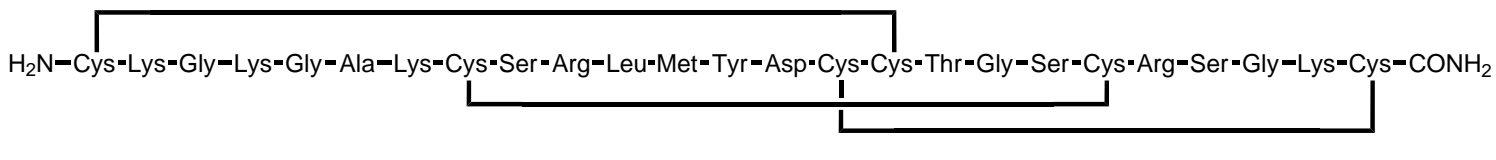

3<smiles>Nc1ccn(C2O[C@H](CO)[C@@H](O)C2O)c(=O)n1</smiles><smiles>Nc1ncnc2c1ncn2C1O[C@H](CO)[C@@H](O)C1O</smiles>

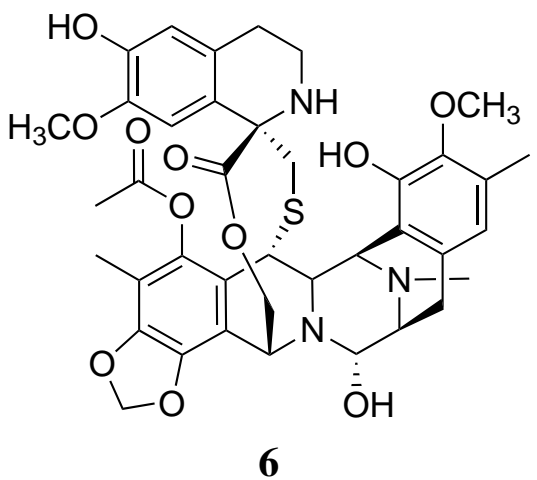

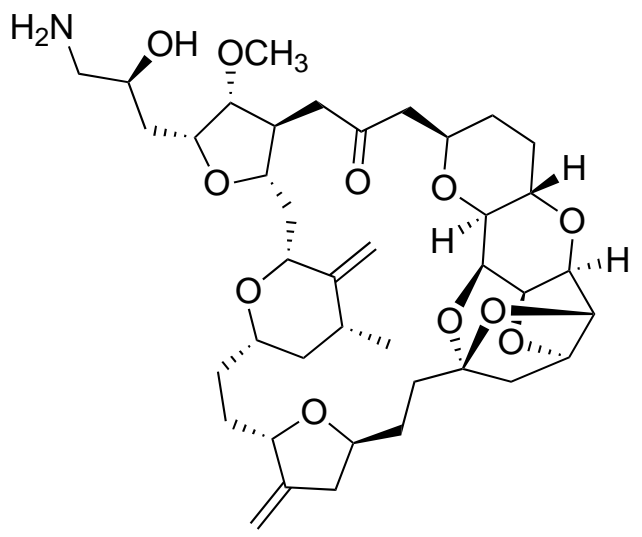

7

Of particular interest in the search for biologically active molecules are secondary metabolites. It is thought that secondary metabolites must provide some ecological advantages to the organism producing them as they are often very structurally complex and an organism would not waste the energy to produce these compounds if it was not an advantage to them. ${ }^{15}$ Secondary metabolites were initially thought to be waste products of the organisms producing them, but it was later shown that these compounds were developed as a means of defence, communication and predation. ${ }^{5}$ For sessile marine invertebrates, the action of these secondary metabolites is often thought to be defensive due to the lack of physical defences in these organisms. ${ }^{5}$ As a result of this, marine invertebrates have been found to be a rich source of secondary metabolites with unusual chemistry. ${ }^{6,15,16}$ These secondary metabolites can have activities in the organism to deter or paralyse predators, slow or suppress the growth of competing organisms, prevent 
bacterial growth on their surface (fouling) and shield from ultraviolet radiation. ${ }^{5,15,16}$ These metabolite activities are particularly useful due to the sessile nature of most of these marine invertebrates, which feed by filtration of seawater. ${ }^{10}$ Biologically active compounds isolated from marine invertebrates are often found to be very potent. This potency is necessary for these organisms because of the rapid dilution that occurs when a compound is released into the ocean. As a result, if the compounds are going to have a biological effect, they will have to be very potent. ${ }^{10,16}$

A significant problem with the bioactive compounds isolated from marine organisms is that there is often difficulty in isolating enough of the compound for complete biological testing; this is due to the natural products only being produced in trace quantities. ${ }^{4,5,10}$ As little as 40 to 50 years ago the lack of suitable spectroscopic and chromatographic techniques may have prevented the isolation and structural elucidation of compounds present in trace amounts. ${ }^{10,17}$ This difficulty in acquiring enough mass can lead to few marine natural products actually entering clinical trials and so can be considered a contributing factor to the low number of marine natural products that have actually been approved for use as drugs. ${ }^{4,10}$ However, in the last 50 years, the development and improvement of nuclear magnetic resonance (NMR) spectroscopy hardware and software has led to a greatly improved ability to solve the structure of new compounds. Further developments over the past 30 years have allowed the mass required to collect useful NMR data from a moderately sized molecule ( $\leq 500 \mathrm{Da}$ ) to fall from 20-50 $\mathrm{mg}$ to less than $1 \mathrm{mg} .{ }^{17}$ This has greatly increased the ability of natural products chemists to identify new biologically active compounds, resulting in thousands of new compounds being reported every year.

In order for comprehensive biological tests to be performed on isolated marine natural products it is often important to develop successful chemical syntheses. This can allow the biological activity of these, often very structurally complex, compounds to be fully characterised, thus allowing their potential for use as drugs to be assessed. Most of the compounds that are currently undergoing clinical trials are being produced by total synthesis as this is often the easiest and most reliable way to produce these compounds. ${ }^{4}$ Other methods used to overcome the issue of low mass availability are: aquaculture (also known as mariculture, the farming of marine organisms), semi-synthesis (modification of natural compounds, often isolated from bacteria) and synthesis of analogues. ${ }^{2}$

\subsubsection{Marine Sponges}

Sponges are sessile metazoans (multicellular organisms) of the phylum Porifera. They have existed for millions of years. Evidence of their existence dates back to the Precambrian period and they are thought to have been well established by the Cambrian period. ${ }^{18}$ They are the most ancient multicellular organisms on the planet. There are an 
estimated 15,000 sponge species living in marine and freshwater habitats worldwide, but this is thought to only be a small fraction of all the species of sponges that have ever lived. ${ }^{18}$ There do not appear to have been any significant changes in sponge morphology over the last 509 million years in which they have existed, in fact, some sponges alive today appear to be almost identical to their ancient counterparts. Consequently, sponges are regarded to be one of the most successful life forms to have ever lived. ${ }^{18}$

Classification of sponges can be very difficult, mainly due to the physical characteristics of the sponge being highly influenced by environmental factors. ${ }^{18}$ This can lead to sponges of the same species having very different colours, shapes and sizes. Sponges feed by filtering sea water through channels from external pores using choanocytes (flagellated cells) that create a unidirectional water current through the sponge. These choanocytes are unique to sponges and, along with various other cells, they remove food particles and oxygen from the water. ${ }^{18}$ This simple nature of sponges means that they do not have complex digestive, circulatory or neurological systems seen in other animals, adding to the difficulty in classifying them. Sponges are made up of highly mobile totipotent cells, which give them the ability to adapt to a diverse range of environments (plasticity) and contribute to the many different morphologies of sponges that have been observed. ${ }^{18}$ The firmness of a sponge's structure is made up of collagen fibrils (located in the mesohyl; the gelatinous matrix of the sponge), spongin fibres and silica $\left(\mathrm{SiO}_{2}\right)$ or calcium carbonate $\left(\mathrm{CaCO}_{3}\right)$ spicules, which form an inorganic skeleton for many sponge species. ${ }^{18}$ It is these spicules that form the basis for sponge classification.

There are three distinct classes of sponges: Hexactinellida, Demospongiae and Calcarea. Within these there are currently seven subclasses, 25 orders, 127 families and 682 genera. ${ }^{18}$ Demospongiae sponges are the most common sponges (about $85 \%$ of living sponges) and they are usually characterised by siliceous monaxone or tetraxone spicules and/or spongin fibres. Hexactinellida sponges are characterised by siliceous triaxone and/or hexactine spicules while Calcarea sponges are characterised by calcium carbonate spicules, which are usually triactine or tetractine. ${ }^{18}$ Silica spicules can be revealed by dissolving the organic matter of a small piece of sponge in concentrated nitric acid $\left(\mathrm{HNO}_{3}\right)$ while calcium carbonate spicules can be found by dissolving the organic matter of a small piece of sponge in bleach $(\mathrm{NaOCl})$. There are further features of sponges that can be used to identify the separate classes, these features can be used, for example, to determine the classes of sponges without spicules or to identify the order or family of a sponge within a class. The details of the full classification system is beyond the scope of this research and so will not be discussed here. For a comprehensive review on the taxonomic classification of sponges see Systema Porifera. A Guide to the Classification of Sponges. ${ }^{18}$ 


\subsection{Natural Products Screening and Isolation}

The great range and diversity of marine organisms makes it hard to decide where to start in the search for bioactive marine natural products. It is for this reason that screening techniques have been developed to identify organisms containing compounds of interest. The two main screening approaches utilised by marine natural products chemists to identify interesting compounds are bioassay and spectroscopic screens. Both of these methods use the crude extraction of a marine organism that is then assessed for interesting chemical functionality or biological activity. The use of bioassays to screen extracts means that any compounds identified will have biological activity. However, a limitation of the use of bioassays is that they specifically target one receptor or organism meaning that compounds that may have activity in other biological systems will not be identified. Another limitation of this approach is that compounds that are already known to show biological activity cannot be distinguished from novel biologically active compounds leading to re-isolation of already known compounds. Spectroscopic screens of crude extracts look to identify interesting functional groups or masses and fragment masses (for NMR and MS screens, respectively). An advantage of this technique is that known compounds can be identified a lot earlier in the isolation procedure. However, a disadvantage of this technique is that the compounds isolated using this method will not necessarily be biologically active.

Bioassay-guided isolation of marine natural products is done by screening a library of extracts to identify organisms containing compounds that are biologically active. Once these have been identified, purification of the bioactive compound from all the other compounds present in the initial extract is required. This is typically done using chromatographic techniques, with tests for bioactivity performed at each stage during purification to ensure that the desired bioactive compound is being isolated. Spectroscopic techniques can then be used to determine the chemical structure of these isolated bioactive compounds. ${ }^{16}$ Spectroscopic-guided isolation is very similar to the above methods, with the obvious difference of the spectroscopy of choice being used for the initial screen and after each stage of purification to ensure the compound of interest is being isolated. The research described herein will use a combination of bioassay and NMR-guided isolation to identify bioactive compounds and identify any other compounds of interest found during purification of the initial extracts. The assays in this work will target antifungal compounds and compounds that interact with the large mechanosensitive channels of bacteria. 


\subsubsection{Antifungals}

Fungi are eukaryotic organisms and, as such, they have metabolisms very similar to that of mammals. This means that there are few clinically viable antifungal agents due to the severe side effects that can occur with these compounds. ${ }^{1}$ Amphotericin B (8) is a well known antifungal agent but it has severe side-effects such as chills, fever and nephrotoxicity. ${ }^{1,19,20}$ Although many compounds with antifungal activity in vitro have been reported, when tested in animal models the adsorption, distribution, metabolism and excretion of these compounds have indicated that they are not suitable for use as antifungal agents for the treatment of humans without further chemical development. ${ }^{1}$

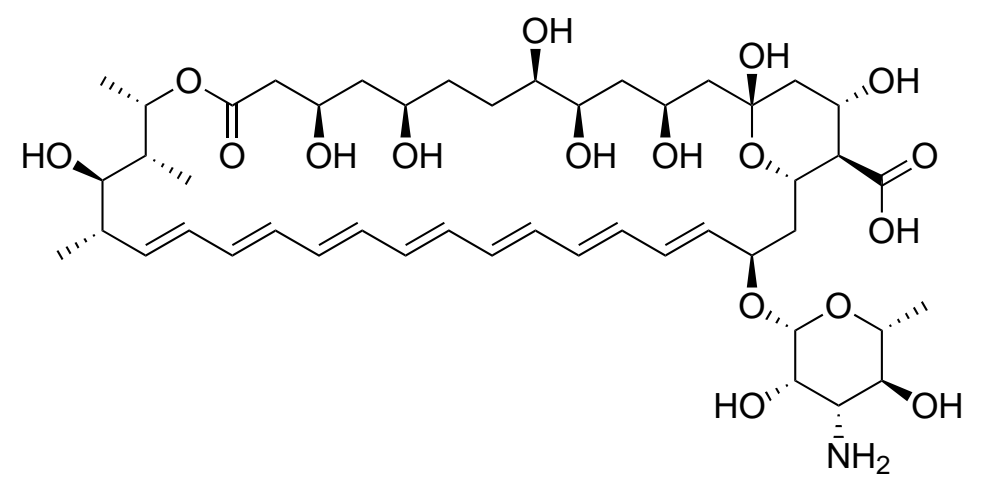

8

Generally, invasive fungal infections are not a major threat to healthy individuals, as their natural immune response is sufficient to defeat these pathogens. ${ }^{21}$ However, for individuals with compromised immunity, often due to disease (e.g. AIDS) or chemotherapy, fungal infections from these opportunistic pathogens can be a dangerous complication. ${ }^{22,23}$ For this reason, effective antifungal agents with minimal side effects are extremely desirable due to the already fragile health of these patients. ${ }^{23}$ Some potentially pathogenic fungi (also called mycoses) can be found in soil as saprophytes, where they will compete for nutrients and need to adapt quickly to a rapidly changing environment, for example Aspergillus fumigatus. ${ }^{21,24}$ However, one of the most common and successful human fungal pathogens, Candida albicans, is rarely found free-living without a mammalian host. ${ }^{25}$ C. albicans is a common fungus that can commensally live in various areas of the human body, it is also an opportunistic pathogen that will respond to changes in a hosts physiology (affecting their immune system or microflora) resulting in a fungal infection. ${ }^{25,26}$ There are a limited number of antifungal drugs active against C. albicans. These drugs also have significant limitations including the emergence of drug-resistant strains and severe side-effects. This highlights the need for new antifungal drugs to be developed. ${ }^{27}$

Fungal infections in plants are also a huge concern on a worldwide scale. For many years plant diseases caused by fungi or fungal-like oomycetes have caused devastating losses of crops worth millions of dollars and this has had a significant impact on human history. ${ }^{24,28,29}$ For example, the Irish potato famine from 1845 to 1851 was due to potato 
blight, which is caused by the fungus-like oomycete Phytophthora infestans. This caused starvation and economic ruin in Ireland. ${ }^{24,28}$ Potato blight is still considered to be the worlds most dangerous potato disease. Fungicides are available to treat potato blight but these can be costly and harmful to human health. ${ }^{28}$ Another fungal plant pathogen that is estimated to cost around $\$ 66$ billion in crops is Magnaporthe oryzae, which causes rice blast. This fungus is found worldwide and has the capability to destroy up to $100 \%$ of crops in some rice paddies. M. oryzae is particularly difficult to control as resistant rice cultivars do not display resistance to all strains of this fungus and, generally, the fungus will overcome the resistance in two to three growing seasons. Fungicides, however, are able to kill $M$. oryzae, once again showing their importance. ${ }^{28}$ Synthetic compounds currently dominate the fungicide market and issues that have arisen from their repeated use include disruption to natural biological systems caused by their environmental pollution, the development of fungicidal resistance and the residual toxicity of these fungicides resulting in human health concerns. ${ }^{29}$ There is now a movement to develop more biopesticides, such as natural product fungicides, which cause less environmental disruption and are safer for humans so that the synthetic pesticides can be phased out. ${ }^{29}$

Fungal infections in animals have also proven to be an increasing concern. For example, the emergence of white-nose syndrome (WNS) in bats, first documented in the United States (US) in New York State in February 2006, has led to fears of regional extinction of the little brown bat, which was previously a common bat species in the US. ${ }^{30,31} \mathrm{WNS}$ is a psychrophilic fungal infection caused by the fungus Geomyces destructans that affects hibernating bats. The disease is named for the white fungal growth observed on the muzzle, ears and wing membranes of affected bats. ${ }^{30,32}$ One of the effects of this fungus on the bats is a loss of fat reserves, which are crucial for a successful hibernation. ${ }^{30}$ It is also thought that this disease has effects on the bats' behaviour. ${ }^{31,33}$ WNS now occurs in northeastern and mid-Atlantic regions in the US as well as the provinces of Ontario and Québec in Canada. ${ }^{31}$ It is fast spreading and can cause drops in bat populations ranging from 30 to $99 \%$ per year once a hibernaculum is infected. The regional mean decrease in bat population for infected hibernacula is $73 \%$ per year and hibernacula are generally infected within 2 years of WNS being observed in that region. ${ }^{31}$ WNS is known to affect at least seven species of hibernating bats. ${ }^{31}$ It has been predicted that there is a $99 \%$ chance of regional extinction of the little brown bats in the next 13 years if the trends observed in 2010 for WNS continue. ${ }^{31}$ Even if circumstances such as WNS mortality reduces over time, the regional population of little brown bats is expected to drop to $1 \%$ of its size prior to the emergence of WNS in less that 20 years time. ${ }^{31}$ This decline in the insectivorous bat population is expected to have significant detrimental effects on the ecology and economy of these regions in coming years. ${ }^{30,33}$ The development of an antifungal treatment for WNS could save the regional little brown bat populations and reduce the detrimental ecological and economic effects in affected regions. 


\subsubsection{Antibacterials}

The discovery of penicillin (9) in 1928 did not gather much interest until its rediscovery as an antibiotic in the early 1940 s by scientists at Oxford University. ${ }^{34}$ Since this rediscovery, antibiotics have been used to treat infectious diseases and, in particular, many natural product antibacterials have been discovered and used to treat bacterial infections. ${ }^{3,35}$ However, a major problem that has developed with the widespread use of antibacterials is the emergence of antibacterial-resistant bacterial strains. Within five years of the first use of penicillin, $50 \%$ of isolated Staphylococcus aureus exhibited resistance. ${ }^{34}$ Bacteria and fungi have very rapid reproduction rates and so they can mutate and evolve quickly, adapting to new threats. The widespread use of antibacterials was thus able to trigger the evolution of antibacterial resistant strains of bacteria. ${ }^{3,35}$ New classes of antibacterials are being sought to combat the drug-resistant strains of bacteria by affecting different targets. Synthetic tailoring of known antibacterial scaffolds has been used in the past to develop new antibacterial drugs but these are only short term solutions in treating multidrug resistant bacterial infections as resistance inevitably develops. ${ }^{34,36,37}$

A particularly concerning bacteria that has developed resistance to current drug treatments is methicillin-resistant Staphylococcus aureus (MRSA). ${ }^{3,38}$ Methicillin (10), an analogue of penicillin, was developed in 1960 in order to combat the penicillin resistant strains of $S$. aureus, which were becoming more common. ${ }^{3,34}$ However, resistance to methicillin quickly developed in S. aureus and by the 1970s and late 1980s MRSA had been identified widely in Europe and the US, respectively. ${ }^{34}$ Between 1999 and 2005, the estimated number of $S$. aureus-related infections resulting in hospitalisation in the US increased by $62 \%$ and the number of estimated MRSA hospitalisations increased by $119 \% .{ }^{38}$ Patients with MRSA bacteremia have a higher risk of mortality than patients with methicillinsusceptible $S$. aureus bacteremia. However, whether this increased risk of mortality is due to enhanced virulence of MRSA, poor efficacy of drugs used for treatment, delayed MRSA-appropriate treatment or another as yet unidentified reason has not been determined. ${ }^{39}$

MRSA is a nosocomial (acquired in hospital) infection due to the frequent use of antibiotics in that environment. ${ }^{3}$ MRSA does not just exhibit resistance to methicillin but it is resistant to all of the $\beta$-lactam antibiotics that are otherwise used to treat infections caused by $S$. aureus such as penicillin, oxacillin and amoxicillin. ${ }^{40-43}$ While MRSA is most commonly acquired in hospitals, community-acquired MRSA is also becoming more common. ${ }^{44,45}$ Community-acquired MRSA is defined as MRSA contracted by a patient who has not had recent contact with the healthcare system. Usually, communityacquired MRSA presents itself as a skin infection and can be treated without longterm effects on the patient. In contrast, nosocomial invasive MRSA infections are more severe and can be life-threatening. ${ }^{40,41,44,46}$ Vancomycin (11) is an antibacterial that was previously used as a last resort against MRSA but is now more commonly used 
in its treatment. Unfortunately, even resistance to vancomycin has started to emerge highlighting the need for new classes of antibacterials to be developed. ${ }^{3,41,45,47}$ Antibiotic resistance in the bacteria Streptococcus pneumoniae and Mycobacterium tuberculosis, which are common respiratory pathogens, are also causing great concern in the medical community. The growing resistance of these and other bacterial pathogens have resulted in a critical need for the discovery of new antibacterial agents with novel modes of action. ${ }^{36,48}$<smiles>CC1(C)S[C@@H]2[C@H](NC(=O)Cc3ccccc3)C(=O)N2[C@H]1C(=O)O</smiles>

9<smiles>COc1cccc(OC)c1C(=O)N[C@H]1C(=O)N2[C@@H](C(=O)O)C(C)(C)S[C@H]12</smiles>

10

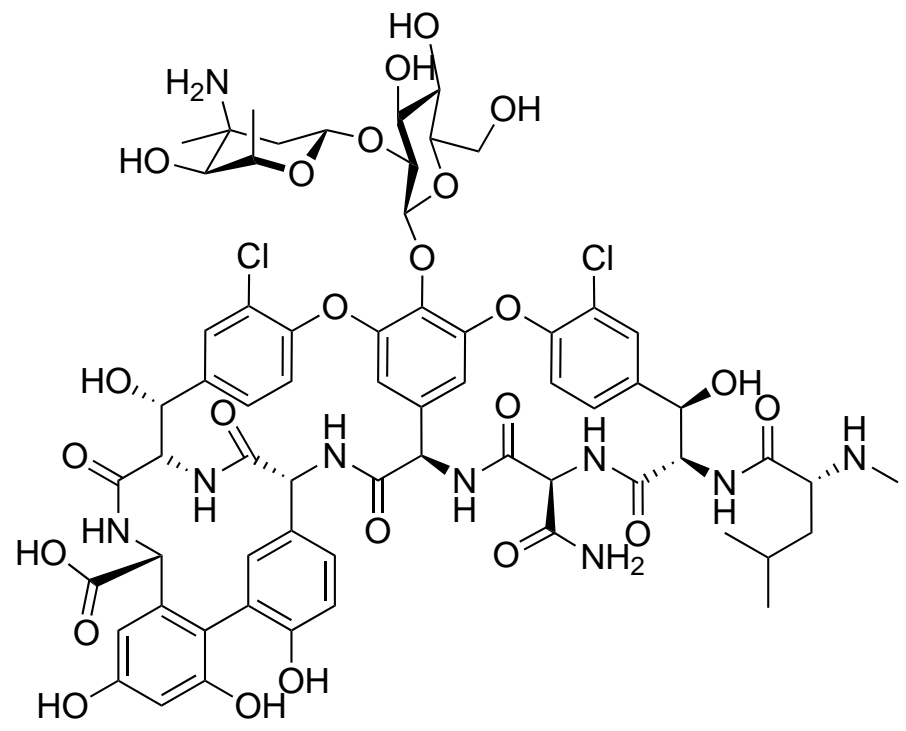

11

\subsection{Research Aims}

The aim of the current research was to isolate and identify bioactive compounds from marine invertebrates. The marine invertebrates being studied were a range of New Zealand and Tongan sponges, algae and tunicates of which a library of extracts had been made. In particular, compounds that have antifungal or antibacterial activity were to be studied. This involved using an assay to test the ability of extracts to inhibit the growth of the yeast Saccharomyces cerevisiae to find antifungal compounds. To find antibacterial compounds, a new assay targeting the opening of large mechanosensitive channels (MscL) of Escherichia coli was further developed and tested against extracts. 
By screening extracts of marine invertebrates for antimicrobial activity, a chemically diverse range of compounds are able to be tested. This results in a chance for completely new structural classes of antimicrobial drugs to be discovered. New structural classes being developed for antimicrobial drugs is advantageous over synthesising analogues of existing drugs as microbes that are already resistant to a drug are likely to have some resistance to its analogues as well. It is therefore likely that new structural classes of antimicrobials will have more success in clinical development. ${ }^{34-37}$

The MscL assay was modified to be performed at VUW to screen the invertebrate extract library for extracts with molecules capable of locking MscL in an open conformation. The $S$. cerevisiae assay was used to identify invertebrate extracts with molecules that were cytotoxic or cytostatic towards the growth of yeast. Once active extracts were identified from the $S$. cerevisiae assay, extractions were repeated in bulk and the active compounds isolated using chromatographic techniques. The isolation of the bioactive compounds was guided using a combination of bioassays and ${ }^{1} \mathrm{H}$ NMR spectroscopy. The structures of the isolated bioactive compounds were then identified using 1D and 2D NMR spectroscopy and mass spectrometry (MS). The activity of these isolated compounds was finally tested in assays against the clinically relevant fungi Candida albicans. 


\section{Chapter 2}

\section{Bacterial Mechanosensitive Channels}

\subsection{Discovery of Mechanosensitive Channels}

Bacterial cells have an optimal turgor pressure (pressure of the cytoplasm on the cell membrane, holding it against the cell wall), which they work to keep constant despite changes in the osmolarity of their environment. Different bacteria have different optimal turgor pressures. Bacteria grown in a high osmolarity (high solute concentration) environment maintain their turgor pressure by accumulating solutes (such as potassium ions, glutamate and proline) in the cytosol, so that water will not diffuse out into the environment. Cells will also accumulate solutes when transferred from a low osmolarity environment to a high osmolarity environment to counteract the water leaving the cell as a result of this osmolarity change (hyperosmotic shock). ${ }^{49,50}$ When these cells are then transferred to lower osmolarity environments, water will rapidly diffuse into these cells that have a higher osmolarity; this is known as hypoosmotic shock. This can increase the turgor pressure in the cell by approximately 10 atmospheres in a few milliseconds. The increase in turgor pressure will increase the membrane tension and can cause the cell to burst if there is no release of pressure (Figure 2.1). ${ }^{49,50}$

Mechanosensitive channels (Msc) are channels located in the cytoplasmic cell membrane that open in response to mechanical forces acting on the cell membrane; this includes stretch-activated and stretch-inactivated channels. ${ }^{51-53}$ Msc have been found in bacteria, fungi, plants and animals. While these channels all have mechanosensitive functions, it is possible that their protein sequences and therefore mechanism of mechanosensitivity could be unrelated. ${ }^{54,55}$ The most studied Msc in bacteria are poorly selective channels that are stretch-activated. The mechanical force acting on the bacterial cell membrane is typically an increase in membrane tension (stretch) caused by osmotic swelling as a result of hypoosmotic shock. ${ }^{51-53,55}$ In response to this, Msc will open and passively release solutes from the cell until the osmolarity of the cell and media are equal, the Msc will 
then close and allow normal growth to resume in the cell (Figure 2.1). ${ }^{50,56}$ Modifications to mechanosensitive processes to sense external mechanical stimuli may have led to the evolution of many sensory functions that are currently used by different organisms, such as touch or hearing. ${ }^{57}$ It is hoped that a better understanding of bacterial Msc can give some insights into the poorly understood molecular basis of such senses. ${ }^{56}$

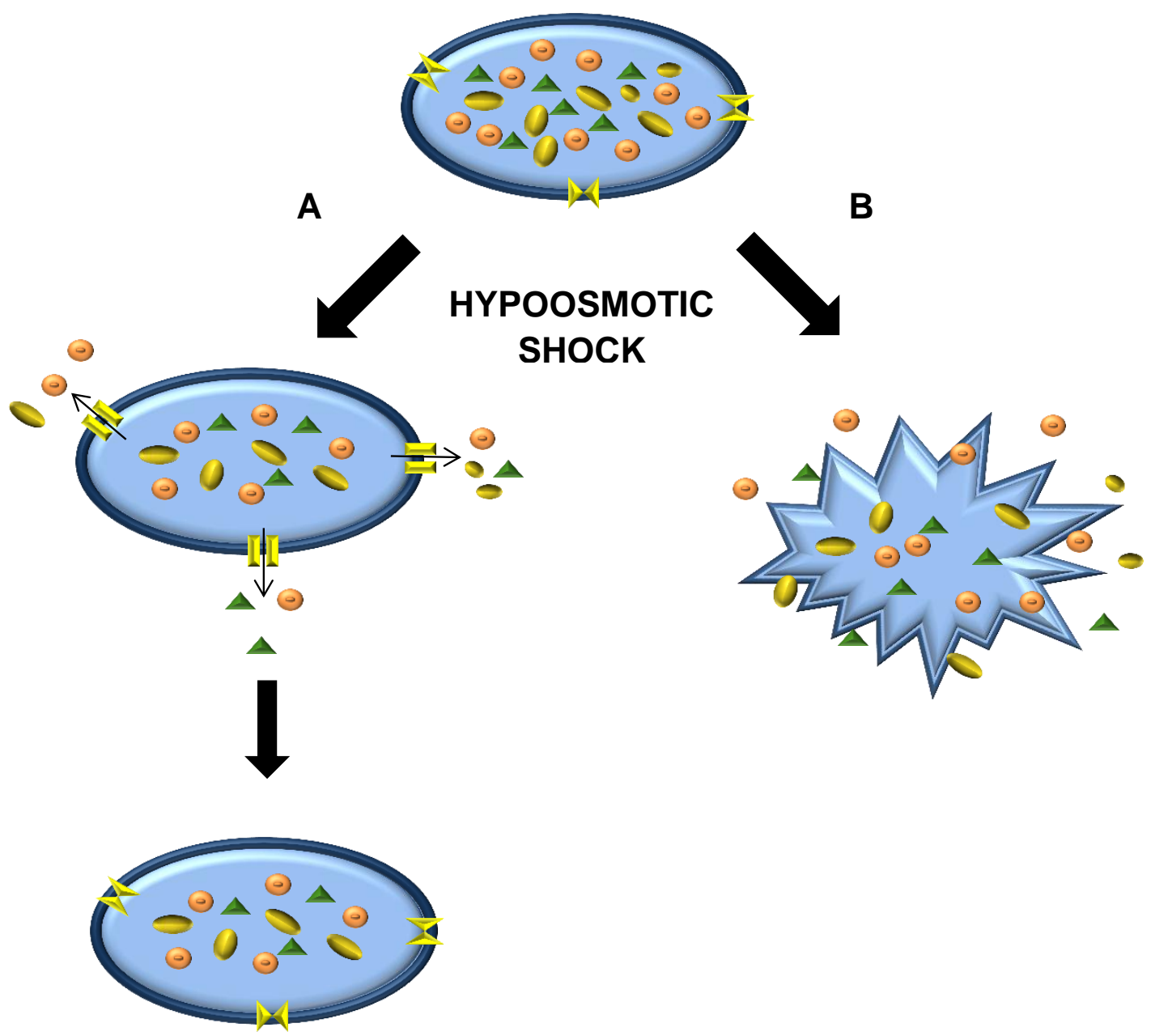

Figure 2.1. Msc in osmotic regulation. When a cell in osmotic balance is moved to a lower osmolarity media than its internal osmolarity, hypoosmotic shock will occur and the cell will begin to accumulate water increasing the turgor pressure. At this point either (A) Msc will sense the increase in membrane tension caused by the accumulation of water and open, releasing solutes into the media and allowing normal cell growth to occur once an ideal turgor pressure is reached, or (B) if Msc do not open or no Msc are present, the cell will continue to accumulate water, increasing the turgor pressure until the cell eventually lyses. This figure is adapted from Booth et al. (2007). ${ }^{50}$

Berrier et al. found that when inducing hypoosmotic shock in Escherichia coli cells, the amount of internal ATP, lactose, glutamate and potassium ions reduced. By altering the concentration of solute $(\mathrm{NaCl})$ in the solution that the cells were transferred to (to cause hypoosmotic shock) they were able to show that the extent of internal solute loss depended on the degree of hypoosmotic shock. Also, reducing the temperature of the solution increased the amount of efflux occurring as well. The $90 \%$ viability found when 
culturing the hypoosmotic stress-induced cells was evidence that the release of internal solutes was not due to complete cell lysis and hence could be due to the presence of Msc. Further evidence for Msc was that the internal solutes travelled out of the cells very fast, indicating that the route by which efflux occurred was not that of conventional transport systems. ${ }^{53}$ Studies conducted by Levina et al. on the transfer of E. coli from high osmolarity to low osmolarity media have shown that the threshold at which Msc are significantly activated is when a hypoosmotic shock from a difference of more than $150 \mathrm{mM}$ of $\mathrm{NaCl}$ occurs. This was found by transferring E. coli cells that had been grown in media with a set concentration of $300 \mathrm{mM} \mathrm{NaCl}$ to media with lower concentrations of $\mathrm{NaCl}$ that had been acidified so that cell death would occur upon opening of the poorly selective Msc. ${ }^{49}$

\subsection{Techniques for Measuring Msc}

A common technique used to study Msc is the patch-clamp technique; this has provided a lot of information to further understand Msc. ${ }^{58}$ The patch-clamp technique allows the current through individual ion channels to be recorded by using a recording patch-pipette to suck a section of the cell membrane of an enlarged cell into the tip, forming a high resistance "gigaohm" seal. Pressure and a voltage can then be applied to this 'patch' of the membrane and measurement of the current amplitude of the Msc at a known pressure and membrane voltage ('holding potential') can then be recorded. ${ }^{50,52,54-56,59,60}$ Negative pressure (suction) in the pipette is required to open these Msc as it pinches a section (patch) of the cell membrane increasing the membrane tension in that section, thus causing the Msc to open. ${ }^{54}$ It has been found that larger diameter pipettes require lower pressures (greater suction) to activate the Msc, indicating that it is in fact the tension and not the pressure that is activating these channels. ${ }^{61}$ The channels with greater conductance have been found to require greater suction to be opened. The actual pressure required to open the channels, however, is not as important as the relative magnitudes due to the variations that occur from patch to patch (e.g. different radii of curvature) ${ }^{52}$ In general, the strength of suction required to open the Msc of large conductance (MscL) is about 1.5 times the magnitude of the suction required to open the Msc of small conductance (MscS). ${ }^{62}$ For representative images of a patch-clamp and a trace of the current and pressure information extracted from a patch-clamp experiment see Figure 2 of Booth et al. (2007). ${ }^{50}$

Mechanosensitive channel activity can be measured from reconstituted proteoliposomes and giant bacterial spheroplasts. The reason for using giant spheroplasts is that the patch pipette opening is too large for the patch-clamp technique to be used on normal bacterial cells, therefore larger objects need to be used. ${ }^{52,54,59}$ Proteoliposomes are made from liposomes fused or reconstituted with purified membrane fractions of bacteria (often

E. coli) or purified Msc proteins. ${ }^{52,59,62,63}$ The observation that single patches in these 
proteoliposomes often have several channels with a single type of conductance has led to the hypothesis that these membrane channels separate in the reconstitution procedure and then form clusters of one type of channel in the formation of the proteoliposomes. ${ }^{52}$

Giant spheroplasts can be formed in different ways. One way of forming these spheroplasts involves growing cells in the presence of chemicals such as mecillinam (6-amidinopenicillanic acid) that prevent peptidoglycan synthesis, inhibiting the formation of the cell wall. ${ }^{54,63,64}$ Giant spheroplasts can also be formed by growing cells in the presence of cephalexin, which prevents cell division. These long, thin (filamentous) cells can then be treated with lysozyme and EDTA (ethylenediaminetetraacetic acid), which will dissolve the cell wall. ${ }^{52,54,59}$ Bacteria with mutations to give a round phenotype can also be used to give giant round spheroplasts. ${ }^{52}$ It should be noted that when patch-clamp experiments were performed on E. coli membrane vesicles fused with azolectin liposomes, the pressure required to open the Msc was lower than the pressure used to open the Msc in E. coli giant spheroplasts. ${ }^{59}$ Mechanosensitive channels that have been reconstituted into proteoliposomes have been found to remain closed in the absence of suction (membrane tension) regardless of the voltage applied to the membrane. When suction is applied causing the channels to open, once the suction (and therefore tension) is released, the channels will close again. ${ }^{52}$

\subsection{Distinction of Mechanosensitive Channels}

In bacteria there are two different subfamilies of Msc-the mechanosensitive channel of large conductance (MscL) subfamily and the mechanosensitive channel of small conductance (MscS) subfamily. In E. coli, only one homologue of MscL and at least six MscS homologues exist: the canonical MscS, the subsequently identified MscK (potassium-dependant mechanosensitive channel; originally KefA), the YbdG channel (previously thought to be the mechanosensitive channel of mini conductance, MscM) and a further three MscS homologues, which have recently been characterised by electrophysiology and functional analyses (YjeP, YbiO and YnaI). Where the channel YjeP is now proposed to be the major component of the MscM channel activity that has been observed. ${ }^{56,65} \mathrm{MscL}$ and $\mathrm{MscS}$ are the most well studied of these Msc and therefore they are the best understood. The MscL and MscS are so named based on the relative conductance of the channels in the (inner) cytoplasmic membrane of E. coli as measured by Sukharev et al. using the patch-clamp technique. ${ }^{59}$ The conductance of E. coli MscL (Ec-MscL) was found to be about $3100 \mathrm{pS}$ and the conductance of E. coli $\mathrm{MscS}$ (Ec-MscS) was about $900 \mathrm{pS}$ in giant spheroplasts in a buffer solution with a $\mathrm{KCl}$ concentration of $200 \mathrm{mM} .{ }^{59}$ Higher suction pressures (and therefore higher membrane tension, which is related by Laplace's law using the radius of curvature of a membrane patch) on the cell membrane were required to open the MscL as compared with the MscS. 
In fact, the membrane tension required to open $\mathrm{MscL}$ is close to the tension at which cell lysis will occur. ${ }^{52,55,59,61}$ The presence of both MscL and MscS in E. coli could allow the cells to lose only small ions such as potassium and keep larger metabolites (whose loss would be the least favourable) in the cell when only small reductions in osmolarity of the bacterial cell environment occur. ${ }^{53}$ The number of different Msc that have been found may also be required for redundancy, due to the importance of their function in osmoregulation. ${ }^{52}$ The MscS stay open for longer durations compared with MscL, which only open for brief periods. ${ }^{52,59}$ In the study by Sukharev et al., MscL also appeared to gate at subconducting levels where the channel appeared to be partially open due to a lower conductance being observed. $\mathrm{MscS}$ also appeared to have a very slight preference for anions to pass through the channel. ${ }^{59}$

In a further study Berrier et al. identified three Msc in reconstituted giant liposomes and giant round spheroplasts that were made from E. coli cells. They identified an MscL channel that showed conductance over a range of 1000 to $2300 \mathrm{pS}$ and an MscS channel that showed conductance of 300 to $500 \mathrm{pS}$ in a $\mathrm{KCl}$ concentration of $100 \mathrm{mM}$. They also identified a previously unknown Msc conductance of 100 to $150 \mathrm{pS}$ with even slower kinetics than the MscS (it stayed open even longer). It was concluded that this was a third family of Msc and so it was named MscM for Msc of mini conductance. ${ }^{52}$ However, recent studies indicate that MscM belongs to the subfamily of MscS-like channels. ${ }^{65}$

\subsection{Large Mechanosensitive Channels (MscL)}

\subsubsection{MscL Gene}

In 1994 Sukharev et al. identified the gene responsible for producing the MscL protein in E. coli. This gene was named $m s c L$. From the sequencing of this gene it was predicted that the MscL protein had 136 amino acid residues and a molecular weight of approximately $17 \mathrm{kDa}{ }^{66}$ The MscL channel protein was, however, thought to be approximately 60 to $80 \mathrm{kDa}$ based on the elution profile of the protein when purified using size-exclusion chromatography, performed under non-denaturing conditions. ${ }^{59}$ This was thought to indicate oligomerization of the $17 \mathrm{kDa}$ protein to form the MscL. ${ }^{66}$ In this study, an $m s c L$ knock-out mutant of $E$. coli was created. The removal of the $m s c L$ gene did not inhibit viability of these bacteria, indicating that the MscL protein is not necessary to survival with transient osmotic changes. It was proposed that the reason for this is redundancy between MscL and MscS. ${ }^{66}$

After the $m s c L$ gene had been identified in E. coli, homologues of MscL were able to be identified in many other prokaryotes from unfinished sequences of genome sequencing projects and by DNA sequencing of bacteria closely related to E. coli. ${ }^{62,67}$ The UniProt 
database currently lists 2296 members of the MscL subfamily, with homologues identified in Gram-negative and Gram-positive bacteria as well as phytoplasma and mycoplasma (which are specialised cell-wall deficient bacteria). ${ }^{68-70}$ As seen in E. coli, these MscL homologues, when expressed in E. coli cells devoid of native MscL, have no effect on the activity of the native $\mathrm{MscS}$, indicating no interaction between the two proteins. These homologues also showed a requirement for pressures higher (about 1.5 times the size) than those required to activate $\mathrm{MscS}$ to be activated, as was also observed for native Ec-MscL. ${ }^{62}$ The conductances of these channels were measured to be between 3000 and $5000 \mathrm{pS}$ and they also showed poor ion selectivity. These factors seem to indicate that only minor differences exist between these channel homologues. The kinetics of these channels, however, appears to vary. The channels can be grouped into two classes, a rapid class and a slow class. The rapid class have channel open times of less than $4 \mathrm{~ms}$ to $10 \mathrm{~ms}$, and the slow class have channel open times above $10 \mathrm{~ms}$ to greater than $30 \mathrm{~ms}$. The Ec-MscL showed open times of $35 \mathrm{~ms}$ on average and so falls into the slow MscL class. $^{62}$

Conceptual translation of each of the genes studied showed similar hydropathy profiles with two hydrophobic segments (thought to be the transmembrane segments) and hydrophilic termini (both thought to lie in the cytoplasm of the cell) being found for all the proteins investigated. The carboxy-terminus showed some conservation amongst the homologues but complete divergence was also seen between the two most distantly related homologues. Also, a periplasmic loop (that connects the two transmembrane segments) appears to vary in size, with sequence similarity only being observed between the Gram-negative MscL homologues. The amino-terminus also appears to show some degree of conservation between these homologues. ${ }^{62}$

\subsubsection{Crystal Structure}

In 1998, Rees and co-workers reported the crystal structure of MscL isolated from the bacteria Mycobacterium tuberculosis (Tb-MscL), determined at $3.5 \AA$ resolution. This crystal structure was subsequently refined by Steinbacher et al. in 2007 using the diffraction data collected by Rees and co-workers. ${ }^{67,71}$ The Tb-MscL protein has 151 amino acids with a $37 \%$ sequence identity to the MscL of E. coli (Ec-MscL). The protein was found to be a homopentamer and can be divided into transmembrane, periplasmic and cytoplasmic domains. It is approximately $85 \AA$ long and, of this, about $50 \AA$ is the transmembrane domain (with about $35 \AA$ actually spanning the membrane), which is also about $50 \AA$ wide. The cytoplasmic domain is about $35 \AA$ long and $18 \AA$ wide. For each of the subunits of the Tb-MscL homopentamer, the amino- and carboxytermini were thought to be disordered in the original structure refinement in $1998 .{ }^{67}$ In the structure refinement in 2007, the first 12 residues of each subunit, at the amino-terminus, 
are modelled to be an $\alpha$-helix. It is proposed that this amino-terminal helix would sit at the membrane surface in the cytoplasm. ${ }^{71}$ The transmembrane domain is made up of two transmembrane $\alpha$-helices from each subunit, which start from the amino-terminus on the cytoplasmic face of the membrane and are joined by a loop on the periplasmic side. ${ }^{67}$ This periplasmic loop structure was also corrected in the structural refinement in $2007 .{ }^{71}$ The cytoplasmic domain is made up of an $\alpha$-helix that starts from a short loop joining it to the second transmembrane helix of the subunit and ends with the carboxy-terminus (Figure 2.2). ${ }^{67} \mathrm{~A}$ register error of six of the residues of this cytoplasmic helix was also corrected in the structural refinement of $2007 .^{71}$

The pore of this $\mathrm{Tb}-\mathrm{MscL}$ structure runs through the five-fold axis of the homopentamer. The first transmembrane (TM1) helices (that are closest to the amino-termini) of each subunit form the inner lining of the pore and the second transmembrane (TM2) helices run down the outside of these. The transmembrane helices are tilted at an angle of approximately $28^{\circ}$ to the fivefold axis and the cytoplasmic helices are tilted at an angle of approximately $15^{\circ}$ according to the original structural refinement in $1998 .{ }^{67}$ In the 2007 refinement, the transmembrane helices are described as forming a "right-handed helix bundle" whereas the cytoplasmic helices are described as forming a "left-handed" helix bundle. ${ }^{71}$ The TM1 helix of each subunit is in contact with its corresponding TM2 helix, the TM1 helices of two adjacent subunits and the TM2 helix of one of the adjacent subunits. The TM2 helix of each subunit is about $20 \AA$ from the TM2 helix of the neighbouring subunits. ${ }^{67,71}$ The amino-terminal $\alpha$-helix of a subunit is positioned in between the TM1 and TM2 helices of neighbouring subunits in an almost perpendicular orientation, with the amino-terminal pointing towards the adjacent cell membrane. ${ }^{71}$ The pore of Tb-MscL forms a funnel shape with a pore size ranging from approximately $18 \AA$ at the periplasmic face to $2 \AA$ at the cytoplasmic face. The pore is partially obstructed at the cytoplasmic face. In the transmembrane domain of this protein the inner lining of the pore is hydrophilic, except for near the cytoplasmic surface where the pore is lined with hydrophobic residues. ${ }^{67,71}$ The small (approximately $2 \AA$ ) pore size and the hydrophobic lining at the cytoplasmic end of the channel could create a seal indicating that this is crystal structure of the closed form of Tb-MscL. ${ }^{50,67}$ The open pore diameter for MscL in E. coli (Ec-MscL) has been previously approximated to be about $40 \AA \AA^{72}$

In 2009, Rees and co-workers reported the crystal structure of the MscL from Staphylococcus aureus (Sa-MscL) with a truncation of the carboxy-terminus (Sa-MscL(C $\Delta 26)$ ). This crystal structure was obtained at a resolution of $3.8 \AA$. The Sa-MscL protein has 120 amino acid residues before the truncation and this has a $40 \%$ sequence homology with Tb-MscL and a 51\% sequence homology with Ec-MscL. ${ }^{76}$ The open state of the Sa-MscL appears to be less stable than that of Ec-MscL, this is indicated by shorter open dwell times, which had been previously reported when the conductance of homologues of Ec-MscL were measured. ${ }^{62} \mathrm{Sa}-\mathrm{MscL}(\mathrm{C} \Delta 26)$ was proposed to be more stable than the full-length Sa-MscL. In patch-clamp experiments on $\mathrm{Sa}-\mathrm{MscL}(\mathrm{C} \Delta 26)$ reconstituted 


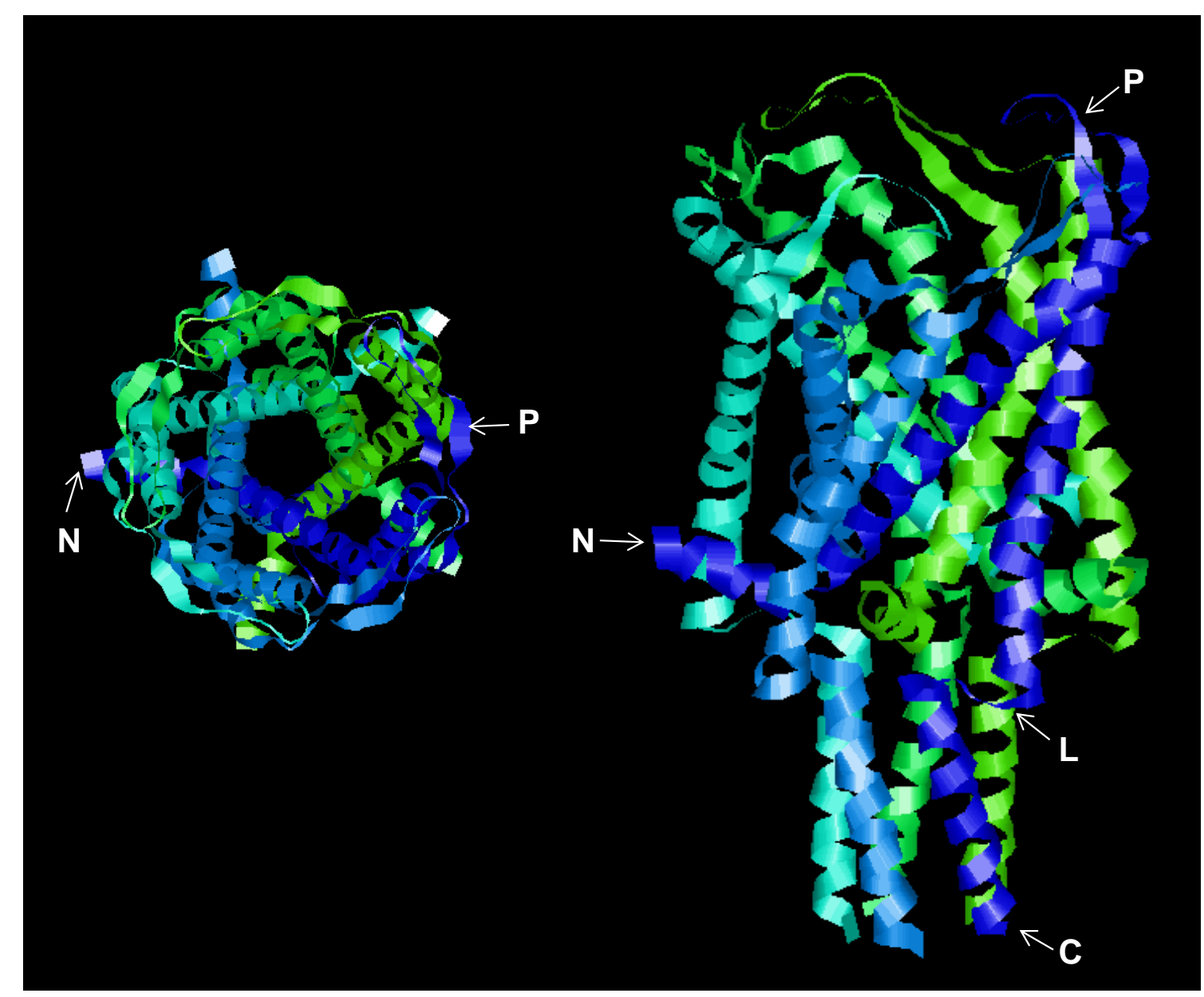

Figure 2.2. Crystal structure of the MscL protein isolated from Mycobacterium tuberculosis (Tb-MscL). It shows the homopentameric structure that is divided into a transmembrane domain and a cytoplasmic domain. For each subunit it can be seen that the transmembrane domain starts from the $\alpha$-helix amino-terminal $(\mathbf{N})$, followed by the two transmembrane helices that are joined by a periplasmic loop $(\mathbf{P})$. The cytoplasmic domain starts from a second loop $(\mathbf{L})$ that connects

the two transmembrane helices to the $\alpha$-helix of the cytoplasmic domain that ends with the carboxy-terminal $(\mathbf{C})$. The image on the left is the view through the pore axis from the periplasmic face and the image on the right is the view through the membrane. This Figure has been adapted from Chang et al. (1998). ${ }^{67}$ This structure file was downloaded from the Protein

Data Bank ${ }^{73}$ and was rendered using RasMol Molecular Renderer, version 2.7.5.2. ${ }^{74,75}$

in azolectin liposomes, the gating and conductance of these channels was found to be comparable to that of Ec-MscL. Both Sa-MscL and Sa-MscL $(\mathrm{C} \Delta 26)$ were found to be able to rescue hypoosmotically-shocked Msc-knockout $E$. coli cells, indicating that the truncated protein is still active as a MscL channel. ${ }^{76}$

The crystal structure of $\mathrm{Sa}-\mathrm{MscL}(\mathrm{C} \Delta 26)$ showed that the protein is approximately $69 \AA$ in diameter and $37 \AA$ long. Like Tb-MscL, the subunits of $\operatorname{Sa}-\operatorname{MscL}(\mathrm{C} \Delta 26)$ have two transmembrane helices (TM1 and TM2) that start from the irregularly structured carboxyterminus at the cytoplasmic face. TM1 of $\operatorname{Sa}-\operatorname{MscL}(\mathrm{C} \Delta 26)$ is joined to TM2 by a periplasmic loop as is also seen in Tb-MscL. The transmembrane domain in the crystal structure of $\mathrm{Sa}-\mathrm{MscL}(\mathrm{C} \Delta 26)$ is about $13 \AA$ shorter than the transmembrane domain of Tb-MscL and it is also up to $17 \AA$ wider. This corresponds to a wider pore being observed in the Sa-MscL $(\mathrm{C} \Delta 26)$ crystal structure. The constricted end of the funnel shaped pore, 
which is approximately $2 \AA$ wide and thought to form a seal due to the hydrophobic residues in Tb-MscL, is approximately $6 \AA$ wide in the $\operatorname{Sa}-\operatorname{MscL}(\mathrm{C} \Delta 26)$ crystal structure. The biggest difference between the Tb-MscL and $\operatorname{Sa}-\operatorname{MscL}(\mathrm{C} \Delta 26)$, which is also likely to be the reason for other discrepancies observed in the crystal structures, is that the Sa-MscL $(C \Delta 26)$ was crystallised as a homotetramer. ${ }^{76}$ More recent studies have shown that in vivo the $\mathrm{Sa}-\mathrm{MscL}$ and $\mathrm{Sa}-\mathrm{MscL}(\mathrm{C} \Delta 26)$ proteins are predominantly pentamers. It is proposed that the tetrameric structure of $\mathrm{Sa}-\mathrm{MscL}$ is not physiologically relevant but is formed as a detergent-dependent reorganisation. This tetrameric reorganisation has been found to be reversible when the detergent used to solubilize the Sa-MscL is exchanged for a more appropriate one. ${ }^{77,78}$ It is proposed that the $\mathrm{Sa}-\mathrm{MscL}(\mathrm{C} \Delta 26)$ crystal structure, which was originally thought to be in an intermediate state in the transition from a closed to open form, is actually a strained closed state that forms as a result of the tetrameric reorganisation.

\subsubsection{Mechanism}

Considerable effort has gone into elucidating the mechanisms of MscL. This is because of the proposed ability for such mechanosensitive channels to provide insight into understanding phenomena such as touch, hearing, gravitropism and other processes that require mechanosensors, by providing a simplified model system. ${ }^{71,79}$ Understanding the mechanism of these mechanosensitive channels could also provide information on the relationship between a protein and the membrane in channel gating. ${ }^{71}$ It is thought that Msc proteins are responsible for detecting the change in membrane tension without the aid of other proteins; this is due to the fact that Msc proteins are able to be purified and incorporated into liposomes and these reconstituted Msc are still able to sense membrane tension and open and close reversibly. ${ }^{61,80} \mathrm{MscL}$ and $\mathrm{MscS}$ are gated channels and, as such, the change in conductance between the open and closed state is able to occur via conformational changes of the protein. ${ }^{81}$

Since the crystal structure of Tb-MscL was proposed, more research has been directed towards elucidating the mechanism for the channel gating in Msc. It has been found that the MscL has multiple conducting states, and so is not just simply open or closed. Thermodynamic calculations have been used to show that the rate limiting step in the MscL gating process is the transition from the closed state to the lowest subconducting state. The states above this lowest subconducting state are all about the same energy and so applying tension to the membrane lowers the energy of these states equally. ${ }^{61}$ From the crystal structure of $\mathrm{Tb}-\mathrm{MscL}$ it is expected that the pore size will have to undergo a drastic change in size (both reducing the pore length and increasing the width) in order to have an approximately $3000 \mathrm{pS}$ conductance. It is thought the pore size will have to increase by approximately $600 \AA^{2}$ from the closed state in order to accommodate the observed 
conductance (forming an open pore diameter of 30 to $40 \AA$ ). ${ }^{61}$

Martinac and co-workers used electron paramagnetic resonance (EPR) spectroscopy and site-directed spin labelling (SDSL) to identify the structural changes involved in the opening of MscL. From the results obtained using this technique, they proposed that the crystal structure of Tb-MscL obtained by Rees and co-workers was a good representation of the active protein in a membrane environment. ${ }^{67,80}$ One of the problems that had to be overcome to use this method in identifying the changes in the protein structure was how to open the MscL without using pressure. ${ }^{80}$ Using phosphatidylcholine (PC) liposomes to reconstitute the MscL protein and performing patch-clamp analyses on these, Martinac and co-workers found that thinner bilayers (with monosaturated chains of 16 carbons, PC16) required less pipette pressure to open MscL than the control (with 18-carbon chains, PC18) and more pipette pressure was required to open MscL in fatter bilayers (with 20-carbon chains, PC20). This change of MscL gating with the thickness of the membrane formed is called hydrophobic mismatch due to the mismatch of interactions between the membrane and the protein. ${ }^{63,80}$

EPR spectroscopy of the MscL incorporated into thinner PC bilayers with carbon chains of 10 to 16 carbons (PC10-PC16) seemed to indicate a slightly altered structure from the closed state. This altered structure, however, still appeared to have a closed pore. This was proposed to be an intermediate state in the transition from closed to open MscL. Based on this data, the transition from the closed to the intermediate state was proposed to represent a significant energy barrier; this is due to the fact that the proposed intermediate state of MscL found in the thin bilayers is able to be opened at significantly lower pressures than the closed state. This is consistent with the rate limiting step proposed by Sukharev et al. ${ }^{61,80}$ To form the fully open state of MscL hydrophobic mismatch alone was not sufficient, so a different approach involving distortion of the bilayer was attempted. When a lysophosphatidylcholine (LPC) was added to the PC liposome membrane, it disrupted the normal membrane resting state thus driving MscL to the open state. The LPC has been described as a 'cone-shaped' lipid due to its tendency to form micelles, whereas the phospholipids that form bilayers are described as rods (these shapes are approximations based on behaviour rather than actual descriptions of shape). The LPC disrupts the membrane by its ability to distort the lipid bilayer shape causing asymmetric lateral pressure between the two leaflets in the bilayer (Figure 2.3), thus triggering MscL to open. ${ }^{79,80,82}$

Since these developments, much work has been done to improve our knowledge of the mechanism of MscL, as it is still not well understood. ${ }^{83-89}$ Mechanisms have been proposed and adapted as new information has come to light, but our current understanding of MscL is insufficient to fully describe its gating mechanism. This further highlights the need to find a compound that is capable of opening MscL by a direct interaction with the protein in order to better understand its gating mechanism. For recent reviews on 


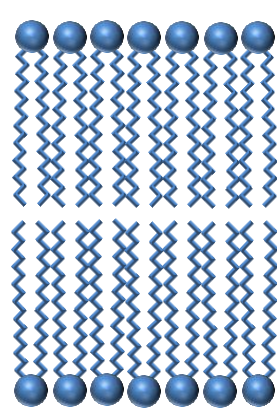

A

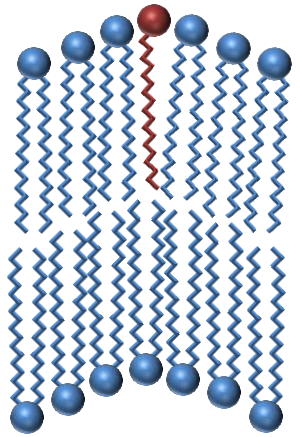

B

Figure 2.3. When an LPC molecule (red) is added to a lipid bilayer (A) it causes it to distort (B) resulting in asymmetric lateral pressure between the two layers, this triggers the opening of MscL.

the research into MscL gating and the conclusions that have been drawn see Booth and Blount (2012) $)^{90}$, Haswell et al. (2011) ${ }^{91}$ and Iscla and Blount (2012). ${ }^{92}$ For a review on the research and proposed mechanisms prior to the revision of the Tb-MscL crystal structure in 2007 see Perozo (2006). ${ }^{63}$

\subsection{MscL Bioassay}

A new bioassay has been developed by Professor Boris Martinac and colleagues at the Victor Chang Cardiac Research Institute. This assay is used to test the activity of compounds on the gating of Ec-MscL that has been reconstituted into azolectin liposomes containing carboxyfluorescein (a self-quenching, fluorescent material). The aim of this project was to screen our library of marine invertebrate extracts against these MscL-containing liposomes to find compounds capable of locking the channel in the open conformation. If the channel were able to be locked in the open conformation in pathogenic bacterial cells, then these cells would not be able to maintain their cellular homeostasis resulting in the slowing down of cell processes and eventually death.

This bioassay was still in the developmental stages and the protocol needed to be adapted to allow the assay to be performed using the equipment available at Victoria University of Wellington (VUW) and Environmental Science and Research (ESR), Kenepuru. The assay was conducted under the supervision of Dr. Penny Truman (ESR). The fragility of the azolectin liposomes meant that they needed to be prepared the same day that the assay was performed. They were also very sensitive to the conditions used to make them, so their preparation needed to be optimised before the assay could be run. Consistent and reproducible results were required from this assay before it could be tested against the library of extracts. 
In this assay, the liposomes were made with carboxyfluorescein incorporated into them. They were then passed through a lipid extruder to allow the collection of liposomes of the desired size (in this case $400 \mathrm{~nm}$ liposomes were used). MscL protein suspended in detergent was then added to these liposomes and allowed to incorporate into the membranes. MscL had been isolated from E. coli and suspended in detergent by Prof. Martinac's group who kindly supplied this for our use. Once the liposomes containing reconstituted MscL were made, they could be tested. As a positive control 1-oleoyl-2-hydroxy-sn-glycero-3-phosphocholine (12), an LPC was used to open the MscL channel (the mechanism of this was discussed in Section 2.4.3). As a measure for the total releasable carboxyfluorescein possible from the liposomes, a 10\% Triton $^{\mathrm{TM}}$ $\mathrm{X}-100$ solution was used as this causes complete lysis of the liposomes.

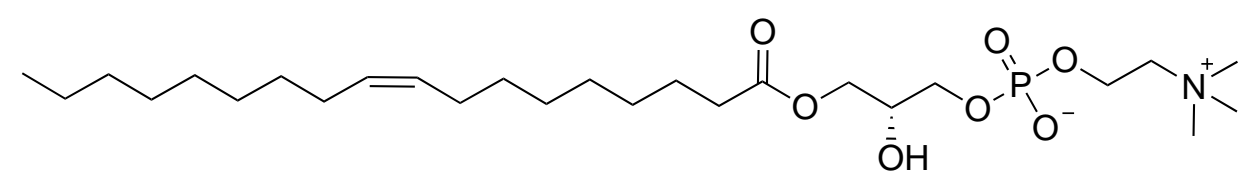

12

The assay worked by measuring the fluorescence of the liposomes in the presence of different compounds. CF being a self-quenching fluorescing agent meant that when the $\mathrm{CF}$ molecules were in the liposomes the molecules would be in a concentrated environment leading to lower fluorescence being observed. When the CF molecules were released from the liposomes they would be in a more dilute environment and so more fluorescence would be observed. The assay was performed in a 96-well black plate and the fluorescence of the wells was read using a fluorescence plate reader (fluorometer). What was observed in the assay was that a solvent control showed the least fluorescence, the liposomes with the LPC added showed an intermediate amount of fluorescence (due to some carboxyfluorescein molecules being able to escape from the liposome) and the liposomes exposed to the $10 \%$ Triton $^{\mathrm{TM}}$ solution showed the highest fluorescence (due to the all the carboxyfluorescein in the liposomes being released into the well).

LPC is able to be used as a positive control due to its ability to distort the membrane. It works by altering the transbilayer pressure profile in a cell or liposome membrane triggering MscL to open. ${ }^{93}$ However, because it works by incorporating into the membrane and distorting it (resulting in the opening of MscL), the LPC molecules will eventually equilibrate by inserting themselves in both layers of the membrane, thus cancelling out the effect of distortion on the membrane and allowing the MscL to close again. ${ }^{93,94}$ For this reason, the current research aims were to find a compound that interacts directly with the MscL protein, locking it in an open state so that it is irreversibly opened. Such a compound would be of use as a more reliable positive control for future assays. It would also act as a probe to allow further studies to be performed on the structure and mechanism of MscL, as well as being a potential antibacterial drug lead. 


\section{Chapter 3}

\section{Screening}

\subsection{Antifungal Assay}

Antifungal assays were chosen because of the readily available resources and facilities for performing this assay with the Chemical Genetics group (in the School of Biological Sciences at VUW) who were able to provide assistance with screening and dose-response assays. The resources were also available to do testing on yeast gene deletion mutant libraries to determine the mode of action of antifungal compounds isolated, however these assays could only be performed if large masses (e.g. $>20 \mathrm{mg}$ ) of these compounds had been isolated. The screening assays were performed in liquid culture on 96-well plates with each extract dissolved in 50\% DMSO in $\mathrm{H}_{2} \mathrm{O}$ and added to the culture. Only one well was used to test each extract in the assay. Positive controls, growth controls, solvent controls and media controls were also set out on each assay plate for accurate comparisons between plates and across assays. The screening assay was performed twice so that each extract was initially tested for activity in duplicate. The dose-response assay used to validate the 'hits' from the screening assays were also performed in liquid culture using 96-well plates and the same controls were applied. The extracts were tested in triplicate with the highest concentration of wells being $100 \mu \mathrm{g} / \mathrm{mL}$ and all subsequent wells being diluted by $50 \%$ (e.g. concentrations across the plate were: $100 \mu \mathrm{g} / \mathrm{mL}$, $50 \mu \mathrm{g} / \mathrm{mL}, 25 \mu \mathrm{g} / \mathrm{mL}$ etc.). The plates for both assays were incubated for 18 hours and then yeast growth was determined using optical density (OD) measurements.

The extract library of New Zealand and Tongan marine invertebrates was tested for activity that inhibits the growth of the yeast Saccharomyces cerevisiae at a concentration of $100 \mu \mathrm{g} / \mathrm{mL}$. If an extract inhibited yeast growth by at least $35 \%$ it was considered to be a 'hit'. Extract 'hits' were then validated in a dose-response assay against $S$. cerevisiae. From this work two sponges of interest were initially identified and bulk extractions done. The sponge MNP_0999 showed very good inhibitory activity with more than $90 \%$ 
inhibition of yeast growth at a concentration of $12.5 \mu \mathrm{g} / \mathrm{mL}$. The sponge MNP_1001 showed moderate inhibitory activity with more than $90 \%$ inhibition at a concentration of $50 \mu \mathrm{g} / \mathrm{mL}$. These sponge extracts were then purified using chromatography. Later in the project an ascidian and sponge that were hits in the initial screens were also extracted and purified. The ascidian PTN3_40G showed good inhibitory activity in the initial screens (with an average inhibition of $88 \%$ at a concentration of $100 \mu \mathrm{g} / \mathrm{mL}$ ) but no activity in the dose-response validation. The sponge PTN2_67C showed weaker inhibitory activity in both the initial screening (with an average inhibition of $50 \%$ at a concentration of $100 \mu \mathrm{g} / \mathrm{mL}$ ) and validation assays. It was hoped that weak activity that had been observed would become stronger upon purification of these extracts.

In the current work several different types of chromatographic media were used for purification. Reversed-phase HP20, poly(styrene-divinylbenzene) stationary support, was used in the first step of purification of the crude extract. It was used by cyclic loading the crude extract on to the HP20 column and eluting the column with the relevant fractions of acetone in water to gain the desired separation. This was followed by the use of HP20ss, which was eluted using fractions of methanol in water and followed by an acetone strip to reduce the risk of sample and activity loss. However, it should be noted that even using 100\% acetone to strip the HP20 and HP20ss columns will not eliminate the risk of sample and activity loss completely. ${ }^{95}$ It has been shown that separation using silica gel can lead to a significant loss of mass and bioactivity in certain natural product extracts, for this reason a Diol bonded phase was used instead for normal-phase separation. ${ }^{95}$ When further purification was required after HP20ss, Diol was often used. Diol columns were eluted with stepped gradients, starting with ethyl acetate in hexanes through ethyl acetate to a gradient of methanol in ethyl acetate until $100 \%$ methanol was reached. The column was finally stripped with $25 \%$ and $50 \%$ water in methanol. For further purification after the use of Diol (or after HP20ss if Diol was not used), HPLC was used in normal (Diol) or reversed (C18) phase modes. The conditions for these HPLC separations were optimised for each compound or group of compounds.

\subsubsection{MNP_0999}

MNP_0999 (Figure 3.1) was a dark-brown, brittle sponge identified as Haliclona sp. that was collected off Port Hardy, d'Urville Island, New Zealand in April 2000 and stored frozen. In validation assays against S. cerevisiae MNP_0999 had a 94\% inhibition at $12.5 \mu \mathrm{g} / \mathrm{mL}$ but showed no inhibition at $6.25 \mu \mathrm{g} / \mathrm{mL}$. This gave an approximate $\mathrm{IC}_{50}$ of $11.0 \mu \mathrm{g} / \mathrm{mL}$ with a large margin of error. A mass of $189 \mathrm{~g}$ of this sponge was extracted with methanol twice and cyclic loaded onto HP20. The MNP_0999 extract was then eluted from the HP20 using 20\%, 40\%, 60\%, $80 \%$ and $100 \%$ acetone in water, these fractions were then backloaded and eluted with acetone to remove water from the fractions. The 


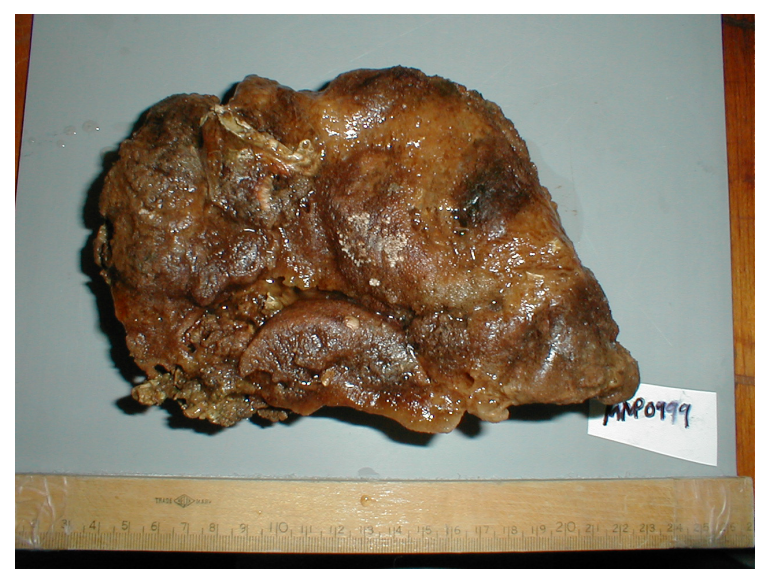

Figure 3.1. Surface photograph of MNP_0999 collected off Port Hardy, d'Urville Island, New Zealand. Photograph courtesy of Associate Professor Peter Northcote.

20\% (VD1_96A) and 40\% (VD1_96B) acetone in water fractions had masses of $128 \mathrm{mg}$ and $388 \mathrm{mg}$, respectively. These samples both had $\mathrm{IC}_{50}$ values of $1.5 \pm 1 \mu \mathrm{g} / \mathrm{mL}$ when tested against $S$. cerevisiae and showed similar ${ }^{1} \mathrm{H}$ NMR spectra (Figure 3.2). Further purification of VD1_96A was done using HP20ss followed by Diol and finally HPLC was used several times to isolate three 3-alkyl pyridinium alkaloid (3-APA) monomers. Two of the 3-APAs isolated have not been previously reported in the literature and one is a known compound. Further purification of VD1_96B was done using HP20ss followed by reversed-phase (C18) HPLC. This produced a 3-APA that was not able to be fully characterised. The isolation and structural elucidation of the 3-APAs will be discussed in Chapter 4.

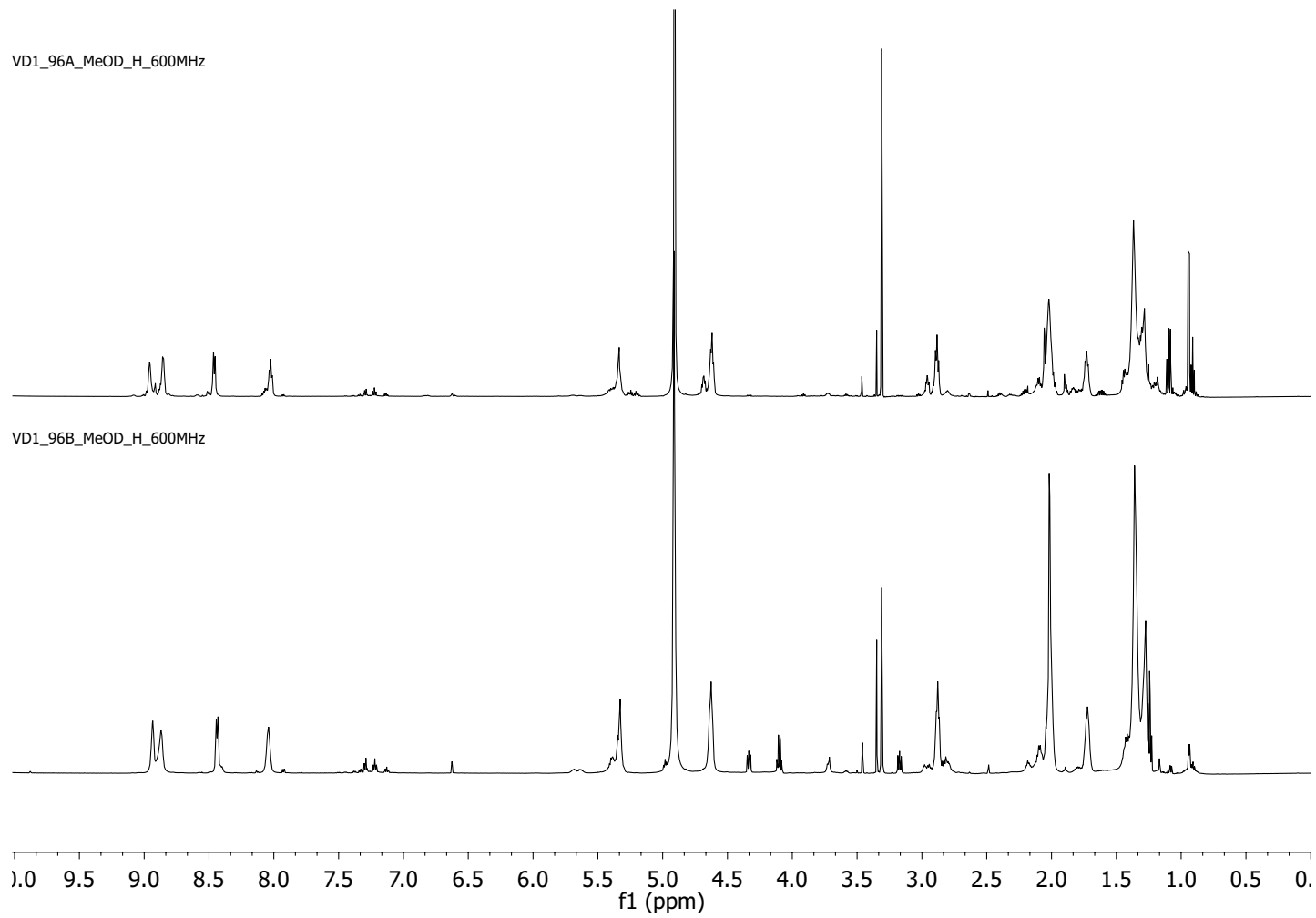

Figure 3.2. ${ }^{1} \mathrm{H}$ NMR spectra of VD1_96A and VD1_96B $\left(600 \mathrm{MHz}, \mathrm{CD}_{3} \mathrm{OD}\right)$. 


\subsubsection{MNP_1001}

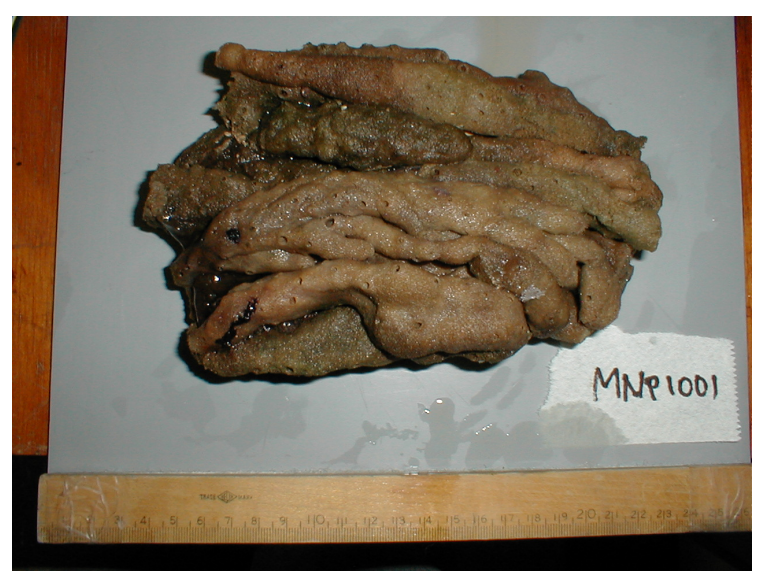

Figure 3.3. Surface photograph of MNP_1001 collected off Port Hardy, d'Urville Island, New Zealand. Photograph courtesy of Associate Professor Peter Northcote.

MNP_1001 (Figure 3.3) was a soft, light brown-green sponge, finger-like in shape. It has been identified as Callyspongia sp. and was collected off Port Hardy, d'Urville Island, New Zealand in April 2000 and stored frozen. In initial validation assays against S. cerevisiae, MNP_1001 inhibited $99 \%$ of yeast growth at a concentration of $50 \mu \mathrm{g} / \mathrm{mL}$ but only $24 \%$ inhibition was observed at a concentration of $25 \mu \mathrm{g} / \mathrm{mL}$. This gave an approximate $\mathrm{IC}_{50}$ of $26.6 \mu \mathrm{g} / \mathrm{mL}$ with a large margin of error. A mass of $112 \mathrm{~g}$ of this sponge was extracted twice with methanol and purified on HP20 using the same procedure used for MNP_0999, very low masses were recovered from this fractionation in the range of 4.9 to $72.3 \mathrm{mg}$. These fractions were tested against $S$. cerevisiae where they showed no significant inhibition. Less than $10 \%$ inhibition was observed in all fractions at concentrations of $200 \mu \mathrm{g} / \mathrm{mL}$ (the highest concentration tested). This assay was repeated to ensure that the compounds were not active before work on this sponge was ceased in favour of other, more promising, leads. The repeat assay included testing the activity of the recombined fractions in case the mechanism of inhibition was synergistic. This recombined fraction, however, showed no activity either and so this isolation is likely to be an example of the active component of an extract irreversibly binding to HP20. ${ }^{95}$ The lack of activity of this sponge in combination with the low masses recovered meant that work on this sponge was not pursued further.

\subsubsection{PTN3_40G}

PTN3_40G (Figure 3.4) was an unidentified ascidian that had been collected at Swallows Cave in Vava'u, Tonga in November 2009. Only $17.92 \mathrm{~g}$ of this ascidian had been collected and so the entire mass was extracted in methanol. The solution was a dark orange-red colour. In initial validation assays against $S$. cerevisiae, PTN3_40G showed weak inhibition with $65 \%$ inhibition of yeast growth at a concentration of $100 \mu \mathrm{g} / \mathrm{mL}$ (the 


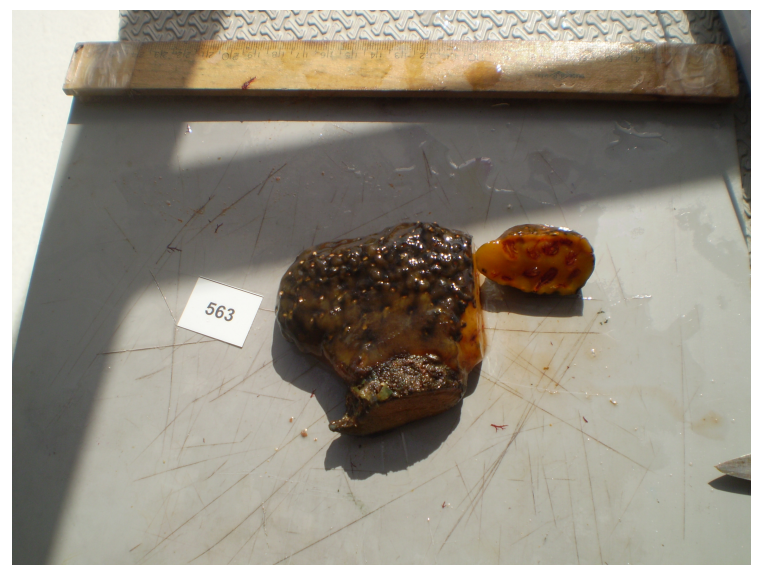

Figure 3.4. Surface photograph of PTN3_40G collected at Swallows Cave in Vava'u, Tonga. Photograph courtesy of Associate Professor Peter Northcote.

highest concentration tested). PTN3_40G was cyclic loaded on to HP20 and eluted using $30 \%, 75 \%$ and $100 \%$ acetone in water. The fractions containing water were backloaded on to HP20 and eluted to remove the water. The 75\% acetone in water fraction (VD3_64B) had a mass of $51.1 \mathrm{mg}$ and was shown to inhibit $27 \%$ of yeast growth at a concentration of $100 \mu \mathrm{g} / \mathrm{mL}$ when tested against $S$. cerevisiae. This fraction was further purified on HP20ss and tested for activity against $S$. cerevisiae again. Two fractions collected from this HP20ss column from 80\% methanol in water (VD3_80G and VD3_80H) showed some inhibition in the assay with a $35 \%$ and $76 \%$ inhibition of growth observed at a concentration of $100 \mu \mathrm{g} / \mathrm{mL}$ for VD3_80G and VD3_80H, respectively. However, no inhibition was observed at a concentration of $50 \mu \mathrm{g} / \mathrm{mL}$. These fractions were less than $4 \mathrm{mg}$ in mass each and so were not practical to continue purification from. Instead, the acetone strip of the column (VD3_80C), which showed no activity against $S$. cerevisiae, was further purified on Diol followed by Diol HPLC because of its mass of $14.1 \mathrm{mg}$ and the ${ }^{1} \mathrm{H}$ NMR spectrum showing several signals of interest. From the first Diol column, VD3_98G was isolated from 50\% ethyl acetate in hexanes. A full set of NMR experiments $\left({ }^{1} \mathrm{H},{ }^{13} \mathrm{C}, \mathrm{COSY}, \mathrm{HSQC}\right.$ and $\left.\mathrm{HMBC}\right)$ were run on this compound and these were used to propose an unsaturated fatty acid structure. VD3_98E, which was also isolated from the $50 \%$ ethyl acetate in hexanes fraction of the Diol column, was further purified using Diol HPLC. A full set of NMR experiments were run on this purified fraction (VD4_17B) and these were used to propose a phthalate ester structure. The isolation of these compounds from PTN3_40G is shown in Scheme 3.1.

\section{VD3988G}

The ${ }^{1} \mathrm{H}$ NMR spectrum of VD3_98G (Figure 3.5) comprised of an alkene triplet at $5.35 \mathrm{ppm}$, a methylene triplet at $2.22 \mathrm{ppm}$, a methylene doublet at $2.01 \mathrm{ppm}$, a methylene quintet at $1.63 \mathrm{ppm}$, a methylene envelope from 1.24 to $1.34 \mathrm{ppm}$ and a methyl triplet at $0.88 \mathrm{ppm}$. The ${ }^{13} \mathrm{C}$ NMR spectrum of VD3_98G (Figure 3.6) contained a carbonyl 


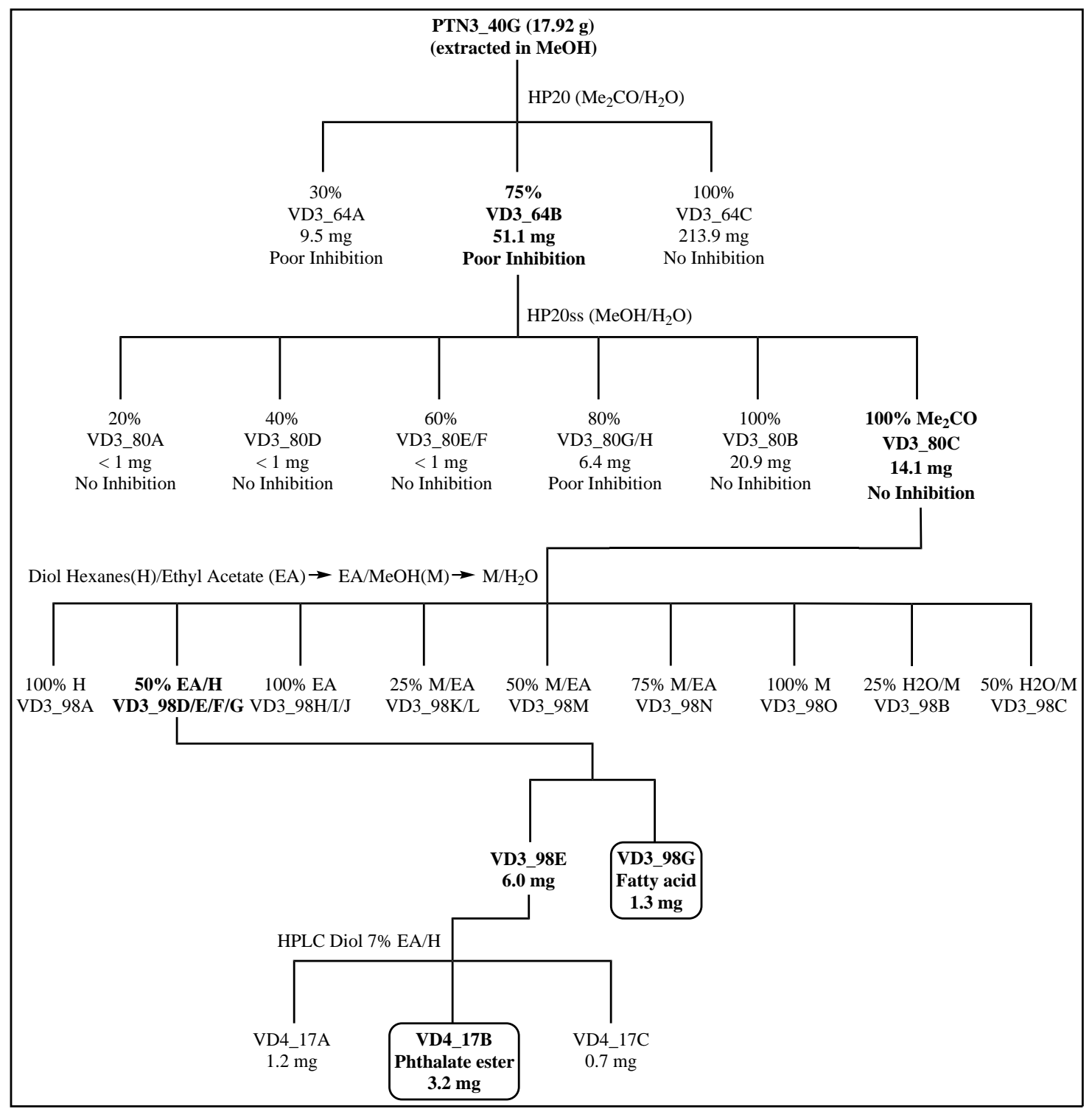

Scheme 3.1. Isolation procedure for unsaturated fatty acid (VD3_98G) and phthalate ester (VD4_17B) from the sponge PTN3_40G.

resonance at $175.6 \mathrm{ppm}$, alkene resonances at 130.1 and $130.0 \mathrm{ppm}$, several methylene resonances between 36.1 and $22.9 \mathrm{ppm}$ and a methyl resonance at $14.3 \mathrm{ppm}$. These assignments were consistent with HSQC data and suggested a fatty acid structure. This was further supported by the COSY spectrum that showed the methyl proton resonance coupling to the methylene envelope, the alkene protons coupling to the resonance at $2.01 \mathrm{ppm}$ that further coupled to the methylene envelope, and the methylene triplet at $2.22 \mathrm{ppm}$ coupling to the quintet at $1.63 \mathrm{ppm}$ that further coupled to the methylene envelope. The HMBC showed correlations from the protons at 2.22 and $1.63 \mathrm{ppm}$ to the carbonyl carbon and correlations from the protons at $2.01 \mathrm{ppm}$ and the methylene envelope to the alkene carbons, which further supported this proposal. The MS data, however, was not able to give a clear molecular ion for this compound consistent with any of the fatty acid structural possibilities proposed based on the NMR information, this prevented the conclusive determination of this structure. $\mathrm{A}_{18}$ alkyl chain length of the fatty acid was proposed based on the number of methylene groups that could be 
distinguished in the ${ }^{13} \mathrm{C}$ NMR spectrum. Comparison of the ${ }^{1} \mathrm{H}$ and ${ }^{13} \mathrm{C}$ NMR data of this fatty acid with some well known $\mathrm{C}_{18}$ fatty acids allowed this compound to be tentatively assigned as cis-vaccenic acid (13).
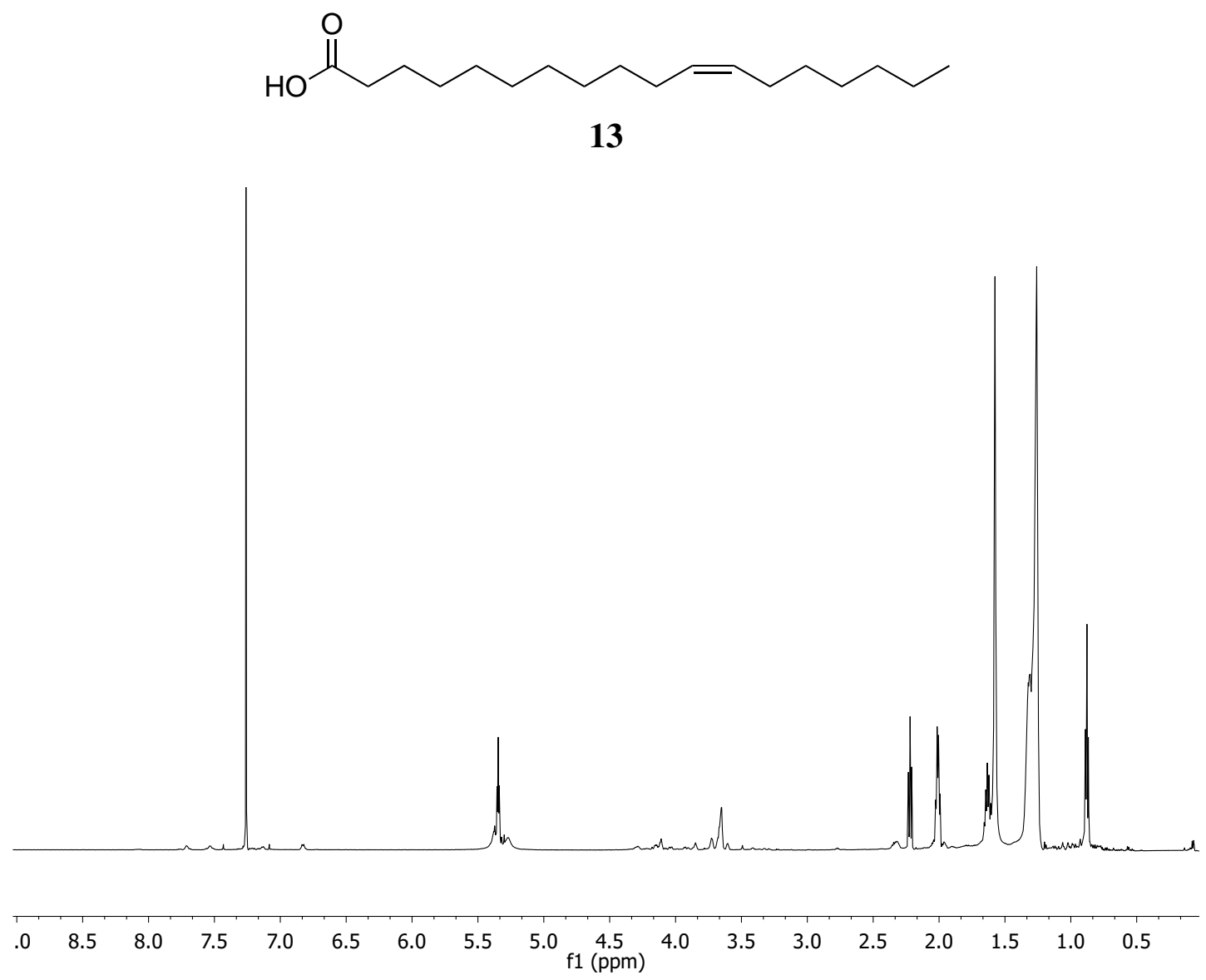

Figure 3.5. ${ }^{1} \mathrm{H}$ NMR spectrum of VD3_98G $\left(600 \mathrm{MHz}, \mathrm{CDCl}_{3}\right)$.

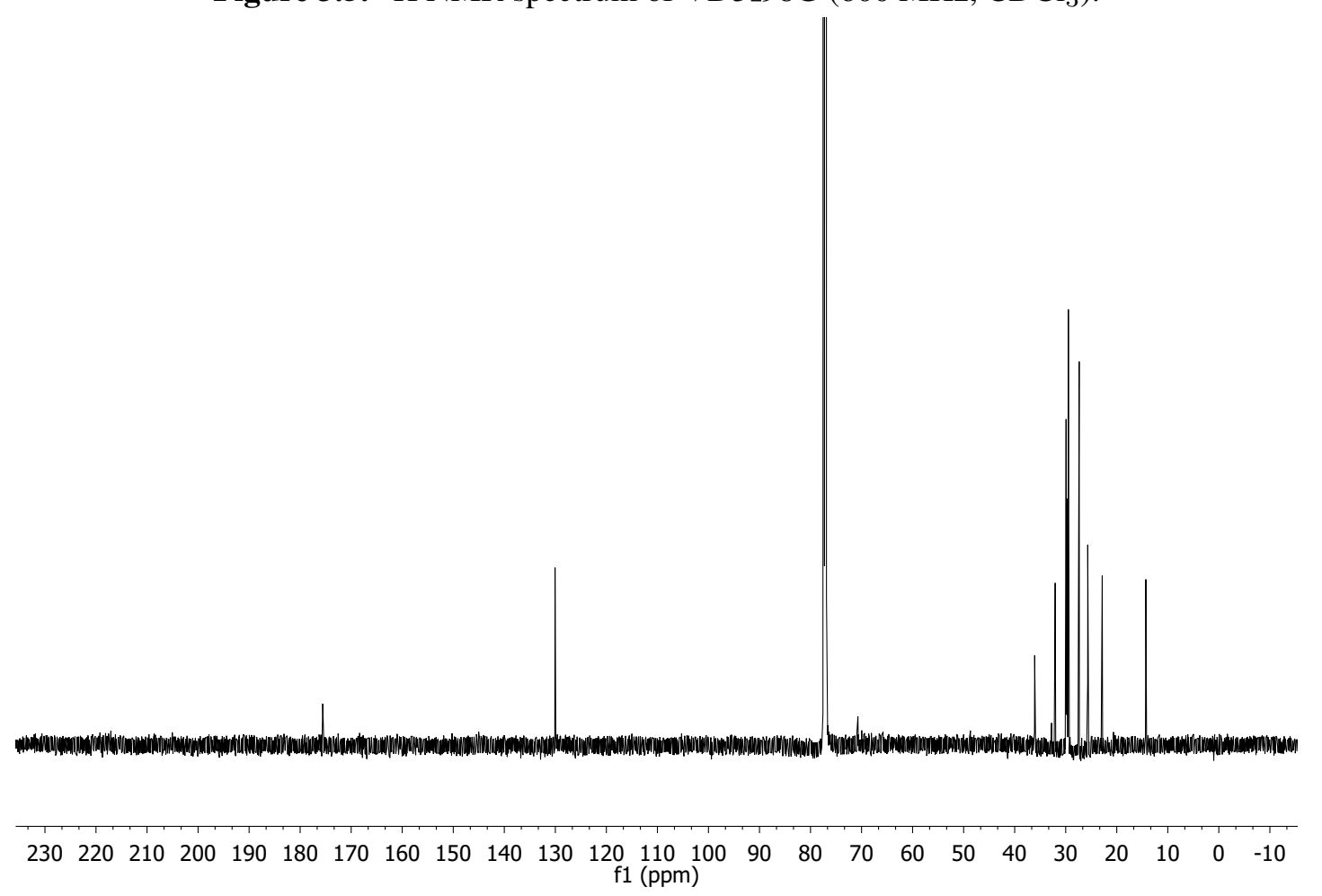

Figure 3.6. ${ }^{13} \mathrm{C}$ NMR spectrum of VD3_98G $\left(150 \mathrm{MHz}, \mathrm{CDCl}_{3}\right)$. 
VD4_17B was isolated as a mix of very similar compounds that eluted at the same time; this was indicated by a single peak in the UV/Vis trace on the HPLC and the many overlapping resonances that occurred in the NMR spectra. The ${ }^{1} \mathrm{H}$ NMR spectrum of VD4_17B (Figure 3.8) contained two highly second order multiplets that appeared to be overlapping doublets or doublets of doublets at 7.73-7.71 ppm and 7.63-7.61 ppm that were characteristic of a 1,2-disubstituted benzene ring. There was also a multiplet of overlapping resonances around 4.35-4.26 ppm as well as many overlapping resonances in the methylene and methyl regions of the ${ }^{1} \mathrm{H}$ NMR spectrum. The ${ }^{13} \mathrm{C}$ NMR spectrum (Figure 3.9) contained a broad carbonyl resonance at $167.9 \mathrm{ppm}$ that was characteristic of an ester and aromatic resonances at 132.1, 130.9 and a broad resonance at $128.4 \mathrm{ppm}$ that could be attributed to a symmetrical benzene ring. There were several ${ }^{13} \mathrm{C}$ resonances from 65.8 to $63.8 \mathrm{ppm}$, the major resonance being $65.8 \mathrm{ppm}$, which was likely to be due to a carbon attached to oxygen in an ester functionality. The ${ }^{13} \mathrm{C}$ NMR spectrum also contained many resonances between 46.5 and $10.4 \mathrm{ppm}$ that were characteristic of methylene and methyl carbons. The HMBC spectrum of VD4_17B showed the two aromatic proton resonances correlating to the carbonyl carbon and the other aromatic carbons. It also showed the resonances between 4.35 and $4.26 \mathrm{ppm}$ correlating to the carbonyl carbon and the methylene carbon resonances. This information indicated that a mixture of phthalate esters had been isolated. Further purification using HPLC would allow the individual phthalate esters to be separated out and individually identified, however, the lack of activity observed and the well-known nature of these compounds meant that this sample was not pursued further. It should, however, be noted that biologically active, novel phthalate esters have been isolated from marine organisms previously. ${ }^{96,97}$<smiles>[R]COC(=O)c1ccccc1C(=O)OC[R]</smiles>

Figure 3.7. A phthalate ester structure $(\mathrm{R}=$ saturated alkyl chains of unknown length in VD4_17B). 


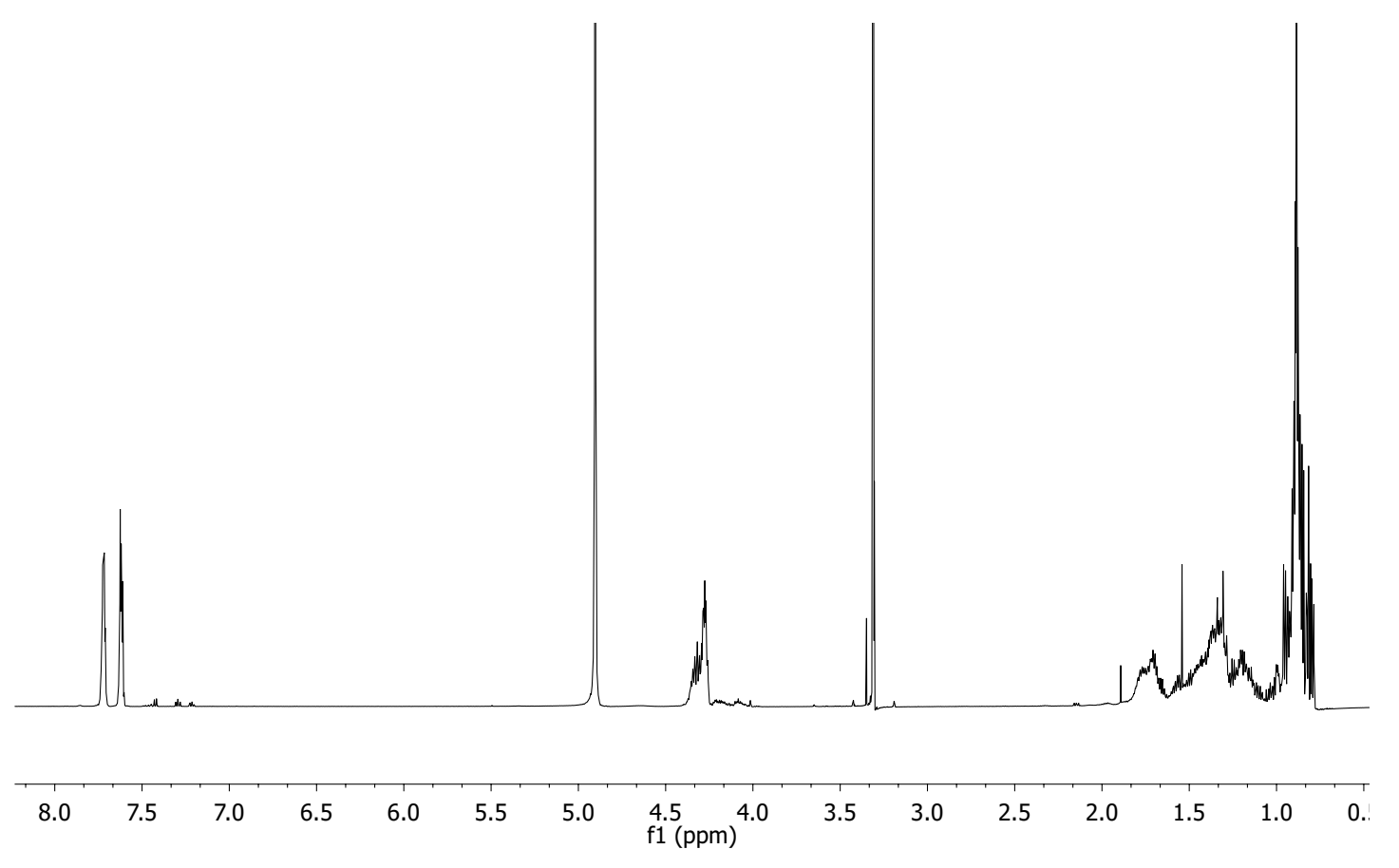

Figure 3.8. ${ }^{1} \mathrm{H}$ NMR spectrum of VD4_17B $\left(600 \mathrm{MHz}, \mathrm{CD}_{3} \mathrm{OD}\right)$.

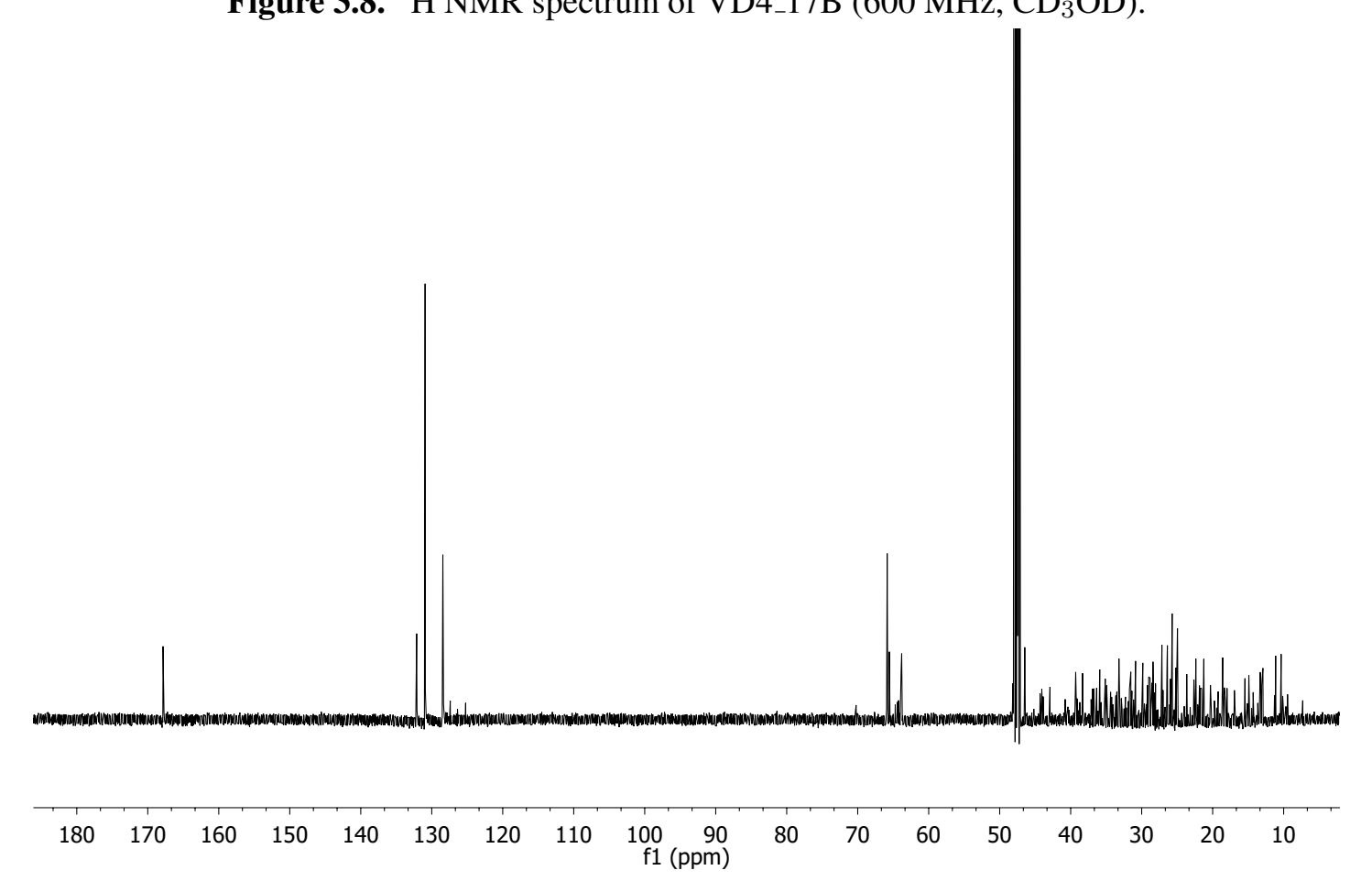

Figure 3.9. ${ }^{13} \mathrm{C}$ NMR spectrum of VD4_17B $\left(150 \mathrm{MHz}, \mathrm{CD}_{3} \mathrm{OD}\right)$.

\subsubsection{PTN2_67C}

PTN2_67C (Figure 3.10) was a soft, cream-coloured sponge that consisted of many small, tightly packed tube-like structures. It was a calcareous sponge collected at Cape Karikari in New Zealand in December 2003 and stored frozen. This sponge has been tentatively identified as Leucosolenia sp. In validation assays against S. cerevisiae PTN2_67C showed weak activity with approximately $30 \%$ inhibition of the growth of yeast at a concentration of $100 \mu \mathrm{g} / \mathrm{mL}$ (the highest concentration tested). A mass of $93 \mathrm{~g}$ of this 


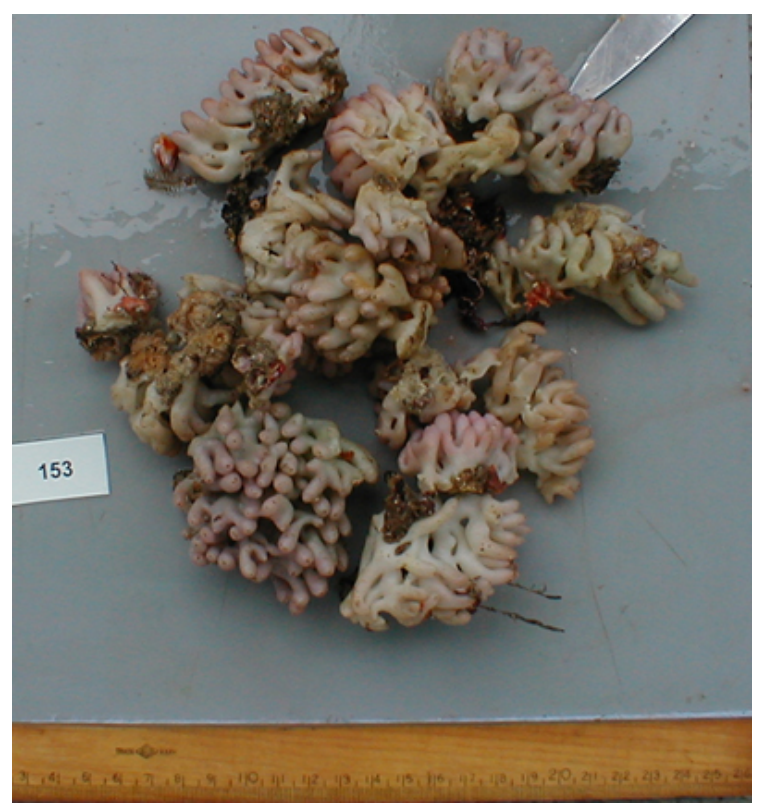

Figure 3.10. Surface photograph of PTN2_67C collected at Cape Karikari, New Zealand. Photograph courtesy of Associate Professor Peter Northcote.

sponge was extracted twice with methanol and this extract was purified on HP20 following the same procedure used for PTN3_40G. The 30\% acetone in water fraction (VD3_68A) had a mass of $30.5 \mathrm{mg}$ and the $75 \%$ fraction (VD3_68B) had a mass of $185.5 \mathrm{mg}$. When tested against $S$. cerevisiae, VD3_68A inhibited the growth of yeast by $86 \%$ at a concentration of $25 \mu \mathrm{g} / \mathrm{mL}$ and by $13 \%$ at $12.5 \mu \mathrm{g} / \mathrm{mL}$, this gave an $\mathrm{IC}_{50}$ of $16 \pm 1 \mu \mathrm{g} / \mathrm{mL}$. VD3_68B inhibited the growth of yeast by $92 \%$ at a concentration of $50 \mu \mathrm{g} / \mathrm{mL}$ and an average of $47 \%$ at a concentration of $25 \mu \mathrm{g} / \mathrm{mL}$. This resulted in an $\mathrm{IC}_{50}$ of $25 \pm 1 \mu \mathrm{g} / \mathrm{mL}$. Purification of VD3_68B was continued while purification of VD3_68A was not because of the much larger mass of VD3_68B. HP20ss was used to further fractionate VD3_68B and these fractions were tested against $S$. cerevisiae again.

The fraction with the highest mass recovered $(30.1 \mathrm{mg})$ and the most activity in the assay was VD3_79B, this was collected in bulk as the $100 \%$ methanol fraction from the HP20 column. VD3_79B inhibited the growth of yeast by $74 \%$ at a concentration of $100 \mu \mathrm{g} / \mathrm{mL}$ and by $25 \%$ at a concentration of $50 \mu \mathrm{g} / \mathrm{mL}\left(\mathrm{IC}_{50} 94 \pm 2 \mu \mathrm{g} / \mathrm{mL}\right.$ ). The $80 \%$ methanol in water fraction from the HP20ss (VD3_79F) also showed some activity with $51 \%$ growth inhibition at a concentration of $100 \mu \mathrm{g} / \mathrm{mL}$ and the $100 \%$ acetone strip (VD3_79C) also showed some minor activity with $31 \%$ growth inhibition at the same concentration. However, the fractions VD3_79F and C had masses of 3.2 and $7.9 \mathrm{mg}$, respectively, and so were not pursued further. VD3_79B was then further purified on Diol and, from the $75 \%$ ethyl acetate in hexanes fraction, several trihydroxylated steroids were isolated. The steroidal fractions were pooled together (VD4_05KLM) and a full set of NMR experiments were run on this. The steroid fraction was then further purified on reversed-phase (C18) HPLC. The steroids had very similar structures and so were difficult to separate on HPLC. A relatively clean fraction (VD5_71J) was chosen and a full set of NMR experiments were run in order to solve the structure of a trihydroxylated steroid. 
This trihydroxylated steroid was proposed to have the structure 14, this compound was not submitted for biological testing due to the small mass recovered $(0.3 \mathrm{mg})$, the low activity of parent fractions and time constraints. The isolation of the trihydroxylated steroid VD5_71J from the sponge PTN2_67C is shown in Scheme 3.2.

VD5_71J

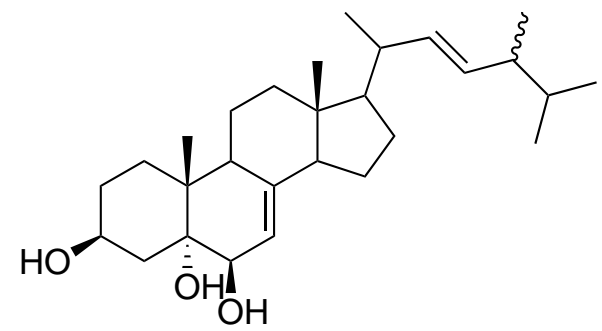

14

The full set of NMR experiments run on the steroids VD4_05KLM showed that the mixture of trihydroxylated steroids isolated only differed in the structure of the side chain. The steroidal resonances of this full set were all the same and the only overlapping resonances of different structures were in the methylene and methyl regions. After HPLC purification the structure of VD5_71J (14), which is a known compound, was solved as a representative structure of the mixture of trihydroxylated steroids that had been isolated. Fraction VD5_71J was chosen because it had the highest mass of the clean fractions and so was the best candidate for getting a full set of NMR data on. The mass of VD5_71J that was isolated after HPLC purification was $0.3 \mathrm{mg}$, this meant that a carbon spectrum was not able to be obtained and all other spectra were relatively weak. Longer NMR experiments were run in order to get as much information as possible for this compound. The ${ }^{1} \mathrm{H}$ NMR of VD5_71J (Figure 3.11) showed a triplet of triplets at $3.97 \mathrm{ppm}(J=4.8,11.4 \mathrm{~Hz})$ that integrated for one proton. This proton was characteristic of a proton at the 3-position of a steroidal structure, with a larger axial-axial coupling of 11.4 Hz to two protons and a smaller axial-equatorial coupling of $4.8 \mathrm{~Hz}$ to two protons. The ${ }^{1} \mathrm{H}$ NMR spectrum also showed the presence of six methyl groups; two methyl singlets at 1.06 and $0.65 \mathrm{ppm}$ and four methyl doublets at 1.04, 0.94, 0.87 and $0.85 \mathrm{ppm}$. The methyl singlets could be attributed to the two angular methyls $\left(\mathrm{CH}_{3}-18\right.$ and $\left.\mathrm{CH}_{3}-19\right)$ of the steroid and the methyl doublets were attributed to the four methyls that appear on the alkyl side chain. There were two alkene multiplet resonances at 5.27 and $5.20 \mathrm{ppm}$ that integrated for one and two protons, respectively. The remaining resonances that could be attributed to the steroid were methylene resonances between 2.12 and $1.29 \mathrm{ppm}$ that were very close together or overlapping. All remaining resonances in the ${ }^{1} \mathrm{H}$ NMR of VD5_71J were due to impurities.

Although the sample VD5_71J was too small to obtain carbon data, the chemical 


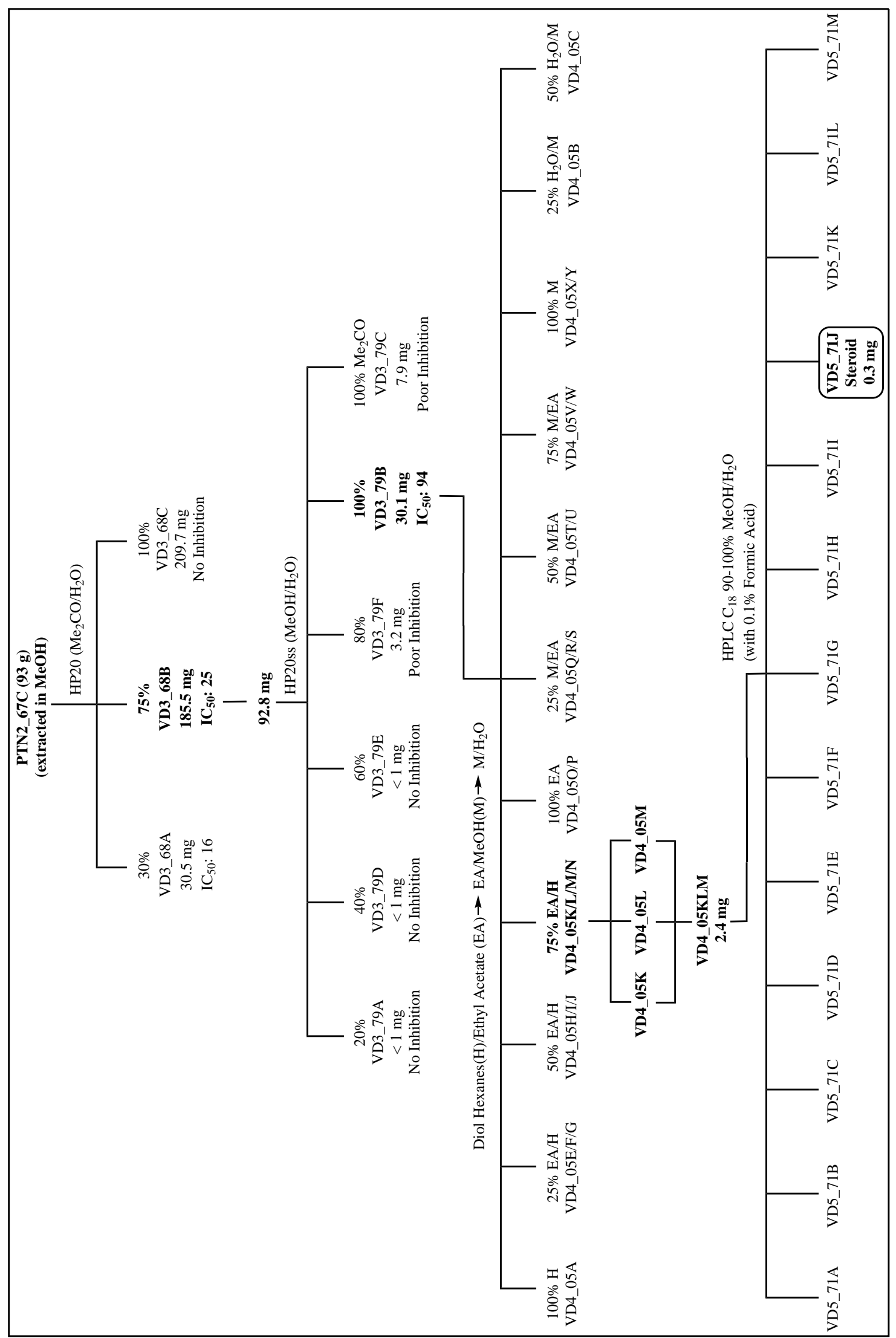

Scheme 3.2. Isolation procedure for the steroid VD5_71J from the sponge PTN2_67C. Note that $\mathrm{IC}_{50}$ values are approximate $(\mu \mathrm{g} / \mathrm{mL})$.

shift information for carbons could be obtained from HSQC and HMBC data. This information was used in combination with the steroid structure assigned from the full set 


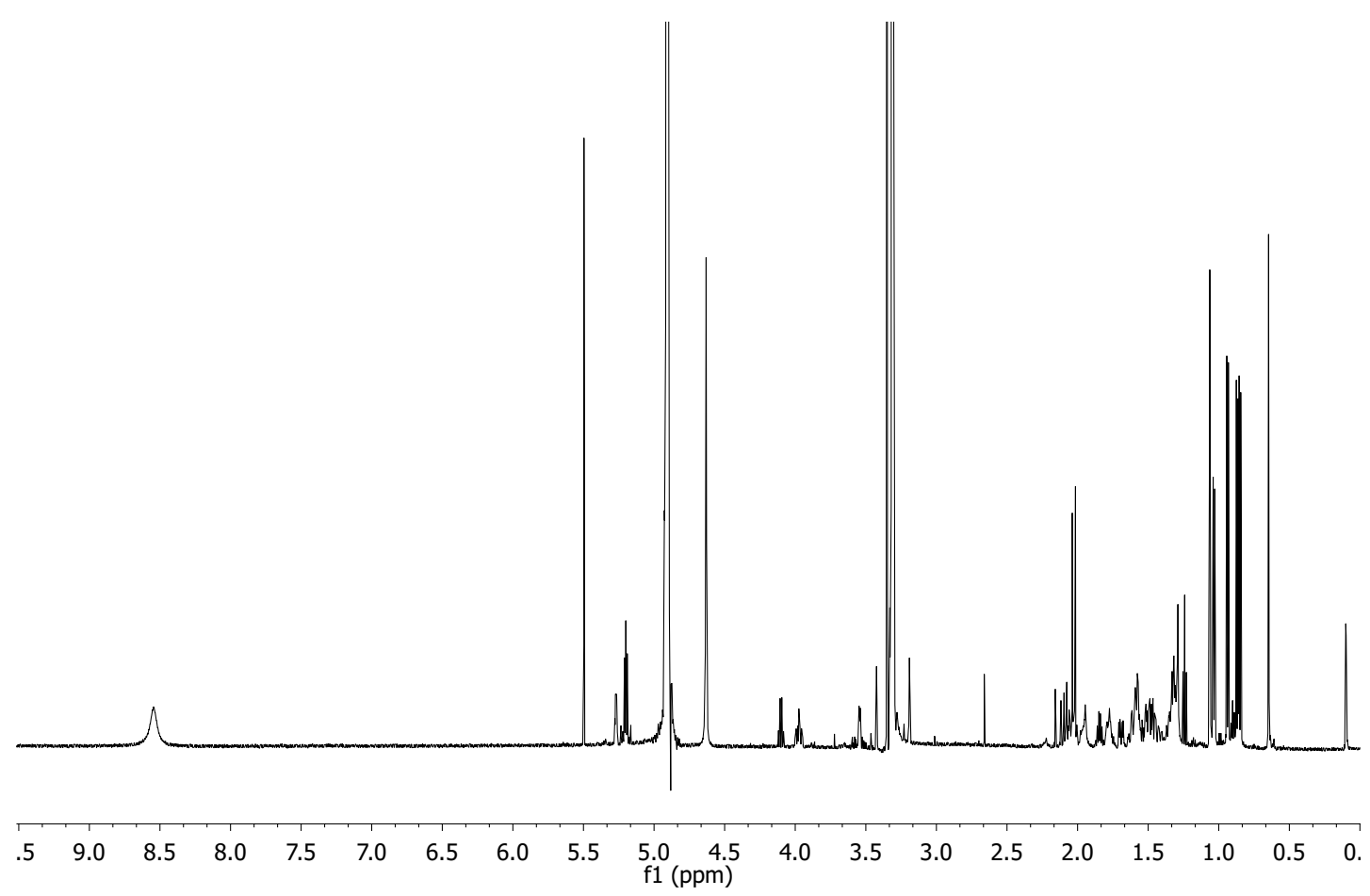

Figure 3.11. ${ }^{1} \mathrm{H}$ NMR spectrum of VD5_71J (600 MHz, $\left.\mathrm{CD}_{3} \mathrm{OD}\right)$.

of VD4_05KLM to propose chemical shifts of the carbons of VD5_71J. It was necessary to assign the chemical shifts from the spectra of VD5_71J instead of from VD4_05KLM as the former NMR experiments were run in $\mathrm{CD}_{3} \mathrm{OD}$ due to being less soluble in $\mathrm{CDCl}_{3}$. The ${ }^{13} \mathrm{C}$ chemical shifts identified from the HSQC and HMBC data of VD5_71J were attributable to three alkene methines $\left(\delta_{\mathrm{C}} 137.0, \mathrm{C}-22 ; 133.0, \mathrm{C}-23 ; 118.8, \mathrm{C}-7\right)$, two oxygenated methines $\left(\delta_{\mathrm{C}} 68.1, \mathrm{C}-3 ; 73.9, \mathrm{C}-6\right)$, six methines $\left(\delta_{\mathrm{C}} 57.0, \mathrm{C}-17 ; 55.6, \mathrm{C}-14\right.$; 44.3, C-24; 44.0, C-9; 41.7, C-20; 34.2, C-25), one oxygenated non-protonated carbon $\left(\delta_{\mathrm{C}} 76.6, \mathrm{C}-5\right)$, two non-protonated carbons $\left(\delta_{\mathrm{C}} 44.4, \mathrm{C}-13 ; 37.8, \mathrm{C}-10\right)$, four methylenes $\left(\delta_{\mathrm{C}} 40.1, \mathrm{C}-4 ; 33.6, \mathrm{C}-1 ; 31.5, \mathrm{C}-2 ; 40.4, \mathrm{C}-12\right)$, and six methyl groups $\left(\delta_{\mathrm{C}} 21.4, \mathrm{C}-21\right.$; 20.4, C-26; 19.8, C-27; 18.6, C-19; 18.4, C-28; 12.4, C-18).

The position of the steroidal alkene and hydroxyl groups were able to be assigned using the impure VD4_05KLM spectra (run in $\mathrm{CDCl}_{3}$ ) in combination with the weaker, pure VD5_71J spectra (run in $\mathrm{CD}_{3} \mathrm{OD}$ ). The A ring structure was as able to be solved using COSY correlations in both sets of spectra as four out of the six ring carbons have hydrogen attached (H-1 to $\mathrm{H}-4)$. Remaining correlations to the non-protonated carbons could be seen in the HMBC correlations of VD4_05KLM, these showed that the C-5 position was oxygenated and the quaternary $\mathrm{C}-10$ position had a methyl attached. The alkene resonance of the cyclic portion could be seen to integrate for only one proton from both sets of spectra (although this was clearer in the ${ }^{1} \mathrm{H}$ NMR spectra of VD5_71J); this indicated that the other end of the alkene was fully substituted. The alkene proton clearly coupled to an oxygenated methine in both COSY spectra (the methine functionality was confirmed by both HSQC spectra). In the impure HMBC spectrum, the oxygenated proton adjacent to the alkene showed correlations to both of the alkene carbons, the C-5 oxygenated 
centre and the quaternary carbon $\mathrm{C}-10$. The alkene proton also correlated to both of the oxygenated centres in this HMBC spectrum. This analysis indicated the A and B ring substructure shown in Figure 3.12, as this is the only possible arrangement of an alkene with one end fully saturated given the structure of the A ring (Figure 3.12).

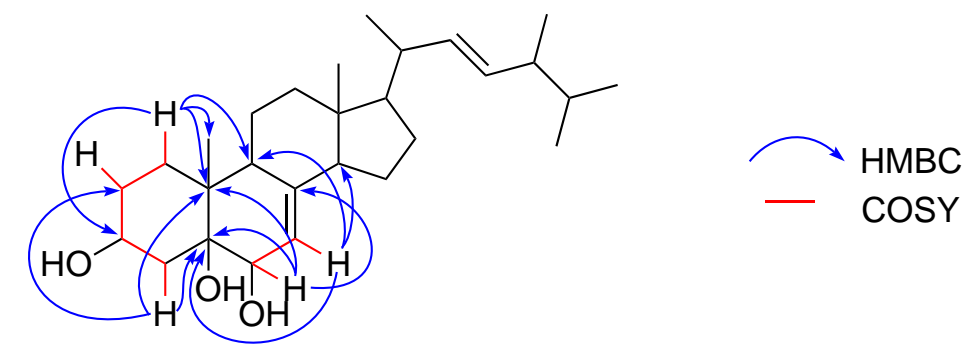

Figure 3.12. Key COSY and HMBC correlations establishing the A and B ring structure of steroid VD5_71J.

The $\mathrm{C}$ and $\mathrm{D}$ rings of the steroid structure were much harder to solve as their correlations were both weak and overlapping. This meant that the D ring structure was not able to be fully assigned from observed correlations. Given the lack of notable functional groups, it was assumed that the $\mathrm{C}$ and $\mathrm{D}$ rings were non-functionalised steroid rings. Some correlations from the $\mathrm{C}$ and $\mathrm{D}$ ring structure could be seen in the impure spectra of VD4_05KLM (Figure 3.13). The methyl groups in both sets of spectra (which show the strongest correlations in the HSQC and HMBC spectra) were able to be used to help assigning the resonances of the $\mathrm{C}$ and $\mathrm{D}$ rings as well as confirming the proposed structure of the A and B ring. Most of the side chain structure was able to be solved using COSY data of VD5_71J and this could then be confirmed by using the HMBC data because of the four methyl groups in the side chain giving characteristic strong correlations. This enabled the full side chain structure proposed from the COSY data to be confirmed (Figure 3.14).

This structure was confirmed using positive ion mode HRESIMS data that showed an $[\mathrm{M}+\mathrm{Na}]^{+}$ion of $\mathrm{m} / \mathrm{z} 453.3305(\mathrm{~m} / \mathrm{z} \Delta=-0.0040)$ that indicated a molecular formula of $\mathrm{C}_{28} \mathrm{H}_{46} \mathrm{O}_{3} \mathrm{Na}$. This steroidal structure has previously been reported from marine sponges and a bryozoan, ${ }^{98-102}$ because of this and limited time, no attempt was made to assign stereochemistry of the C-28 methyl, which shows very similar shifts in both the $R$ and $S$ configurations. ${ }^{98-100,102}$ All other spectroscopic data matched the data in the literature and confirmed this structural assignment. ${ }^{98-102}$
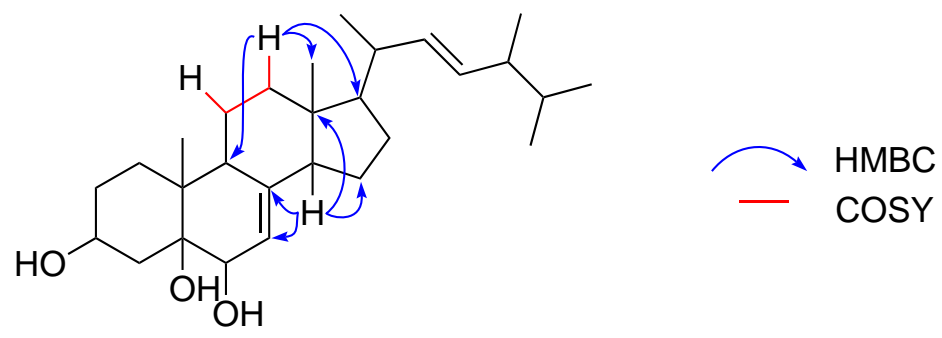

Figure 3.13. Key COSY and HMBC correlations providing evidence for the $\mathrm{C}$ and $\mathrm{D}$ ring structure of steroid VD5_71J. 


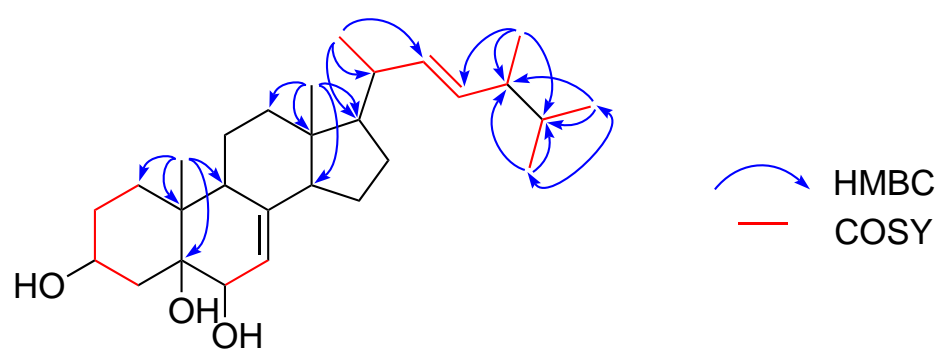

Figure 3.14. Key COSY and HMBC correlations establishing the side chain and structure fragments of steroid VD5_71J. Double headed arrows represent reciprocal HMBC correlations.

\subsection{MscL Assay}

The MscL assay was initially designed as a means of identifying compounds that affect Msc. The assay was used in this study to try and find compounds that can inhibit the growth of bacteria by interacting with the MscL channel. The aim was to lock the MscL channel in the open conformation so that bacteria would be unable to control their homeostasis and this would eventually result in cell death. Alternatively, the MscL being locked in the open conformation would mean that bacteria could not control what enters (as well as leaves) the cell and so a combination approach could be taken where one drug locks the channels open, allowing a second drug to enter the cells and kill the bacteria. In order to find a compound that interacts with MscL, the assay first needed to be optimised for use at VUW and then tested against the extract libraries available, however, the optimisation of the assay proved to be more difficult than expected, resulting in inconsistency across the assays with poor reproducibility.

The carboxyfluorescein-containing liposomes for this assay were made using a thin film of azolectin in carboxyfluorescein (CF) buffer (DR buffer with carboxyfluorescein and $\mathrm{KOH})$. Once the liposomes had been formed using vortexing and sonication, they were forced through a $400 \mathrm{~nm}$ pore membrane using a lipid extruder to give a uniform size. The MscL protein was then incorporated into the liposomes by constant mixing and the liposomes were separated from the unincorporated CF using Sephadex gel filtration. The liposomes collected from the gel filtration were ready to be used in the assay, however, first they needed to be diluted in DR buffer so that a fluorescence difference that is detectable by the fluorometer was achieved. CF is a self-quenching fluorescing agent and so it will fluoresce less at concentrations above a certain threshold compared with lower concentrations, this results in a greater difference in fluorescence observed at concentrations dilute enough to minimise self-quenching behaviours. The fluorescent liposome solutions were transferred to black 96-well plates for the assay so that when the fluorescence was read there would be no transfer of fluorescence detected across the wells. The liposomes made in this work were very fragile and so were only able to be used the same day; most of the liposomes in a solution would have burst by the following day. 


\subsubsection{Optimising Parameters}

A dilution curve of the CF buffer was created (Figure 3.15) in order to determine the optimal fluorescence to be used for the fluorometer and identify the corresponding concentration of CF buffer. This showed that the peak fluorescence was achieved at a concentration of $0.4-0.5 \mathrm{mM}$ and above this concentration self-quenching occurred. This exercise also showed that the level of fluorescence observed could be altered by changing the number of flashes the fluorometer used, however, at higher flash levels (e.g. 100) the shape of the dilution curve was altered, possibly due to saturation of the detector (Figure 3.16). Blank plates were read on the fluorometer in order to determine whether cleaning methods used were sufficient to remove residual CF from the plates. These plates were filled with DR buffer and the fluorescence was read at 10 and 20 flashes. Reading the plates at 10 flashes was not suitable for this assay as fluorescences above zero were found for almost all of the wells, whereas, when using 20 flashes most of the wells had a fluorescence of zero. These inconsistencies in fluorescence in blank plates is undesirable because small fluctuations in the fluorescence reading in an assay could create false positives or negatives, it is for this reason that 20 flashes were used in all subsequent plate readings.

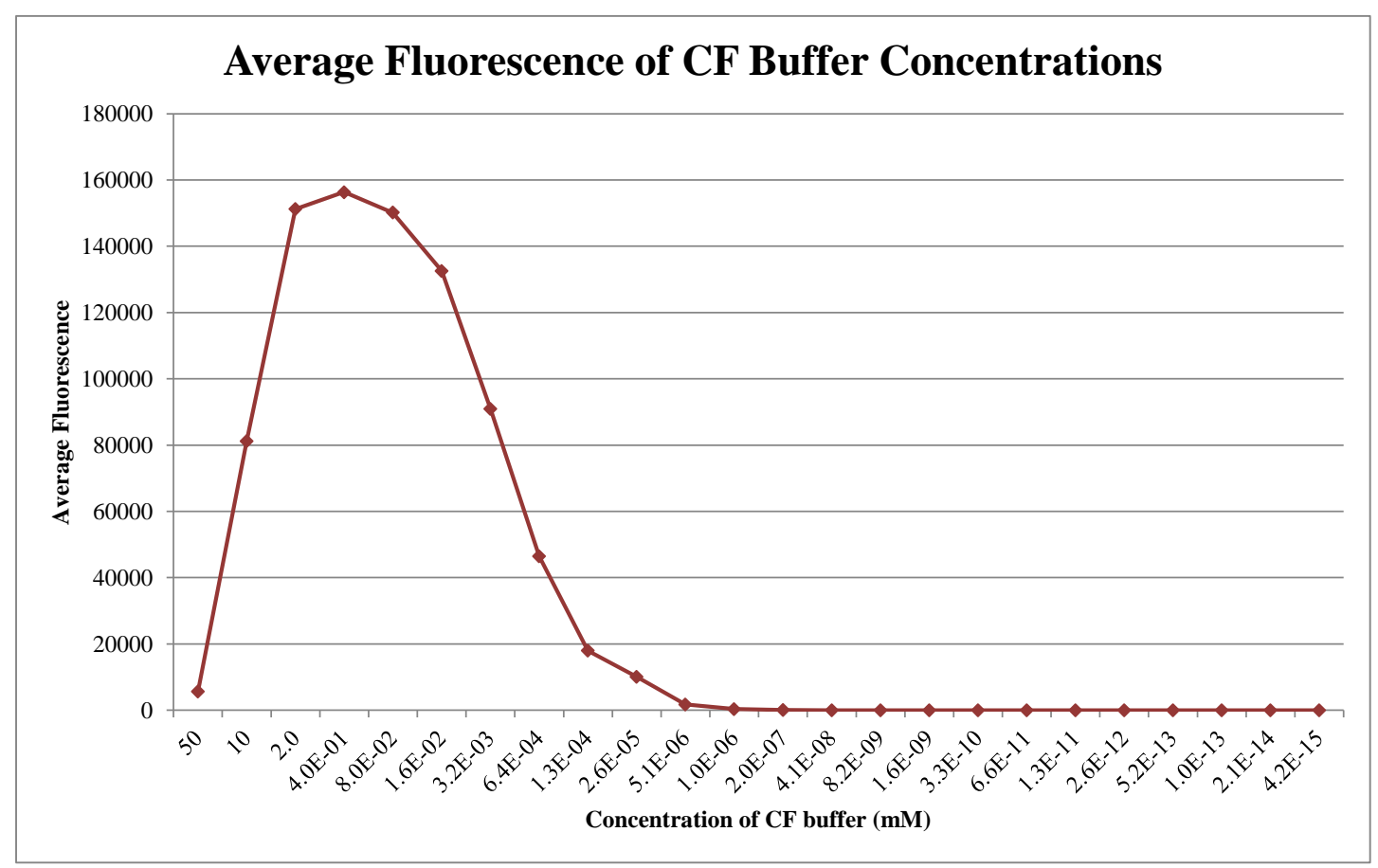

Figure 3.15. Carboxyfluorescein (CF) buffer dilution curve measuring excitation at $485 \mathrm{~nm}$ and emission at $535 \mathrm{~nm}$ and using 20 flashes.

The most important parameter that needed to be established for this assay was the ability to reproducibly create liposomes with MscL incorporated into them. This was tested by using controls that would later be used in the screening assays. The positive control (LPC, see Section 2.5 of Chapter 2) was used to model the opening of MscL, a negative solvent control was used to test the sensitivity of the liposomes to the solvent, a negative 

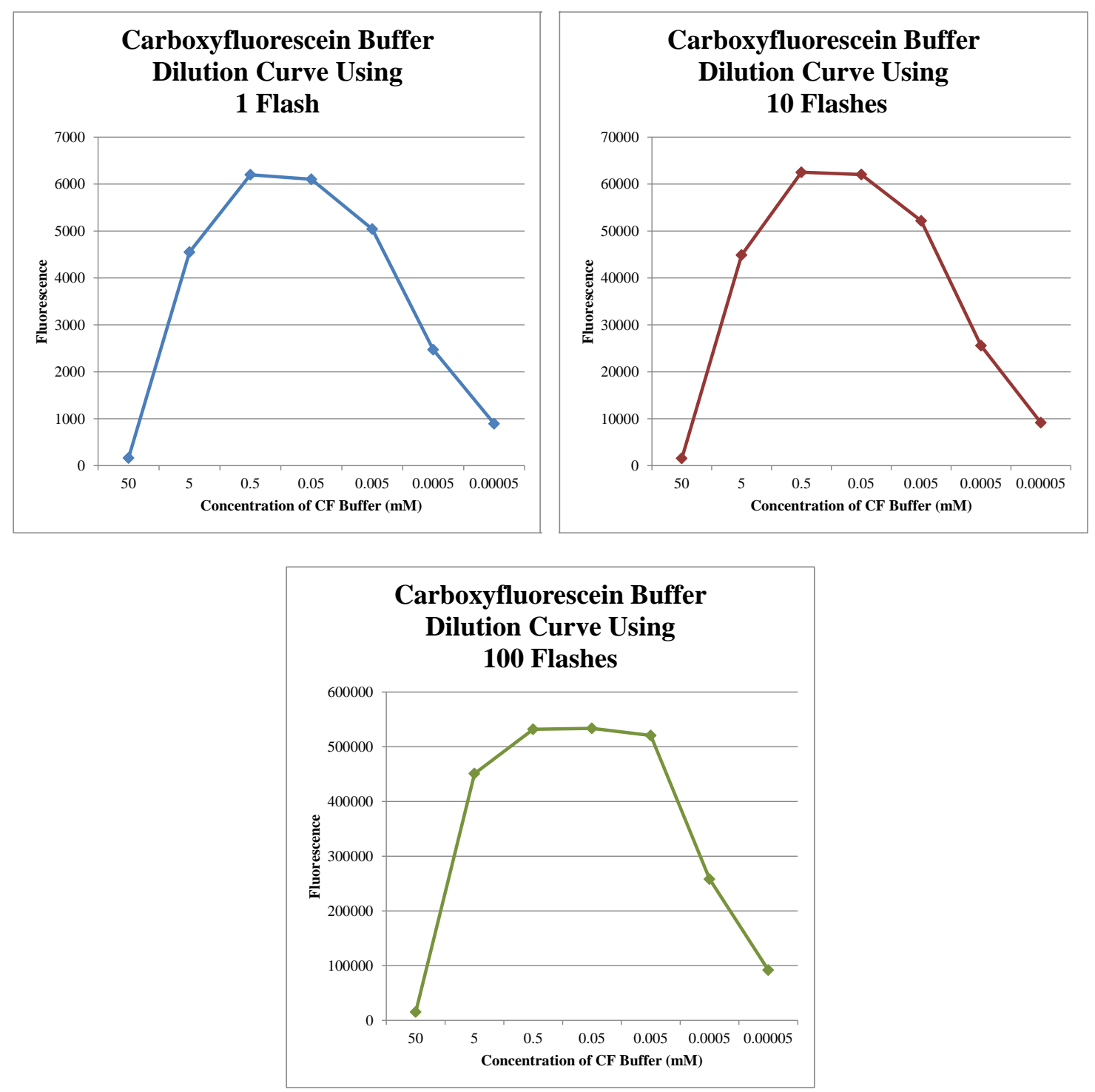

Figure 3.16. Carboxyfluorescein (CF) buffer dilution curve testing the effect of using 1 flash (top left, blue), 10 flashes (top right, maroon) and 100 flashes (bottom, green). Excitation was measured at $485 \mathrm{~nm}$ and emission at $535 \mathrm{~nm}$.

buffer control was used to determine the fluorescence of the liposomes in the absence of other additives and a Triton ${ }^{\mathrm{TM}} \mathrm{X}-100$ control was used to measure the total fluorescence of a burst liposome. All of these controls were used on both MscL-containing and control liposomes with all tested in triplicate where possible. This number of controls is necessary due to the fragility of these liposomes and their sensitivity to subtle variations in conditions. For example, the MscL liposomes were often found to be more fragile than the control liposomes and would start to leak CF over time, even in the solvent controls.

The fluorescence of these assays had to be read over an extended period of time due to the mode of action of the positive control LPC, which needed time to incorporate into the liposome membrane causing a distortion, thus opening the MscL. This meant that the LPC was the last control to be added to the plate and the fluorescence was then read in 5 or 10 minute intervals after the LPC addition over a period of 30-60 minutes. Reading these plates over time allowed the increase in fluorescence occurring in the 
MscL-containing liposomes that had been exposed to LPC to be seen and compared to the increase in fluorescence occurring in the negative control liposomes over time. Ideally, the MscL-containing liposomes would show the greatest increase in fluorescence when exposed to LPC as opposed to the negative controls. However, this was not always the case, the LPC effect on the MscL would sometimes show only a small increase in fluorescence, bringing into question whether the MscL had actually been incorporated into the liposomes and if it had, if the liposomes were too fragile to give reliable results. Another possible reason for this inconsistency may be the variable size of MscL channel clusters that have been shown to form upon MscL reconstitution into liposomes. Upon MscL channel activation these clusters separate into clusters of closed and clusters of open channels, with the number of channels in each of these clusters varying depending on the membrane tension activating the channels. ${ }^{103}$ This would mean that the number of MscL channels opened by the LPC would vary depending on the nature of the channel clusters occurring.

In testing the liposomes, both the control liposomes and MscL liposome concentrations were made up to achieve a similar fluorescence in the fluorometer, in both the intact and burst states. The fluorescence of the liposomes was analysed as a percentage of the total fluorescence possible (from the Triton ${ }^{\mathrm{TM}}$ control). This meant that the results could be compared between the MscL and control liposomes (as well as across assays) without exactly matching the concentration (and hence fluorescence) of the liposomes. The optimal fluorescence to aim for when diluting the liposomes, which would show the greatest difference in fluorescence between the closed and burst liposomes, had to be established to allow for maximal sensitivity in the assay.

Since the actual concentration of liposomes cannot be established in the solution recovered from the gel filtration, only concentrations relative to this solution can be reported. An experiment using this liposome solution alongside $\frac{1}{10}, \frac{1}{20}, \frac{1}{30}, \frac{1}{40}, \frac{1}{50}, \frac{1}{60}$ and $\frac{1}{70}$ dilutions was performed, testing both intact and burst liposomes (Table 3.1). This showed that the greatest differences in fluorescence values could be seen with increasing concentrations of liposomes, however, it is the percentage of the burst liposome fluorescence that is important for the intact liposomes. The lower this relative fluorescence, the easier to note the difference in relative fluorescence when MscL incorporated in the liposome is opened. From this experiment, it was proposed that liposome solutions with fluorescences of approximately 20000 in the closed state and 50000 in the open state would fit the requirements for this assay. This showed a reasonable difference in fluorescence with the closed liposomes having $38 \%$ the fluorescence of the burst liposomes. In this experiment such fluorescence intensities were achieved with a dilution of approximately $\frac{1}{40}$. It should be noted that the $\frac{1}{30}, \frac{1}{50}$ and $\frac{1}{60}$ dilutions would have been equally as effective and the $\frac{1}{40}$ dilution was chosen arbitrarily as it was a reasonable dilution. The $\frac{1}{70}$ dilution with $42 \%$ difference in fluorescence is an outlier of the data, this value is a good example of the inconsistencies encountered over the course of this work. 
Table 3.1. Comparison of fluorescence in control and burst CF-containing liposomes.

\begin{tabular}{ccccc}
\hline & \multicolumn{2}{c}{ Average Fluorescence } & & \\
\cline { 2 - 3 } Dilution & Control liposomes & Burst liposomes & Difference & Percentage (\%) \\
\hline 1 & 62,679 & 129,698 & 67,019 & 48 \\
$\frac{1}{10}$ & 40,497 & 94,578 & 54,081 & 43 \\
$\frac{1}{20}$ & 29,721 & 73,395 & 43,674 & 40 \\
$\frac{1}{30}$ & 23,219 & 60,042 & 36,823 & 39 \\
$\frac{1}{40}$ & 20,081 & 52,943 & 32,862 & 38 \\
$\frac{1}{50}$ & 18,504 & 47,915 & 29,411 & 39 \\
$\frac{1}{60}$ & 16,119 & 42,722 & 26,603 & 38 \\
$\frac{1}{70}$ & 16,302 & 38,679 & 22,377 & 42 \\
\hline
\end{tabular}

The relative fluorescence is not the only variable that needs to be taken into consideration when choosing the dilution of liposomes to use. The concentration of LPC and liposomes used have to be balanced for maximal LPC effect without causing lysis or leakage of the liposomes from too many LPC molecules inserting into the membrane. In order to test this, assays altering the liposome concentrations and LPC concentrations were performed. Also, a concentration of liposomes that show the maximal effect from active extracts while not being affected by the inactive extracts was desired. Assays testing control liposomes in varying concentration of crude extract were used to test this.

Before assays against crude extracts could be performed, a suitable solvent for adding the extracts that would not affect the liposomes needed to be identified. This was done by testing control liposomes with an equivalent of $2 \mu \mathrm{L}$ of methanol or DMSO to $98 \mu \mathrm{L}$ of liposome solution, or an equivalent of $4 \mu \mathrm{L}$ of a 1:1 methanol to water mixture or a 1:1 DMSO to water mixture to $96 \mu \mathrm{L}$ of liposome solution. The fluorescence of these wells were then compared to the fluorescence of control liposomes with an equivalent of $2 \mu \mathrm{L}$ of DR buffer added to $98 \mu \mathrm{L}$ of liposome solution. This experiment showed that pure methanol affected the liposomes the least and so methanol was used in subsequent experiments to test crude extracts (Figure 3.17).

A selection of crude extracts was tested against control liposomes to confirm that they would not affect control liposomes at the extract concentrations tested. Liposome solutions were tested with a maximum of $10 \mu \mathrm{g}$ of extract in $2 \mu \mathrm{L}$ of methanol added to the wells, making a $100 \mu \mathrm{L}$ volume (concentration of extract: $100 \mu \mathrm{g} / \mathrm{mL}$ ) with serial 


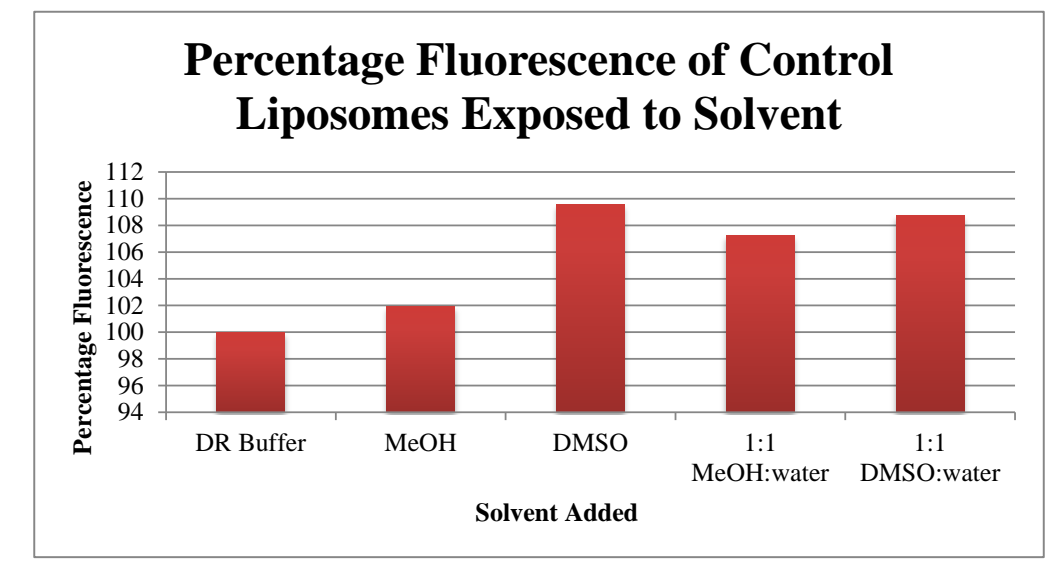

Figure 3.17. Control liposomes stability in different solvents as shown by their fluorescence relative to the DR buffer control.

dilutions used from there. The fluorescence of the liposomes was around 15000-20000 in the intact liposome solution. This experiment showed that the extracts chosen had no significant effect on the control liposomes with fluorescences that were between $93 \%$ and $110 \%$ the fluorescence of the solvent controls (Figure 3.18). This experiment also showed the variation that can be seen in fluorescence measurements between runs, with fluorescence readings of blank DR buffer control wells (without liposomes) ranging between 4 and 30 in one plate and between 14 and 53 in the other (data not shown). These variations in fluorescence were proposed to be insignificant at the level of fluorescence being measured in this assay.

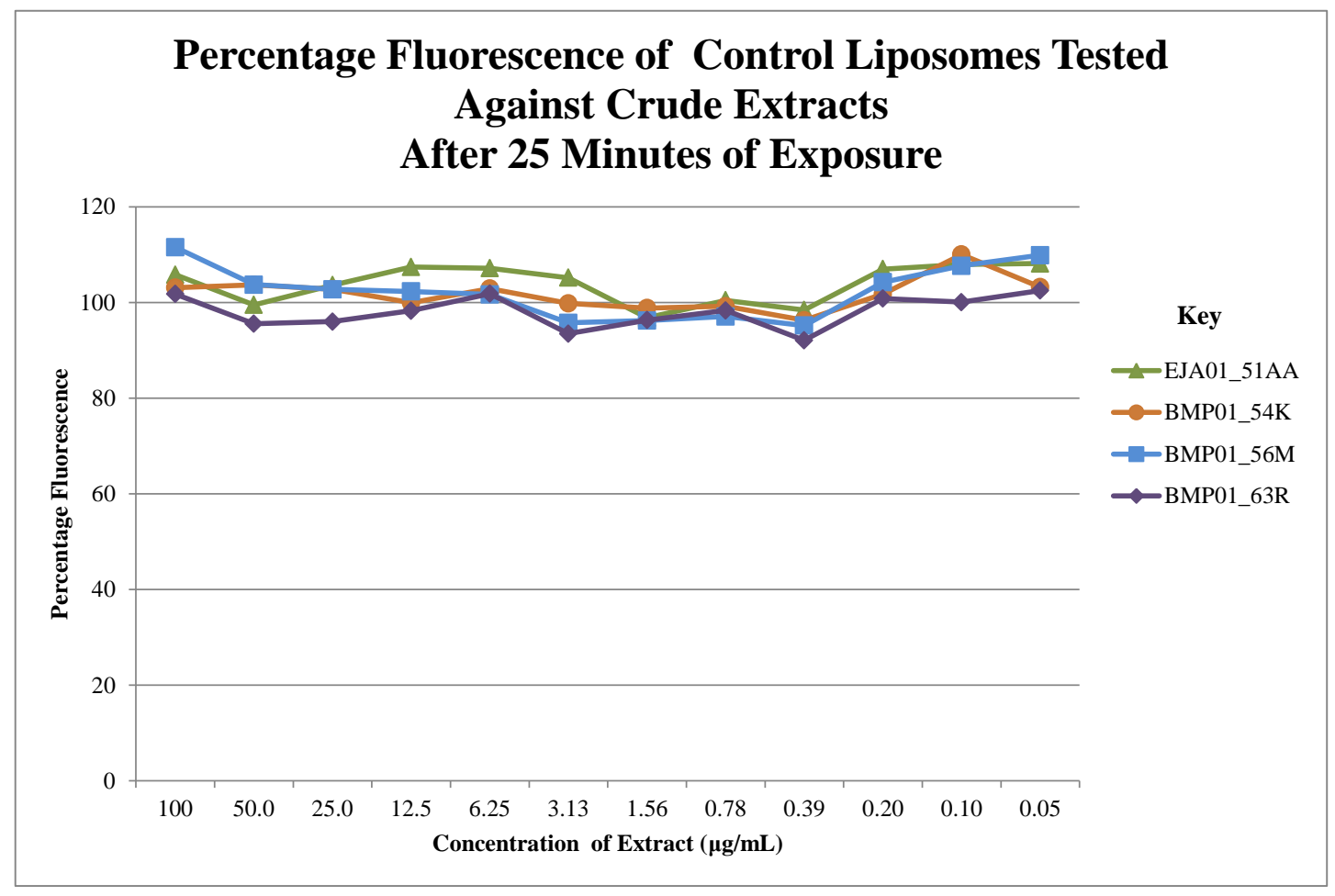

Figure 3.18. Control liposomes stability towards crude extracts, tested at various concentrations. All extracts tested at concentrations of $100 \mu \mathrm{g} / \mathrm{mL}$ and below show no significant effect on the control liposomes relative to the methanol (solvent) control. 
The LPC was used as a solution in methanol at a concentration of $50 \mathrm{mg} / \mathrm{mL}(0.1 \mathrm{M})$. When $2 \mu \mathrm{L}$ of this was added to $98 \mu \mathrm{L}$ of liposome solution (0.002 M LPC) the opening of MscL was able to be detected. However, this opening was detected by the increase in the percentage fluorescence of MscL-containing liposomes (relative to the burst liposomes fluorescence) compared with the control liposomes percentage fluorescence, when exposed to LPC. The maximum difference in the percentage fluorescence between the MscL and control liposomes would usually be around 6-8\% and so it was questioned whether this would be significant enough for MscL opening to be distinguished from the closed controls. The effect of LPC that was generally seen in these assays was the fluorescence percentage difference between the MscL and control liposomes increasing until a maximum at around 30 minutes after LPC addition. Following this, the fluorescence percentage difference would start to decrease as the LPC molecules equilibrated in the membrane causing the MscL to close again (see Section 2.5 of Chapter 2). The reason that the fluorescence percentage difference started to decrease was that the MscL were no longer open and releasing the $\mathrm{CF}$ molecules but the control and MscL-containing liposomes were both still leaking CF molecules due to their poor stability. LPC molecules were also able to cause leaking in liposomes if enough of them inserted into the membrane to cause a disruption, this resulted in a steady increase of the percentage fluorescence of both MscL and control liposomes occurring until lysis of the liposomes. This leakage caused by LPC resulted in fluctuations in the percentage fluorescence difference between the control and MscL liposomes.

Experiments were done adjusting the concentration of LPC in order to see if a lower concentration would cause a more distinctive effect on the MscL-containing liposomes when compared to controls. It was found that when $0.5 \mu \mathrm{L}$ of $0.1 \mathrm{M} \mathrm{LPC}$ was added to MscL liposomes, the effect of the increased percentage of fluorescence was more distinct than in the liposomes that 1 and $2 \mu \mathrm{L}$ of LPC had been added to. This can be seen in Figure 3.19, which shows the percentage fluorescence (relative to the Triton ${ }^{\mathrm{TM}}$ control) of the control and MscL liposomes when exposed to varying concentrations of LPC. The control and MscL liposomes exposed to DR buffer and methanol are also shown on this graph in order to demonstrate the magnitude of the fluorescence difference. The graph shows that the $0.5 \mu \mathrm{L}$ addition of LPC to control liposomes (shown by the green squares) affects the fluorescence the least and this in turn makes the effect on the MscL liposomes (shown by green circles) more significant when calculating the difference in these percentage fluorescences. 


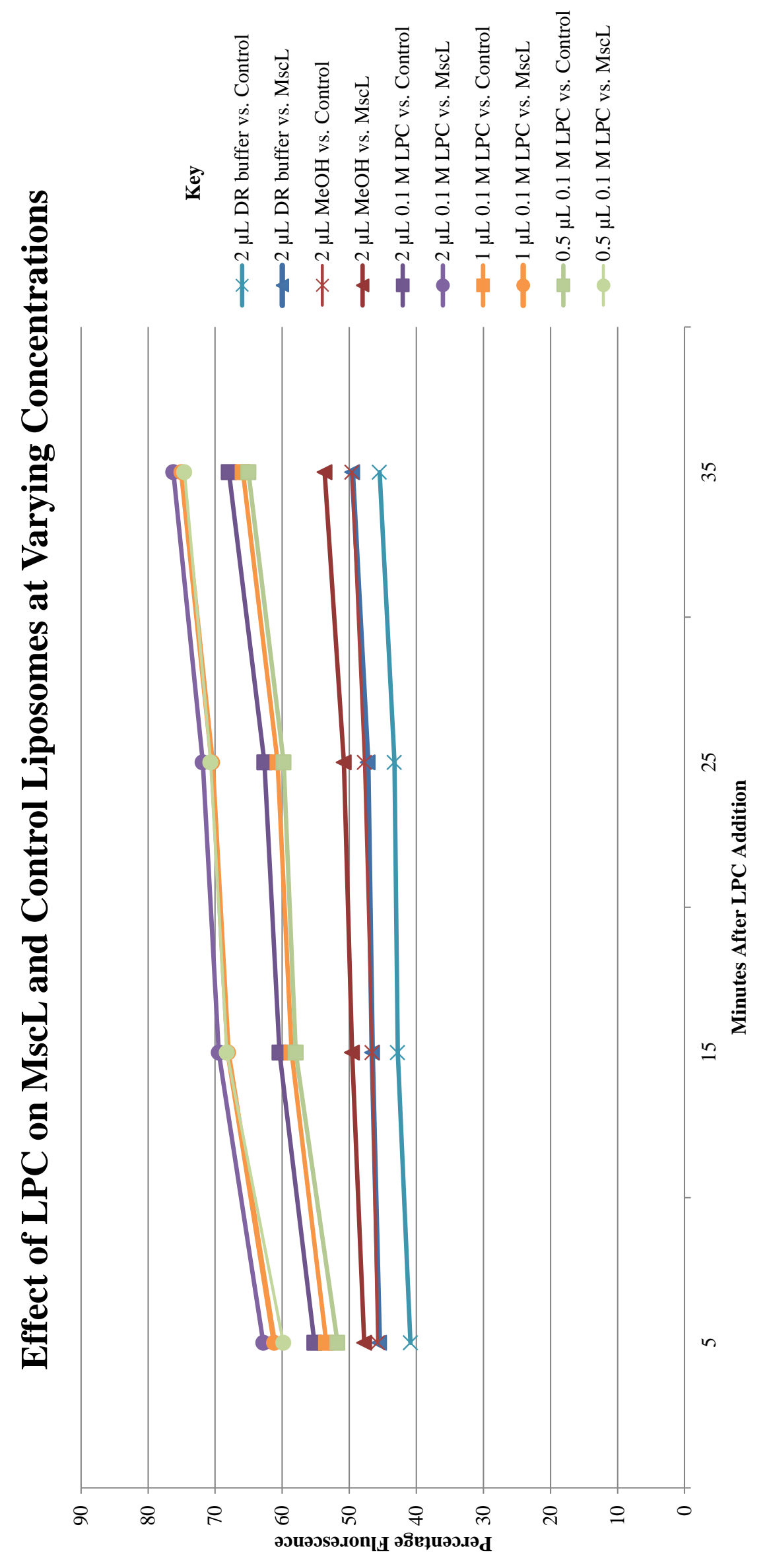

Figure 3.19. Fluorescence (relative to completely burst liposomes) of MscL and control liposomes when exposed to various concentrations of LPC. 
This led to the LPC solution being diluted to a $0.05 \mathrm{M}$ concentration $(25 \mathrm{mg} / \mathrm{mL})$ and tested again at $0.5,1$ and $2 \mu \mathrm{L}$ additions. The liposome stability was poor in these experiments, which meant that the LPC caused all of the liposomes to leak and eventually burst. Generally, the effect of poor stability is much clearer in the MscL-containing liposomes as the presence of MscL in the membranes appears to make them weaker (possibly an indicator that lower amounts of the MscL protein should be incorporated in the liposomes), though both the control and MscL-containing liposomes were affected by poor stability in this assay. This meant that the results obtained from this assay were not conclusive. However, it was decided to proceed using $0.5 \mu \mathrm{L}$ additions of $0.05 \mathrm{M}$ LPC as the positive control in assays in the interest of time. This volume was chosen as it was hoped to have minimal effect on the control liposomes making the LPC effect even more significant.

In the same assay determining the concentrations of LPC to be used, the concentrations of liposomes were tested to determine the optimal combination. Liposome concentrations that exhibited fluorescence at approximately 25000-26000, 15000-16000 and 900010000 in the closed state were used and tested using all of the controls, including the three different LPC addition volumes. Because of the poor stability in this assay it was not clear which of the three concentrations tested was optimal. However, it was clear that all three concentrations were acceptable as they all showed clear differences in fluorescence between the MscL and control liposomes (making the erratic differences in this assay very clear). The time taken to set up this assay was longer than usual and so the plates were only able to be read at 20,30, 40 and 55 minutes after LPC addition. Despite the erratic values, it could be seen that the fluorescence percentage difference increased between 20 and 30 minutes for the liposomes at a fluorescence of 25000 and following 30 minutes this difference began to decrease. For the liposomes at a fluorescence of 15000 and 9000, the fluorescence percentage differences just decreased from the first reading. This decrease in the fluorescence difference between the control and MscL liposomes can be seen in Figure 3.20 by the MscL and control liposome fluorescences at the same concentration trending towards convergence at $100 \%$ fluorescence. This was thought to indicate that the effect of LPC had been much faster in the lower concentration of liposomes compared with the higher concentration.

Since a faster LPC effect would mean that the assay plates could be read for a shorter time period (which is especially useful because of the short time frame of viability for these liposomes), it was decided to use the lower concentration of liposomes at a fluorescence of 9000-10000. Additionally, a lower concentration of liposomes would hopefully mean that they are more sensitive to compounds in the extracts being tested, however, this could also mean the liposomes are more sensitive to LPC. 


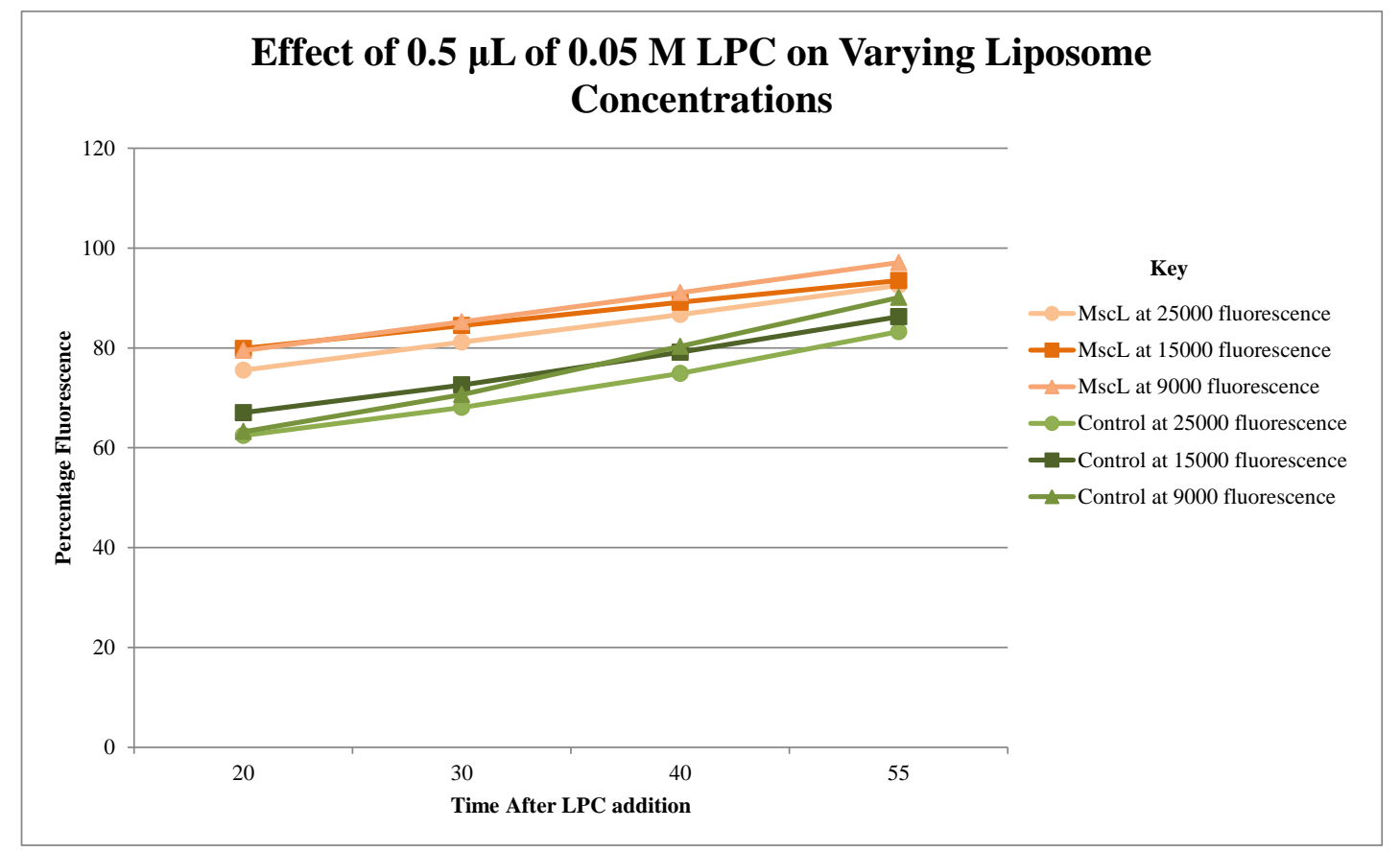

Figure 3.20. Effect of liposome concentration on the fluorescence change (relative to completely burst liposomes) caused by a $0.5 \mu \mathrm{L}$ addition of $0.05 \mathrm{M} \mathrm{LPC}$.

From this work, parameters to be used in this assay could be chosen. The CF dilution curve helped to establish that 20 flashes should be used on the fluorometer when reading the assay plates. The assay testing different dilutions of liposomes established that liposomes with a fluorescence of approximately 20000 in the closed state would be a good starting point for use in this assay with the intact liposomes displaying $38 \%$ of the fluorescence of the burst liposomes. The assay testing control liposomes against different solvents helped to establish that methanol had the smallest effect on these liposomes and so would be a good solvent to use in screening assays. The assay testing crude extracts against control liposomes showed that at a concentration of $100 \mu \mathrm{g} / \mathrm{mL}$ these crude extracts had no significant effect on these liposomes. This established that $100 \mu \mathrm{g} / \mathrm{mL}$ was a reasonable concentration to start the extract testing at. The LPC test assays established that smaller amounts of LPC showed a more significant effect as a lower concentration of LPC molecules will reduce their effect on the control liposomes. This suggested that $0.05 \mu \mathrm{L}$ of a $0.05 \mathrm{M}$ LPC solution would be a reasonable amount to use for a positive control in a screening assay (resulting in a $25 \mu \mathrm{M}$ concentration of LPC when made up to $100 \mu \mathrm{L}$ ). This assay to test the LPC concentration to be used also showed that, while liposome concentrations resulting in fluorescences around 25000, 15000 and 9000 do not create obvious differences in the effectiveness of the assay, using a smaller concentration of liposomes may make the effects of LPC and active extracts appear faster, shortening the time that the plate must be read over. This would be desirable as it would enable more experiments to be run on one batch of liposomes, therefore a lower concentration of liposomes resulting in a fluorescence of approximately 10000 was proposed to be used. 


\subsubsection{Testing the Assay}

Despite the inconsistencies that were observed in some of the experiments due to instability of the liposomes, several key parameters to be used in the MscL assay were chosen. These parameters were used when attempting to test the extract library of New Zealand and Tongan marine invertebrates for activity towards MscL. Unfortunately, the fragility of the liposomes gave erratic results, leading to no 'hits' being identified. It is possible that no 'hits' were identified due to the highly specific nature of the assay. Probing such a specific mode of action, the likelihood of no Msc-active compounds being present in the extracts tested is high when compared to assays with multiple targets. Despite this possibility, the instability and poor reproducibility of the liposomes used in this assay was a concern as it is an indicator of fragile liposomes. One of the issues that may have lead to the fragile liposomes was the HEPES buffer (that makes up one tenth of DR buffer, which is used as the medium for this assay) not being changed often enough. The growth of microorganisms in the HEPES buffer could have caused contamination in the DR buffer medium. This is likely to have contributed to some of the inconsistencies observed, however, it is not thought to be the sole cause. Even when fresh HEPES buffer was made up and used, the assay gave fragile liposomes and the effect of LPC on the MscL-containing liposomes was still weak.

There are more parameters that need to be optimised for this assay before it is able to be used to test extract libraries for Msc-modulating compounds. For example, the amount of MscL that is added to the liposomes to be incorporated into the membrane needs to be adjusted in order to find the optimal level of incorporation. Also, the MscL proteins are suspended in detergent, this means that during the incorporation procedure BioBeads ${ }^{\circledR}$ are used to remove the excess detergent that would otherwise make the liposomes more fragile. The maximum time that the BioBeads ${ }^{\circledR}$ were left mixing with the liposome solution in this work was four hours, adjusting this time (most likely by making it longer) could increase the stability observed in the liposomes.

One very important issue with this assay is that it takes most of a day to prepare the liposomes so that the experiment can only be run at the end of the day when the liposome preparation is complete. The experiment also has to be run right after liposome preparation is complete because the fragile liposomes will burst over time and by the next day the liposome solution will no longer be viable. If protocols could be modified in running this assay, allowing the majority of preparation time to be done the day before an assay is run, this would increase the usefulness and would allow more experiments to be run with each batch of liposomes. One way that this may be possible is by leaving the liposomes mixing with the BioBeads ${ }^{\circledR}$ overnight and separating the liposomes from the unincorporated $\mathrm{CF}$ the next day.

Time did not permit further work on this assay meaning that it has not been explored 
enough to obtain reliable results in the screening of an extract library. Further work on this assay will need to be done in order to better understand all the variables that affect liposome preparation and therefore stability. If this can be better understood then liposomes that are robust enough to provide consistent and reproducible results and sensitive enough for a clear distinction to be seen between negative controls and a positive 'hit' can hopefully be produced, allowing for this assay to be used reliably in the identification of bioactive natural products as molecular probes for Msc. 


\section{Chapter 4}

\section{3-Alkyl Pyridinium Alkaloids}

\subsection{Introduction}

3-Alkyl pyridinium alkaloids (3-APAs) are potent bioactive compounds that have been found from sponges of the order Haplosclerida (Figure 4.1). The first 3-APAs to be isolated and identified were a mixture of polymeric 3-APAs reported in 1978 by Schmitz et al. called halitoxins. ${ }^{104} 3$-APAs can be cyclic or linear and can vary in chain length, unsaturation of the alkyl chain, branching and number of pyridinium units. Linear 3-APAs can also vary in the terminal functional groups of the alkyl chain. No naming system has been set out for the 3-APAs and this has resulted in some structurally similar compounds being given very different names and some compounds not being named yet. This highlights the need for a clear naming system to be set out for these compounds. This introductory review is limited to isolated cyclic compounds of natural origin that contain the 3-alkyl pyridinium moiety (15) and does not include polymeric structures or 3-alkyl pyridines. The 3-APAs reported here are limited to six or less monomeric units. The compounds that meet these criteria are reported in Tables 4.1 to 4.4. For a comprehensive review on 3-alkyl pyridine and pyridinium structures that have been identified prior to 2008, including the polymeric halitoxins and amphitoxins, see Turk et al. (2008). ${ }^{105}$<smiles>CCc1ccc[n+](C(C)(C)C)c1</smiles>

15 
Phylum: Porifera

Class: Demospongiae

Subclass: Ceractinomorpha

Order: Haplosclerida

Family: Phloeodictyidae

Genus: Calyx

Family: Niphatidae

Genus: Niphates

Pachychalina

Amphimedon

Family: Chalinidae

Genus: Haliclona

Reniera

Family: Callyspongiidae

Genus: Callyspongia

Family: Petrosiidae

Genus: Xestospongia

Figure 4.1. Taxonomic classification to genus level of Haplosclerid sponges that 3-APAs have been isolated from. ${ }^{18,106}$

\subsection{Linear 3-APAs}

The linear 3-APAs that have been reported in the literature to date fall into four general structural categories: the xestamines, niphatoxins, viscosalines and pachychalines, each one being distinguished by the terminal functional group. The xestamines are usually isolated as 3-alkyl pyridines, however, three $\mathrm{N}$-methyl pyridinium salts of these have been isolated. The xestamines have methoxy(methyl)amine terminal groups and have been isolated with both saturated and unsaturated alkyl chains (including a conjugated enyne in xestamine F, 16). These compounds inhibit the growth of Staphylococcus aureus, Bacillus subtilis and Candida albicans. ${ }^{107}$ There are three known niphatoxins and these are characterised by terminal pyridines. The niphatoxins that have been isolated all have one pyridinium unit and unsaturation in the alkyl chain (e.g. niphatoxin A, 17). The niphatoxins $\mathrm{A}$ and $\mathrm{B}$ have shown ichthyotoxic and cytotoxic activity against P388 (mouse leukaemia) cells and niphotoxin $\mathrm{C}$ has shown cytotoxic activity against THP-1 (human leukemic monocyte) cells. ${ }^{108,109}$ 
There have been five different viscosalines reported in the literature to date and these have a pyridine group at the terminus of the $\mathrm{N}$-alkyl chain of the pyridinium and a $\beta$-alanine group at the terminus of the $\mathrm{C}$-alkyl chain of the pyridinium (e.g. viscosaline $\mathrm{B}_{1}, \mathbf{1 8}$ ). The viscosalines that have been fully characterised all differ in the length of the saturated alkyl chains. ${ }^{110,111}$ However, an unsaturated viscosaline has been found by Schmidt et al. that has not yet been fully characterised. ${ }^{111}$ The first viscosaline to be isolated inhibited the growth of several different microorganisms in disk diffusion assays. ${ }^{110}$

There are seven different pachychalines that have been reported, they are characterised by terminal amino groups or one terminal amino group and one norspermidine (e.g. pachychaline B, 19). The pachychalines that have been reported have from one to three pyridiniums (monomer to trimer) and vary in the alkyl chain lengths and saturation or unsaturation of alkyl chains, showing the most variation among the linear 3-APAs reviewed here. The pachychalines are also very unusual as the dimer and two of the trimer pyridiniums are connected by an alkyl chain that links the 3 position of both pyridiniums. This creates an unusual carbon-alkyl-carbon motif, which can also be seen in the niphatoxins, rather than the usual nitrogen-alkyl-carbon motif. The pachychalines A-C showed activity against the cell lines MDA-MB-231 (human breast adenocarcinoma), A549 (human lung carcinoma) and HT29 (human colon adenocarcinoma). ${ }^{112}$

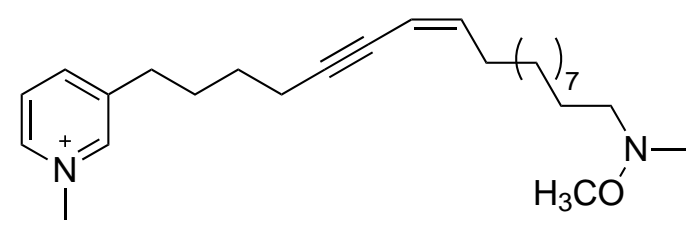

16

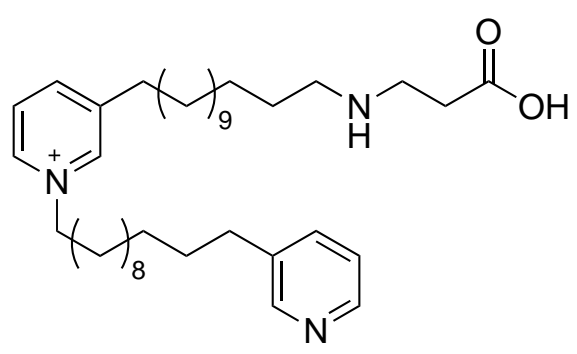

18

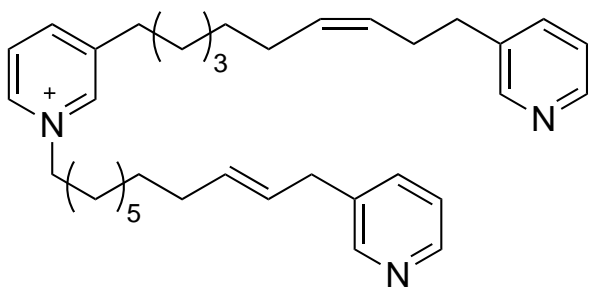

17

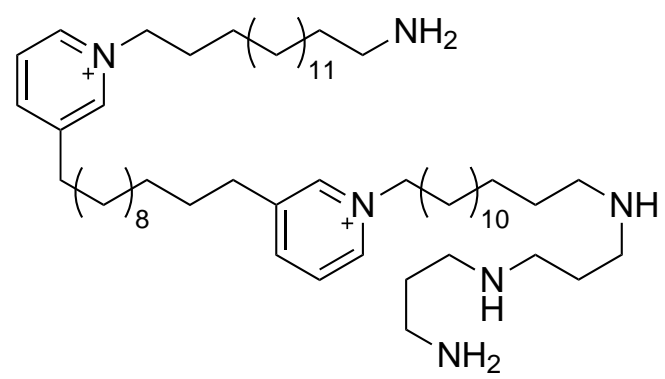

19

\subsection{Cyclic 3-APAs}

\subsubsection{Monomers}

In 1996, the first cyclic 3-APA monomer to be identified, haliclocyclin C (20) was synthesised in order to confirm the dimeric structure of cyclostellettamine C (see Section 
4.3.2). In this study, haliclocyclin $C$ showed antagonistic activity toward the muscarinic receptors of rat brain, heart and salivary gland with mean $\mathrm{IC}_{50}$ values of 1.7, 2.8 and $2.9 \mu \mathrm{g} / \mathrm{mL}$, respectively. In this assay the monomeric haliclocyclin $\mathrm{C}$ was less active than the dimeric cyclostellettamine $\mathrm{C}$, which had mean $\mathrm{IC}_{50}$ values of 90,89 and $195 \mathrm{ng} / \mathrm{mL}$, respectively. ${ }^{113}$ Haliclocyclin C was then accidentally synthesised in 1998 by Kaiser et al. when devising a new, selective strategy for synthesising 3-APA dimers and oligomers. ${ }^{114}$

A cyclic 3-APA monomer was not found naturally until 2008 when Köck and coworkers reported the isolation of haliclocyclin F (21) from the Arctic marine sponge Haliclona viscosa. This compound was only isolated in a small amount so it was also synthesised for biological testing to be performed. Haliclocyclin F was tested against E. coli tolC, S. aureus and L929 mouse fibroblast cells along with cyclostellettamine C, viscosamine (see section 4.3.3) and the synthesised haliclocyclin C. Of the compounds tested in these assays, haliclocyclin $\mathrm{F}$ was the most active with an $\mathrm{IC}_{50}$ of $0.3 \mu \mathrm{g} / \mathrm{mL}$ against mice fibroblasts (showing cytotoxicity) and growth inhibition diameters of 23 and $22 \mathrm{~mm}$ against $E$. coli tolC and $S$. aureus, respectively, when sample loadings of $20 \mu \mathrm{g}$ were used. ${ }^{115}$ This study also reported that in molar concentrations haliclocyclin $\mathrm{C}$ is less cytotoxic than cyclostellettamine $\mathrm{C}$ and viscosamine but haliclocyclin $\mathrm{C}$ also had a larger zone of inhibition in the antibacterial assays than the larger compounds. ${ }^{115}$ In 2011, Köck and co-workers finally isolated haliclocyclin $\mathrm{C}$ from $H$. viscosa. This was the first time this compound had been isolated from a natural source. In this report they proposed the naming system for the 3-APA monomers and named the haliclocyclins $\mathrm{C}$ and $\mathrm{F}$ after the corresponding cyclostellettamines $\mathrm{C}$ and $\mathrm{F}$ that also contained $\mathrm{C}_{13}$ and $\mathrm{C}_{14}$ alkyl chains, respectively. ${ }^{116}$

Table 4.1. Cyclic Monomer 3-Alkyl Pyridinium Alkaloids.

Reported Activity* Reference

$* \mathrm{~B}=$ Antibacterial, $\mathrm{N}=$ Neurotoxin, $\mathrm{T}=$ General Toxicity 


\subsubsection{Dimers}

In 1994, the cyclostellettamines A-F (22-27) were first reported to have been isolated from the marine sponge Stelleta maxima by Fusetani et al. ${ }^{117}$ Upon re-examination of the sponge, Fusetani and co-workers concluded that the cyclostellettamines had, in fact, been isolated from an epiphitic Haliclona sp. in the sample. ${ }^{118}$ The cyclostellettamines A-F contain two pyridinium units connected by two saturated alkyl chains with 12 to 14 carbon units. They showed potent antagonistic activity toward the muscarinic receptors of rat brains, hearts and salivary glands with $\mathrm{IC}_{50}$ values in the range of 0.03 to $0.47 \mu \mathrm{g} / \mathrm{mL} .{ }^{117}$ The next cyclostellettamines to be isolated were isolated by the same group (Fusetani and co-workers) in 2004 from the marine sponge Xestospongia sp. The group re-isolated cyclostellettamine A as well as isolating one new saturated 3-APA dimer cyclostellettamine G (28) and the two dehydrocyclostellettamines D (29) and E (30), which contain a $Z$-geometry alkene in one of the alkyl chains. ${ }^{118}$ These compounds were tested for inhibitory activity towards the histone deacetylase enzyme (HDAC), derived from K562 human leukemia cells, as well as for cytotoxic activity against HeLa (human cervix carcinoma), P388 (mouse leukemia) and 3 Y1 (rat fibroblastic) cell lines. The $\mathrm{IC}_{50}$ values against HDAC were in the range of 17 to $80 \mu \mathrm{M}$. The cytotoxicity against the cancer cell lines P388 and HeLa had $\mathrm{IC}_{50}$ values in the range of 0.6 to $2.8 \mu \mathrm{M}$ and the cytotoxicity against the $3 \mathrm{Y} 1$ fibroblasts had $\mathrm{IC}_{50}$ values in the range of 3.2 to $11 \mu \mathrm{M}{ }^{118}$

De Oliveira et al. isolated more cyclostellettamines, also in 2004. From the marine sponge Pachychalina sp. the known cyclostellettamines A-G (22-28) were isolated along with the new cyclostellettamines H, I, K and L (31-34). These new cyclostellettamines were very similar to those previously identified with the differences being the number of methylene units in the alkyl chain, which ranged from 10 to 14 units. The name cyclostellettamine $\mathrm{J}$ was reserved for a cyclostellettamine with $\mathrm{C}_{11}$ and $\mathrm{C}_{13}$ carbon chains that has not yet been reported but would be expected to exist given the pattern of the cyclostellettamines isolated to date. ${ }^{119}$ The cyclostellettamines A-F (22- 27) isolated from Pachychalina sp. and the synthesised cyclostellettamines G-I (28-32), K (33) and $\mathrm{L}$ (34) were subjected to biological testing in antimicrobial assays. The compounds were tested against $S$. aureus (ATCC 25923), E. coli (ATCC 25922), Pseudomonas aeruginosa (ATCC 27853), Oxacillin-resistant $S$. aureus strain 8, Oxacillin-resistant $S$. aureus strain 108, P. aeruginosa 13 (which produces biofilms and is resistant to some antibiotics), P. aeruginosa $\mathrm{P} 1$ (also produces a biofilm and is resistant to some antibiotics), C. albicans (ATCC 10231) and Mycobacterium tuberculosis H37Rv. However, cyclostellettamine A and $\mathrm{B}$ were not tested against $C$. albicans as there was insufficient mass of these compounds isolated. Cyclostellettamine A, overall, appeared to be the least active as an MIC of no lower than $32 \mu \mathrm{g} / \mathrm{mL}$ was found in any of the assays performed and so will be excluded from the following discussion of activity. The most activity was seen against $S$. aureus with MIC values ranging from 0.3 to $2.4 \mu \mathrm{g} / \mathrm{mL}$. The compounds also displayed 
good activity against $E$. coli with MIC values ranging from 1.2 to $9.5 \mu \mathrm{g} / \mathrm{mL}$ (excluding cyclostellettamine $\mathrm{G}$, which gave an MIC of $39.1 \mu \mathrm{g} / \mathrm{mL}$ ). Against $M$. tuberculosis, MIC values in the range of $4.0-11.0 \mu \mathrm{g} / \mathrm{mL}$ were observed. In the $C$. albicans assay only cyclostellettamines $\mathrm{C}-\mathrm{F}$ showed any activity with MIC values of 0.12 to $0.7 \mu \mathrm{g} / \mathrm{mL}$. In all the other assays the activities observed ranged from poor to good with the activity apparently being highly dependant on the length of the alkyl chains. ${ }^{120}$

In 2005, Ondeyka et al. isolated a new dehydrocyclostellettamine with the two pyridinium rings connected by two $\mathrm{C}_{10}$ alkyl chains, one of which contained an $E$-alkene (35). ${ }^{121}$ This new 3-APA was not named by the authors. Compound 35 was an antagonist of the CXCR3 receptor that is expressed during certain inflammatory processes, with an $\mathrm{IC}_{50}$ of $0.69 \mu \mathrm{M}$, meaning it could play a role in modulation of the immune system. This could make it a useful drug in treatments relating to organ transplantation and diseases such as multiple sclerosis. ${ }^{121}$ In 2006, Teruya et al. identified cyclohaliclonamine A (36) as part of a mixture of dimeric, trimeric, tetrameric, pentameric and hexameric 3-APAs isolated from a marine sponge Haliclona sp. This compound had an $E$-alkene in each of the $\mathrm{C}_{10}$ alkyl chains connecting the two pyridinium units. ${ }^{122}$ In 2007, Xu et al. then isolated a similar E-geometry, unsaturated 3-APA with two $\mathrm{C}_{11}$ alkyl chains, 8,8'-diene-cyclostellettamine (37) from the marine sponge Amphimedon compressa. This compound differed from cyclohaliclonamine A not only in the alkyl chain lengths but also in the different position of the alkene functionalities, which were only one methylene away from the 3-position on the pyridiniums rather than two methylenes away from the nitrogen. ${ }^{123}$ 8,8'-diene-cyclostellettamine was a potent antifungal and antibacterial with $\mathrm{IC}_{50}$ values in the range of $0.25-2.5 \mu \mathrm{g} / \mathrm{mL}$ against C. albicans, E. coli, Pseudomonas aeruginosa, Cryptococcus neoformans, MRSA and Aspergillus fumigatus. ${ }^{123}$ An isomer of 8,8'-diene-cyclostellettamine was isolated in 2009 by Casapullo et al. from the sponge Haliclona sp. (38), this structure only differed from 37 in the geometry of the alkene groups, which were both Z. ${ }^{124}$ Compound $\mathbf{3 8}$ showed weak cytotoxicity with $\mathrm{IC}_{50}$ values greater than $20 \mu \mathrm{g} / \mathrm{mL}$ in assays against J774.A1 (murine macrophage), HEK293 (human embryonic kidney), and WEHI-164 (murine fibrosarcoma) cell lines. ${ }^{124}$

The first cyclic 3-APAs with branched chains were isolated by Laville et al. in 2009 from the sponge Reniera sp. along with an isomer of cyclohaliclonamine A. Njaoaminium A (39) has Z-alkenes at the C-13 to C-14 and C-13' to C-14' positions whereas cyclohaliclonamine A (36) has E-alkenes at the C-9 to C-10 and C-9' to C-10' positions. Njaoaminium B (40) has the same basic structure as njaoaminium A (39) with the addition of two $R$-configuration methyl groups at the $\mathrm{C}-8$ and $\mathrm{C}-8$ ' positions. Njaoaminium $\mathrm{C}$ (41) is similar to both njaoaminium A and B with the exception of only one $R$-configuration methyl group being present at the C-8 position. ${ }^{125}$ The njaoaminiums were tested against A549 (lung carcinoma), HT29 (colon adenocarcinoma) and MDA-MB-231 (breast adenocarcinoma) human cell lines. Njaoaminium A and C did 
not show cytotoxicity at concentrations below $10 \mu \mathrm{M}$ and so were considered to have poor activity. Njaoaminium $\mathrm{B}$ had moderate activity with $\mathrm{IC}_{50}$ values of $4.1,4.2$ and $4.8 \mu \mathrm{M}$ against the lung, colon and breast tumour cell lines, respectively. ${ }^{125}$

In 2012, Lee et al. isolated 10 new cyclostellettamines from the sponge Haliclona sp. They reported two of these compounds, cyclostellettamines $\mathrm{N}(\mathbf{4 2})$ and $\mathrm{Q}(\mathbf{4 3})$, as known compounds as they had previously been synthesised, however, this was the first report of these compounds being isolated from natural sources. ${ }^{126}$ Cyclostellettamine $\mathrm{Q}$ was synthesised by Grube et al. in 2006 in order to improve understanding of these compounds and cyclostellettamines N and Q were synthesised by Schmidt et al. in 2009 in order to confirm the structure of haliclamines (tetrahydropyridinium equivalents of the cyclostellettamines). ${ }^{127,128}$ Cyclostellettamines $\mathrm{N}$ and $\mathrm{Q}$ contain $\mathrm{C}_{9} / \mathrm{C}_{10}$ and $\mathrm{C}_{10} / \mathrm{C}_{11}$ saturated alkyl chains, respectively. Two new cyclostellettamines with $\mathrm{C}_{8} / \mathrm{C}_{10}(\mathbf{4 4})$ and $\mathrm{C}_{10} / \mathrm{C}_{10}$ (45) saturated alkyl chains were also isolated. ${ }^{126}$ None of the new compounds reported in this paper were named. Compounds 46-51 were dehydrocyclostellettamines that varied in chain length and alkene position and geometry. ${ }^{126}$ These compounds were tested for cytotoxic activity toward A549 (lung cancer) cell lines, as well as for inhibitory activity towards the growth of $S$. aureus (ATCC 6538p), B. subtilis (ATCC 6633), Micrococcus luteus (IFO 12708), Salmonella typhimurium (ATCC 14028), Proteus vulgaris (ATCC 3851), E. coli (ATCC 35270), pathogenic fungal strains and the microbial enzymes isocitrate lyase, sortase $\mathrm{A}$ and $\mathrm{Na}^{+} / \mathrm{K}^{+}$-ATPase. With $\mathrm{IC}_{50}$ values greater than $100 \mu \mathrm{g} / \mathrm{mL}$ and $100 \mu \mathrm{M}$ against the pathogenic fungi, E. coli and microbial enzymes, these compounds were concluded to not be active in these assays. ${ }^{126}$ MIC values of 12.5 to $50 \mu \mathrm{g} / \mathrm{mL}$ were observed against $S$. aureus, 3.125 to $100 \mu \mathrm{g} / \mathrm{mL}$ were observed against $M$. luteus, 25 to $100 \mu \mathrm{g} / \mathrm{mL}$ were observed against $B$. subtilis, S. typhimurium and $P$. vulgaris. Against the lung cancer cell lines, all the compounds tested proved to be moderately cytotoxic, with $\mathrm{IC}_{50}$ values in the range of 14.7 to $28.9 \mu \mathrm{M}$ for all of the compounds except 48 , which appeared to be poorly cytotoxic with an $\mathrm{IC}_{50}$ of $89.4 \mu \mathrm{M}{ }^{126}$

\subsubsection{Trimers}

The first naturally occurring trimeric 3-APA was isolated from the sponge $H$. viscosa by Volk and Köck in 2003. This trimeric 3-APA was named viscosamine (52) and was isolated as a mixture with cyclostellettamine C. Viscosamine has three saturated $\mathrm{C}_{13}$ alkyl chains and so is the trimeric 3-APA equivalent of cyclostellettamine C. ${ }^{129}$ Viscosamine was synthesised and tested for antiprotozoal activity as well as cytotoxicity in 2011 by Rodenko et al. ${ }^{130}$ The antiprotozoal assays were performed against Trypanosoma brucei (the blood stream form), the drug resistant T. brucei $4427 \Delta$ TbAt1 and T. brucei $\mathrm{s} 427$ clone B48, Leishmania major promastigotes, Leishmania mexicana axenic amastigotes and Plasmodium falciparum. Cytotoxicity was tested against HEK293 
Table 4.2. Cyclic Dimer 3-Alkyl Pyridinium Alkaloids.

\begin{tabular}{|c|c|c|c|}
\hline Structure & Name (Alkyl Chain Lengths) & Reported Activity* & Reference \\
\hline 22 & Cyclostellettamine $\mathrm{A}\left(\mathrm{C}_{12} / \mathrm{C}_{12}\right)$ & $\mathrm{B}, \mathrm{C}, \mathrm{N}, \mathrm{T}$ & 117 \\
\hline 23 & Cyclostellettamine $\mathrm{B}\left(\mathrm{C}_{12} / \mathrm{C}_{13}\right)$ & $\mathrm{B}, \mathrm{N}$ & 117 \\
\hline 24 & Cyclostellettamine $\mathrm{C}\left(\mathrm{C}_{13} / \mathrm{C}_{13}\right)$ & $\mathrm{B}, \mathrm{F}, \mathrm{N}$ & 117 \\
\hline 25 & Cyclostellettamine $\mathrm{D}\left(\mathrm{C}_{12} / \mathrm{C}_{14}\right)$ & $\mathrm{B}, \mathrm{F}, \mathrm{N}$ & 117 \\
\hline 26 & Cyclostellettamine $\mathrm{E}\left(\mathrm{C}_{13} / \mathrm{C}_{14}\right)$ & $\mathrm{B}, \mathrm{F}, \mathrm{N}$ & 117 \\
\hline 27 & Cyclostellettamine $\mathrm{F}\left(\mathrm{C}_{14} / \mathrm{C}_{14}\right)$ & $\mathrm{B}, \mathrm{F}, \mathrm{N}$ & 117 \\
\hline 28 & Cyclostellettamine $\mathrm{G}\left(\mathrm{C}_{12} / \mathrm{C}_{11}\right)$ & $\mathrm{B}, \mathrm{C}, \mathrm{T}$ & 118,119 \\
\hline 29 & Dehydrocyclostellettamine $\mathrm{D}\left(\mathrm{C}_{12} / \mathrm{C}_{14}\right)$ & $\mathrm{C}, \mathrm{T}$ & 118 \\
\hline 30 & Dehydrocyclostellettamine $\mathrm{E}\left(\mathrm{C}_{13} / \mathrm{C}_{14}\right)$ & $\mathrm{C}, \mathrm{T}$ & 118 \\
\hline 31 & Cyclostellettamine $\mathrm{H}\left(\mathrm{C}_{12} / \mathrm{C}_{10}\right)$ & B & 119 \\
\hline 32 & Cyclostellettamine $\mathrm{I}\left(\mathrm{C}_{13} / \mathrm{C}_{10}\right)$ & B & 119 \\
\hline 33 & Cyclostellettamine $\mathrm{K}\left(\mathrm{C}_{14} / \mathrm{C}_{10}\right)$ & B & 119 \\
\hline 34 & Cyclostellettamine $\mathrm{L}\left(\mathrm{C}_{14} / \mathrm{C}_{11}\right)$ & B & 119 \\
\hline 35 & $\left(\mathrm{C}_{10} / \mathrm{C}_{10}\right)$ & I & 121 \\
\hline 36 & Cyclohaliclonamine $\mathrm{A}\left(\mathrm{C}_{10} / \mathrm{C}_{10}\right)$ & $\mathrm{T}$ & 122 \\
\hline 37 & 8,8'-Diene-cyclostellettamine $\left(\mathrm{C}_{11} / \mathrm{C}_{11}\right)$ & $\mathrm{B}, \mathrm{F}$ & 123 \\
\hline 38 & $\left(\mathrm{C}_{11} / \mathrm{C}_{11}\right)$ & $\mathrm{C}, \mathrm{T}$ & 124 \\
\hline 39 & Njaoaminium $\mathrm{A}\left(\mathrm{C}_{10} / \mathrm{C}_{10}\right)$ & & 125 \\
\hline 40 & Njaoaminium $B\left(C_{10} / C_{10}\right)$ & $\mathrm{C}$ & 125 \\
\hline 41 & Njaoaminium $\mathrm{C}\left(\mathrm{C}_{10} / \mathrm{C}_{10}\right)$ & & 125 \\
\hline 42 & Cyclostellettamine $\mathrm{N}\left(\mathrm{C}_{9} / \mathrm{C}_{10}\right)$ & $\mathrm{B}, \mathrm{C}$ & 126 \\
\hline 43 & Cyclostellettamine $\mathrm{Q}\left(\mathrm{C}_{10} / \mathrm{C}_{11}\right)$ & $\mathrm{B}, \mathrm{C}$ & 126 \\
\hline 44 & $\left(\mathrm{C}_{8} / \mathrm{C}_{10}\right)$ & $\mathrm{B}, \mathrm{C}$ & 126 \\
\hline 45 & $\left(\mathrm{C}_{10} / \mathrm{C}_{10}\right)$ & $\mathrm{B}, \mathrm{C}$ & 126 \\
\hline 46 & $\left(\mathrm{C}_{10} / \mathrm{C}_{10}\right)$ & $\mathrm{B}, \mathrm{C}$ & 126 \\
\hline 47 & $\left(\mathrm{C}_{10} / \mathrm{C}_{10}\right)$ & $\mathrm{B}, \mathrm{C}$ & 126 \\
\hline 48 & $\left(\mathrm{C}_{10} / \mathrm{C}_{11}\right)$ & $\mathrm{B}, \mathrm{C}$ & 126 \\
\hline 49 & $\left(\mathrm{C}_{10} / \mathrm{C}_{11}\right)$ & $\mathrm{B}, \mathrm{C}$ & 126 \\
\hline 50 & $\left(\mathrm{C}_{10} / \mathrm{C}_{12}\right)$ & $\mathrm{B}, \mathrm{C}$ & 126 \\
\hline 51 & $\left(\mathrm{C}_{10} / \mathrm{C}_{12}\right)$ & $\mathrm{B}, \mathrm{C}$ & 126 \\
\hline
\end{tabular}

$* \mathrm{~B}=$ Antibacterial, $\mathrm{F}=$ Antifungal, $\mathrm{C}=$ Anticancer, $\mathrm{I}=$ Immunomodulatory, $\mathrm{N}=$ Neurotoxin, $\mathrm{T}=$ General Toxicity

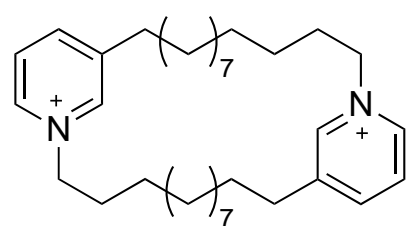

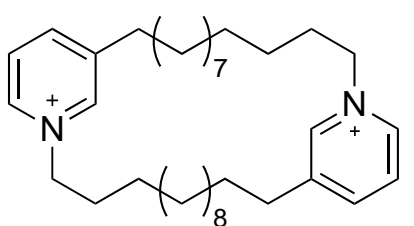

23

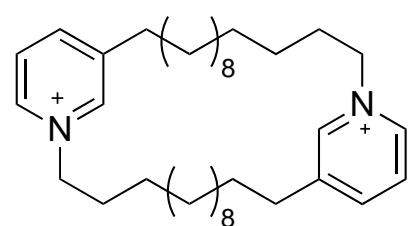


(N)

25

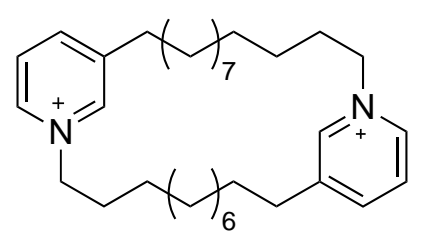

28

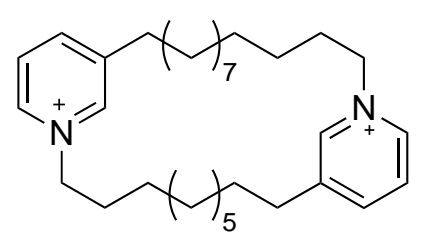

31

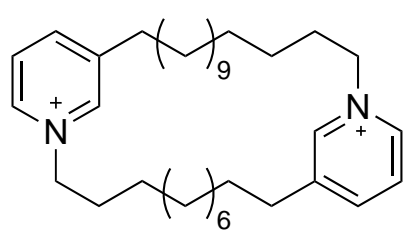

34

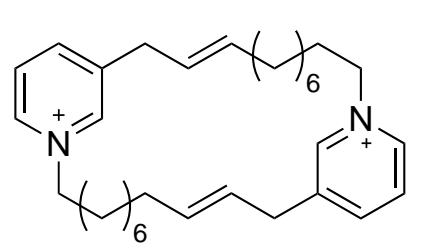

37

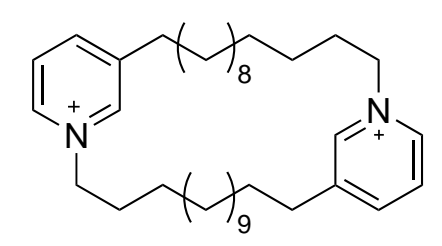

26

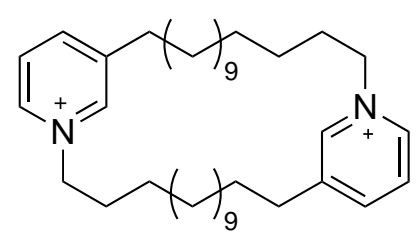

27

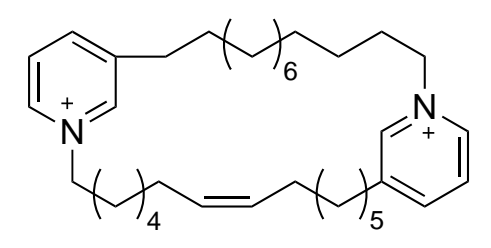

29

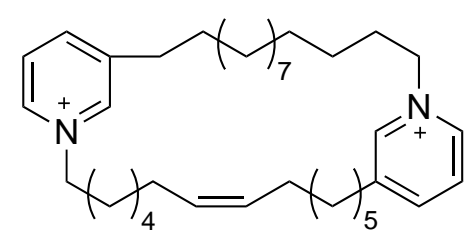

30

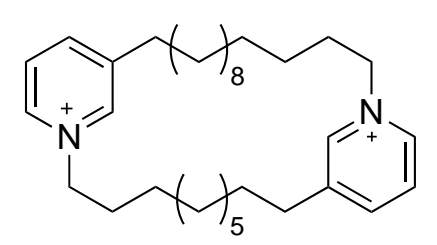

32

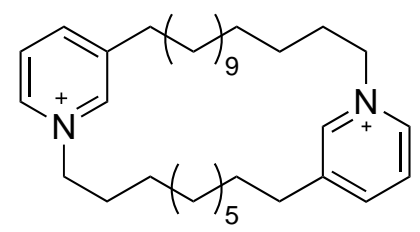

33

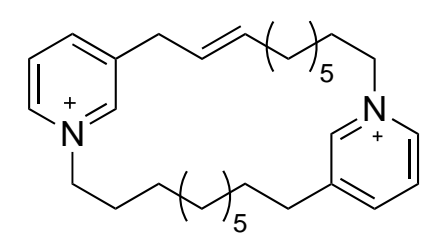

35

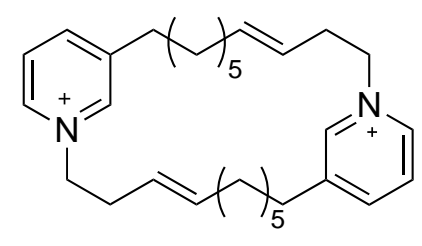

36

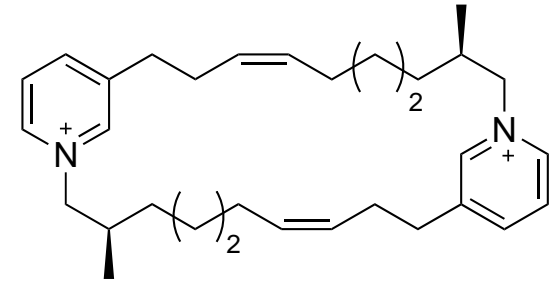

40

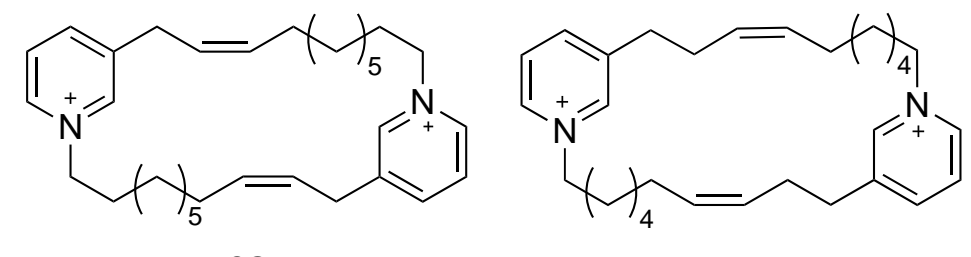

38

39

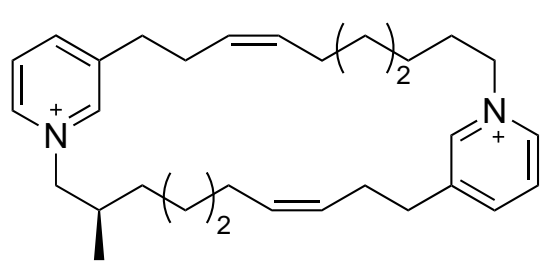

41

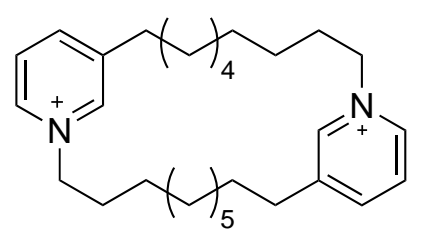

42

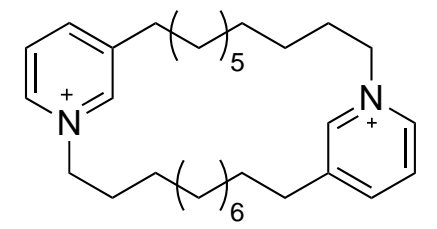

43

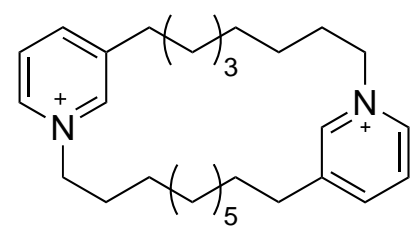

44 


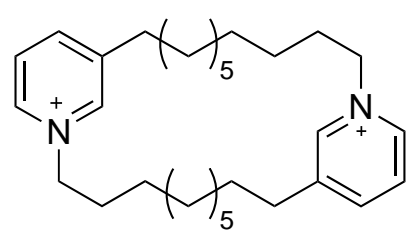

45

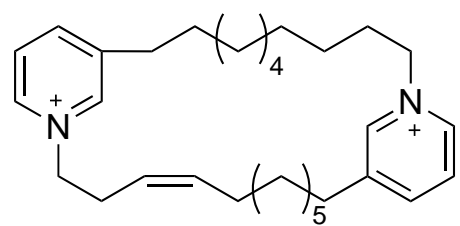

48

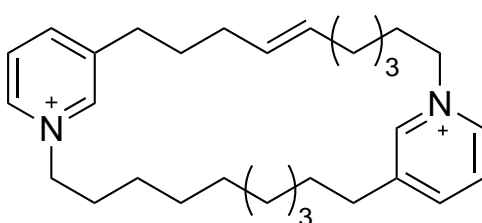

46

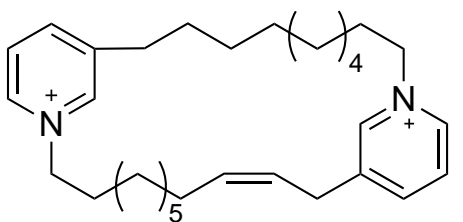

49

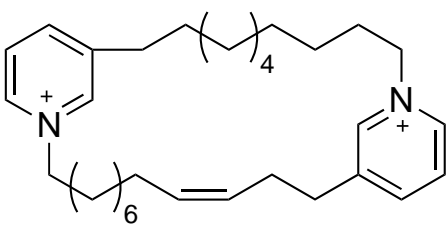

51

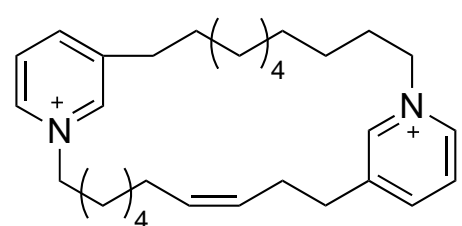

47

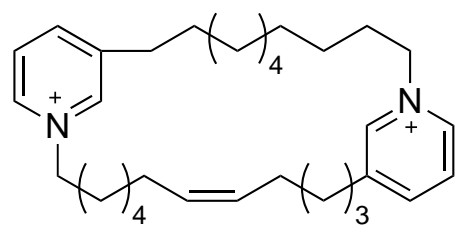

50

(human embryonic kidney) cell lines and this was used to calculate a selectivity index of the antiprotozoal activities. The assay showed that viscosamine had good activity against the Trypanosoma and Leishmania parasites with $\mathrm{EC}_{50}$ values ranging from 0.28 to $0.81 \mu \mathrm{M}$. Viscosamine proved to be the most active of the compounds tested against $P$. falciparum with an $\mathrm{EC}_{50}$ of $53 \mathrm{nM}$, however, this was still much less active than the reference compound chloroquine, which had an $\mathrm{EC}_{50}$ of $0.67 \mathrm{nM}$. The $\mathrm{IC}_{50}$ against HEK293 cells of $26 \mu \mathrm{M}$ showed that viscosamine is a potent antiprotozoal at concentrations that do not cause cytotoxicity, with a 493-fold selectivity for $P$. falciparum over HEK293 cells. ${ }^{130}$ Viscosamine was later renamed to viscosamine C, in order to follow the nomenclature of the cyclostellettamines and haliclocyclins. ${ }^{116}$

In 2006, when Teruya et al. isolated the dimeric cyclohaliclonamine A (36) from the sponge Haliclona sp., it was part of a mixture that included the trimeric cyclohaliclonamine B (53). Cyclohaliclonamine B is the trimeric 3-APA equivalent of cyclohaliclonamine A and so, like the dimer, the pyridinium moieties are connected by $\mathrm{C}_{10}$ alkyl chains with an E-geometry alkene at the C-9 to C-10 positions. ${ }^{122}$ In 2009 , Casapullo et al. isolated the dimeric $\mathbf{3 8}$ as well as its trimeric equivalent $\mathbf{5 4}$ from the marine sponge Haliclona sp. This trimer had $\mathrm{C}_{11}$ alkyl chains connecting the pyridinium units with $Z$-geometry alkenes in the $\mathrm{C}-15$ to $\mathrm{C}-16$ positions. Like its dimeric equivalent, trimer 54 showed weak cytotoxic activity with $\mathrm{IC}_{50}$ values greater than $20 \mu \mathrm{g} / \mathrm{mL}$ in assays against J774.A1 (murine macrophage), HEK293 (human embryonic kidney), and WEHI-164 (murine fibrosarcoma) cell lines. ${ }^{124}$ Currently, the 3-APA trimers that have been isolated are $C 3$ symmetric, meaning that they are comprised of three identical subunits. This is in contrast with the dimeric cyclostellettamines, which have been isolated as both $C 2$ symmetric and non-symmetric compounds. 
Table 4.3. Cyclic Trimer 3-Alkyl Pyridinium Alkaloids.

\begin{tabular}{cccc}
\hline Structure & Name (Alkyl Chain Lengths) & Reported Activity* & Reference \\
\hline $\mathbf{5 2}$ & Viscosamine C $\left(\mathrm{C}_{13}\right)$ & $\mathrm{P}, \mathrm{T}$ & 129 \\
$\mathbf{5 3}$ & Cyclohaliclonamine B $\left(\mathrm{C}_{10}\right)$ & $\mathrm{T}$ & 122 \\
$\mathbf{5 4}$ & $\left(\mathrm{C}_{11}\right)$ & $\mathrm{C}, \mathrm{T}$ & 124 \\
\hline
\end{tabular}

$* \mathrm{C}=$ Anticancer, $\mathrm{P}=$ antiprotozoal, $\mathrm{T}=$ General Toxicity

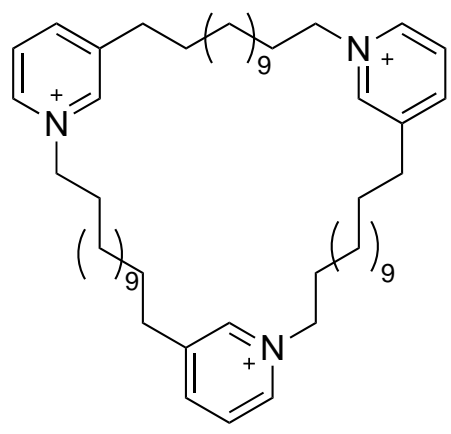

52

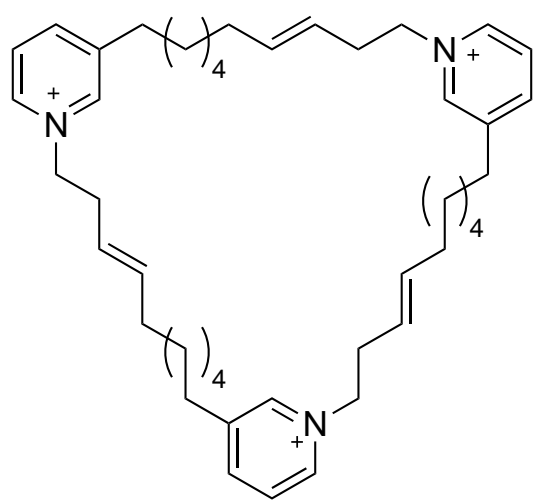

53

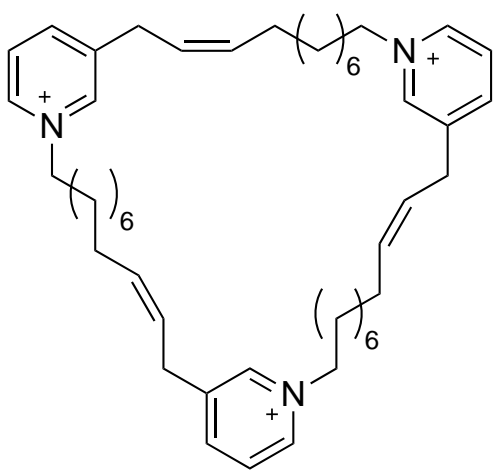

54

\subsubsection{Oligomers}

Three larger 3-APA oligomers have been isolated along with the mixture of dimer 36 and trimer $\mathbf{5 3}$ from Haliclona sp. These compounds were the tetrameric, pentameric and hexameric equivalents of $\mathbf{3 6}$ and $\mathbf{5 3}$. This meant the subunits of these oligomers were pyridinium units connected by $\mathrm{C}_{10}$ alkyl chains with an $E$-geometry alkene in the C-9 to C-10 positions. The mixture of 3-APAs proved to be difficult to separate and so they were tested for toxicity against brine shrimp as a mixture, this gave an $\mathrm{LD}_{50}$ of $65 \mu \mathrm{g} / \mathrm{mL}$. This is the only report of naturally isolated tetrameric, pentameric and hexameric 3-APAs to date. $^{122}$ 
Table 4.4. Cyclic Oligomer 3-Alkyl Pyridinium Alkaloids.

\begin{tabular}{cccc}
\hline Structure & Name (Subunits x Alkyl Chain Lengths) & Reported Activity* & Reference \\
\hline $\mathbf{5 5}$ & Cyclohaliclonamine $\mathrm{C}\left(4 \times \mathrm{x} \mathrm{C}_{10}\right)$ & $\mathrm{T}$ & 122 \\
$\mathbf{5 6}$ & Cyclohaliclonamine $\mathrm{D}\left(5 \times \mathrm{C}_{10}\right)$ & $\mathrm{T}$ & 122 \\
$\mathbf{5 7}$ & Cyclohaliclonamine $\mathrm{E}\left(6 \times \mathrm{C}_{10}\right)$ & $\mathrm{T}$ & 122 \\
\hline
\end{tabular}

$* \mathrm{~T}=$ General Toxicity

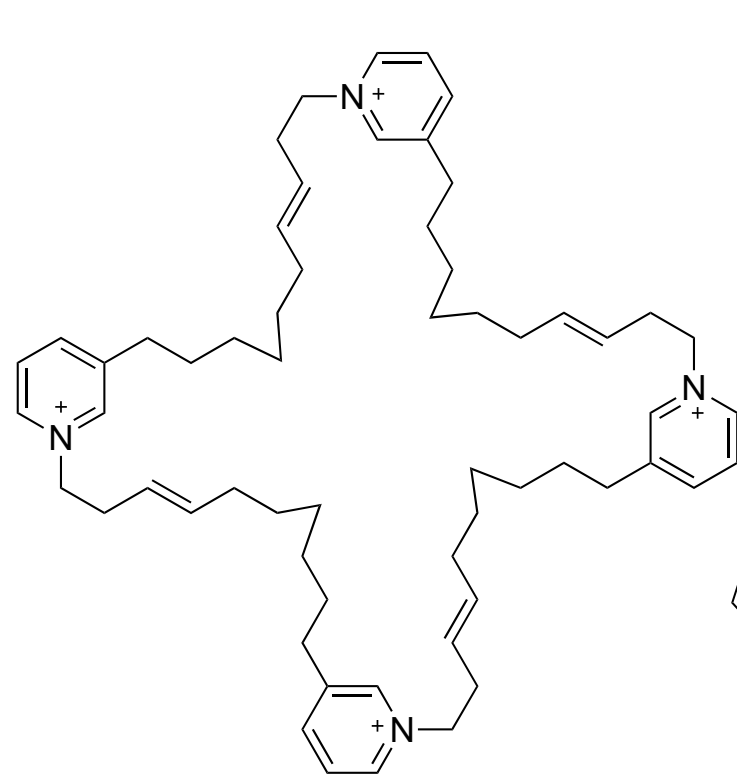

55

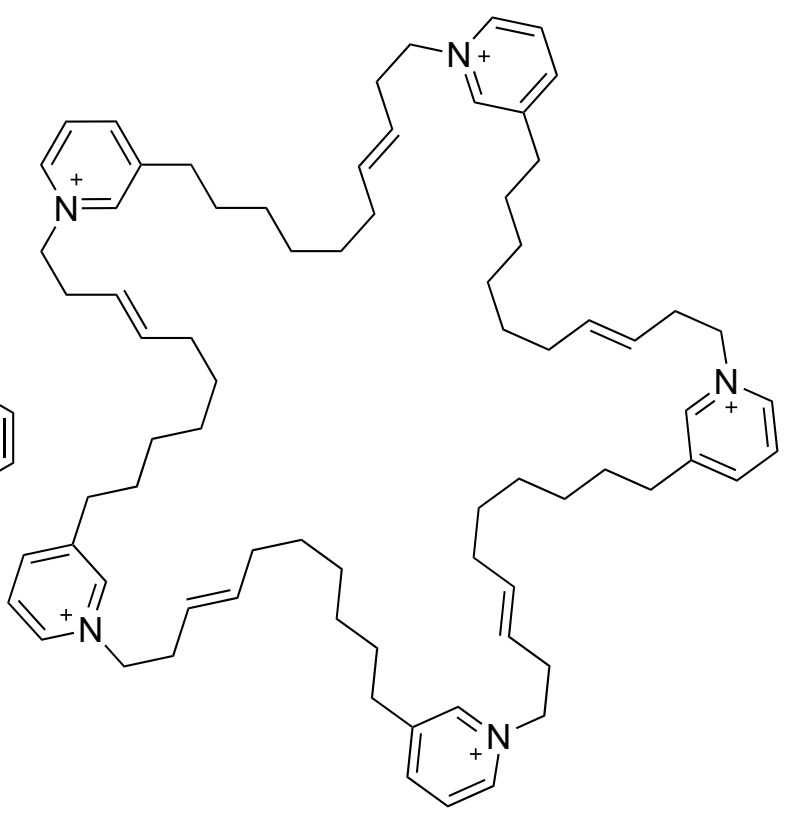

56

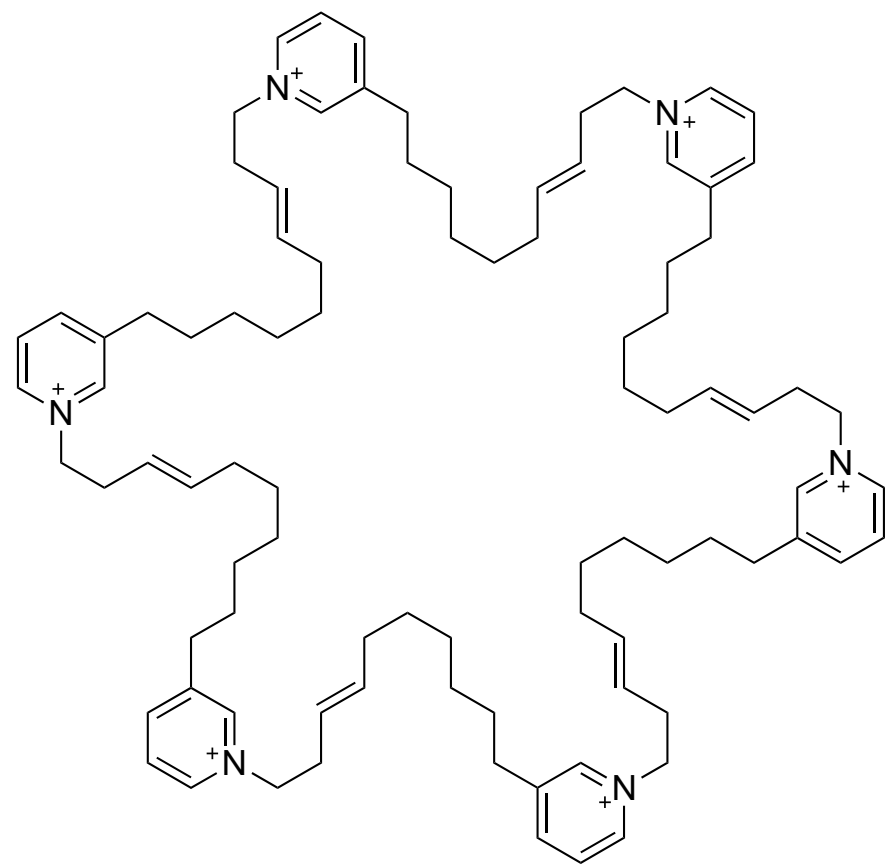

57 


\subsubsection{Structural Elucidation}

Despite their simplicity, structural elucidation of cyclic 3-APAs can be a difficult task. Most cyclic 3-APAs produce very similar NMR spectra, especially in the ${ }^{1} \mathrm{H}$ NMR spectrum. This usually consists of aromatic resonances from the 1,3-substituted pyridinium/s, which includes a singlet that is the most downfield (H-2), two doublets (H-4 and H-6) and a doublet of doublets or triplet that is the most upfield of the aromatic resonances (H-5), these all occur between 7.9 and $9.3 \mathrm{ppm}$ (see Figure 4.2 for compound numbering). Next in the ${ }^{1} \mathrm{H}$ NMR spectrum are the methylene units adjacent to the pyridinium nitrogen ( $\mathrm{H}-7$, a triplet between 4.5 and $4.9 \mathrm{ppm})$ and the 3-position carbon $(\mathrm{H}-\mathrm{n}+1$, a triplet between 2.8 and $3.0 \mathrm{ppm})$, as well as the methylenes adjacent to them (H-8 between 1.9 and 2.1 and H-n between 1.6 and 1.8). Finally, there is a methylene envelope of all the remaining methylene units that are in a similar environment (a broad unresolved resonance between 1.0 and $1.4 \mathrm{ppm}$ ). This similarity across the ${ }^{1} \mathrm{H}$ NMR spectra is broken up when an alkene is present producing alkene resonances (around 5.3 to $5.7 \mathrm{ppm}$ ) and shifting the allylic methylenes downfield, however a methylene envelope will still usually be present (this can make it hard to determine the exact position of the alkene). The spectra can also be more distinctive in the case of a non-symmetric 3-APA, the variation in the alkyl chains can cause differences in the ${ }^{1} \mathrm{H}$ NMR spectrum, allowing the various subunits to be distinguished.

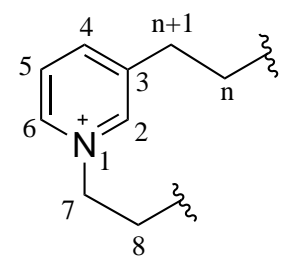

Figure 4.2. Compound numbering of an exemplary cyclic 3-APA, $\mathrm{n}=$ the number of carbon atoms in the structure.

The ${ }^{13} \mathrm{C}$ NMR spectrum can confirm the environments inferred from the proton spectrum as well as revealing the presence of the fully substituted carbon of the pyridinium (C-3). The ${ }^{13} \mathrm{C}$ NMR spectrum can also be very important in the determination of the geometry of any double bond that is present, as the allylic methylene chemical shifts in the carbon spectrum can be used to distinguish between an $E$-geometry (from 30 to $35 \mathrm{ppm}$, usually around $33 \mathrm{ppm}$ ) and Z-geometry (from 26-30 ppm, usually around 27 ppm). ${ }^{122,126,131-134}$ The COSY spectrum of a 3-APA (especially one that is symmetric) will provide little information on the structure, methylene units adjacent to functional groups can be identified but they will eventually couple to the methylene envelope preventing further determination of the position of that functional group. ${ }^{117}$ TOCSY experiments can be useful in distinguishing methylene units in the alkyl chain and can also be used to prove that substructures identified (the 1,3-substituted pyridinium structure and any alkene groups) are part of the same compound structure. ${ }^{108}$ The multiplicityedited HSQC will clearly show whether any methyl groups are present in a 3-APA while 
providing information on the direct attachments of protons to carbons. The HMBC experiment is one of the most important NMR experiments for structural elucidation of 3-APAs, the long-range information can indicate which signals in the ${ }^{1} \mathrm{H}$ NMR spectrum are from $\mathrm{H}-2, \mathrm{H}-4, \mathrm{H}-5$ and $\mathrm{H}-6$ in the pyridinium ring. Also, the HMBC information can help with connecting two functional group substructures if correlations between the key resonances of these groups can be seen in the HMBC. ${ }^{117,124}$

The most essential tool for determining the final structure of 3-APAs is mass spectrometry (MS). ${ }^{105,127,135}$ This is able to provide information of the molecular mass of the 3-APA that, in turn, can be used to deduce the length of the alkyl chain. Further information that can be gained from MS is the number of subunits in the 3-APA structure (e.g. monomer, dimer, trimer etc.). ${ }^{122}$ The mass spectrum of some compounds is complicated by the M+ mass to charge ratio $(\mathrm{m} / \mathrm{z})$ reported for equivalent monomers, dimers, trimers and oligomers being the same value due to each subunit being charged. One can, however, distinguish between these by looking at the higher isotopomer peaks, these will also be divided by the total charge of the molecule thus altering the spacing between these peaks. For example, a monomer will have a normal $\mathrm{M}+$ followed by an $\mathrm{M}+1$ and an $\mathrm{M}+2$, a dimer will have an $\mathrm{M}+$ followed by an $\mathrm{M}+\frac{1}{2}$ and an $\mathrm{M}+1$, a trimer will have an $\mathrm{M}+$ followed by an $\mathrm{M}+\frac{1}{3}$ and an $\mathrm{M}+\frac{2}{3}$ etc. MS can also be used to determine the purity of a 3-APA, which might not be clear from NMR if the impurity is another 3-APA. ${ }^{114}$

The mass spectrum can be further complicated by the presence of counter ions in the 3-APA and also by fragmentations occurring in the mass spectrometer. ${ }^{113,117}$ A common in-source generated fragment that is found in mass spectra of cyclic 3-APAs is the product of a Hofmann elimination, which produces an $[\mathrm{M}-\mathrm{H}]^{(n-1)+}$ as the major product, where $n$ is the number of charged subunits in the 3-APA (Figure 4.3). ${ }^{113,117,119,122,124,127,136}$ In fact, only the Hofmann elimination product is observed in the mass spectrum of some cyclic 3-APAs. ${ }^{115}$ The occurrence of this Hofmann elimination product is thought to be dependant on the counter-ion of the 3-APA. ${ }^{136}$ 3-APAs give characteristic and reliable tandem mass spectrometry (MS/MS) fragmentations. MS/MS has been used extensively to determine the length of the alkyl chains of 3-APAs and can also be used in order to identify or confirm the substructures of a 3-APA. ${ }^{11,112,117,119,124,126,137}$ Two well known fragments that occur from 3-APAs during MS/MS are the onium reaction and McLafferty rearrangement products (Figure 4.3). When only one of these fragmentations occurs in a cyclic 3-APA the mass observed will not necessarily change, however, when a combination of fragmentations occur, masses characteristic of the alkyl chain length can be observed. ${ }^{111,127}$ MS in combination with MS/MS is also useful in the determination of the position of a double bond after an oxidative cleavage has been performed. ${ }^{118,135}$ 


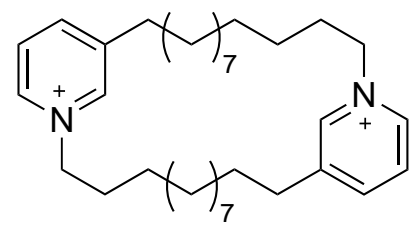<smiles>C=CCC(C)(C)CCc1ccc[n+](CCCCC(C)(C)C)c1</smiles>

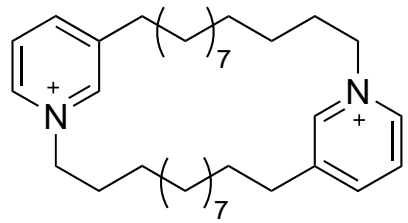

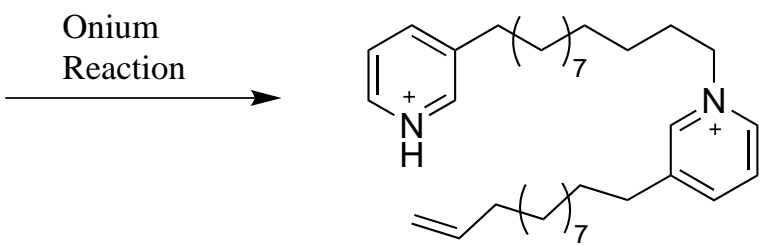<smiles>CCCCCCCCCCCCCCCCC[n+]1cccc(CCCCC)c1</smiles>

\section{McLafferty Rearrangement}

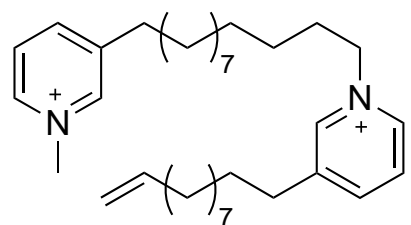

Figure 4.3. Examples of Hofmann elimination, onium reaction and McLafferty rearrangement fragmentations modelled on cyclostellettamine A (22).

\subsection{3-APAs Isolated}

In this study, one known and two new 3-APA cyclic monomers were isolated from the marine sponge Haliclona sp. This is also the first report of unsaturated 3-APA cyclic monomers to our knowledge. The 3-APAs were found in the more polar $20 \%, 40 \%$ and $60 \%$ acetone in water fractions from crude separation on an HP20 column. This was indicated in the ${ }^{1} \mathrm{H}$ NMR spectra (Figure 3.2) that all showed the same resonances characteristic of 3-APAs (see Section 4.3.5 for characteristic 3-APA resonances), and the fractions all had similar approximate $\mathrm{IC}_{50}$ values of $1.5 \mu \mathrm{g} / \mathrm{mL}$ when tested against S. cerevisiae.

Further purification of the $20 \%$ acetone in water fraction (VD1_96A) was attempted using HP20ss, however the 3-APAs all eluted in the 50\% methanol in water wash of the column (VD2_373B). Diol was then used to further purify the 3-APAs and they were collected in eight separate fractions over the $25 \%, 50 \%$ and $75 \%$ methanol in ethyl acetate elutions (VD2_384G-N). Of these fractions, the sample with the largest mass was VD2_384G with $6.7 \mathrm{mg}$, so this sample was chosen to be purified further using reversedphase (C18) HPLC. A gradient of acetonitrile in water (both with $0.1 \%$ formic acid) was used to separate out the 3-APAs. The 3-APAs were collected as samples VD2_394F and VD2_394G with masses of 1.1 and $2.3 \mathrm{mg}$, respectively. Full sets of NMR spectra were run on these compounds. The isolation procedure of VD2_394 F and G from MNP_0999 is shown in Scheme 4.1. 


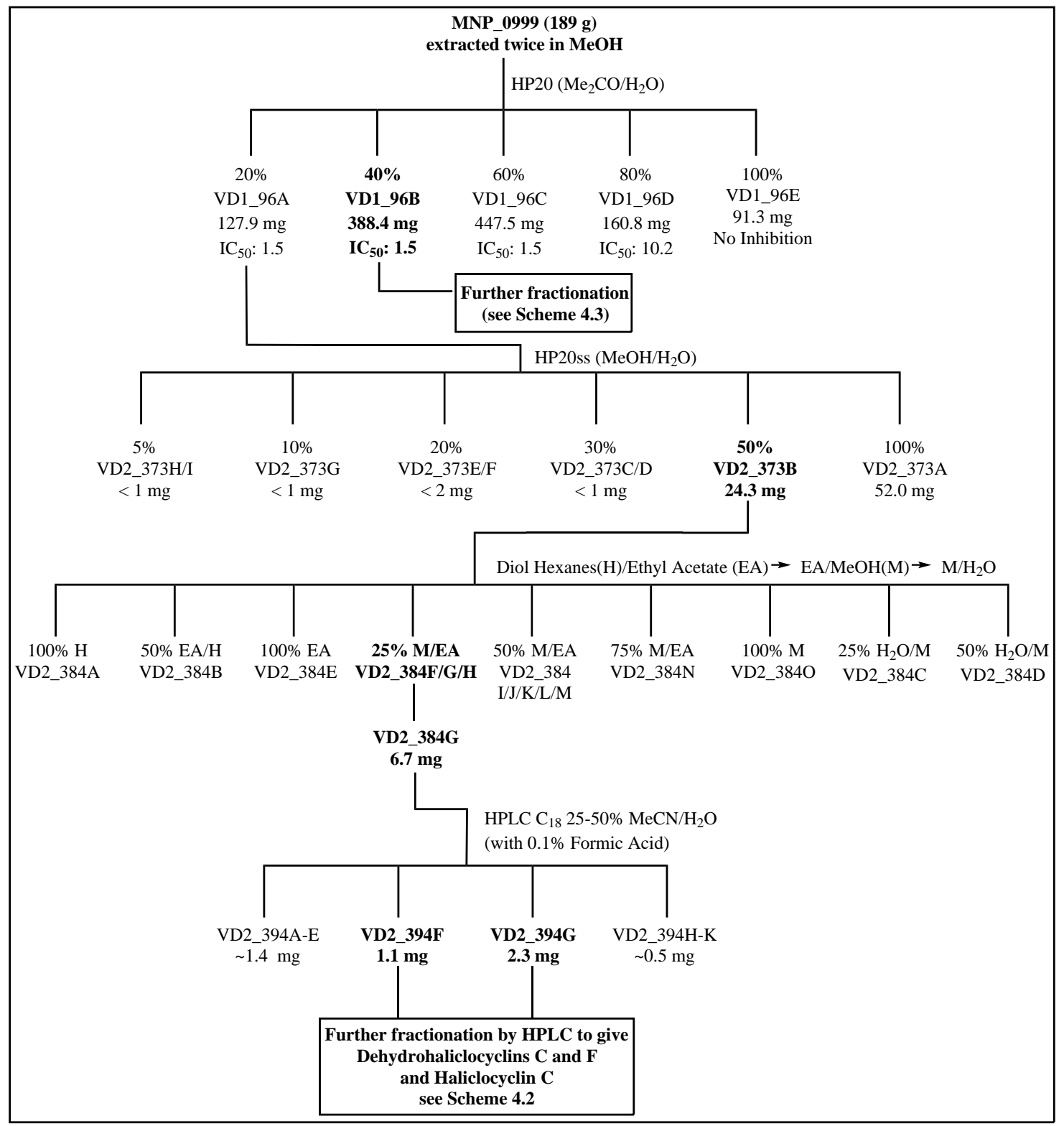

Scheme 4.1. Isolation procedure for 3-APAs from the sponge MNP_0999. Note that $\mathrm{IC}_{50}$ values are approximate $(\mu \mathrm{g} / \mathrm{mL})$.

The structure of VD2_394F was elucidated as will be described in section 4.4.1, this was a $\mathrm{C}_{18}$ unsaturated monomer and was named dehydrohaliclocyclin C (58). VD2_394F was further purified using the same conditions on reversed-phase HPLC (VD3_86B) for biological testing to be performed and to unambiguously establish the geometry of the double bond. Over the course of NMR experiments used to determine the geometry of the double bond VD3_86B accumulated some impurity and so needed to be cleaned up again using reversed-phase HPLC. This purification used 53\% methanol in water (with $0.1 \%$ formic acid in both), this produced the final compound VD4_33C that was identified as the new compound dehydrohaliclocyclin C (58). Quantitative NMR was performed on this sample to give a final mass of $0.36 \mathrm{mg}$ and this was submitted for biological testing against the clinically relevant fungus $C$. albicans.

VD2_394G was found to be a mixture of 3-APAs and so was further purified using 


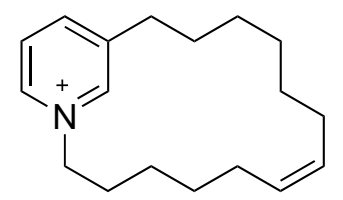

58

reversed-phase HPLC with the same acetonitrile in water gradient used previously. The 3-APAs came off as one peak that showed two shoulders in the UV/Vis trace, indicating that there may be at least three different 3-APAs in the sample. The major peak was collected as two fractions in order to try to separate out the 3-APAs better. The first part of this peak (VD3_87C) showed a cleaner ${ }^{1} \mathrm{H}$ NMR spectrum than the second half (VD3_87D), VD3_87C appeared to also have a larger mass than VD3_87D as shown by the ${ }^{1} \mathrm{H}$ NMR spectra. A full set of NMR experiments were run on VD3_87C and these showed that there were two 3-APAs that were the major compounds in this sample. The mass spectrum of this fraction suggested that the compounds were a $\mathrm{C}_{19}$ unsaturated monomer that was named dehydrohaliclocyclin $\mathrm{F}(\mathbf{5 9})$, and a $\mathrm{C}_{18}$ saturated monomer that was the known compound haliclocyclin $\mathrm{C}(\mathbf{2 0})$.

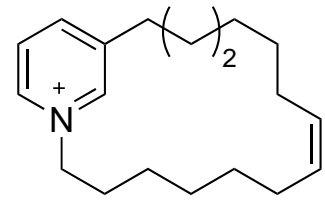

59

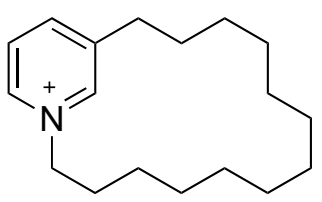

20

In order to separate out the two compounds in fraction VD3_87C (for biological testing and to determine the position and geometry of the alkene), reversed-phase HPLC was utilised using an isocratic elution of 53\% methanol in water (both containing $0.1 \%$ formic acid). The 3-APA peaks in the UV/Vis trace were overlapping but the majority of the mass was able to be separated. A full set of NMR experiments were run on the samples VD4_32A (the $\mathrm{C}_{19}$ unsaturated monomer dehydrohaliclocyclin F, 59) and VD4_32B (the $\mathrm{C}_{18}$ saturated monomer haliclocyclin $\mathrm{C}, 20$ ) that allowed their structures to be elucidated. The isolation procedure for the dehydrohaliclocyclins $\mathrm{C}$ and $\mathrm{F}$ and haliclocyclin $\mathrm{C}$ is shown in Scheme 4.2. Following the structural elucidation of these 3-APAs, a successful oxidative cleavage of the alkene of $\mathbf{5 9}$ was performed in order to determine the location of the double bond using MS. Quantitative NMR was performed on these samples to give a final mass of $0.25 \mathrm{mg}$ for $\mathbf{5 9}$ (dehydrohaliclocyclin F) and $0.16 \mathrm{mg}$ for 20 (haliclocyclin C). These samples were then submitted for biological testing against the clinically relevant fungus $C$. albicans.

Attempts were also made to purify the $40 \%$ acetone in water fraction (VD1_96B) from the crude HP20 column separation. An HP20ss column was the first stage of purification and 3-APAs eluted off the column throughout the $60 \%, 70 \%, 80 \%$ and $100 \%$ methanol in water elutions. The cleanest fraction that also had the largest mass was the $80 \%$ methanol in water fraction that had been collected in bulk (VD2_356C), $22.4 \mathrm{mg}$ of this was 


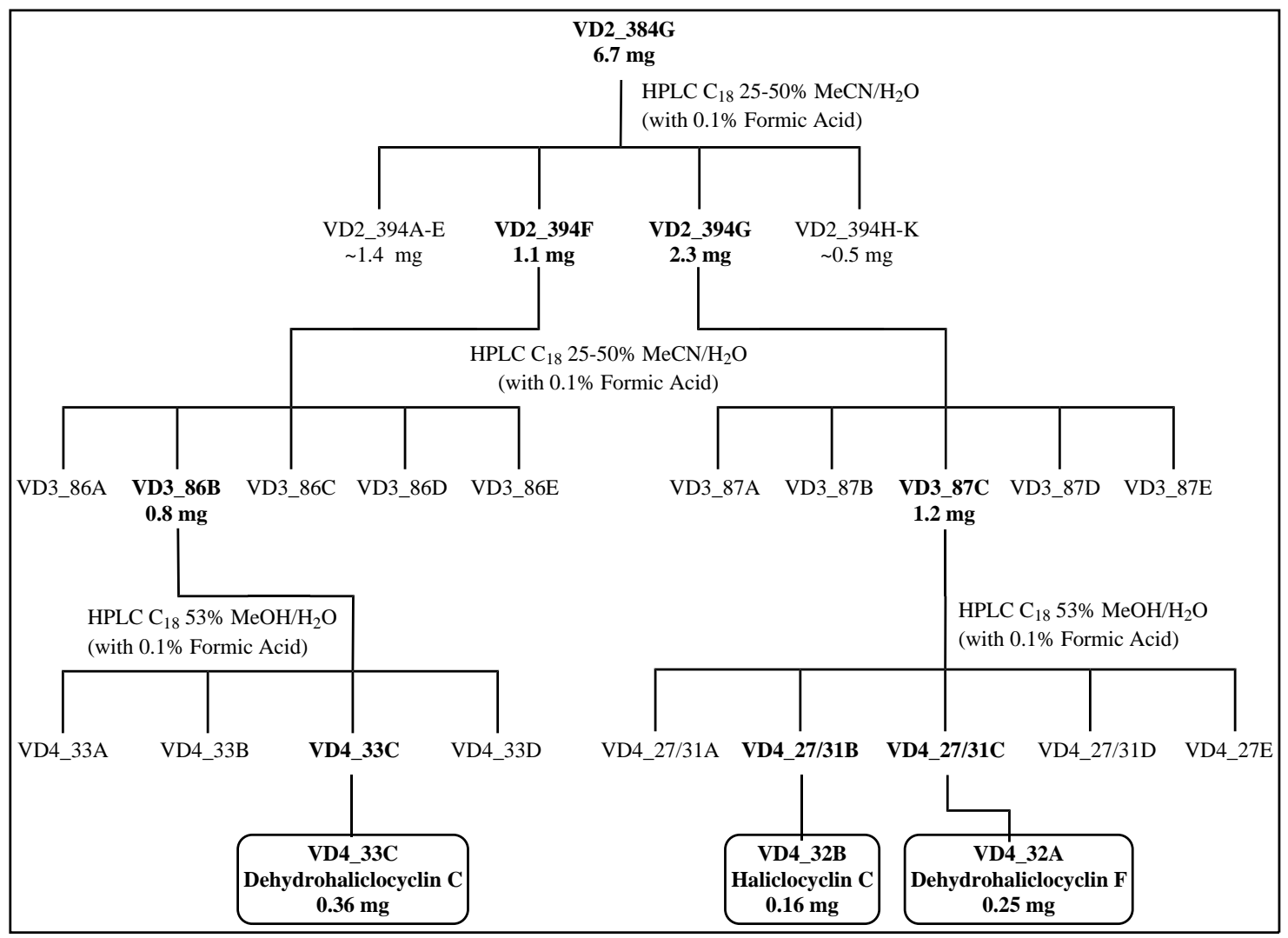

Scheme 4.2. Isolation procedure for the dehydrohaliclocyclins $C(\mathbf{5 8})$ and $F(\mathbf{5 9})$ and haliclocyclin C (20) from the fraction VD2_384G that was isolated from the sponge MNP_0999 (see Scheme 4.1).

recovered. When tested against $S$. cerevisiae this sample had an $\mathrm{IC}_{50}$ of $2.4 \pm 1.0 \mu \mathrm{g} / \mathrm{mL}$. This sample was then subjected to semi-preparative reversed-phase HPLC using an isocratic $62 \%$ methanol in water elution (with $0.1 \%$ formic acid in both solvents) with an 80\% methanol strip incorporated in the run. The UV/Vis and ELSD traces showed three broad overlapping peaks with low intensities that became hard to distinguish as injection volumes were increased. ${ }^{1} \mathrm{H}$ NMR was run on all of the fractions collected and 3-APAs were identified in nine out of the ten fractions. Fractions VD4_43E and F with masses of 3.3 and $3.8 \mathrm{mg}$, respectively, were recombined and renamed VD4_46A on the basis of ${ }^{1} \mathrm{H}$ NMR. Poor solubility occurred in these 3 -APA samples when running ${ }^{1} \mathrm{H}$ NMR so the counter-ion was exchanged using $\mathrm{NaCl}$, this gave a much clearer spectrum of the sample (VD4_47A). A full set of NMR experiments were run on this sample, these showed that the sample was not completely pure as some of the ${ }^{13} \mathrm{C}$ NMR resonances were broad and there were minor resonances in the ${ }^{1} \mathrm{H}$ and ${ }^{13} \mathrm{C}$ NMR spectra. The contaminants appear to be other 3-APAs thus demonstrating the difficulty in separating these compounds. A potential structure was proposed for the major component of VD4_47A, however, the mass spectrum was not able to confirm this. No clear M+ peak was detected in the mass spectrum making it impossible to identify the molecular formula of the major compound in VD4_47A. This meant that the final structure of this 3-APA was not able to be solved due to time constraints. The isolation of VD4_47A from the sponge MNP_0999 is shown in Scheme 4.3. 


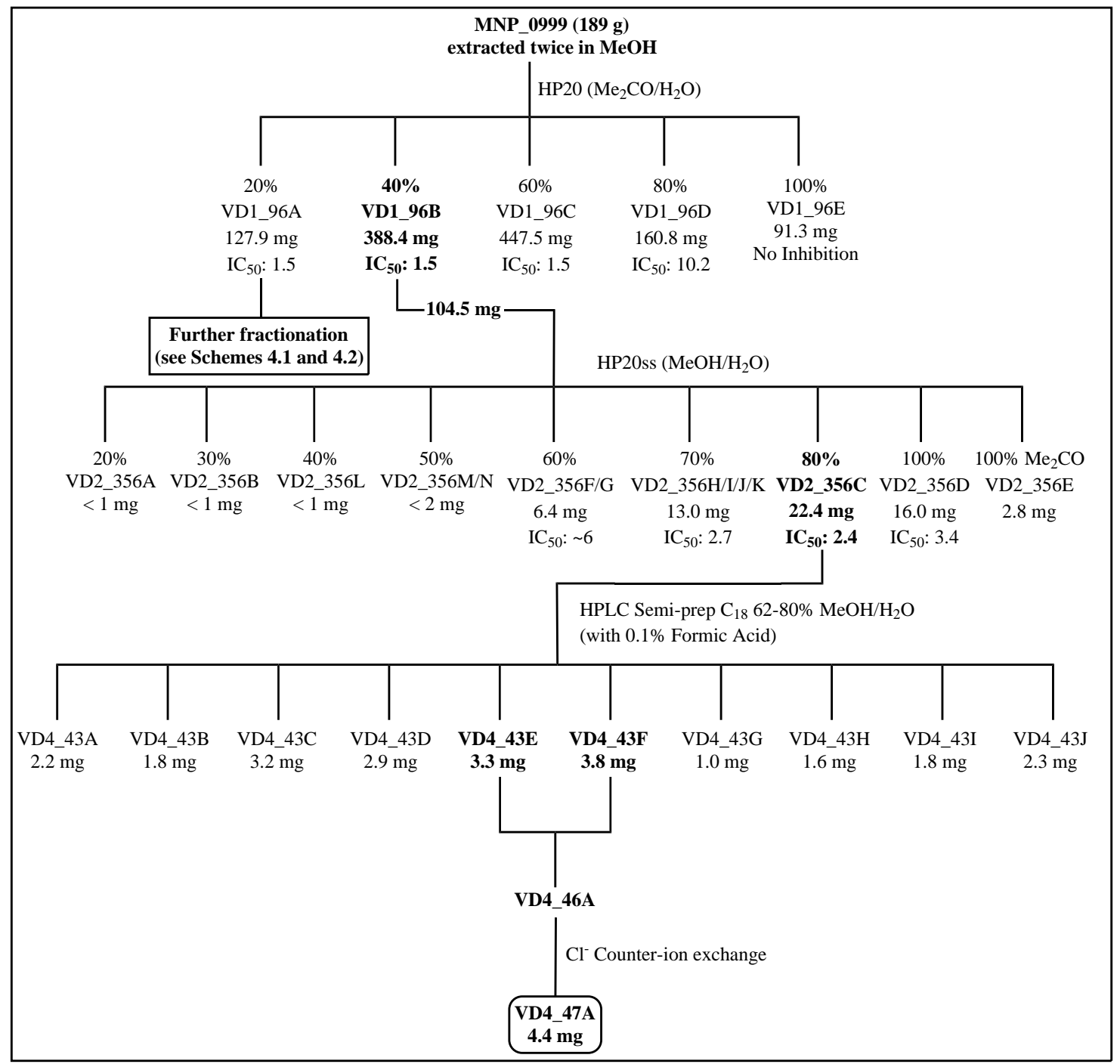

Scheme 4.3. Isolation procedure for unknown 3-APA (VD4_47A) from the sponge MNP_0999. Note that $\mathrm{IC}_{50}$ values are approximate $(\mu \mathrm{g} / \mathrm{mL})$.

\subsubsection{Dehydrohaliclocyclin C}

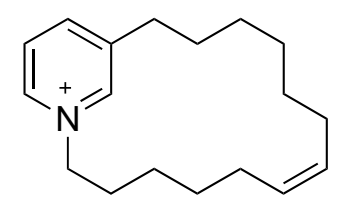

58

Dehydrohaliclocyclin C (58) was isolated as a colourless oil. Positive ion mode HRESIMS showed a singly charged molecular ion of $\mathrm{m} / \mathrm{z} 258.2216(\mathrm{~m} / \mathrm{z}, \Delta=0.0000)$ that indicated a molecular formula of $\mathrm{C}_{18} \mathrm{H}_{28} \mathrm{~N}^{+}$with an IHD of 5.5; this indicated a natively charged structure with six double bonds and/or cyclic moieties. The ${ }^{13} \mathrm{C}$ NMR spectrum was used in combination with the ${ }^{1} \mathrm{H}$ NMR and multiplicity-edited HSQC spectra to reveal four aromatic methines $\left(\delta_{\mathrm{C}} 145.2,144.1,143.6\right.$, and 128.5), one aromatic non-protonated carbon $\left(\delta_{\mathrm{C}} 143.4\right)$, two alkene methines $\left(\delta_{\mathrm{C}} 130.5\right.$ and 129.8), one deshielded methylene $\left(\delta_{\mathrm{C}} \quad 62.7\right)$ and 10 other methylene groups $\left(\delta_{\mathrm{C}} 32.3,31.1,29.0\right.$, $28.41,28.36,27.3,27.1,26.6,25.2$ and 25.1). The resonances of protons attached to 
these carbons, determined using the multiplicity-edited HSQC spectrum, can be seen in Table 4.5. Proton integration indicated that all of the hydrogen atoms in the formula were attached to carbon, therefore there were no exchangeable protons in the structure. The absence of any methyl groups in the ${ }^{13} \mathrm{C}$ NMR and multiplicity-edited HSQC spectra suggested a cyclic structure, this was in agreement with the molecular formula proposed (and the degrees of unsaturation calculated from this) and was confirmed by the NMR data described below.

The multiplicity of the aromatic protons in the ${ }^{1} \mathrm{H}$ NMR spectrum $\left(\delta_{\mathrm{H}} 9.60\right.$, broad doublet; 8.83, singlet; 8.17, doublet; 8.03, broad triplet) indicated a 1,3-disubstituted benzene-like structure. The ${ }^{13} \mathrm{C}$ NMR spectrum only contained five aromatic carbon resonances, this indicated the presence of an atom other than carbon at the 1-position. In the HMBC spectrum, it could be seen that the proton at $8.83 \mathrm{ppm}(\mathrm{H}-2)$ correlated to the downfield methylene at $62.7 \mathrm{ppm}(\mathrm{C}-7)$, the most downfield regular methylene at $32.3 \mathrm{ppm}$ (C-19) and the non-protonated aromatic carbon at $143.4 \mathrm{ppm}(\mathrm{C}-3)$. This indicated that this proton was attached to the carbon at the 2-position of the aromatic ring as it correlated to both of the methylene substituents and the 3-position. The most downfield proton at $9.60 \mathrm{ppm}$ (H-6) also showed an HMBC correlation to C-7, suggesting that this proton was attached to the carbon on the other side of the 1-position. The proton resonance at $8.17 \mathrm{ppm}(\mathrm{H}-4)$ showed an HMBC correlation to the methylene substituent $\mathrm{C}-19$, indicating that this proton was attached to the carbon at the 4-position of the aromatic ring. This left the aromatic methine at $8.03 \mathrm{ppm}$, with the most upfield shift in both the ${ }^{1} \mathrm{H}$ and ${ }^{13} \mathrm{C}$ NMR spectra, to be in the 5-position of the aromatic ring. This proposed structure was consistent with the observed proton multiplicities and COSY correlations, which showed long-range coupling for most of the aromatic protons (Figure 4.4).

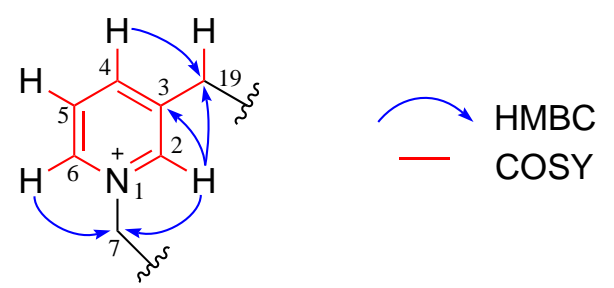

Figure 4.4. Key COSY and HMBC correlations establishing the 3-APA pyridinium substructure of dehydrohaliclocyclin C.

From the downfield shifts of the resonances of the protons in the vicinity of the 1-position (H-2, H-6 and H-7), a charged nitrogen was proposed to be in the 1-position, this would make a 1,3-substituted pyridinium structure. A search of the MarinLit database of a 1,3-substituted pyridinium structure showed that this was a well known class of compounds with very similar aromatic resonances in the ${ }^{1} \mathrm{H}$ NMR spectrum: the 3-alkyl pyridinium alkaloids. This 3-APA was unusual as the $\mathrm{H}-2$ singlet is usually the most downfield resonance in the ${ }^{1} \mathrm{H}$ NMR spectrum (8.85-9.14 ppm) followed by the H-6 doublet (8.75-8.98 ppm), however, in $\mathbf{5 8}$ this was not the case. The H-6 doublet resonance of $\mathbf{5 8}$ had been shifted downfield to $9.60 \mathrm{ppm}$ and was followed by the typical H-2 singlet 
resonance at $8.83 \mathrm{ppm}$. The reason for this difference between the spectra has not been determined. All other pyridinium resonances of this compound were consistent with a 3-APA structure (see Section 4.3.5).

A contiguous chain of methylenes ( $\mathrm{H}-7$ to $\mathrm{H}-11)$ were then established from mutual, resolved COSY correlations. Allylic proton H-11 showed further COSY correlations to the alkene proton at $5.17 \mathrm{ppm}$ that coupled to the other alkene proton at $5.23 \mathrm{ppm}$, placing the alkene at $\mathrm{C}-12$ and $\mathrm{C}-13$. The alkene proton $\mathrm{H}-13$ coupled to the methylene proton at $1.94 \mathrm{ppm}(\mathrm{H}-14)$ that led to another contiguous chain of methylenes ( $\mathrm{H}-14$ to $\mathrm{H}-19)$ established from mutual, resolved COSY correlations. This allowed the final structure of $\mathbf{5 8}$ to be proposed (Figure 4.5). The structure proposed based on this COSY information is further supported by the remaining HMBC correlations. This structure, which was able to be proposed entirely on the basis of NMR data, is also in agreement with the molecular formula proposed on the basis of mass spectral data.

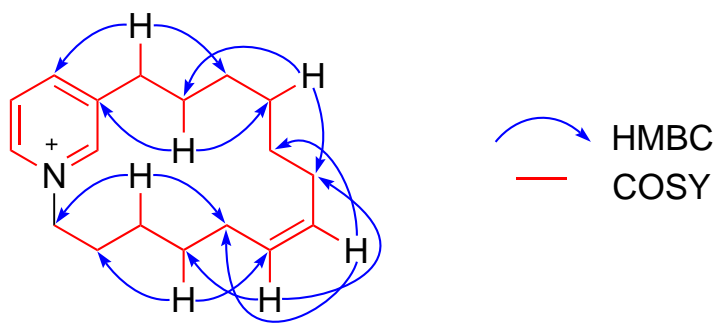

Figure 4.5. Key COSY and HMBC correlations establishing the structure of dehydrohaliclocyclin C (58).

It is very unusual for the alkyl chain resonances in a 3-APA to be completely resolved using the COSY spectrum, even at $600 \mathrm{MHz}$, the methylene group resonances usually overlap in the ${ }^{1} \mathrm{H}$ NMR spectrum to give a methylene envelope. In this case, the position of the double bond in the alkyl chain seems to have shifted the proton resonances enough so that each one could be distinguished from the other in the ${ }^{1} \mathrm{H}$ NMR spectrum (Figure 4.6). This was clearly shown by the individual, closely spaced multiplets in the ${ }^{1} \mathrm{H}$ NMR spectrum that all integrated for two protons, with the exception of the multiplet from 1.98 to $1.92 \mathrm{ppm}$ that integrated for four protons (as the proton resonances at 1.94 and $1.96 \mathrm{ppm}$ were overlapping but distinguishable in the 2D NMR spectra). The proton resonances from the alkene were also clearly distinguished in the ${ }^{1} \mathrm{H}$ NMR spectrum as two separate multiplets that integrated for one proton each.

Several approaches were initially taken to determine the geometry of the double bond. The first was to look at the ${ }^{13} \mathrm{C}$ chemical shifts of $\mathrm{C}-11$ and $\mathrm{C}-14\left(\delta_{\mathrm{C}} 27.1\right.$ and 25.2, respectively) that suggested a $Z$-geometry. ${ }^{122,126,131-134}$ The next approach used a 1D NOESY to see if the two alkene protons had diagnostic through-space (nOe) correlations. It was expected that if an alkene had a $Z$-geometry the two alkene protons would share nOe correlations and if the alkene had an $E$-geometry, there would be no nOe correlations between the protons. Furthermore, if the alkene had an $E$-geometry, nOe 


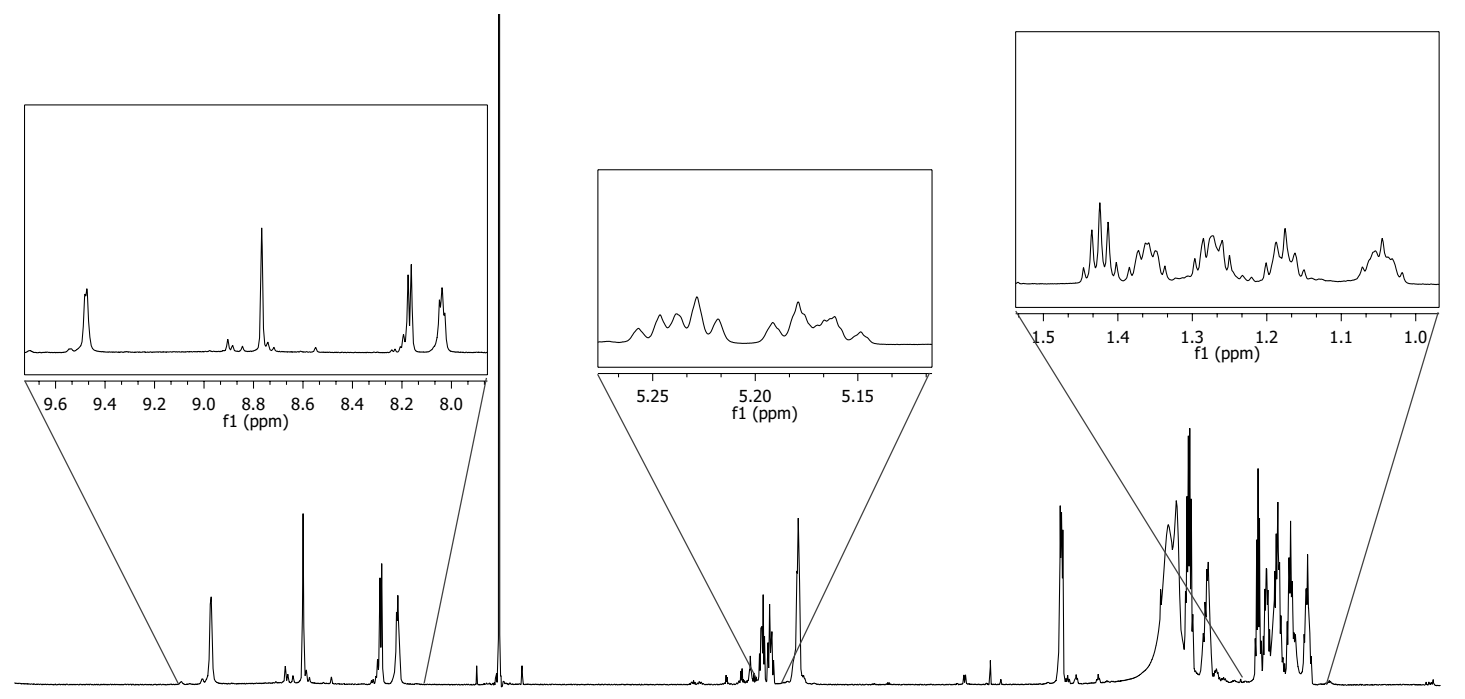

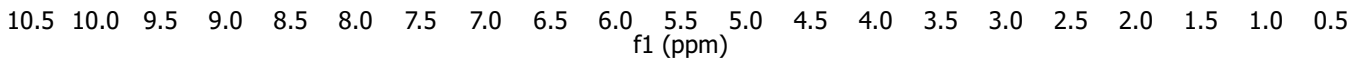

Figure 4.6. ${ }^{1} \mathrm{H}$ NMR spectrum of dehydrohaliclocyclin $\mathrm{C}$ showing expanded views of important regions $\left(600 \mathrm{MHz}, \mathrm{CDCl}_{3}\right)$.

correlations should be seen from the alkene proton to the allylic methylene group attached to the other alkene group (e.g. an H-12 correlation to H-14 and an H-13 correlation to H-11 should be seen for an $E$-geometry alkene). However, this 1D NOESY experiment was unsuccessful due to the close shifts of the two alkene protons making it difficult to irradiate one resonance without some irradiation of the other, this made it impossible to distinguish an nOe correlation between the alkene protons from the initial irradiation. The 1D NOESY was repeated in $\mathrm{C}_{6} \mathrm{D}_{6}$ (having previously been run in $\mathrm{CDCl}_{3}$ ), however this did not separate out the alkene shifts and the low solubility of dehydrohaliclocyclin $\mathrm{C}$ in $\mathrm{C}_{6} \mathrm{D}_{6}$ made the resolution of this experiment poor.

A 2D NOESY experiment was run to try and determine the geometry of the dehydrohaliclocyclin $\mathrm{C}$ alkene (Figure 4.7). This experiment appeared to show correlations from one alkene proton to the other (implying a $Z$-geometry, supporting the ${ }^{13} \mathrm{C}$ chemical shift argument), however, the correlations were weak and so close to the diagonal that this was not conclusive. Next, a fully coupled HSQC (which shows all couplings both hetero- and homonuclear to a proton and can be used to measure the coupling constants) was used to extract the coupling constants between the two alkene protons. For a $Z$-geometry the coupling between the alkene protons would be between 6 and $12 \mathrm{~Hz}$ (typically $10 \mathrm{~Hz}$ ) and for an $E$-geometry the coupling would be between 12 and $18 \mathrm{~Hz}$ (typically $17 \mathrm{~Hz}$ ). ${ }^{138}$ The coupled HSQC showed a coupling of $10 \mathrm{~Hz}$ between the major resonances (Figure 4.8), this provided further evidence for a $Z$-geometry. Finally, the most conclusive evidence for the Z-geometry of the double bond came from a homonuclear decoupling ${ }^{1} \mathrm{H} \mathrm{NMR}$ experiment (Figure 4.9). In this experiment, the allylic protons were irradiated (these proton resonances were conveniently overlapping in the ${ }^{1} \mathrm{H} \mathrm{NMR}$, allowing both to be irradiated at the same time), nullifying the coupling between the methylene and alkene 
protons. This experiment was able to resolve the alkene protons from multiplets into doublets and from these doublets a mutual coupling constant of $10.8 \mathrm{~Hz}$ was observed. This coupling constant is in the range of a $Z$-geometry alkene, confirming what had already been proposed from the ${ }^{13} \mathrm{C}$ NMR, 2D NOESY and coupled-HSQC data, that the alkene of dehydrohaliclocyclin $\mathrm{C}$ had a $Z$-geometry. This information, together with the singly charged molecular ion identified in the HRESIMS, led to the proposal of $\mathbf{5 8}$ as the structure for dehydrohaliclocyclin $\mathrm{C}$.

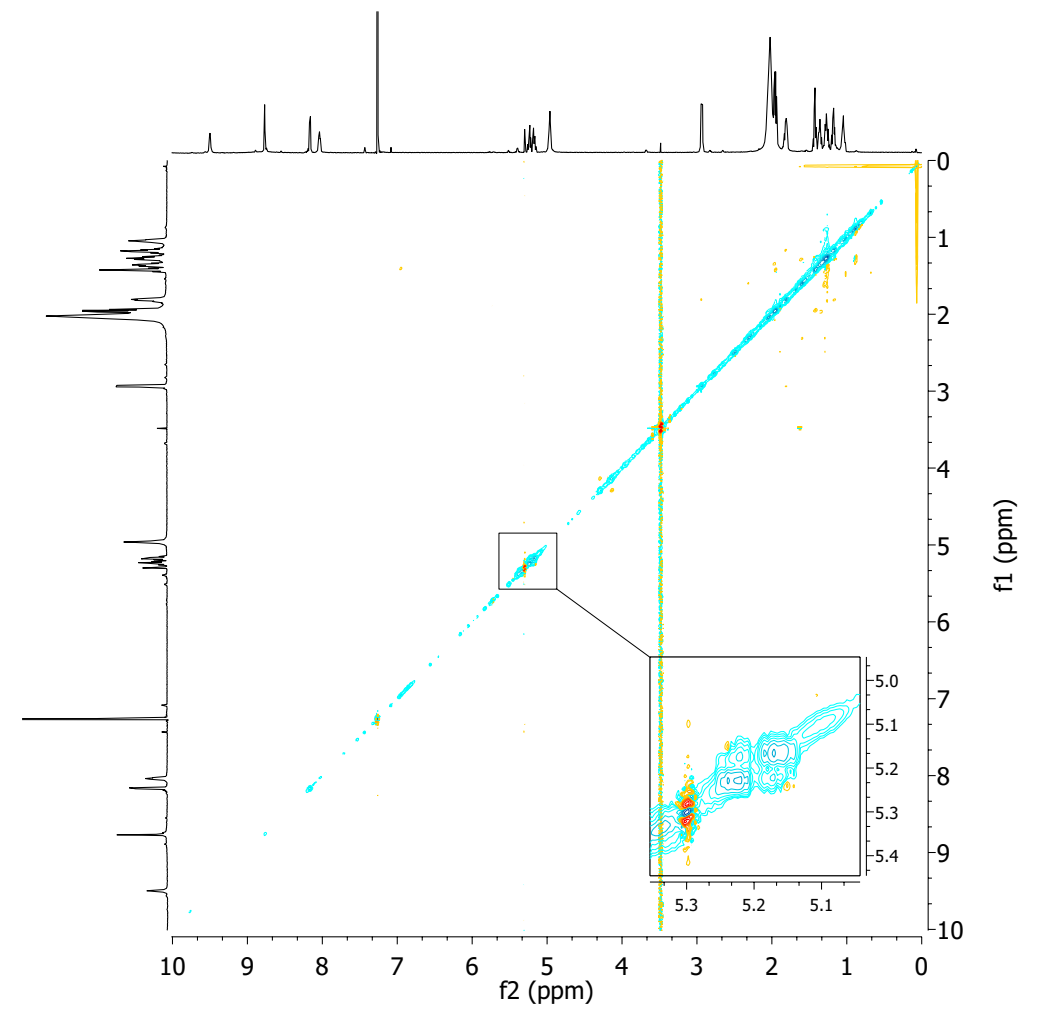

Figure 4.7. 2D NOESY NMR spectrum of dehydrohaliclocyclin $C$ with an expanded view of the weak alkene proton correlations $\left(600 \mathrm{MHz}, \mathrm{CDCl}_{3}\right)$.

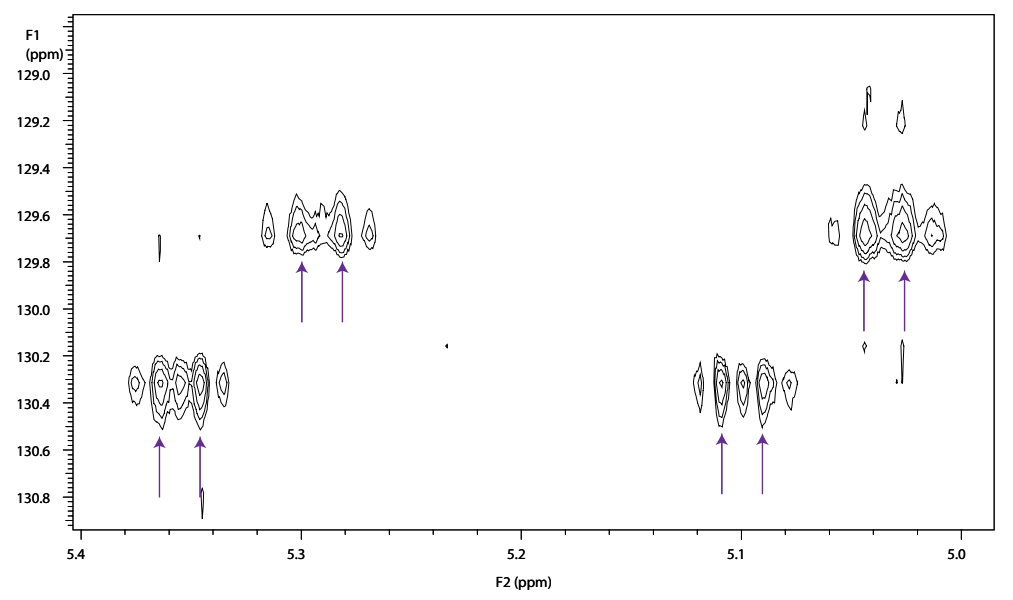

Figure 4.8. Expanded view of the alkene correlations in the coupled HSQC of dehydrohaliclocyclin $\mathrm{C}\left(600 \mathrm{MHz}, \mathrm{CDCl}_{3}\right)$. The measured splitting $(10 \mathrm{~Hz})$ from the coupling between the alkene protons is indicated (arrows). 

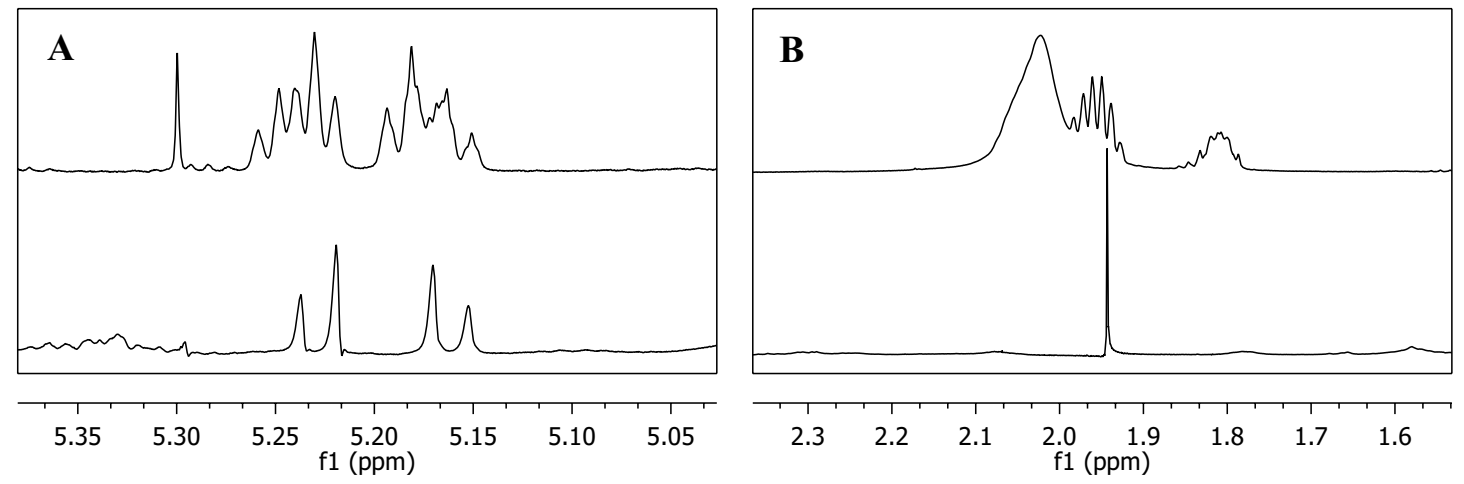

Figure 4.9. Homonuclear decoupled ${ }^{1} \mathrm{H}$ NMR spectrum of dehydrohaliclocyclin $\mathrm{C}(\mathbf{5 8})$ $\left(600 \mathrm{MHz}, \mathrm{CDCl}_{3}\right)$ showing an expanded view of the original (top) and decoupled (bottom) spectra highlighting alkene (A) and irradiated (B) resonances.

Table 4.5. ${ }^{13} \mathrm{C}(150 \mathrm{MHz})$ and ${ }^{1} \mathrm{H}(600 \mathrm{MHz}) \mathrm{NMR}$ data $\left(\mathrm{CDCl}_{3}\right)$ for dehydrohaliclocyclin C (58).

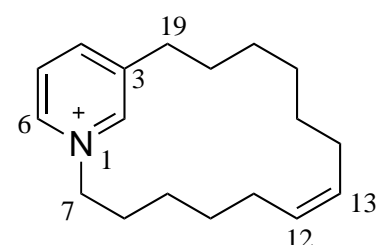

\section{8}

\begin{tabular}{|c|c|c|c|c|c|c|c|c|}
\hline \multirow[b]{2}{*}{ Position } & \multirow{2}{*}{$\frac{{ }^{13} \mathrm{C}}{\delta(\mathrm{ppm})}$} & \multirow[b]{2}{*}{${ }^{1} J_{\mathrm{CH}}(\mathrm{Hz})$} & \multicolumn{4}{|c|}{${ }^{1} \mathrm{H}$} & \multirow[b]{2}{*}{ COSY } & \multirow{2}{*}{$\begin{array}{c}\text { HMBC } \\
\left({ }^{1} \mathrm{H} \text { to }{ }^{13} \mathrm{C}\right)\end{array}$} \\
\hline & & & $\delta(\mathrm{ppm})$ & mult. & $J(\mathrm{~Hz})$ & int. & & \\
\hline 2 & 144.1 & 189 & 8.83 & $\mathrm{~s}$ & & 1 & $4,6,19$ & $3,4,7,19$ \\
\hline 3 & 143.4 & & & & & & & \\
\hline 4 & 145.2 & 171 & 8.17 & $\mathrm{~d}$ & 7.8 & 1 & $2,5,6,19$ & $2,5,6,19$ \\
\hline 5 & 128.5 & 171 & 8.03 & br t & 5.7 & 1 & 4,6 & 4,3 \\
\hline 6 & 143.6 & & 9.60 & brd & 5.3 & 1 & 2,5 & $2,4,5,7$ \\
\hline 7 & 62.7 & 150 & 5.01 & $\mathrm{~m}$ & & 2 & 8 & $2,6,8,9$ \\
\hline 8 & 31.1 & 124 & 2.06 & $\mathrm{~m}$ & & 2 & 7,9 & 7,9 \\
\hline 9 & 25.1 & 121 & 1.04 & $\mathrm{~m}$ & & 2 & 8,10 & $7,8,10,11$ \\
\hline 10 & 28.41 & 124 & 1.36 & $\mathrm{~m}$ & & 2 & 9,11 & $8,9,11,12$ \\
\hline 11 & 27.1 & 125 & 1.96 & $\mathrm{~m}$ & & 4 & 10,12 & $9,10,12,13$ \\
\hline 12 & 129.8 & 154 & 5.17 & $\mathrm{~m}$ & & 1 & 11,13 & $10,11,14$ \\
\hline 13 & 130.5 & 154 & 5.23 & $\mathrm{~m}$ & & 1 & 12,14 & $11,14,15$ \\
\hline 14 & 25.2 & 124 & 1.94 & $\mathrm{~m}$ & & 4 & 13,15 & $12,13,15,16$ \\
\hline 15 & 28.36 & 124 & 1.42 & quin & 6.5 & 2 & 14,16 & $13,14,16,17$ \\
\hline 16 & 27.3 & 118 & 1.27 & $\mathrm{~m}$ & & 2 & 15,17 & $14,15,17,18$ \\
\hline 17 & 26.6 & 122 & 1.17 & $\mathrm{~m}$ & & 2 & 16,18 & $15,16,18,19$ \\
\hline 18 & 29.0 & 127 & 1.81 & $\mathrm{~m}$ & & 2 & 17,19 & $3,16,17,19$ \\
\hline 19 & 32.3 & 130 & 2.94 & $\mathrm{~m}$ & & 2 & 18,2 & $2,3,4,17,18$ \\
\hline
\end{tabular}

\subsubsection{Dehydrohaliclocyclin F}

Dehydrohaliclocyclin F (59) was isolated as a colourless oil. Positive ion mode HRESIMS showed a singly charged molecular ion of $\mathrm{m} / z 272.2378(\mathrm{~m} / \mathrm{z} \Delta=0.0005)$ that indicated 


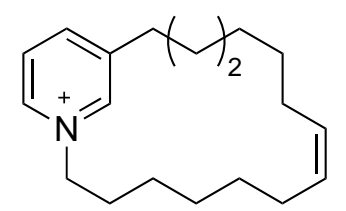

59

a molecular formula of $\mathrm{C}_{19} \mathrm{H}_{30} \mathrm{~N}^{+}$with an IHD of 5.5, indicating a natively charged structure with six double bonds and/or cyclic structures. The ${ }^{13} \mathrm{C}$ NMR spectrum was used in combination with the ${ }^{1} \mathrm{H}$ NMR and multiplicity-edited HSQC spectra to reveal four aromatic methines $\left(\delta_{\mathrm{C}} 147.1,145.8,143.3\right.$, and 129.4), one aromatic non-protonated carbon $\left(\delta_{\mathrm{C}} 145.3\right)$, two alkene methines $\left(\delta_{\mathrm{C}} 130.9\right.$ and 130.7), one deshielded methylene $\left(\delta_{\mathrm{C}} \quad 63.2\right)$ and 11 other methylene groups $\left(\delta_{\mathrm{C}} 33.3,31.6,30.8,30.2,30.1,29.1\right.$, $28.9,27.8,26.83,26.78,25.7)$. The resonances of protons attached to these carbons, determined using the multiplicity-edited HSQC spectrum, can be seen in Table 4.6. As for dehydrohaliclocyclin $\mathrm{C}$, the absence of any methyl groups in the ${ }^{13} \mathrm{C} \mathrm{NMR}$ and multiplicity-edited HSQC spectra indicated a cyclic structure.

The NMR spectra of dehydrohaliclocyclin F closely resembled the spectra of dehydrohaliclocyclin C. In the ${ }^{1} \mathrm{H}$ NMR spectrum there was a singlet at $8.95 \mathrm{ppm}(\mathrm{H}-2)$, a doublet at $8.87 \mathrm{ppm}(\mathrm{H}-6)$, another doublet at $8.51 \mathrm{ppm}(\mathrm{H}-4)$ and a triplet-like multiplet at $8.07 \mathrm{ppm}$ (H-5). This pattern of a singlet, doublet, doublet and triplet moving from downfield to upfield is typical of 3-APA structures. The ${ }^{1} \mathrm{H}$ NMR data, together with the five aromatic carbon resonances, clearly indicated that this was another 3-APA structure. Therefore, the HMBC spectrum could once again be used to determine the proton attachments at the 2-, 4- and 6-positions of the pyridinium ring. The proton singlet at $\delta_{\mathrm{H}} 8.95$ had HMBC correlations to the carbons at $\delta_{\mathrm{C}} 63.2$ and 33.3 (C-7 and C-20) and the carbon at $\delta_{\mathrm{C}} 145.3$ (C-3), indicating that this was the proton attached to the carbon at the 2-position. The proton doublet at $\delta_{\mathrm{H}} 8.87$ had an HMBC correlation to the C-7 carbon at $\delta_{\mathrm{C}} 63.2$ indicating that this proton was attached to the carbon at the 6-position in the pyridinium. Finally, the proton doublet at $\delta_{\mathrm{H}} 8.51$ had HMBC correlations to the C-20 carbon at $\delta_{\mathrm{C}} 33.3$ and the $\mathrm{C}-3$ carbon at $\delta_{\mathrm{C}} 145.3$, indicating that this proton was attached to the carbon at the 4-position of the pyridinium. This left the proton triplet at $\delta_{\mathrm{H}} 8.07$ to be in the 5-position of the pyridinium as expected from its upfield shift and multiplicity in the ${ }^{1} \mathrm{H}$ NMR spectrum (Figure 4.10).

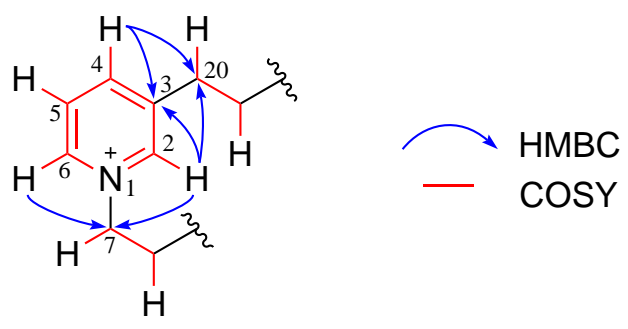

Figure 4.10. Key COSY and HMBC correlations establishing the 3-APA pyridinium substructure of dehydrohaliclocyclin F. 
Unlike the clearly resolved ${ }^{1} \mathrm{H}$ NMR spectrum of dehydrohaliclocyclin $\mathrm{C}$, the spectrum of dehydrohaliclocyclin $\mathrm{F}$ had a methylene envelope that is typical of long unsaturated alkyl chains (as is almost always seen in the ${ }^{1} \mathrm{H}$ NMR spectra of 3-APAs). This meant that the structure could not be solved so easily using the COSY spectrum as the protons next to functional groups (whose resonances had been shifted downfield in the ${ }^{1} \mathrm{H}$ NMR spectrum) would eventually show COSY correlations to the methylene envelope; this made it hard to determine the position of the double bond and the length of the alkyl chain. In the COSY spectrum, the protons in the 7-position at $4.68 \mathrm{ppm}$ (as shown from the HMBC and HSQC correlations) coupled to the protons at $2.03 \mathrm{ppm}(\mathrm{H}-8)$ that then coupled to the methylene envelope from 1.14 to $1.23 \mathrm{ppm}$ that further coupled to the other methylene envelope from 1.29 to 1.37 . The H-20 protons at $2.96 \mathrm{ppm}$ showed COSY correlations to the protons at $1.78 \mathrm{ppm}(\mathrm{H}-19)$ that further coupled to the methylene envelope from 1.14 to $1.23 \mathrm{ppm}$ as well. Finally, the overlapping alkene protons at 5.34 ppm showed COSY correlations to the protons at $1.89 \mathrm{ppm}$ that showed further correlations to the methylene envelope from 1.29 to $1.37 \mathrm{ppm}$. This spectral data gave the substructures shown in Figures 4.10 and 4.11 that were confirmed by HMBC correlations.

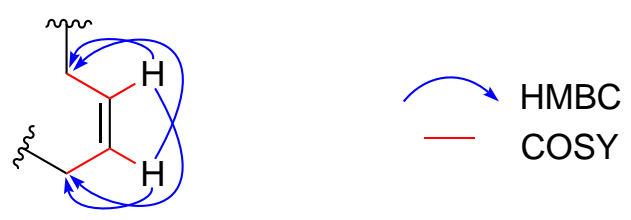

Figure 4.11. Key COSY and HMBC correlations of the alkene substructure of dehydrohaliclocyclin F.

1D TOCSY experiments were then used to prove that the 3-APA substructure and alkene substructure were connected (Figures 4.12, 4.13 and 4.14). Three of these experiments were performed, each one irradiating a different proton resonance of the compound. The 20-position protons at $\delta_{\mathrm{H}} 2.96$ (Figure 4.12) and the 7-position protons at $\delta_{\mathrm{H}} \quad 4.68$ (Figure 4.13) were irradiated to see if a connection to the alkene protons at $\delta_{\mathrm{H}} 5.34$ could be inferred. The alkene protons were also irradiated (Figure 4.14) to see if a connection to the 20- and 7-position protons could be inferred. The 1D TOCSY experiments were run using an array of mixing times from 15 to $150 \mathrm{~ms}$. This allowed the resonance of each proton down the alkyl chain from the irradiated protons to be seen sequentially until the weak resonances of the alkene protons at $\delta_{\mathrm{H}} 5.34$ (Figures 4.12 and 4.13) or the 20- and 7-position protons at $\delta_{\mathrm{H}} 2.96$ and 4.68 (Figure 4.14) were observed, proving that the two substructures were connected by a saturated alkyl chain.

Finally, the position and geometry of the alkene in dehydrohaliclocyclin $\mathrm{F}$ needed to be determined. As for dehydrohaliclocyclin $\mathrm{C}(\mathbf{5 8})$, a $Z$-geometry alkene was indicated by the ${ }^{13} \mathrm{C}$ chemical shifts of $\mathrm{C}-12$ and $\mathrm{C}-15$ (both $\delta_{\mathrm{C}}$ 26.8). A homonuclear decoupling experiment was also performed, with both sets of protons adjacent to the alkene being decoupled (Figure 4.15). The result of this experiment was not as clear as for dehydrohaliclocyclin $\mathrm{C}$, producing what is thought to be two overlapping doublet of 


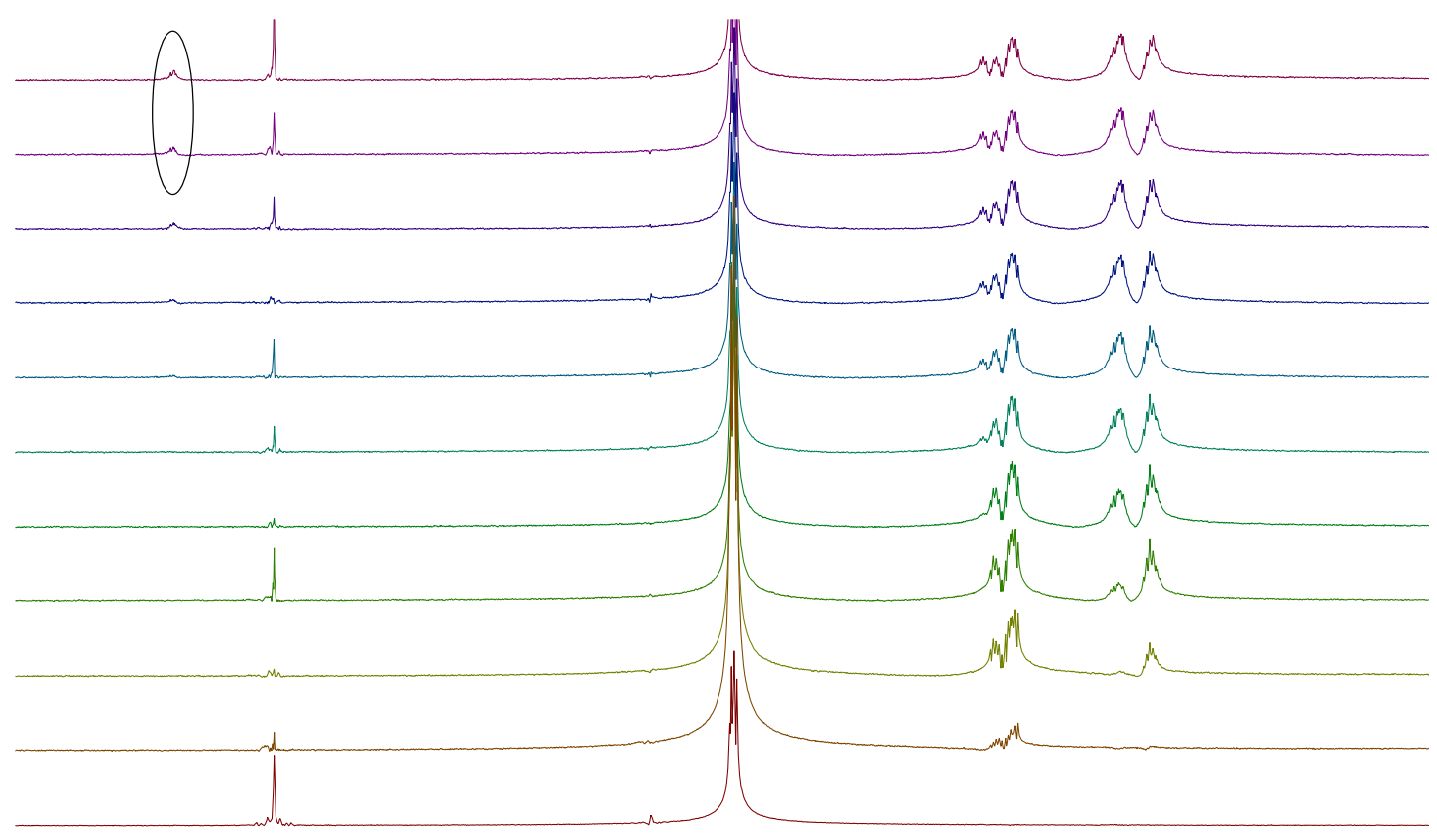

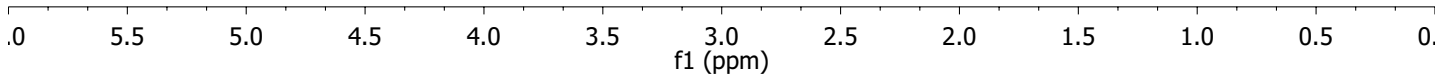

Figure 4.12. 1D TOCSY array irradiating the 20-position proton resonances (2.96 ppm) with mixing times from 15 (bottom) to 150 (top) milliseconds (600 MHz, $\mathrm{CD}_{3} \mathrm{OD}$ ). The alkene resonance at $5.34 \mathrm{ppm}$ is circled.

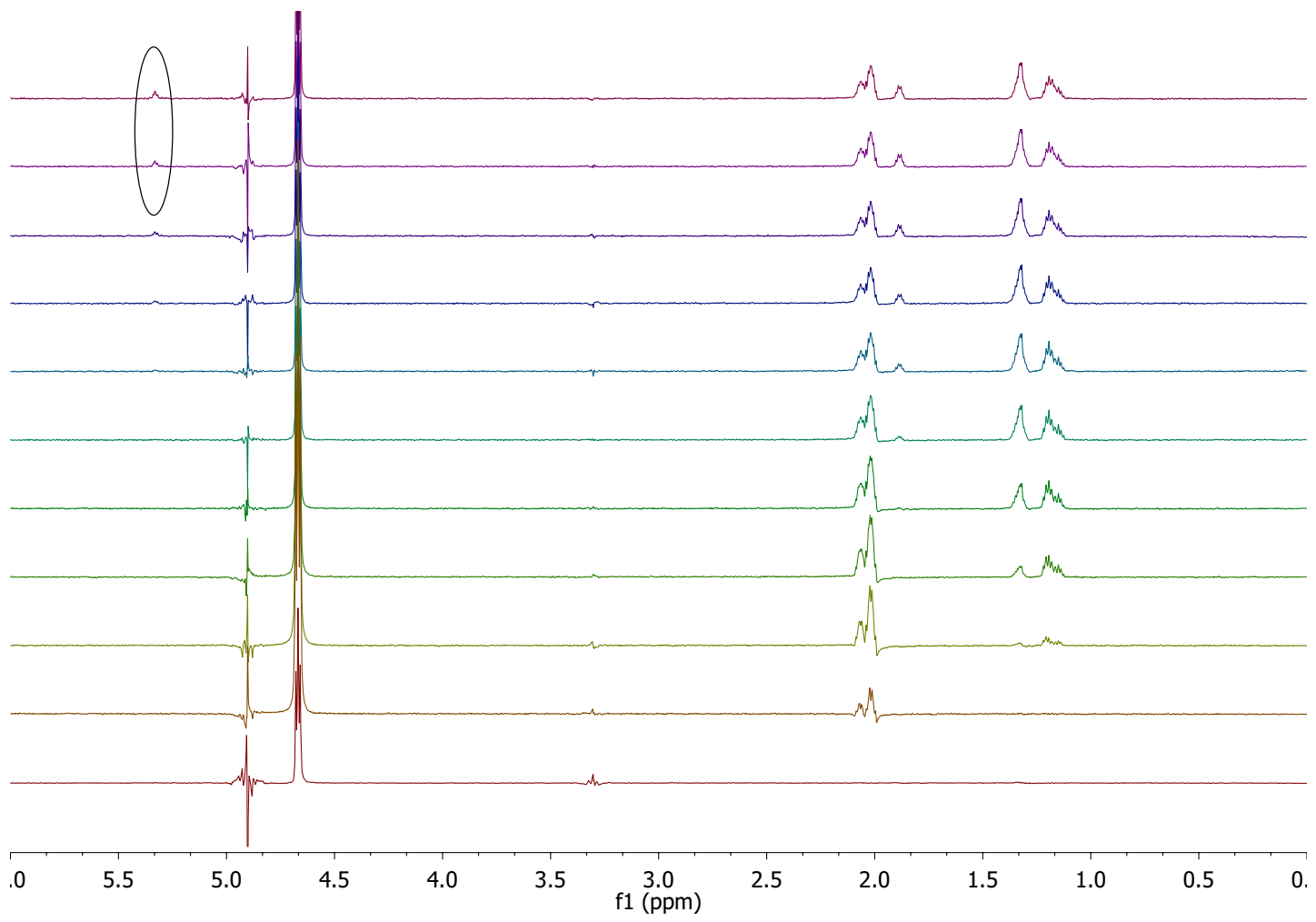

Figure 4.13. 1D TOCSY array irradiating the 7-position proton resonances (4.68 ppm) with mixing times from 15 (bottom) to 150 (top) milliseconds (600 MHz, $\mathrm{CD}_{3} \mathrm{OD}$ ). The alkene resonance at $5.34 \mathrm{ppm}$ is circled. 


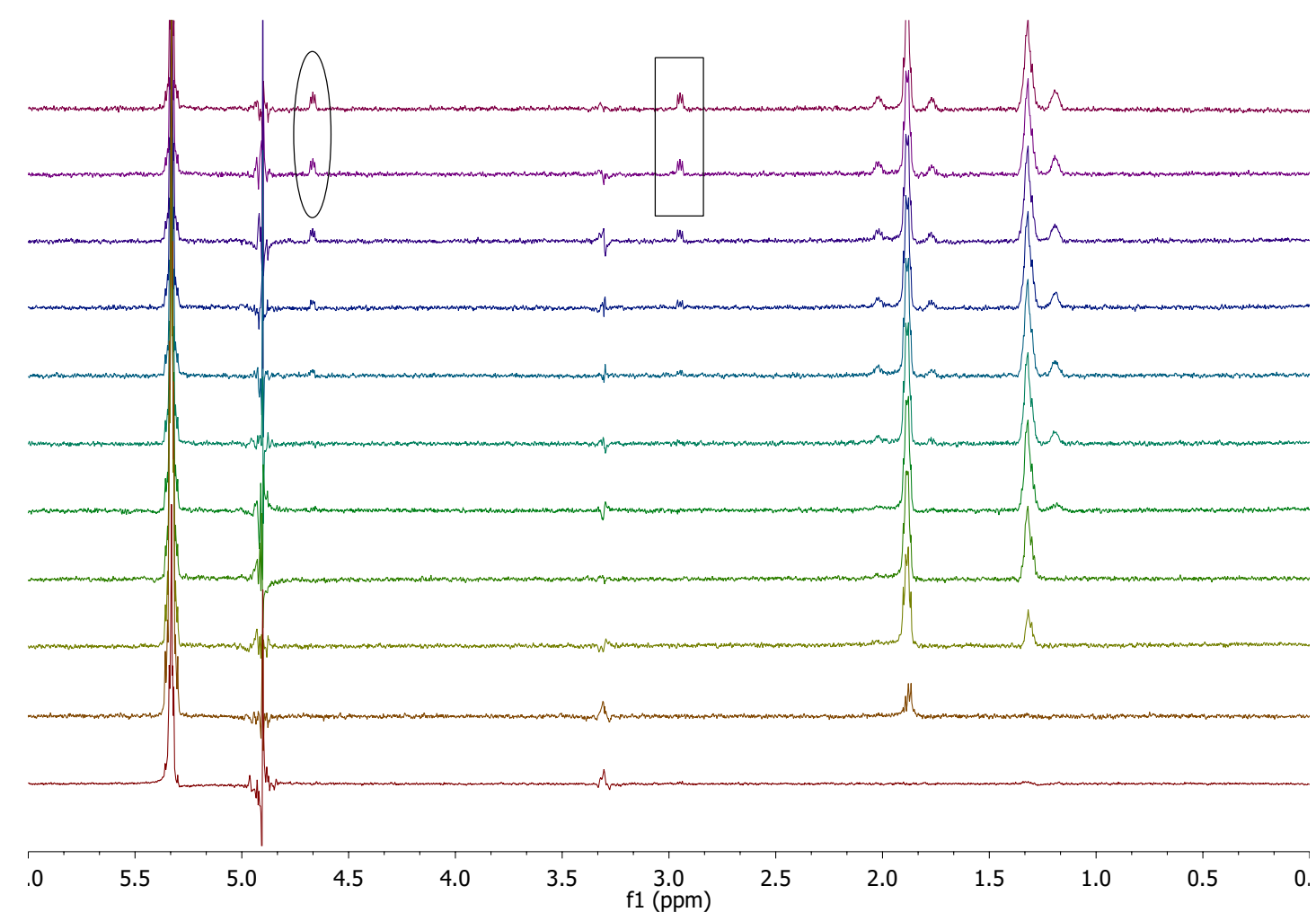

Figure 4.14. 1D TOCSY array irradiating the alkene proton resonances $(5.34 \mathrm{ppm})$ with mixing times from 15 (bottom) to 150 (top) milliseconds $\left(600 \mathrm{MHz}, \mathrm{CD}_{3} \mathrm{OD}\right.$ ). The $\mathrm{H}-7$ proton resonance at $4.68 \mathrm{ppm}$ is indicated by the circle and the $\mathrm{H}-20$ proton resonance at $2.96 \mathrm{ppm}$ is indicated by the square.

doublets from the highly second order multiplet. Despite this unclear resonance, the coupling that was measured between the very small outer peaks and inner peaks was between 10 and $11 \mathrm{~Hz}$ and could not be greater than $12 \mathrm{~Hz}$, this supported the proposal of a Z-geometry alkene. A fully coupled HSQC experiment was also performed and this showed that the coupling between the major resonances was $11.0 \mathrm{~Hz}$ (Figure 4.16), thus also indicating a $Z$-geometry for the alkene. With the evidence from these experiments a $Z$-geometry was proposed for the alkene in dehydrohaliclocyclin $\mathrm{F}$.
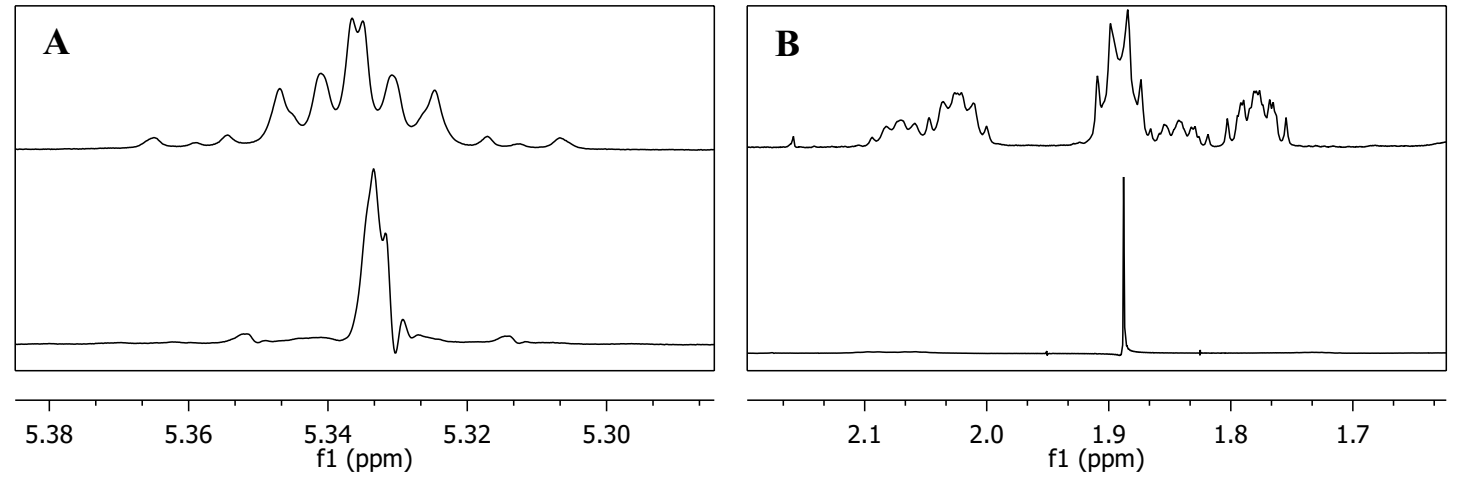

Figure 4.15. Homonuclear decoupled ${ }^{1} \mathrm{H}$ NMR spectrum of dehydrohaliclocyclin F (59) (600 MHz, $\mathrm{CD}_{3} \mathrm{OD}$ ) showing an expanded view of the original (top) and decoupled (bottom) spectra highlighting the alkene (A) and irradiated (B) resonances. 


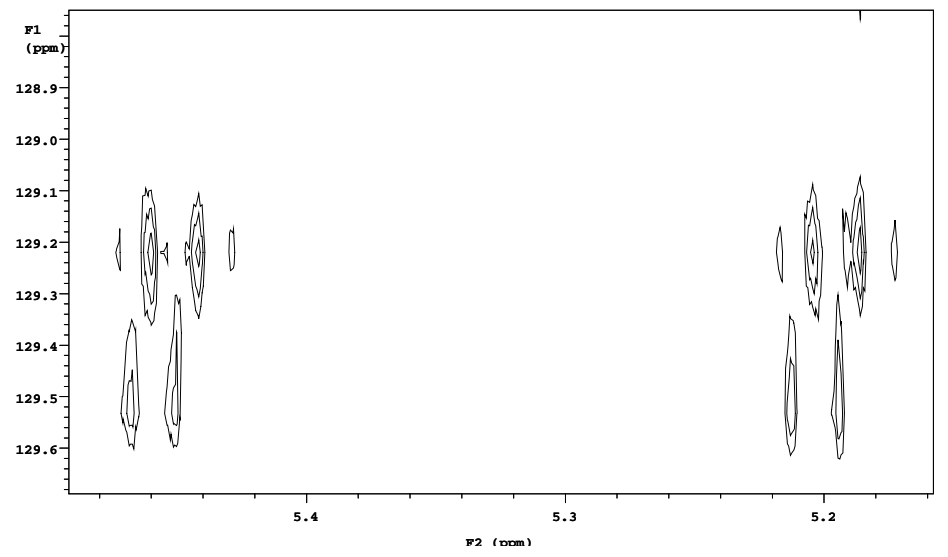

Figure 4.16. Expanded view of the alkene correlations in the coupled HSQC of dehydrohaliclocyclin $\mathrm{F}\left(600 \mathrm{MHz}, \mathrm{CD}_{3} \mathrm{OD}\right)$.

To determine the position of the double bond, an oxidation was performed using ruthenium trichloride and sodium periodate in a chloroform/acetonitrile/water solution. ${ }^{139-141}$ This successfully oxidatively cleaved the alkene to the dial (60) as shown by the MS peak detected at $\mathrm{m} / \mathrm{z}$ 304.2269. MS/MS analysis was then performed on the dialdehyde in order to identify the fragment peaks whose masses are determined by the alkyl chain lengths. The reproducible fragment mass of $m / z 192.1382$ was characteristic of a pyridinium with an alkyl chain length of seven, terminating with an aldehyde (61). This fragment is proposed to be the product of an onium reaction or the $[\mathrm{M}+\mathrm{H}]^{+}$of a Hofmann elimination on the dialdehyde, this would indicate that the alkene links carbons 13 and 14 of dehydrohaliclocyclin F.

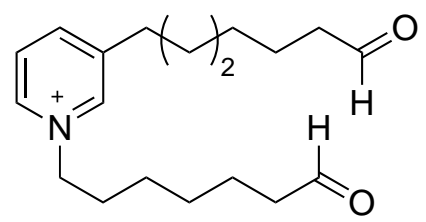

60
Onium Reaction

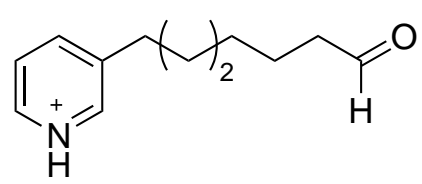

61 
Table 4.6. ${ }^{13} \mathrm{C}(150 \mathrm{MHz})$ and ${ }^{1} \mathrm{H}(600 \mathrm{MHz}) \mathrm{NMR}$ data $\left(\mathrm{CD}_{3} \mathrm{OD}\right)$ for dehydrohaliclocyclin $\mathrm{F}(\mathbf{5 9})$.

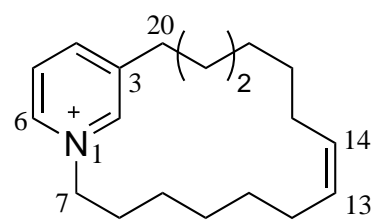

59

\begin{tabular}{|c|c|c|c|c|c|c|c|c|}
\hline \multirow[b]{2}{*}{ Position } & \multirow{2}{*}{$\frac{{ }^{13} \mathrm{C}}{\delta(\mathrm{ppm})}$} & \multirow[b]{2}{*}{${ }^{1} J_{\mathrm{CH}}(\mathrm{Hz})$} & \multicolumn{4}{|c|}{${ }^{1} \mathrm{H}$} & \multirow[b]{2}{*}{ COSY } & \multirow{2}{*}{$\begin{array}{c}\text { HMBC } \\
\left({ }^{1} \mathrm{H} \text { to }{ }^{13} \mathrm{C}\right)\end{array}$} \\
\hline & & & $\delta(\mathrm{ppm})$ & mult. & $J(\mathrm{~Hz})$ & int. & & \\
\hline 2 & 145.8 & 189 & 8.95 & $\mathrm{~s}$ & & 1 & 4,6 & $3,4,6,7,20$ \\
\hline 3 & 145.3 & & & & & & & \\
\hline 4 & 147.1 & 177 & 8.51 & d & 8.0 & 1 & $2,5,6$ & $2,3,6,20$ \\
\hline 5 & 129.4 & 176 & 8.07 & $\mathrm{~m}$ & & 1 & 4,6 & 3,6 \\
\hline 6 & 143.3 & 200 & 8.87 & d & 6.1 & 1 & $2,4,5$ & $2,4,5,7$ \\
\hline 7 & 63.2 & 147 & 4.68 & $\mathrm{t}$ & 6.1 & 2 & 8 & $2,6,8,9$ \\
\hline 8 & 31.6 & 123 & 2.02 & $\mathrm{~m}$ & & 2 & 7,9 & 7,9 \\
\hline $9^{a}$ & 25.7 & 116 & 1.20 & $\mathrm{~m}$ & & 4 & 8,10 & 10,11 \\
\hline $10^{b}$ & 30.2 & 122 & $(1.29-1.37)$ & $\mathrm{m}$ & & 8 & 9,11 & 9,11 \\
\hline $11^{c}$ & 29.1 & 123 & $(1.29-1.37)$ & $\mathrm{m}$ & & 8 & 10,12 & 9,10 \\
\hline $12^{d}$ & 26.83 & 123 & 1.89 & $\mathrm{~m}$ & & 4 & 11,13 & $10,11,13,14$ \\
\hline $13^{e}$ & 130.9 & 159 & 5.34 & $\mathrm{~m}$ & & 2 & 12 & $12 / 15$ \\
\hline $14^{e}$ & 130.7 & 159 & 5.34 & $\mathrm{~m}$ & & 2 & 15 & $12 / 15$ \\
\hline $15^{d}$ & 26.78 & 123 & 1.89 & $\mathrm{~m}$ & & 4 & 16,14 & $13,14,16,17$ \\
\hline $16^{c}$ & 28.9 & 123 & $(1.29-1.37)$ & $\mathrm{m}$ & & 8 & 17,15 & 17,18 \\
\hline $17^{b}$ & 30.1 & 122 & $(1.29-1.37)$ & $\mathrm{m}$ & & 8 & 16,18 & 16,18 \\
\hline $18^{a}$ & 27.8 & & 1.19 & $\mathrm{~m}$ & & 4 & 17,19 & $16,17,19$ \\
\hline 19 & 30.8 & & 1.78 & $\mathrm{~m}$ & & 2 & 18,20 & $3,18,20$ \\
\hline 20 & 33.3 & 130 & 2.96 & $\mathrm{~m}$ & & $2^{*}$ & 19 & $2,3,4,18,19$ \\
\hline
\end{tabular}

${ }^{a}$ Interchangeable

${ }^{b}$ Interchangeable

${ }^{c}$ Interchangeable

${ }^{d}$ Interchangeable

${ }^{e}$ Interchangeable

\subsubsection{Haliclocyclin C}

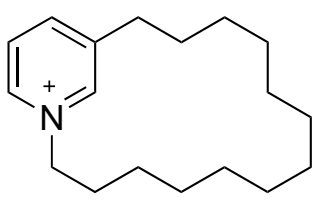

20

The compound haliclocyclin $\mathrm{C}(\mathbf{2 0})$ is known both naturally and synthetically (see Section 4.3.1). In the current research it was isolated as a colourless oil. Positive ion mode HRESIMS showed a singly charged molecular ion of $\mathrm{m} / \mathrm{z} 260.2381(\mathrm{~m} / \mathrm{z}, \Delta=0.0008)$ that indicated the molecular formula $\mathrm{C}_{18} \mathrm{H}_{30} \mathrm{~N}^{+}$with an IHD of 4.5, indicating a natively charged structure with five double bonds and/or cyclic structures. Only $0.16 \mathrm{mg}$ of this compound was isolated so the ${ }^{13} \mathrm{C}$, HSQC and HMBC NMR spectra were very weak, this meant that some resonances and correlations were not visible in these spectra. Some very weak correlations in the HSQC and HMBC spectra could only be found amongst the noise of the spectrum, these correlations were often still distinct from the surrounding noise, but could be easily missed if not specifically looking for them. Another concern was the 
contamination of this compound that occurred after quantitative ${ }^{1} \mathrm{H}$ NMR experiments that resulted in a monosubstituted benzene compound being present in this full set of spectra. These contamination correlations were luckily isolated and distinct from the haliclocyclin $\mathrm{C}$ correlations and so were ignored in the structural elucidation.

The ${ }^{13} \mathrm{C}$ NMR spectrum was used in combination with the ${ }^{1} \mathrm{H}$ NMR, multiplicity-edited HSQC and HMBC spectra to reveal the four aromatic methines $\left(\delta_{\mathrm{C}} 147.2,145.5,143.6\right.$, and 129.1), one aromatic non-protonated carbon $\left(\delta_{\mathrm{C}} 145.0\right)$, one deshielded methylene $\left(\delta_{\mathrm{C}}\right.$ 62.7) and 12 other methylene groups $\left(\delta_{\mathrm{C}} 32.6,31.3,30.1,28.1,28.0,27.94,27.90\right.$, $27.50,27.49,27.4,27.3,25.2)$. As for dehydrohaliclocyclin $\mathrm{C}$ and $\mathrm{F}$, the absence of any methyl groups in the ${ }^{13} \mathrm{C}$ NMR and multiplicity-edited HSQC spectra indicated a cyclic structure.

The proton resonances at $\delta_{\mathrm{H}} 8.97,8.86,8.50$ and 8.06 in the singlet, doublet, doublet and doublet of doublets pattern from downfield to upfield, were characteristic of a 3-APA. As expected, the singlet resonance at $8.97 \mathrm{ppm}(\mathrm{H}-2)$ in the ${ }^{1} \mathrm{H}$ NMR spectrum had HMBC correlations to the 7 and 19-position carbons ( $\delta_{\mathrm{C}} 62.7$ and 32.6, respectively) indicating that it was the proton attached to the carbon at the 2-position of the pyridinium. The doublet at $8.86 \mathrm{ppm}$ (H-6) had an HMBC correlation to the 7-position carbon indicating that it was the proton attached to the carbon in the 6-position. The doublet at $8.50 \mathrm{ppm}$ (H-4) had an HMBC correlation to the 19-position carbon, indicating that this was the proton attached to the carbon at the 4-position and this left the doublet of doublets at $8.06 \mathrm{ppm}$ as the proton attached to the carbon in the 5-position of the pyridinium (Figure 4.17).

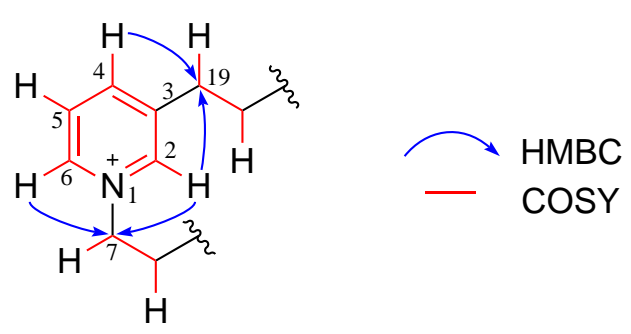

Figure 4.17. Key COSY and HMBC correlations establishing the 3-APA pyridinium substructure of haliclocyclin C (20).

As for the compounds dehydrohaliclocyclin $\mathrm{C}$ and $\mathrm{F}$, the carbon attached to the nitrogen at the 7-position and its protons had characteristic chemical shifts $\left(\delta_{\mathrm{C}} 62.7\right.$ and $\left.\delta_{\mathrm{H}} 4.67\right)$ and could be unambiguously identified using the HMBC and multiplicity-edited HSQC spectra. The COSY spectrum showed correlations from these protons at $4.67 \mathrm{ppm}(\mathrm{H}-7)$ to the protons at $2.07 \mathrm{ppm}(\mathrm{H}-8)$ that showed further correlations to the methylene envelope between 1.14 and $1.19 \mathrm{ppm}$ that showed correlations to the other methylene envelope between 1.27 and $1.36 \mathrm{ppm}$. The carbon and attached protons at the 19-position could similarly be identified using the HMBC correlations in combination with the multiplicityedited HSQC ( $\delta_{\mathrm{C}} 32.6$ and $\delta_{\mathrm{H}}$ 2.96). The COSY spectrum showed correlations from the 
protons at $2.96 \mathrm{ppm}(\mathrm{H}-19)$ to the protons at $1.84 \mathrm{ppm}(\mathrm{H}-18)$ that had further correlations to the methylene envelope between 1.14 and $1.19 \mathrm{ppm}$ as well. This gave the typical 3-APA substructure shown in Figure 4.17. The lack of any unsaturation in the alkyl chain of this 3-APA (as shown by no major resonances in the ${ }^{1} \mathrm{H}$ NMR spectrum around $5.3 \mathrm{ppm}$ ) meant that the alkyl chain length could only be solved using MS. The HRESIMS peak of $m / z 260.2381$, therefore, established the structure of this compound as the known compound haliclocyclin C (20). All spectroscopic data were consistent with that reported in the literature. ${ }^{113,114,116}$

\subsubsection{VD4_47A}

VD4_47A was isolated as a yellow oil. The ${ }^{13} \mathrm{C}$ NMR spectrum of this sample was used in combination with the ${ }^{1} \mathrm{H}$ NMR and multiplicity-edited HSQC spectra to reveal four aromatic methines $\left(\delta_{\mathrm{C}} 146.7,145.3,143.4\right.$, and 129.0), one aromatic non-protonated carbon $\left(\delta_{\mathrm{C}}\right.$ 145.7), one deshielded methylene $\left(\delta_{\mathrm{C}}\right.$ 62.9) and several other methylene groups that were indistinguishable from minor component resonances. Some of these resonances observed in the ${ }^{13} \mathrm{C}$ NMR spectrum were broad, indicating that this sample was not purely one 3-APA. The methylene resonances that were distinguishable from the overlapping minor resonances and proposed to be part of the major component due to their intensity and characteristic 3-APA shifts were $\delta_{\mathrm{C}} 33.5,32.5$ and 31.6. The ${ }^{13} \mathrm{C}$ NMR spectrum also contained six alkene methines $\left(\delta_{\mathrm{C}} 131.3,131.2,130.9,130.7,130.3,130.2\right)$ that all showed a single combined correlation to a proton multiplet between 5.31 and $5.41 \mathrm{ppm}$ in the HSQC spectrum.

From these correlations it was unclear whether the alkenes were part of the major 3-APA as the resonances all had lower intensities in the ${ }^{13} \mathrm{C}$ NMR spectrum and they showed a 1:1 integration ratio with the aromatic protons in the ${ }^{1} \mathrm{H}$ NMR spectrum (indicating there is only one alkenyl proton for every pyridinium group in the structure). The HMBC spectrum could not be used to clarify this as the alkene proton correlations to an impurity structure could not be differentiated from correlations to the major compound. From this information it was proposed that the alkene is not part of the major 3-APA since it would be expected that the proton multiplet of the alkene would have a greater than 1:1 ratio with the aromatic protons, since it is obviously the overlapping resonance of several alkenes as shown by the ${ }^{1} \mathrm{H},{ }^{13} \mathrm{C}$ and multiplicity-edited HSQC NMR spectra. There is a possibility that this integration ratio is due to a double bond being present in just one alkyl chain of a dimer (as in seen in the dehydrocyclostellettamines 29, 30, 35 and 46-51). However, this would indicate that the impurity also had the same motif due to the integration ratio being 1:1 despite the overlapping resonances. As for dehydrohaliclocyclin $\mathrm{C}$ and $\mathrm{F}$ and haliclocyclin $\mathrm{C}$, the absence of any methyl groups in the ${ }^{13} \mathrm{C}$ NMR and multiplicity-edited HSQC spectra indicated a cyclic structure. 
As for the previous compounds, the HMBC spectrum could be used to confirm that the proton singlet resonance at $\delta_{\mathrm{H}} 8.98$ was of the proton attached to the carbon in the 2-position, the doublet resonance at $\delta_{\mathrm{H}} 8.87$ was of the proton attached to the carbon in the 6-position, the doublet resonance at $\delta_{\mathrm{H}} 8.47$ was of the proton attached to the carbon in the 4-position and the triplet resonance at $\delta_{\mathrm{H}} 8.03$ was of the proton attached to the carbon in the 5-position. The COSY spectrum could be used to show that the 7-position protons at $4.63 \mathrm{ppm}$ coupled to the protons between 2.00 and $2.04 \mathrm{ppm}$ that then coupled to the methylene envelope between 1.27 and $1.39 \mathrm{ppm}$. The COSY spectrum also showed that the protons attached to the methylene carbon that was adjacent to the 3-position of the pyridinium, at $2.89 \mathrm{ppm}$, coupled to the protons at $1.73 \mathrm{ppm}$ that further coupled to the methylene envelope. Further information to delineate the structure could not be obtained, however, it is proposed that further purification of the sample is required before a final structure can be identified. This work was not able to be completed in the current study due to time constraints.

\subsubsection{Biological Activity}

The 3-APAs isolated were originally identified by testing for the inhibition of S. cerevisiae growth. They showed potent activity in initial assays, as described in Section 4.4. However, upon the isolation of the pure 3-APA compounds, there was not enough mass to test for activity against both $S$. cerevisiae and $C$. albicans. It is for this reason that the inhibitory activity of the pure 3-APA monomers was not measured against $S$. cerevisiae in favour of more clinically relevant biological evaluation. Unfortunately, dehydrohaliclocyclin C (58), dehydrohaliclocyclin F (59) and haliclocyclin C (20) showed no activity against $C$. albicans when tested at $0.2,2$ and $20 \mu \mathrm{g} / \mathrm{mL}$ concentrations in a disc diffusion assay. Possible reasons for this observation are that the samples tested were too dilute, the 3-APAs had poor diffusion through the agar medium or that these 3-APA monomers are simply inactive against $C$. albicans. 


\section{Chapter 5}

\section{Concluding Remarks}

The marine environment is a rich source of interesting and unusual compounds that have potent biological activities. This makes it the ideal environment to explore in the search for new biologically active compounds to be used as potential drug leads. In the current study two new and three known compounds have been isolated using a bioassay and NMR-guided approach. Crude extract libraries of New Zealand and Tongan marine invertebrates were screened against $S$. cerevisiae and 'hits' from this were validated. Three New Zealand sponges and one Tongan ascidian were identified as having inhibitory activity towards the growth of $S$. cerevisiae. These invertebrates were extracted and chromatographic methods were used to purify bioactive and structurally interesting compounds as shown by the bioassays and ${ }^{1} \mathrm{H}$ NMR spectra used to guide the isolation process.

The known trihydroxylated steroid (14) was isolated from a calcareous sponge that has been tentatively identified as Leucosolenia sp. The fractionation of this sponge used a bioassay-guided approach, however, poor activity was observed. A mixture of trihydroxylated steroids that differed in the side chain were isolated and this was purified further to allow the structure of one steroid to be solved as a representative of the mixture. The mass of steroid $\mathbf{1 4}$ isolated, in combination with the poor activity observed and time constraints, meant that it was not submitted for biological testing. A fatty acid and mixture of phthalate esters were isolated from an unidentified Tongan ascidian. The fatty acid was tentatively assigned as cis-vaccenic acid (13) based on the comparison of ${ }^{1} \mathrm{H}$ and ${ }^{13} \mathrm{C} \mathrm{NMR}$ spectra. These compounds were not pursued further in the interest of time, due to a lack of activity and the well-known nature of these compound classes.

An assay that targets bacterial MscL was also being developed in this study for its use at VUW to test against the extract library of New Zealand and Tongan marine invertebrates. Some parameters were able to be established for use in this assay and others were proposed but further work had to be done to confirm them. Unfortunately, the liposomes 
that were prepared for this assay were fragile and yielded inconsistent results in both the optimisation of the parameters and attempts at screening the extract library, resulting in no 'hits' being identified. It is possible that there were no compounds present in the extract library screened that interact with the very specific MscL target, however, this cannot be concluded with certainty without a clearer distinction between MscL active and inactive compounds being observed in the assay. More work needs to be done to understand the effect of each aspect of the liposome preparation on the stability of the liposomes so that more robust liposomes can be made. Once the liposome preparation has been optimised the ideal parameters to be used in the assay can be better determined to allow for clear distinctions between positive 'hits' and negative controls. This would allow the assay to be more effective in identifying bioactive natural products as molecular probes for Msc.

One known and two new 3-APAs were isolated from a New Zealand sponge identified as Haliclona sp. Initially, this was done using a bioassay-guided approach that allowed 3-APAs to be identified as the active components and, following this, an NMR-guided approach was used to isolate the pure compounds. A range of biological activities have been reported for 3-APAs, including antibacterial, antifungal and cytotoxic activity. The known 3-APA monomer isolated, haliclocyclin C (20), was previously reported to show potent antibacterial, neurotoxic and cytotoxic activity, however, when tested against C. albicans in the present study, no activity was found. The new 3-APA monomers dehydrohaliclocyclin $C$ (58) and dehydrohaliclocyclin F (59) are the first examples of 3-APA cyclic monomers with unsaturation in the alkyl chain. Their structures were elucidated using a combination of NMR spectroscopy, MS and an oxidative alkene cleavage for dehydrohaliclocyclin F. When tested for inhibition of $C$. albicans growth, dehydrohaliclocyclins $\mathrm{C}$ and $\mathrm{F}$ showed no activity at the concentrations tested. It is possible that the lack of activity that was observed for these compounds was due to the samples tested being too dilute, poor diffusion of the 3-APAs in agar or the monomers isolated were simply inactive against $C$. albicans.

Future work on this project would involve further purifying the sample VD4_47A so that the 3-APAs present can be identified and fully characterised. It would also involve purifying other fractions isolated from Haliclona sp. that contained 3-APAs in order to identify the 3-APAs present (for example the 60\% acetone in water fraction from the initial HP20 column). It would also be useful to extract and purify more Haliclona sp. to isolate more of the compounds dehydrohaliclocyclin $\mathrm{C}$ and $\mathrm{F}$ for more biological testing as well as to identify and characterise other 3-APAs present. Alternatively, a synthetic approach could be taken to collecting more of the compounds dehydrohaliclocyclin $\mathrm{C}$ and $\mathrm{F}$ for more biological testing. It would also be interesting to extract and purify more Leucosolenia sp. in order to isolate more trihydroxylated steroids so that more of the structures could be fully elucidated and the steroids isolated could be submitted to biological testing. Finally, the liposome preparation in the MscL assay will need to be optimised in future studies so that more stable and reproducible liposomes are available 
for use in the assay. This can be done by adjusting aspects such as the amount of drying time given to remove all of the chloroform from the azolectin film, the amount of time the solution is sonicated for in creating the liposomes, the amount of MscL added to liposome solutions and the amount of time the liposome solutions spend mixing with Biobeads ${ }^{\circledR}$. The possibility of leaving the liposome solution mixing with Biobeads ${ }^{\circledR}$ overnight should also be explored as this would allow more experiments to be run with one batch of liposomes in a day. 


\section{Chapter 6}

\section{Experimental}

\subsection{General Experimental}

Marine invertebrates were collected from various locations in New Zealand and Tonga by the Marine Natural Products group at VUW. These sponges were stored in a freezer at $-20{ }^{\circ} \mathrm{C}$ until required. Crude extracts from New Zealand and Tongan marine invertebrates that were tested in assays had been sampled into $3 \times 96$-well plates. These master plates were then subsampled into daughter plates with approximately $50 \mu \mathrm{g}$ of extract in each well and these were stored in the fridge until required.

Normal-phase column chromatography was performed using Supelco Discovery ${ }^{\circledR}$ DSCDIOL functionalised silica: 3-(2,3-Dihydroxy-propoxy)-propyl-silica (Diol). Reversedphase column chromatography was performed using Supelco Diaion ${ }^{\circledR}$ HP20 and HP20ss poly(styrene-divinylbenzene) (PSDVB) resins. Two separate HPLC systems were used, the first using a Rainin Dynamax SD-200 solvent delivery system with $25 \mathrm{~mL}$ pump heads and a Varian Prostar 335 photodiode array detector for UV/Vis detection. The second HPLC system was an Agilent Technologies 1260 Infinity HPLC equipped with a quaternary pump, a thermostatted column compartment and diode array detector (DAD). Following the DAD, a Quicksplit ${ }^{\mathrm{TM}}$ flow splitter directs $5 \%$ of the flow to an Agilent 380 -evaporative light scattering detector (ELSD) with $95 \%$ of the flow directed towards collection. For HPLC purification a Phenomenex analytical $(5.0 \times 250 \mathrm{~mm}, 5 \mu \mathrm{m}$ particle size) custom-packed Diol column was used for normal-phase. For reversed-phase, C18 analytical (Phenomenex Prodigy, $4.6 \times 250 \mathrm{~mm}, 5 \mu \mathrm{m}$ particle size) or semi-preparative (Phenomenex Prodigy, $10.0 \times 250 \mathrm{~mm}, 10 \mu \mathrm{m}$ particle size) columns were used.

All solvents used were HPLC-grade (purchased from Fisher Scientific) with the exception of hexanes and dichloromethane, which were Optima ${ }^{\circledR}$ grade. Water was distilled prior to use and in the case of HPLC the water was further filtered through a membrane with a pore size of $0.45 \mu \mathrm{m}$ before use. The solvent compositions used are all reported as 
the $\%$ v/v. TLC analyses were performed using Machery-Nagel Polygram ${ }^{\circledR}$ Sil G/UV 254 plates and visualised under UV light ( $\lambda=254 \mathrm{~nm}$ and $350 \mathrm{~nm})$. TLC plates were then visualised by dipping in $5 \%$ conc. $\mathrm{H}_{2} \mathrm{SO}_{4}$ in methanol (v/v\%) followed by $0.1 \%$ vanillin in ethanol $(\mathrm{w} / \mathrm{v} \%)$ and heated for analysis.

NMR spectra were obtained using a Varian DirectDrive spectrometer equipped with a triple resonance $\mathrm{HCN}$ cryogenic probe operating at $600 \mathrm{MHz}$ for ${ }^{1} \mathrm{H}$ and $150 \mathrm{MHz}$ for ${ }^{13} \mathrm{C}$ nuclei. The chemical shifts of ${ }^{1} \mathrm{H}$ and ${ }^{13} \mathrm{C}$ spectra were referenced to the residual solvent peaks $\left(\mathrm{CDCl}_{3}: \delta_{\mathrm{C}}\right.$ 77.0, $\delta_{\mathrm{H}}$ 7.26; $\mathrm{CD}_{3} \mathrm{OD}: \delta_{\mathrm{C}}$ 49.0, $\delta_{\mathrm{H}}$ 3.31). All NMR solvents were purchased from Aldrich with the exception of deuterated methanol, which was purchased from Cambridge Isotope Laboratories Inc. NMR quantification of final compounds were performed with an internal nitromethane $\left(\mathrm{CH}_{3} \mathrm{NO}_{2}\right)$ standard and using the acquisition parameters described by West. ${ }^{142}$ HRESIMS results were obtained from an Agilent 6530 Q-TOF mass spectrometer equipped with an Agilent 1260 HPLC for solvent delivery utilising a JetStream ${ }^{\mathrm{TM}}$ electrospray ionisation source in positive and negative ion modes. Water and acetonitrile $(\mathrm{MeCN})$ solvents were both made up as $5 \mathrm{mM}$ solutions with ammonium formate. CID was performed using nitrogen as collision gas at various energies (10-100, arbitrary units). Where a column was used it was a C18 Zorbax Extend $(2.1 \times 50 \mathrm{~mm}, 1.8 \mu \mathrm{m}$ particle size $)$. A typical MS run using a column started at $5 \% \mathrm{MeCN}$ for 0.5 minutes followed by a 3.5 minute gradient up to $100 \% \mathrm{MeCN}$, the solvent composition was held at $100 \% \mathrm{MeCN}$ for 0.5 minutes and then there was a 0.05 minute gradient back down to $5 \% \mathrm{MeCN}$, the composition was then held at $5 \%$ $\mathrm{MeCN}$ for 2.45 minutes.

For sponge identification, the organic matter was dissolved by immersing small samples $\left(\leq 1 \mathrm{~cm}^{3}\right.$ ) in concentrated $\mathrm{HNO}_{3}$ or $12.5 \% \mathrm{NaOCl}$ (for calcareous sponges). Any spicules that remained after this were then washed by suspending them in water and then centrifuging them to allow the water to be decanted, this process was repeated several times. The spicules were then imaged using an Olympus ${ }^{\circledR}$ Research Microscope AX70 Provis equipped with an Olympus ${ }^{\circledR}$ DP70 Digital Microscope Camera and these images were compared with descriptions in the literature to classify the sponge.

\subsection{Saccharomyces cerevisiae Assay}

The Saccharomyces cerevisiae yeast strains BY4742 (wild type) and yCG 117 were used in this work. yCG 117 was used to screen the crude extracts initially and after extraction BY4742 was used to guide the fractionation. Both of these strains were streaked out from a $15 \%$ glycerol stock that was stored at $-80^{\circ} \mathrm{C}$ onto $10 \mathrm{~cm}$ agar plates containing either synthetic complete (SC) media or yeast extract peptone dextrose (YPD) media. These plates were then incubated at $30^{\circ} \mathrm{C}$ for 48 hours, allowing single yeast colonies to form, 
the plates were then stored at $4{ }^{\circ} \mathrm{C}$. Single colonies from these plates were then used in making up liquid culture for bioassays as required. Cycloheximide was used as a positive control in all of the $S$. cerevisiae assays. Cell growth in assays was quantified using optical density (OD) measurements acquired on a Perkin Elmer Wallac EnVision 2102 Multilabel Plate Reader. Plates were vortexed using an Eppendorf MixMate plate mixer.

Table 6.1. Yeast Strains Used

\begin{tabular}{|c|c|c|c|}
\hline Strain & Ploidy & Genotype & Origin \\
\hline yCG 117 & haploid & 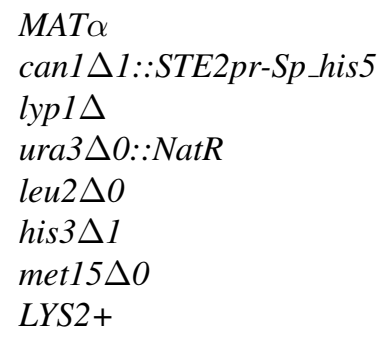 & Starting lab strain \\
\hline BY4742 & haploid & $\begin{array}{l}\text { MATQ } \\
\text { his } 3 \Delta l \\
\text { leu } 2 \Delta O \\
\text { lys } 2 \Delta O \\
\text { ura } 3 \Delta O\end{array}$ & Starting lab strain \\
\hline
\end{tabular}

\subsubsection{Extract Library Screening Protocol}

For screening the extract library against yeast a $100 \mu \mathrm{g} / \mathrm{mL}$ concentration of crude extract was used. In order to do this, $20 \mu \mathrm{L}$ of 1:1 DMSO to water was added to each well of the daughter plates that contained approximately $50 \mu \mathrm{g}$ of the crude extract and the plates were vortexed. This gave a crude extract concentration of $2.5 \mathrm{mg} / \mathrm{mL}$ and from this $4 \mu \mathrm{L}$ from each extract well was added to $96 \mu \mathrm{L}$ of yeast culture to give an extract concentration of $100 \mu \mathrm{g} / \mathrm{mL}$ in each well.

The yeast culture was made up using a single colony of yeast from an agar plate added to $5 \mathrm{~mL}$ of SC liquid media and incubated overnight at $30^{\circ} \mathrm{C}$ on a shaker to saturation. A $10 \mu \mathrm{L}$ aliquot of this culture was then diluted by adding it to $990 \mu \mathrm{L}$ of water and vortexing before placing $10 \mu \mathrm{L}$ of this diluted culture on to a microscope slide grid to count. The cells on the grid were counted using a haemocytometer and from this the concentration of the saturated yeast culture could be calculated. The saturated yeast culture was then diluted to a concentration of $5 \times 10^{5}$ cells $/ \mathrm{mL}$ using fresh SC media and this was vortexed. The 96-well plates for the assay were then set up using $96 \mu \mathrm{L}$ of this SC media plus cells mixture and $4 \mu \mathrm{L}$ of extract in 1:1 DMSO to water was added to this. A positive control, solvent control, cell blank and media blank were also used in each plate of the assay in order to determine the growth (measured by OD) of cells with and without solvent, the 
OD of cells that have not grown and the OD and sterility of the media. The positive control was cycloheximide and $0.3 \mu \mathrm{L}$ of a $1 \mathrm{mM}$ solution in DMSO was added to four wells containing the media plus cells mixture. The solvent control was $4 \mu \mathrm{L}$ of $1: 1 \mathrm{DMSO}$ to water and this was also added to four wells of the media plus cells mixture. The cell blank and media blank were four wells each of plain media plus cells mixture and plain media, respectively. The wells were mixed by vortexing the plates at 950 RPM for one minute and these plates were then incubated at $30^{\circ} \mathrm{C}$ for 18 hours.

When incubation was complete the yeast cells were resuspended by vortexing the plate at 950 RPM for one minute and the growth was then measured using OD at $590 \mathrm{~nm}$. OD effects of the media were then subtracted from the remaining wells by subtracting the optical density of the average of the media blank from the optical density of the yeast growth wells to get a normalised value. The residual growth was then calculated by dividing the normalised growth of the experimental wells by the normalised growth of the solvent control wells and multiplying by 100 to give percentage growth

$\left(\left(\mathrm{OD}_{\text {exp norm. }} / \mathrm{OD}_{\text {solv. norm. }}\right) \times 100\right)$. From this a percentage inhibition could be calculated by subtracting the percentage growth from 100. A 'hit' was considered to be a minimum of $35 \%$ growth inhibition of $S$. cerevisiae. This data was processed using Microsoft Excel 2010.

\subsubsection{Dose-Response Assay Protocol}

'Hits' were validated using a dose-response assay. The validations were performed using subsamples of crude extracts that had been stored in vials in the fridge. The highest concentration used in the validation assays was $100 \mu \mathrm{g} / \mathrm{mL}$ and serial dilutions were performed in blank media. Subsequent assays used to guide the isolation of natural products used the same protocol as the validation assays, with the exception that the initial concentrations used in the dose-response curves were adjusted according to the potency of the extracts or fractions.

For dose-response assays yeast cultures were made two-fold higher in cell concentration $\left(10 \times 10^{5}\right.$ cells $\left./ \mathrm{mL}\right)$ using the same protocols used for extract library screening. Serial dilutions of extracts were made in media and yeast culture was then added to the extract/media mixture. The dilutions were performed by placing $92 \mu \mathrm{L}$ of media in column two of a 96-well plate, and $50 \mu \mathrm{L}$ of media in columns 3-11 (columns one and 12 and rows $\mathrm{A}$ and $\mathrm{H}$ were used for controls). To column two, $8 \mu \mathrm{L}$ of extract in a 1:1 DMSO to water solution, at a concentration of $2.5 \mathrm{mg} / \mathrm{mL}$ was added to the wells in triplicate. This resulted in a concentration of $200 \mu \mathrm{g} / \mathrm{mL}$ in the column two wells. Taking $50 \mu \mathrm{L}$ from these wells, two-fold dilutions were carried out in subsequent columns until column 11. Following the serial dilutions, $50 \mu \mathrm{L}$ of yeast cell culture was added to the extract/media wells, resulting in final extract concentrations of $100 \mu \mathrm{g} / \mathrm{mL}$ through to 


\section{$0.2 \mu \mathrm{g} / \mathrm{mL}$.}

The dose-response assay plates were mixed and incubated using the same protocol used for the screening assay. The same controls were also used, this time with the solvent and positive controls being performed as dose-response curves alongside the experimental wells. Percentage growth and inhibition were calculated from these results using Microsoft Excel 2010. IC $_{50}$ values reported for this data were determined using a non-linear regression on GraphPad Prism version 5.00 where the concentration values had been transformed to a logarithmic scale. ${ }^{143}$

\subsection{Candida albicans assay}

The purified samples of dehydrohaliclocyclin $\mathrm{C}(0.36 \mathrm{mg})$, dehydrohaliclocyclin $\mathrm{F}$ $(0.30 \mathrm{mg})$ and haliclocyclin $\mathrm{C}(0.16 \mathrm{mg})$ had $9.0,7.5$ and $4.0 \mathrm{~mL}$ of methanol added to them, respectively, to make up a concentration of $40 \mu \mathrm{g} / \mathrm{mL}$. These stock solutions were then diluted to $0.2,2$ and $20 \mu \mathrm{g} / \mathrm{mL}$ concentrations and $10 \mu \mathrm{L}$ aliquots of these dilutions were spotted on to sterile filter paper discs. These discs were placed on malt yeast agar (MYA) plates that had been inoculated with a culture of $C$. albicans. As a positive control, $10 \mu \mathrm{g}$ of rapamycin was spotted on to a disk and as a negative control, sterile water was spotted on to a disk. The plates were incubated for 24 hours at $37^{\circ} \mathrm{C}$ and zones of inhibition were measured. This work was performed by Jason Ryan at Callaghan Innovation.

\subsection{MscL Assay}

Azolectin, N-2-Hydroxyethylpiperazine-N'-2-ethanesulfonic acid (HEPES) and Triton ${ }^{\mathrm{TM}}$ X-100 were purchased from Sigma, 5,6-carboxyfluorescein (CF) was purchased from Fluka and n-dodecyl- $\beta$-D-maltopyranoside (DDM, analytical grade) was purchased from Affymetrix Anatrace. Avestin polycarbonate membranes with $400 \mathrm{~nm}$ pore diameters were purchased from ATA Scientific, BioBeads ${ }^{\circledR}$ SM-2 adsorbent were purchased from Bio-Rad and 1-oleoyl-2-hydroxy-sn-glycero-3-phosphocholine (LPC) was purchased from Avanti ${ }^{\circledR}$ Polar Lipids, Inc. These were kindly supplied to us by Prof. Boris Martinac at the Victor Chang Cardiac Research Institute via Dr. Penny Truman at ESR. Also supplied was an Avestin LiposoFast-Basic lipid extruder with a LiposoFast-Stabilizer used to give uniform liposome sizes. As well as the MscL protein suspended in DDM buffer at a concentration of $394 \mu \mathrm{g} / \mathrm{mL}$ and a control DDM buffer. The DDM buffer had been made up using $1 \mathrm{mM}$ DDM in phosphate buffered saline (PBS). 
The chloroform used in preparing the liposomes was HPLC-grade and purchased from Panreac. Water was distilled and deionised using a Milli-Q water filtration system. HEPES buffer was made up to $50 \mathrm{mM}$ HEPES in water where the $\mathrm{pH}$ was adjusted to 7.2-7.4 using $\mathrm{KOH}$, this was stored in the fridge. DR buffer was made up as required using one part $1 \mathrm{M} \mathrm{KCl}$, one part $50 \mathrm{mM}$ HEPES buffer and eight parts water. The CF buffer was made up using a $50 \mathrm{mM}$ concentration of CF in DR buffer, adjusting the $\mathrm{pH}$ to 7.2 using $5 \mathrm{M} \mathrm{KOH}$. The CF buffer was stored in the fridge. The LPC was made up using $100 \mathrm{mg}$ of LPC dissolved in $2 \mathrm{~mL}$ of methanol $(0.1 \mathrm{M})$. This was later diluted to a concentration of $0.05 \mathrm{M}$ by adding an equal volume of methanol to the amount remaining in the vial. The LPC in methanol solution was stored in the freezer at $-20{ }^{\circ} \mathrm{C}$. A $10 \%$ Triton $^{\mathrm{TM}}$ solution was made up using approximately $1 \mathrm{~mL}$ of $\operatorname{Triton}^{\mathrm{TM}} \mathrm{X}-100$ dissolved in $9 \mathrm{~mL}$ of water. Gel filtration of the liposomes was performed using Sephadex G-25 medium in Bio-Rad disposable columns, these were equilibrated in water and stored in the fridge.

To detect the liposomes in this assay a Perkin Elmer EnSpire ${ }^{\mathrm{TM}} 2300$ Multilabel Plate Reader with quad-monochromator based fluorescence intensity detection was used. All MscL assays were run in 96-well black plates.

\subsubsection{Assay Protocol}

The protocol used for the MscL assay was modified from a protocol provided by Dr. Penny Truman (ESR) in order to create MscL-containing liposomes at VUW. To make the liposomes, $20 \mathrm{mg}$ of azolectin was dissolved in $2 \mathrm{~mL}$ of chloroform in a small sample vial and this was then dried using a rotary evaporator to leave a thin film of azolectin in the vial. Once the sample was dry it was placed on the freeze drier for 10 to 15 minutes to remove any residual chloroform. To this azolectin film, $1 \mathrm{~mL}$ of CF buffer was added and the solution was vortexed for one minute then sonicated in an ice water bath for 20 minutes. The solution was then passed through a membrane with a pore size of $400 \mathrm{~nm}$ using a lipid extruder. The liposome solution was then divided into two eppendorfs, $300 \mu \mathrm{L}$ of the solution was placed in each eppendorf and $10 \mu \mathrm{L}$ of MscL in DDM buffer was added to one and $10 \mu \mathrm{L}$ of DDM buffer to the other. The eppendorfs were then inverted by rotation at 20 RPM, to mix the solutions, for one hour.

After mixing for one hour, a volume of BioBeads ${ }^{\circledR}$ (washed three times with methanol and three times with water), approximately one third the volume of the liposome solutions, was added to the eppendorfs. The solutions were then mixed again for two to four hours. After mixing, the liposomes were separated from unincorporated CF buffer using gel filtration in a column that had been equilibrated in DR buffer. The liposomes were eluted from the column using DR buffer and were easily identifiable as the fast moving fluorescent material in the column. The liposomes collected from this were then ready to 
be diluted and used in an assay.

Liposomes were diluted to three different concentrations using DR buffer (usually $\frac{1}{5}, \frac{1}{10}$ and $\frac{1}{20}$ dilutions) and tested with $98 \mu \mathrm{L}$ of liposome dilution and $2 \mu \mathrm{L}$ of DR buffer or $10 \%$ Triton $^{\mathrm{TM}}$ in triplicate to read the fluorescence of intact and burst liposomes. From these readings dilutions were adjusted to achieve the desired fluorescence. Assays were performed using the buffer, solvent, LPC and Triton ${ }^{\mathrm{TM}}$ controls tested against both MscL and control liposomes in triplicate. The wells were made up using $98 \mu \mathrm{L}$ of the liposome solution with $2 \mu \mathrm{L}$ of control (DR buffer, methanol, $10 \%$ Triton $^{\mathrm{TM}}$ ) or test extract, with the exception of LPC, which was added in amounts varying from $0.5 \mu \mathrm{L}$ to $2 \mu \mathrm{L}$ at the concentrations of $0.1 \mathrm{M}$ and $0.05 \mathrm{M}$ as specified in the assay description. Using liposome solutions in DR buffer, dose-response assays were set up in a similar fashion to the $S$. cerevisiae assays, replacing the solvent 1:1 DMSO in water with methanol.

Assays were read at 5 or 10 minute intervals after LPC addition for up to 30-60 minutes. The fluorescence percentage $\left(\mathrm{F}_{\%}\right)$ was calculated from the average fluorescence of the controls or tests $\left(\mathrm{F}_{\text {avg. }}\right)$ divided by the average fluorescence of the Triton ${ }^{\mathrm{TM}}$ controls $\left(\mathrm{F}_{\text {T.avg. }}\right)$ and multiplying the result by $100\left(\mathrm{~F}_{\%}=\left(\mathrm{F}_{\text {avg. }} /\left(\mathrm{F}_{\text {T.avg. }}\right) \times 100\right)\right.$. These calculations were only used with the controls from the same liposome types (e.g. MscL liposomes fluorescence were compared to MscL liposomes Triton ${ }^{\mathrm{TM}}$ control fluorescence only). The percentage fluorescence could then be compared between the control $\left(\mathrm{F}_{\% C}\right)$ and $\mathrm{MscL}$ $\left(\mathrm{F}_{\% M}\right)$ liposomes by subtracting the percentage fluorescence of the control liposomes from the percentage fluorescence of the $\mathrm{MscL}$ liposomes to give a fluorescence percentage difference, $\mathrm{D}_{\%}\left(\mathrm{D}_{\%}=\mathrm{F}_{\% M}-\mathrm{F}_{\% C}\right)$. A difference in percentage fluorescences of the MscL and control solvent controls ( $\mathrm{D}_{\% \text { Solv. }}$ ) could also be calculated and this could then be subtracted from the differences in the percentage fluorescences of the LPC-containing test or extract test wells $\left(\mathrm{D}_{\% L P C}\right)$, this would allow the data to be corrected for the relative fragility of the MscL and control liposomes ( $\mathrm{D}_{\% c o r r .}=\mathrm{D}_{\% L P C}-\mathrm{D}_{\% \text { Solv. }}$ ). Data processing was done using Microsoft Excel 2010.

\subsection{Isolation of 3-APAs from MNP_0999}

Frozen Haliclona sp. (MNP_0999, 189 g) was extracted twice in methanol (250 mL). These extracts were then filtered through a pad of celite to remove any solid material. The methanol extracts were cyclic loaded onto $75 \mathrm{~mL}$ of cleaned HP20 resin with the second extract being run through the column followed by the first extract. The eluent from this was collected together and $500 \mathrm{~mL}$ of water was added to it to make a $50 \%$ methanol solution. This solution was then passed through the column again and $1 \mathrm{~L}$ of water was added to the eluent to make a $25 \%$ methanol solution. This $25 \%$ methanol solution was then passed through the column. The column, which was now loaded with the MNP_0999 
extract, was then eluted using $225 \mathrm{~mL}$ of $0 \%, 20 \%, 40 \%, 60 \%, 80 \%$ and $100 \%$ acetone in water.

The $20 \%, 40 \%, 60 \%$ and $80 \%$ acetone in water fractions were each backloaded on to $25 \mathrm{~mL}$ of cleaned HP20 resin in order to remove the water. To do this, the fractions were diluted with a further $225 \mathrm{~mL}$ of water and run through the column slowly to load the extract fraction onto the column. The column was then eluted with $75 \mathrm{~mL}$ of methanol followed by $75 \mathrm{~mL}$ of acetone, these elutions were collected together and the solvent removed in vacuo. The $100 \%$ acetone fraction was also dried in vacuo. The $20 \%, 40 \%$, $60 \%, 80 \%$ and $100 \%$ fractions were labelled VD1_96A, B, C, D and E, respectively. These fractions were transferred to pre-weighed sample vials using methanol (VD1_96A, $\mathrm{B}$ and $\mathrm{C}$ ) or $20 \% \mathrm{CH}_{2} \mathrm{Cl}_{2}$ in methanol (VD1_96D and E). The fractions were dried in vacuo (VD1_96A and B) or under a stream of compressed air (VD1_96C, D and E) to recover masses of $127.9 \mathrm{mg}$ (VD1_96A), 388.4 mg (VD1_96B), 447.5 mg (VD1_96C), $160.8 \mathrm{mg}$ (VD1_96D) and $91.3 \mathrm{mg}$ (VD1_96E).

These fractions were then tested against $S$. cerevisiae in a dose-response assay testing a maximum concentration of $200 \mu \mathrm{g} / \mathrm{mL}$. This assay gave approximate $\mathrm{IC}_{50}$ values of $1.5 \pm 1.0 \mu \mathrm{g} / \mathrm{mL}$ for VD1_96A and $\mathrm{B}$, an $\mathrm{IC}_{50}$ of $1.5 \mu \mathrm{g} / \mathrm{mL}$ with a very large margin of error for VD1_96C, an $\mathrm{IC}_{50}$ of $10.2 \pm 1 \mu \mathrm{g} / \mathrm{mL}$ for VD1_96D and showed no inhibition for VD1_96E. ' ${ }^{1} \mathrm{H}$ NMR spectra were also collected for all of these fractions and they showed that VD1_96A, B and C all contained similar compounds (3-APAs) whereas VD1_96D and E did not. Fractions VD4_96A and B were purified further.

\subsubsection{Dehydrohaliclocyclin C and F and Haliclocyclin C}

Fraction VD1_96A was further fractionated using $30 \mathrm{~mL}$ of cleaned HP20ss resin and eluted with $100 \mathrm{~mL}$ of $5 \%, 10 \%, 20 \%, 30 \%, 50 \%$ and $100 \%$ methanol in water. This produced samples VD2_373A-I and ${ }^{1} \mathrm{H}$ NMR were run on these. This fractionation was NMR-guided in order to identify the compounds producing interesting multiplet resonances occurring around 5.1 and $5.2 \mathrm{ppm}$ in the ${ }^{1} \mathrm{H}$ NMR spectrum. Fraction VD4_373B, which was isolated from the $50 \%$ methanol in water elution, showed the resonances of interest in the ${ }^{1} \mathrm{H}$ NMR spectrum and had a mass of $24.3 \mathrm{mg}$. Further purification of VD2_373B was done using a $16 \mathrm{~mL}$ Diol column, eluting with $50 \mathrm{~mL}$ of $100 \%, 50 \%$ and $0 \%$ hexanes in ethyl acetate followed by $25 \%, 50 \%, 75 \%$ and $100 \%$ methanol in ethyl acetate, finally the column was stripped with $25 \%$ and $50 \%$ water in methanol. This produced fractions VD2_384A-O, the ${ }^{1} \mathrm{H}$ NMR of these showed that 3-APAs were present in fractions VD2_384G-N. The multiplet resonances of interest were present in fractions VD2_384G-I and of these VD2_384G had the highest mass of $6.7 \mathrm{mg}$. 
Further purification of VD2_384G was performed using reversed-phase (C18) analytical HPLC, eluting with acetonitrile in water (both with $0.1 \%$ formic acid) with a flow rate of $1 \mathrm{~mL} / \mathrm{min}$. This was performed on the Rainin HPLC system. The method held the solvent mixture at $25 \%$ acetonitrile for four minutes followed by an eight minute ramp to $50 \%$ acetonitrile, holding at $50 \%$ for five minutes, there was then a two minute ramp to $100 \%$ acetonitrile, a hold at $100 \%$ for five minutes followed by a two minute ramp back down to $25 \%$ acetonitrile and finally the solvent mixture was held at $25 \%$ acetonitrile for six minutes (32 minutes total). The elution profile was followed using the UV/Vis trace at 210 and $270 \mathrm{~nm}$ and fractions VD2_394A-K were collected. The fractions of interest were VD2 394F and G, which had strong, sharp peaks in the UV/Vis trace and so were expected to be the 3-APAs, this was confirmed using ${ }^{1} \mathrm{H}$ NMR. Fraction VD2_394F was sufficiently pure that a full set of NMR data could be run on this, resulting in the identification of dehydrohaliclocyclin C (58). A full set of NMR data was also run of VD2_394G revealing a mixture of 3-APAs. Both VD2_394F and G were further purified using HPLC.

The same HPLC conditions used in the purification of VD2_384G were used for the purification of VD2_394F, with the exception that the hold at 50\% acetonitrile in water for five minutes in the run was shortened to a three minute hold, shortening the run time to 30 minutes. The UV/Vis trace of this showed one strong peak (VD3_86B) and one weaker peak (VD3_86D). Fraction VD3_86B (0.8 mg) was the compound dehydrohaliclocyclin $\mathrm{C}$ as shown by the ${ }^{1} \mathrm{H}$ NMR spectrum. 1D and 2D NOESY, coupled-HSQC and homonuclear decoupling NMR experiments were all run on this compound allowing its geometry to be assigned. Over the course of these experiments the sample accumulated some impurity and so needed to be purified again. This time the purification was done on the Agilent HPLC system using a reversed-phase (C18) analytical column and eluting with methanol in water (both with $0.1 \%$ formic acid) and with a flow rate of $1 \mathrm{~mL} / \mathrm{min}$. The purification used an isocratic 10 minute run of $53 \%$ methanol in water and the elution profile was followed using the UV/Vis trace at 210 and $270 \mathrm{~nm}$. There was only one strong, major peak in the UV/Vis trace and from this the fraction VD4_33C was isolated, its mass was then measured using quantitative NMR and it was submitted for biological analysis against $C$. albicans. Fraction VD4_33C (0.36 mg) was the pure compound dehydrohaliclocyclin C (58).

Fraction VD2_394G was purified using the same HPLC conditions used to purify VD2_384G. The UV/Vis trace showed one major peak that had two shoulders, the peak was collected as two fractions in order to aid separation of the compounds. The first part of the major peak (VD3_87C) showed a cleaner ${ }^{1} \mathrm{H}$ NMR spectrum as well as appearing to have a larger mass in the ${ }^{1} \mathrm{H}$ NMR than the second part of the major peak (VD3_87D). A full set of NMR experiments were run on VD3_87C and these revealed two 3-APAs, which MS data suggested were dehydrohaliclocyclin F (59) and haliclocyclin C (20). To separate out these two compounds, reversed-phase (C18) analytical HPLC was used, eluting with 
methanol in water (both with $0.1 \%$ formic acid) and using a flow rate of $1 \mathrm{~mL} / \mathrm{min}$. This HPLC purification was performed in two halves. The first half of purification used the Rainin HPLC and collected fractions VD4_27A-E and the second half of the purification used the Agilent HPLC and the fractions VD4_31A-D were collected. A 15 minute isocratic run of $53 \%$ methanol in water was used in both separations. ${ }^{1} \mathrm{H}$ NMR spectra showed that VD4_27C and VD4_31C were both the same compound and that VD4_27B and VD4_31B were both the same compound. The matching compounds were recombined and VD4_27 and 31C were renamed to VD4_32A and VD4_27 and 31B were renamed to VD4_32B. The masses of these compounds were measured using quantitative NMR and they were then submitted for biological analysis against $C$. albicans. VD4_32A $(0.25 \mathrm{mg})$ was the new compound dehydrohaliclocyclin F (59) and VD4_32B (0.16 mg) was the known compound haliclocyclin $\mathrm{C}(\mathbf{2 0})$.

Dehydrohaliclocyclin C (58): Colourless oil, $0.36 \mathrm{mg} ;{ }^{1} \mathrm{H} \mathrm{NMR}\left(\mathrm{CDCl}_{3}, 600 \mathrm{MHz}\right)$ $\delta 9.60$ (br d, $J=5.3 \mathrm{~Hz}, 1 \mathrm{H}, \mathrm{H}-6$ ), 8.83 (s, 1H, H-2), 8.17 (d, J=7.8 Hz, 1H, H-4), 8.03 (br t, J=5.7 Hz, 1H, H-5), 5.23 (m, 1H, H-13), 5.17 (m, 1H, H-12), 5.01 (m, 2H, H-7), 2.94 (m, 2H, H-19), 2.06 (br m, 2H, H-8), 1.98-1.93 (m, 4H, H-11 \& H-14), 1.81 (m, 2H, H-18), 1.42 (quin, J=6.5 Hz, 2H, H-15), 1.36 (m, 2H, H-10), 1.27 (m, 2H, H-16), 1.17 (m, 2H, H-17), 1.04 (m, 2H, H-9); ${ }^{13} \mathrm{C} \mathrm{NMR}\left(\mathrm{CDCl}_{3}, 150 \mathrm{MHz}\right) \delta 145.2(\mathrm{C}-4)$, 144.1 (C-2), 143.6 (C-6), 143.4 (C-3), 130.5 (C-13), 129.8 (C-12), 128.5 (C-5), 62.7 (C-7), 32.3 (C-19), 31.1 (C-8), 29.0 (C-18), 28.41 (C-10), 28.36 (C-15), 27.3 (C-16), 27.1 (C-11), 26.6 (C-17), 25.2 (C-14), 25.1 (C-9); Further NMR data presented in Table 4.5; HRESIMS $[\mathrm{M}]^{+} m / z 258.2216$ for $\mathrm{C}_{18} \mathrm{H}_{28} \mathrm{~N}^{+}$(calculated 258.2216, $m / z \Delta=0$ ), $[\mathrm{M}+1]^{+}$ $\mathrm{m} / \mathrm{z} 259.2250$ (calculated 259.2249, $\mathrm{m} / \mathrm{z}, \Delta=0.0001$ ).

Dehydrohaliclocyclin F (59): Colourless oil, $0.25 \mathrm{mg} ;{ }^{1} \mathrm{H} \mathrm{NMR}\left(\mathrm{CD}_{3} \mathrm{OD}, 600 \mathrm{MHz}\right)$ $\delta 8.95$ (s, 1H, H-2), 8.87 (d, J=6.1 Hz, 1H, H-6), 8.51 (d, J=8.0 Hz, 1H, H-4), 8.07 (m, 1H, H-5), 5.35 (m, 2H, H-13 \& H-14), 4.68 (t, J=6.1 Hz, 2H, H-7), 2.96 (m, 2H, H-20), 2.02 (m, 2H, H-8), 1.89 (m, 4H, H-12 \& H-15), 1.78 (m, 2H, H-19), 1.37-1.29 (m, 8H, H-10, H-11, H-16 \& H-17), 1.23-1.14 (m, 4H, H-9 \& H-18); ${ }^{13} \mathrm{C}$ NMR $\left(\mathrm{CD}_{3} \mathrm{OD}, 150 \mathrm{MHz}\right) \delta 147.1$ (C-4), 145.8 (C-2), 145.3 (C-3), 143.3 (C-6), 130.9 (C-13/14), 130.7 (C-13/14), 129.4 (C-5), 63.2 (C-7), 33.3 (C-20), 31.6 (C-8), 30.8 (C-19), 30.2 (C-10/17), 30.1 (C-10/17), 29.1 (C-11/16), 28.9 (C-11/16), 26.83 (C-12/15), 26.78 (C-12/15), 27.8 (C-18), 25.7 (C-9); Further NMR data presented in Table 4.6; HRESIMS [M] ${ }^{+} \mathrm{m} / \mathrm{z}, 272.2378$ for $\mathrm{C}_{19} \mathrm{H}_{30} \mathrm{~N}^{+}$(calculated 272.2373, $\mathrm{m} / \mathrm{z}, \Delta=0.0005$ ), $[\mathrm{M}+1]^{+} \mathrm{m} / \mathrm{z} 273.2412$ (calculated 273.2406, $\left.\mathrm{m} / \mathrm{z} \Delta=0.0006\right),[\mathrm{M}+2]^{+} \mathrm{m} / \mathrm{z} 274.2443$ (calculated 274.2439, $\mathrm{m} / \mathrm{z}, \Delta=0.0004$ ), $[\mathrm{M}+3]^{+} \mathrm{m} / \mathrm{z}, 275.2463$ (calculated 275.2471, $\mathrm{m} / \mathrm{z} \Delta=-0.0008)$.

Haliclocyclin C (20): Colourless oil, $0.16 \mathrm{mg} ;{ }^{1} \mathrm{H}$ NMR $\left(\mathrm{CD}_{3} \mathrm{OD}, 600 \mathrm{MHz}\right) \delta 8.97$ (s, 1H, H-2), 8.86 (d, J=6.1 Hz, 1H, H-6), 8.50 (d, J=8.2 Hz, 1H, H-4), 8.06 (dd, $J=7.9,6.3 \mathrm{~Hz}, 1 \mathrm{H}, \mathrm{H}-5), 4.67$ (t, J=6.1 Hz, 2H, H-7), 2.96 (m, 2H, H-19), 2.07 (m, 2H, 
$\mathrm{H}-8), 1.84$ (m, 2H, H-18), 1.36-1.27 (m, 8H), 1.19-1.14 (m, 10H); ${ }^{13} \mathrm{C}$ NMR $\left(\mathrm{CD}_{3} \mathrm{OD}\right.$, $150 \mathrm{MHz}) \delta 147.2$ (C-4), 145.5 (C-2), 145.0 (C-3), 143.6 (C-6), 129.1 (C-5), 62.7 (C-7), 32.6, 31.3, 30.1, 28.1, 28.0, 27.94, 27.90, 27.50, 27.49, 27.4, 27.3, 25.2; HRESIMS $[\mathrm{M}]^{+} \mathrm{m} / \mathrm{z} 260.2381$ for $\mathrm{C}_{18} \mathrm{H}_{30} \mathrm{~N}^{+}$(calculated 260.2373, $\mathrm{m} / \mathrm{z} \Delta=0.0008$ ), $[\mathrm{M}+1]^{+}$ $\mathrm{m} / \mathrm{z} 261.2413$ (calculated 261.2406, $\mathrm{m} / \mathrm{z} \Delta=0.0007$ ), $[\mathrm{M}+2]^{+} \mathrm{m} / \mathrm{z} 262.2452$ (calculated $262.2439, m / z, \Delta=0.0013)$.

\subsubsection{Oxidation of Dehydrohaliclocyclin F}

In a biphasic solution of $\mathrm{CHCl}_{3}(2 \mathrm{~mL}), \mathrm{MeCN}(2 \mathrm{~mL})$ and $\mathrm{H}_{2} \mathrm{O}(3 \mathrm{~mL}), 100 \mu \mathrm{g}$ of dehydrohaliclocyclin $\mathrm{F}$ was dissolved. To this, $5 \mathrm{mg}$ of $\mathrm{NaIO}_{4}$ and $1 \mathrm{mg}$ of $\mathrm{RuCl}_{3}$ was added. The solution was stirred for two hours then filtered, washing with $\mathrm{CHCl}_{3}$ and $\mathrm{MeCN}$. The $\mathrm{CHCl}_{3}$ (VD4_49A) and $\mathrm{MeCN} / \mathrm{H}_{2} \mathrm{O}$ (VD4_49B) layers were separated and the solvent removed in vacuo. Samples were submitted for analysis by MS.

Dehydrohaliclocyclin F Oxidation Product (60): HRESIMS $[\mathrm{M}]^{+} \mathrm{m} / z, 304.2269$ for $\mathrm{C}_{19} \mathrm{H}_{30} \mathrm{NO}_{2}{ }^{+}$(calculated 304.2271, $m / z \Delta=-0.0002$ ); MS/MS fragments $m / z 192.1382$ for $\mathrm{C}_{12} \mathrm{H}_{18} \mathrm{NO}^{+}$(calculated 192.1383, $\mathrm{m} / z \Delta=-0.0001$ ), $\mathrm{m} / z .176 .1427$ for $\mathrm{C}_{12} \mathrm{H}_{18} \mathrm{~N}^{+}$ (calculated 176.1434, m/z $\Delta=-0.0007$ ), $m / z, 162.1268$ for $\mathrm{C}_{11} \mathrm{H}_{16} \mathrm{~N}^{+}$(calculated 162.1277, $\mathrm{m} / \mathrm{z} \Delta=-0.0009$ ), $\mathrm{m} / \mathrm{z} 120.0805$ for $\mathrm{C}_{8} \mathrm{H}_{10} \mathrm{~N}^{+}$(calculated 120.0808, $\mathrm{m} / \mathrm{z} \Delta$ $=-0.0003), m / z 106.0649$ for $\mathrm{C}_{7} \mathrm{H}_{8} \mathrm{~N}^{+}$(calculated 106.0651, $\mathrm{m} / \mathrm{z} \Delta=-0.0002$ ).

\subsubsection{VD4_47A}

A $104.5 \mathrm{mg}$ subsample of VD1_96B was further fractionated using a $30 \mathrm{~mL}$ column of cleaned HP20ss resin and eluted with $100 \mathrm{~mL}$ of $20 \%, 30 \%, 40 \%, 50 \%, 60 \%, 70 \%$, $80 \%$ and $100 \%$ methanol in water followed by $100 \%$ acetone. This produced fractions VD2_356A-N that were tested against $S$. cerevisiae (with the exception of the acetone wash VD2_356E) and ${ }^{1} \mathrm{H}$ NMR were run. Fraction VD2_356C had the highest mass (22.4 mg) and was amongst the most active fractions ( $\left.\mathrm{IC}_{50} 2.4 \pm 1.0 \mu \mathrm{g} / \mathrm{mL}\right)$. A full set of NMR experiments were run on this fraction to reveal a mixture of 3-APAs. Further purification of this extract was performed using reversed-phase (C18) semi-preparative HPLC, eluting with methanol in water (both containing $0.1 \%$ formic acid) with a flow rate of $4 \mathrm{~mL} / \mathrm{min}$. This was performed on the Agilent HPLC system. The compounds were eluted using a $62 \%$ methanol isocratic run for 20 minutes followed by a one minute ramp to $80 \%$ methanol, the solvent mixture was held at $80 \%$ methanol for five minutes before a one minute gradient brought the solvent mixture back down to $62 \%$ methanol where it was held for nine minutes (37 minutes total). The elution profile was followed using the ELSD trace and the fractions VD4_43A-J were collected. ${ }^{1} \mathrm{H}$ NMR spectra were 
collected for the fractions and 3-APAs were identified in almost all of them. Fractions VD4_43D (3.3 mg) and VD4_43E (3.8 mg), which were collected from two of three broad overlapping peaks, appeared to be the same compound in the ${ }^{1} \mathrm{H}$ NMR spectrum and so these were recombined and renamed to VD4_46A.

Samples VD4_43D and E were found to be poorly soluble when running NMR experiments using $\mathrm{CD}_{3} \mathrm{OD}$ so the counter-ion of the combined VD4_46A was exchanged using $\mathrm{NaCl}$. This was done by cyclic loading VD4_46A on to $2 \mathrm{~mL}$ of cleaned HP20ss resin. The sample was dissolved in $2 \mathrm{~mL}$ of methanol and run through the HP20ss column slowly, the eluent was collected and diluted with $2 \mathrm{~mL}$ of water to make a $50 \%$ methanol solution and this was run through the column again. This process was continued with eluent from the column being collected and diluted by $50 \%$ after each loading until a $3.125 \%$ methanol solution was run through the column. A saturated $\mathrm{NaCl}$ solution was then slowly passed through the column $(2 \times 15 \mathrm{~mL})$ and the column was washed with 5 $\times 30 \mathrm{~mL}$ of water. The column the finally eluted with $20 \mathrm{~mL}$ of methanol (VD4_47A) followed by $20 \mathrm{~mL}$ of acetone (VD4_47B). ${ }^{1} \mathrm{H}$ NMR spectra showed that VD4_47A, with a mass of $4.4 \mathrm{mg}$, contained the 3-APA. A full set of NMR experiments was therefore run on this fraction, however the structure was not able to be elucidated in this study, see Section 4.4.4.

\subsection{Extraction of MNP_1001}

Frozen Callyspongia sp. (MNP_1001, 112 g) was extracted twice in methanol (200 mL). These extracts were then filtered through a pad of celite to remove any solid material. The methanol extracts were cyclic loaded onto $45 \mathrm{~mL}$ of cleaned HP20 resin with the second extract being run through the column followed by the first extract. The eluent from these extractions was collected together and $400 \mathrm{~mL}$ of water was added to this to make a $50 \%$ methanol solution. This solution was then passed through the column again and $800 \mathrm{~mL}$ of water was added to the eluent to make a $25 \%$ methanol solution. This $25 \%$ methanol solution was then passed through the column. The column, which was now loaded with the MNP_1001 extract, was then eluted using $135 \mathrm{~mL}$ of $0 \%, 20 \%, 40 \%, 60 \%, 80 \%$ and $100 \%$ acetone in water.

The $20 \%, 40 \%, 60 \%$ and $80 \%$ acetone in water fractions were each backloaded on to $15 \mathrm{~mL}$ of cleaned HP20 resin in order to remove the water. To do this, the fractions were diluted with a further $135 \mathrm{~mL}$ of water and run through the column slowly to load the extract fraction onto the column. The column was then eluted with $45 \mathrm{~mL}$ of methanol followed by $45 \mathrm{~mL}$ of acetone, these elutions were collected together and the solvent removed in vacuo. The $100 \%$ acetone fraction was also dried in vacuo. The $20 \%, 40 \%$, $60 \%, 80 \%$ and $100 \%$ fractions were labelled VD1_91A, B, C, D and E, respectively. 
These fractions were transferred to pre-weighed sample vials using methanol (VD1_91A, $\mathrm{B}$ and $\mathrm{C}$ ) or $20 \% \mathrm{CH}_{2} \mathrm{Cl}_{2}$ in methanol (VD1_91D and $\mathrm{E}$ ) and dried in vacuo. To recover masses of $4.9 \mathrm{mg}$ (VD1_91A), $9.0 \mathrm{mg}$ (VD1_91B), $10.0 \mathrm{mg}$ (VD1_91C), $32.9 \mathrm{mg}$ (VD1_91D) and 72.3 mg (VD1_91E).

These fractions were tested against $S$. cerevisiae in a dose-response assay twice, testing maximum concentrations of $200 \mu \mathrm{g} / \mathrm{mL}$ and $100 \mu \mathrm{g} / \mathrm{mL}$. These assays both showed that the fractions had no activity against $S$. cerevisiae, including for a recombined fraction tested in the second assay. The lack of activity and low masses recovered of from this sponge extraction resulted in this work being discontinued.

\subsection{Isolation of Compounds from PTN3_40G}

The ascidian PTN3_40G (17.92 g) was completely extracted in methanol (100 mL) and the small amount of remaining solid was then reextracted $(25 \mathrm{~mL})$. These extracts were then filtered to remove the minor solid material remaining. The methanol extracts were cyclic loaded onto $25 \mathrm{~mL}$ of cleaned HP20 resin, with the second extract being run through the column followed by the first. The eluent from these extractions was collected together and $125 \mathrm{~mL}$ of water was added to this to make a $50 \%$ methanol solution. This solution was then passed through the column again and $250 \mathrm{~mL}$ of water was added to the eluent to make a $25 \%$ methanol solution. This $25 \%$ methanol solution was then passed through the column. The column, which was now loaded with the PTN3_40G extract, was then eluted using $75 \mathrm{~mL}$ of $30 \%, 75 \%$ and $100 \%$ acetone in water.

The $30 \%$ and $75 \%$ acetone in water fractions were each backloaded on to $10 \mathrm{~mL}$ of cleaned HP20 resin in order to remove the water. In order to do this, the fractions were diluted with a further $75 \mathrm{~mL}$ of water and run through the column slowly, the eluent was then diluted with another $80-100 \mathrm{~mL}$ of water and run through the column again to load the extract fraction onto the column. The column was then eluted with $50 \mathrm{~mL}$ of methanol followed by $50 \mathrm{~mL}$ of acetone, these elutions were collected together and the solvent removed in vacuo. The $100 \%$ acetone fraction was also dried in vacuo. The $30 \%$, $75 \%$ and $100 \%$ fractions were labelled VD3_64A, B and C, respectively. These fractions were transferred to pre-weighed sample vials using $20 \% \mathrm{CH}_{2} \mathrm{Cl}_{2}$ in methanol and dried in vacuo. To recover masses of $9.5 \mathrm{mg}$ (VD3_64A), $51.1 \mathrm{mg}$ (VD3_64B) and $213.9 \mathrm{mg}$ (VD3_64C). These fractions were tested against $S$. cerevisiae in a dose-response assay, testing a maximum concentration of $100 \mu \mathrm{g} / \mathrm{mL}$. This assay showed that the fractions had poor activity against $S$. cerevisiae with $27 \%$ of yeast growth inhibited at a concentration of $100 \mu \mathrm{g} / \mathrm{mL}$ for VD4_64A and B and no inhibition observed for VD4_64C.

Fraction VD3_64B was further fractionated using $11 \mathrm{~mL}$ of cleaned HP20ss resin and 
eluted with $33 \mathrm{~mL}$ of $20 \%, 40 \%, 60 \%, 80 \%$ and $100 \%$ methanol in water and $100 \%$ acetone. This produced samples VD3_80A-H that were tested against $S$. cerevisiae and ${ }^{1} \mathrm{H}$ NMR were run. Poor to no inhibition was found in these fractions again so an NMR-guided approach was used for further fractionation. Fraction VD3_80C that had been isolated from the acetone strip of the HP20ss column had a mass of $14.1 \mathrm{mg}$ and showed some interesting resonances in the ${ }^{1} \mathrm{H}$ NMR spectrum so it was selected for further purification. Further purification of VD3_80C was done using a $12 \mathrm{~mL}$ Diol column, eluting with $40 \mathrm{~mL}$ of $100 \%, 50 \%$ and $0 \%$ hexanes in ethyl acetate followed by $25 \%, 50 \%, 75 \%$ and $100 \%$ methanol in ethyl acetate, finally the column was stripped with $25 \%$ and $50 \%$ water in methanol. This produced fractions VD3_98A-O, the ${ }^{1} \mathrm{H}$ NMR of these fractions enabled the fatty acid VD3_98G and the phthalate ester VD3_98E to be selected for full characterisation. A full set of NMR experiments were run on the fatty acid VD3_98G, strengthening the proposal that the structure was a fatty acid.

Before a full set of NMR experiments could be run on the VD3_98E, it needed to be further purified using normal-phase (Diol) analytical HPLC purification, eluting with ethyl acetate in hexanes. This was performed on the Rainin HPLC system. A 15 minute isocratic elution of 7\% ethyl acetate in hexanes was used with a flow rate of $1 \mathrm{~mL} / \mathrm{min}$ in this fractionation. The elution profile was followed using the UV/Vis trace at 210 and $270 \mathrm{~nm}$, the fractions VD4_17A, B and C were collected. The strong major peak in the UV/Vis trace was collected as VD4_17B, this was thought to be the phthalate ester. A full set of NMR experiments was run on VD4_17B (3.2 mg) revealing that this fraction was a mixture of phthalate ester structures.

Fatty Acid VD398G tentatively assigned as cis-vaccenic acid (13): White solid, $1.3 \mathrm{mg} ;{ }^{1} \mathrm{H} \mathrm{NMR}\left(\mathrm{CDCl}_{3}, 600 \mathrm{MHz}\right) \delta 5.35(\mathrm{t}, J=4.5,2 \mathrm{H}), 2.22(\mathrm{t}, J=7.7 \mathrm{~Hz}, 2 \mathrm{H}), 2.01$ (d, $J=6.6 \mathrm{~Hz}, 4 \mathrm{H}$ ), 1.63 (quin, $J=7.4 \mathrm{~Hz}, 2 \mathrm{H}$ ), 1.35-1.24 (m, 22H), 0.88 (t, $J=7.1 \mathrm{~Hz}, 3 \mathrm{H}$ ); ${ }^{13} \mathrm{C} \mathrm{NMR}\left(\mathrm{CDCl}_{3}, 150 \mathrm{MHz}\right) \delta 175.6,130.1,130.0,36.1,32.1,29.9,29.8,29.75,29.7$, 29.68, 29.6, 29.49, 29.48, 29.4, 27.4, 25.7, 22.9, 14.3 .

Phthalate Ester Mixture VD4_17B: Colourless oil, $3.2 \mathrm{mg} ;{ }^{1} \mathrm{H}$ NMR $\left(\mathrm{CD}_{3} \mathrm{OD}\right.$, $600 \mathrm{MHz}) \delta 7.72(\mathrm{~m}, 2 \mathrm{H}), 7.62(\mathrm{~m}, 2 \mathrm{H}), 4.36-4.26(\mathrm{~m}), 1.81-1.08$ (indistinguishable multiplets), $1.06-0.79$ (indistinguishable multiplets); the ${ }^{13} \mathrm{C} \mathrm{NMR}\left(\mathrm{CD}_{3} \mathrm{OD}, 150 \mathrm{MHz}\right)$ had many broad and overlapping resonances due to the mixture of compounds and so is not reported (see Appendix D.3).

\subsection{Isolation of Steroids from PTN2_67C}

Frozen Leucosolenia sp. (PTN2_67C, $93 \mathrm{~g}$ ) was extracted twice in methanol (250 mL). These extracts were then filtered through a pad of celite to remove any solid material. 
The methanol extracts were cyclic loaded onto $40 \mathrm{~mL}$ of cleaned HP20 resin, with the second extract being run through the column followed by the first. The eluent from these extractions was collected together and $500 \mathrm{~mL}$ of water was added to this to make a $50 \%$ methanol solution. This solution was then passed through the column again and $1 \mathrm{~L}$ of water was added to the eluent to make a $25 \%$ methanol solution. This $25 \%$ methanol solution was then passed through the column. The column, which was now loaded with the PTN2_67C extract, was then eluted using $120 \mathrm{~mL}$ of $30 \%, 75 \%$ and $100 \%$ acetone in water.

The $30 \%$ and $75 \%$ acetone in water fractions were each backloaded on to $15 \mathrm{~mL}$ of cleaned HP20 resin in order to remove the water. To do this, the fractions were diluted with a further $120 \mathrm{~mL}$ of water and run through the column slowly, the eluent was then diluted with another $240 \mathrm{~mL}$ of water and run through the column again to load the extract fraction onto the column. The column was then eluted with $60 \mathrm{~mL}$ of methanol followed by $60 \mathrm{~mL}$ of acetone, these elutions were collected together and the solvent removed in vacuo. The $100 \%$ acetone fraction was also dried in vacuo. The $30 \%, 75 \%$ and $100 \%$ fractions were labelled VD3_68A, B and C, respectively. These fractions were transferred to pre-weighed sample vials using $20 \% \mathrm{CH}_{2} \mathrm{Cl}_{2}$ in methanol and dried under a stream of compressed air to recover masses of $30.5 \mathrm{mg}$ (VD3_68A), $185.5 \mathrm{mg}$ (VD3_68B) and $209.7 \mathrm{mg}$ (VD3_68C). These fractions were tested against $S$. cerevisiae in a dose-response assay testing a maximum concentration of $100 \mu \mathrm{g} / \mathrm{mL}$. This assay gave approximate $\mathrm{IC}_{50}$ values of $16 \pm 1 \mu \mathrm{g} / \mathrm{mL}$ for VD3_68A and $25 \pm 1 \mu \mathrm{g} / \mathrm{mL}$ for VD3_68B and showed no inhibition for VD2_68C. ${ }^{1} \mathrm{H}$ NMR spectra were also collected for all of these fractions. Fraction VD3_68B was chosen for further purification because of its activity and high mass. Fraction VD3_68A was not pursued further because of its relatively low mass.

A $92.8 \mathrm{mg}$ subsample of VD3_68B was further fractionated using a $12 \mathrm{~mL}$ column of cleaned HP20ss resin and eluted with $36 \mathrm{~mL}$ of $20 \%, 40 \%, 60 \%, 80 \%$ and $100 \%$ methanol in water followed by $100 \%$ acetone. This produced fractions VD3_79A-F that were tested against $S$. cerevisiae and ${ }^{1} \mathrm{H}$ NMR were run. Fraction VD3_79B had the highest mass (30.1 mg) and was the most active fraction $\left(\mathrm{IC}_{50} 94 \pm 2 \mu \mathrm{g} / \mathrm{mL}\right)$. Further purification of VD3_79B was done using a $13 \mathrm{~mL}$ Diol column, eluting with $40 \mathrm{~mL}$ of $0 \%, 25 \%$, $50 \%, 75 \%$ and $100 \%$ ethyl acetate in hexanes followed by $25 \%, 50 \%, 75 \%$ and $100 \%$ methanol in ethyl acetate, finally the column was stripped with $25 \%$ and $50 \%$ water in methanol. This produced fractions VD4_05A-Y, the ${ }^{1} \mathrm{H}$ NMR of these showed that fractions VD4_05K, L and M all contained a steroid structure so these fractions were combined and a full set of NMR experiments were run on this combined fraction. The NMR data showed a mixture of trihydroxylated steroids were present in the combined VD4_05KLM fraction so further purification was required.

Further purification of VD4_05KLM was performed using reversed-phase (C18) analytical HPLC, eluting with methanol (containing $0.1 \%$ formic acid) in water and using a flow 
rate of $1 \mathrm{~mL} / \mathrm{min}$. This was performed on the Agilent HPLC system. The compounds were eluted using a 15 minute $90 \%$ methanol isocratic run followed by a one minute ramp to $100 \%$ methanol, the solvent mixture was held at $100 \%$ methanol for five minutes before a one minute ramp brought the solvent mixture back down to $90 \%$ methanol where it was held for eight minutes (30 minutes total). The elution profile was followed using the ELSD trace and the fractions VD5_71A-M were collected. ${ }^{1} \mathrm{H}$ NMR spectra were collected for the fractions. VD5_71J was selected to run a full set of NMR experiments on, in order to solve the structure, because it was a clean fraction with the largest mass $(0.3 \mathrm{mg})$. The structure of VD5_71J (14) was proposed based on the NMR data and this was found to be a known compound.

Trihydroxylated steroid VD5_71J: Colourless oil, $0.3 \mathrm{mg} ;{ }^{1} \mathrm{H} \mathrm{NMR}\left(\mathrm{CD}_{3} \mathrm{OD}, 600 \mathrm{MHz}\right)$ $\delta 5.27(\mathrm{~m}, 1 \mathrm{H}, \mathrm{H}-7), 5.21-5.19(\mathrm{~m}, 2 \mathrm{H}, \mathrm{H}-22 \& \mathrm{H}-23), 3.97$ (tt, J=11.4, $4.8 \mathrm{~Hz}, 1 \mathrm{H}$, H-3), 3.54 (m, 1H, H-6), 2.12-2.08 (m, 1H, H-4a), 2.04 (s, 1H, H-20), 2.01 (s, 1H, H-9), 1.98-1.94 (m, 1H, H-14), 1.84 (q, J=6.6 Hz, 1H, H-24), 1.81-1.74 (m, 2H, H-2a), 1.69 (ddd, $J=13.3,4.8,2.0,1 \mathrm{H}, \mathrm{H}-4 \mathrm{~b}$ ), 1.63-1.54 (m, 4H, H-1a, H-11a \& H-11b), 1.53-1.44 (m, $3 \mathrm{H}, \mathrm{H}-1 \mathrm{~b}, \mathrm{H}-2 \mathrm{~b} \& \mathrm{H}-25$ ) 1.32 (m, 1H, H-17), 1.06 (s, 3H, H-19), 1.03 (d, J=6.6 Hz, 3H, H-21), 0.94 (d, J=6.8 Hz, 3H, H-28), 0.87 (d, J=6.8 Hz, 3H, H-26), 0.85 (d, $J=6.8 \mathrm{~Hz}, 3 \mathrm{H}$, $\mathrm{H}-27), 0.65$ (s, 3H, H-18); ${ }^{13} \mathrm{C} \mathrm{NMR}^{*}\left(\mathrm{CD}_{3} \mathrm{OD}, 600 \mathrm{MHz}\right) \delta 137.0$ (C-22), 133.0 (C-23), 118.8 (C-7), 68.1 (C-3), 73.9 (C-6), 57.0 (C-17), 55.6 (C-14), 44.3 (C-24), 44.0 (C-9), 41.7 (C-20), 34.2 (C-25), 76.6 (C-5), 44.4 (C-13), 37.8 (C-10), 40.1 (C-4), 33.6 (C-1), 31.5 (C-2), 40.4 (C-12), 21.4 (C-21), 20.4 (C-26), 19.8 (C-27), 18.6 (C-19), 18.4 (C-28), 12.4 (C-18); HRESIMS [M+Na] ${ }^{+} m / z 453.3305$ for $\mathrm{C}_{28} \mathrm{H}_{46} \mathrm{O}_{3} \mathrm{Na}^{+}$(calculated 453.3345, $m / z \Delta=-0.0040)$.

*obtained from HSQC and HMBC spectra 


\section{Appendix A}

\section{NMR Spectra of Dehydrohaliclocyclin C}

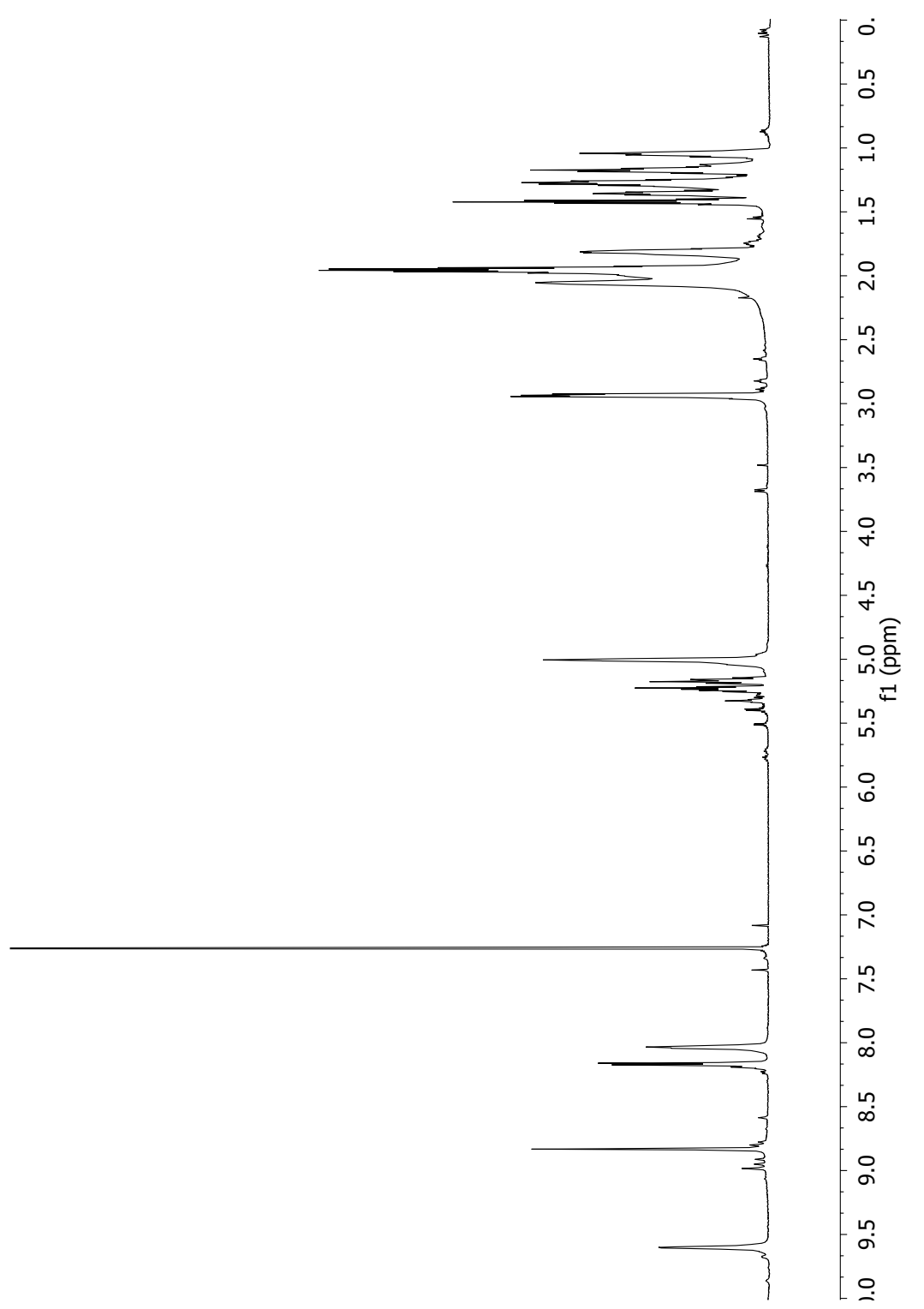

${ }^{1} \mathrm{H}$ NMR spectrum of dehydrohaliclocyclin $\mathrm{C}(\mathbf{5 8})\left(600 \mathrm{MHz}, \mathrm{CDCl}_{3}\right)$ 


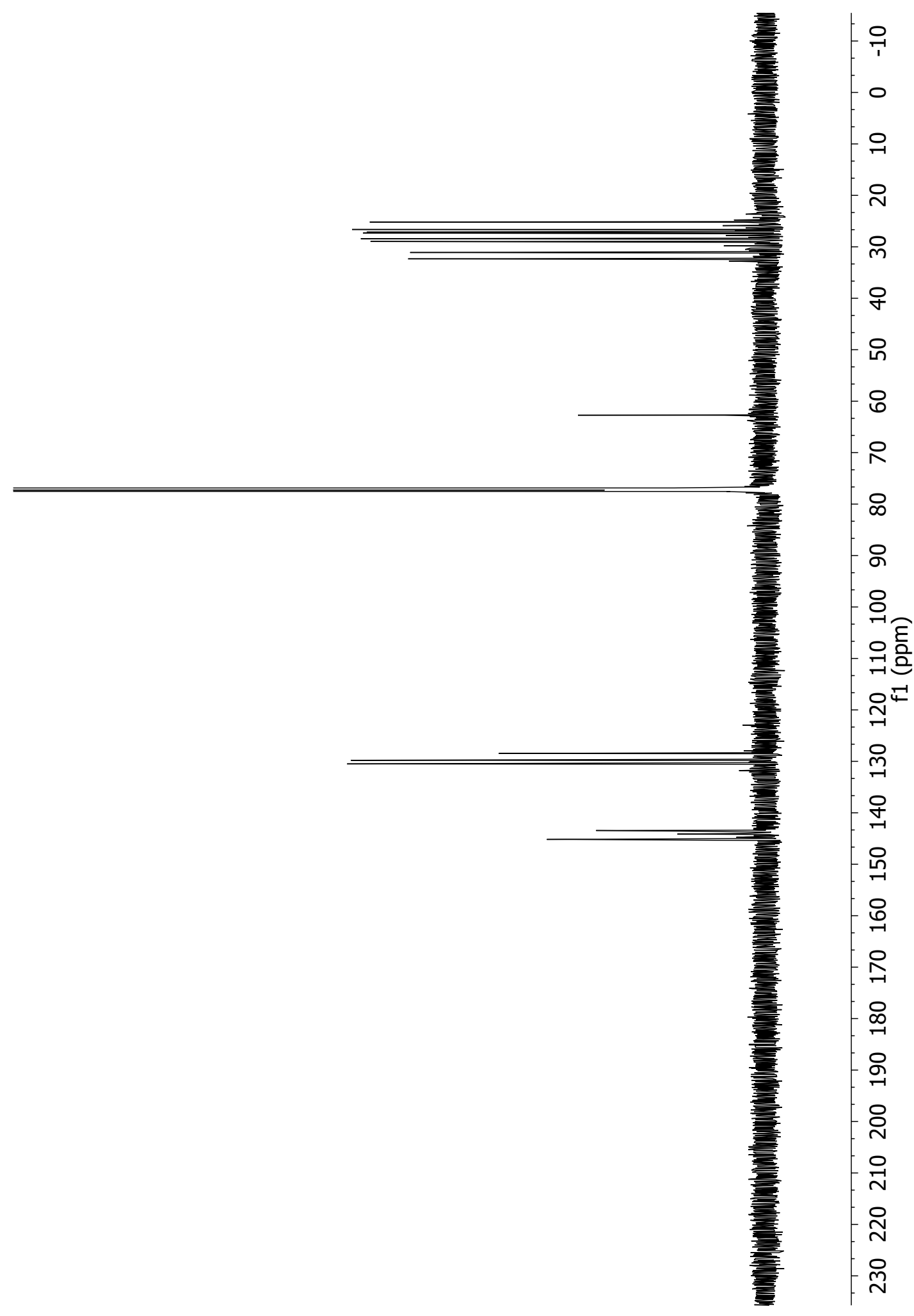

${ }^{13} \mathrm{C}$ NMR spectrum of dehydrohaliclocyclin $\mathrm{C}(\mathbf{5 8})\left(150 \mathrm{MHz}, \mathrm{CDCl}_{3}\right)$ 
(udd) If

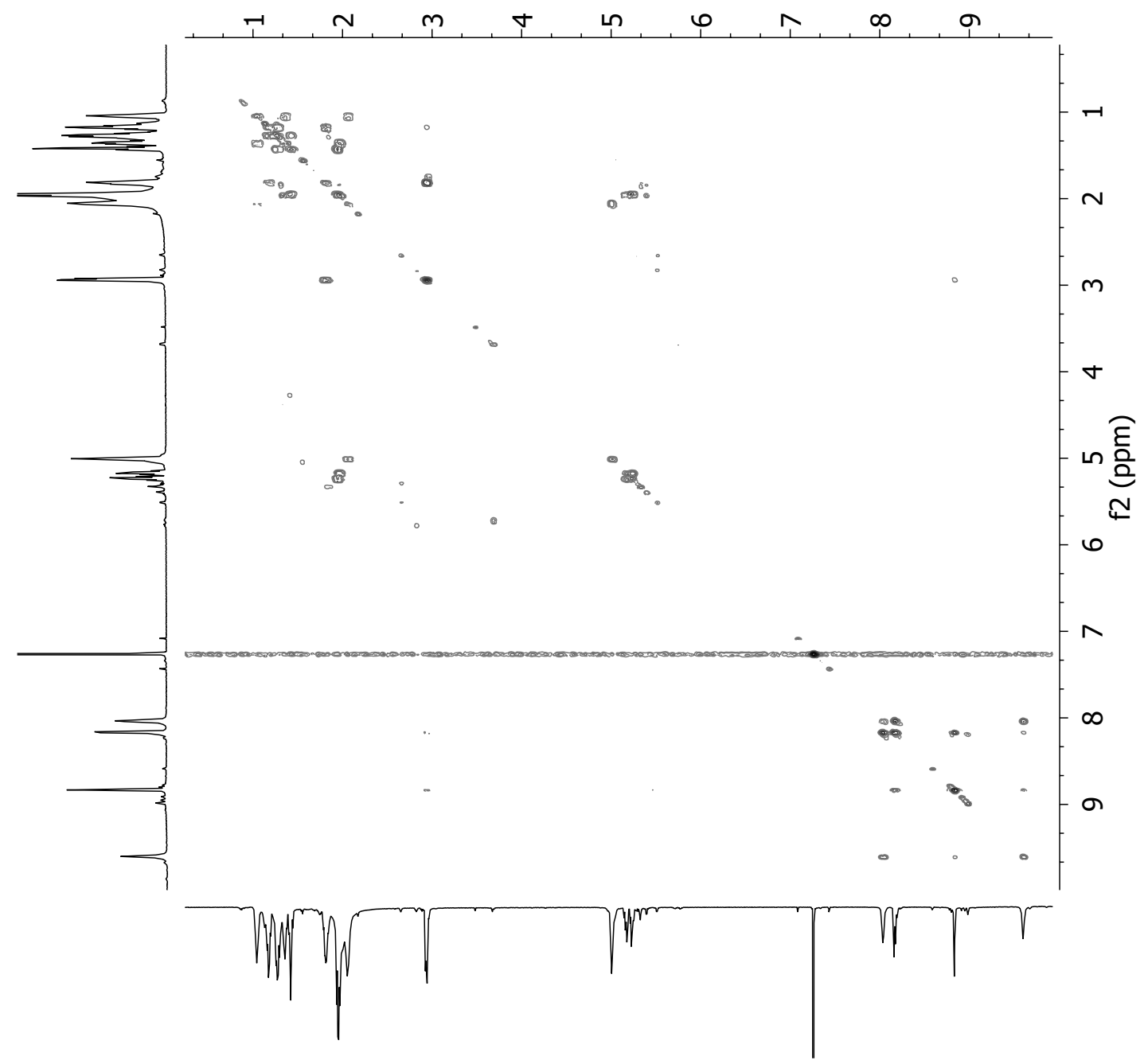

COSY spectrum of dehydrohaliclocyclin C (58) $\left(600 \mathrm{MHz}, \mathrm{CDCl}_{3}\right)$ 


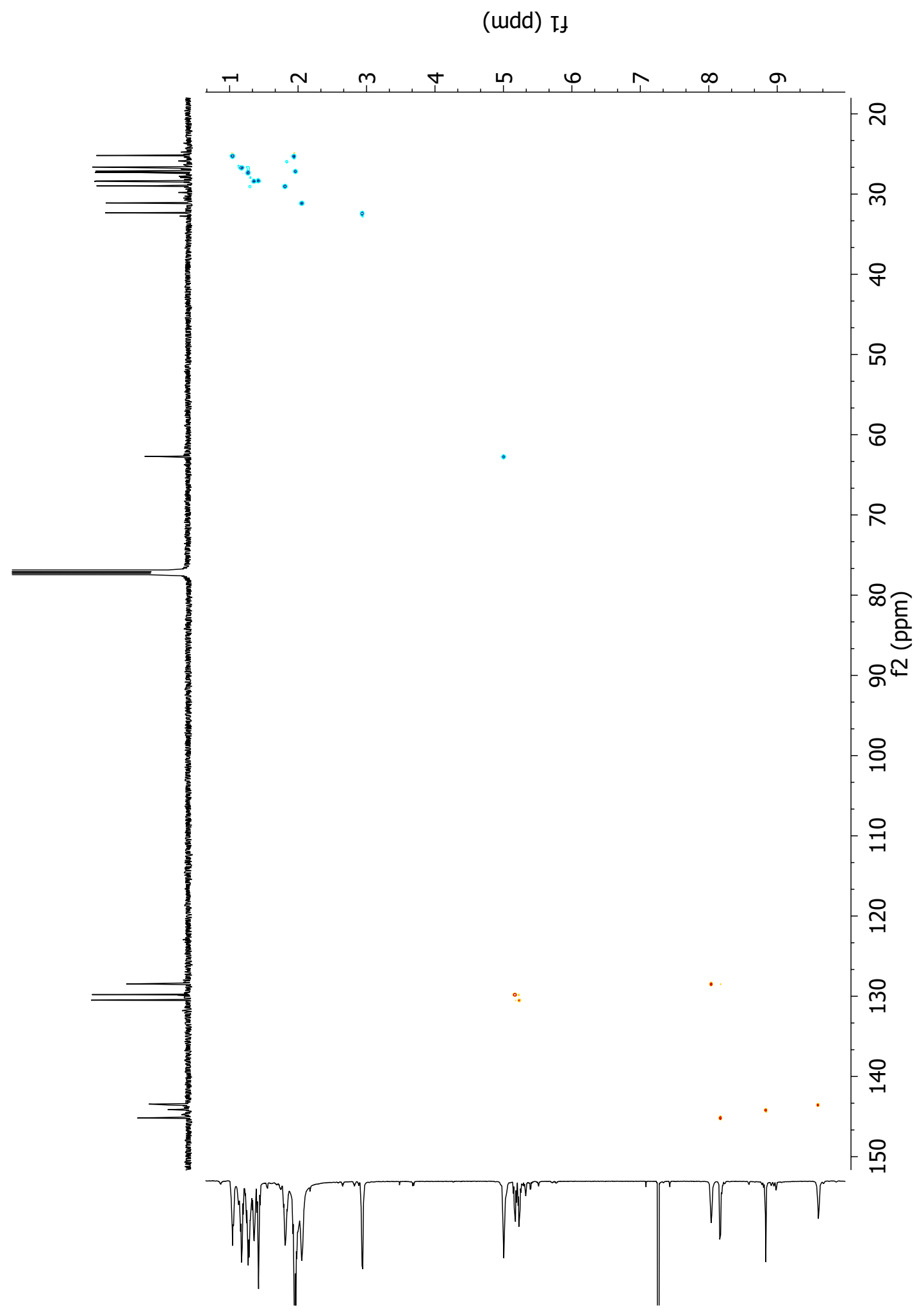

Mutliplicity-edited HSQC spectrum of dehydrohaliclocyclin C (58) showing methylenes in blue and methyls and methines in red $\left(600 \mathrm{MHz}, \mathrm{CDCl}_{3}\right)$ 


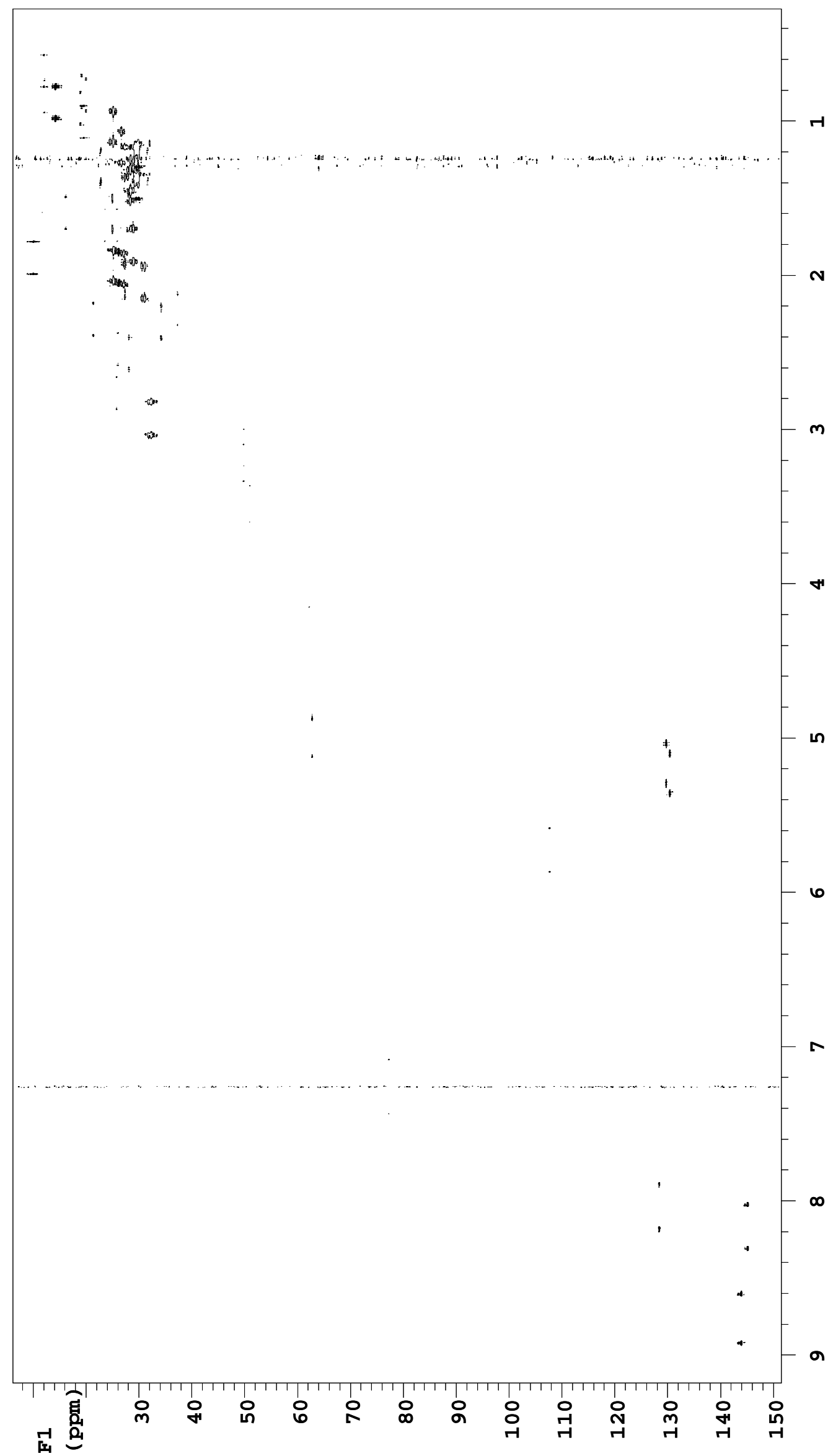

Fully coupled HSQC spectrum of dehydrohaliclocyclin C (58) $\left(600 \mathrm{MHz}, \mathrm{CDCl}_{3}\right)$ 


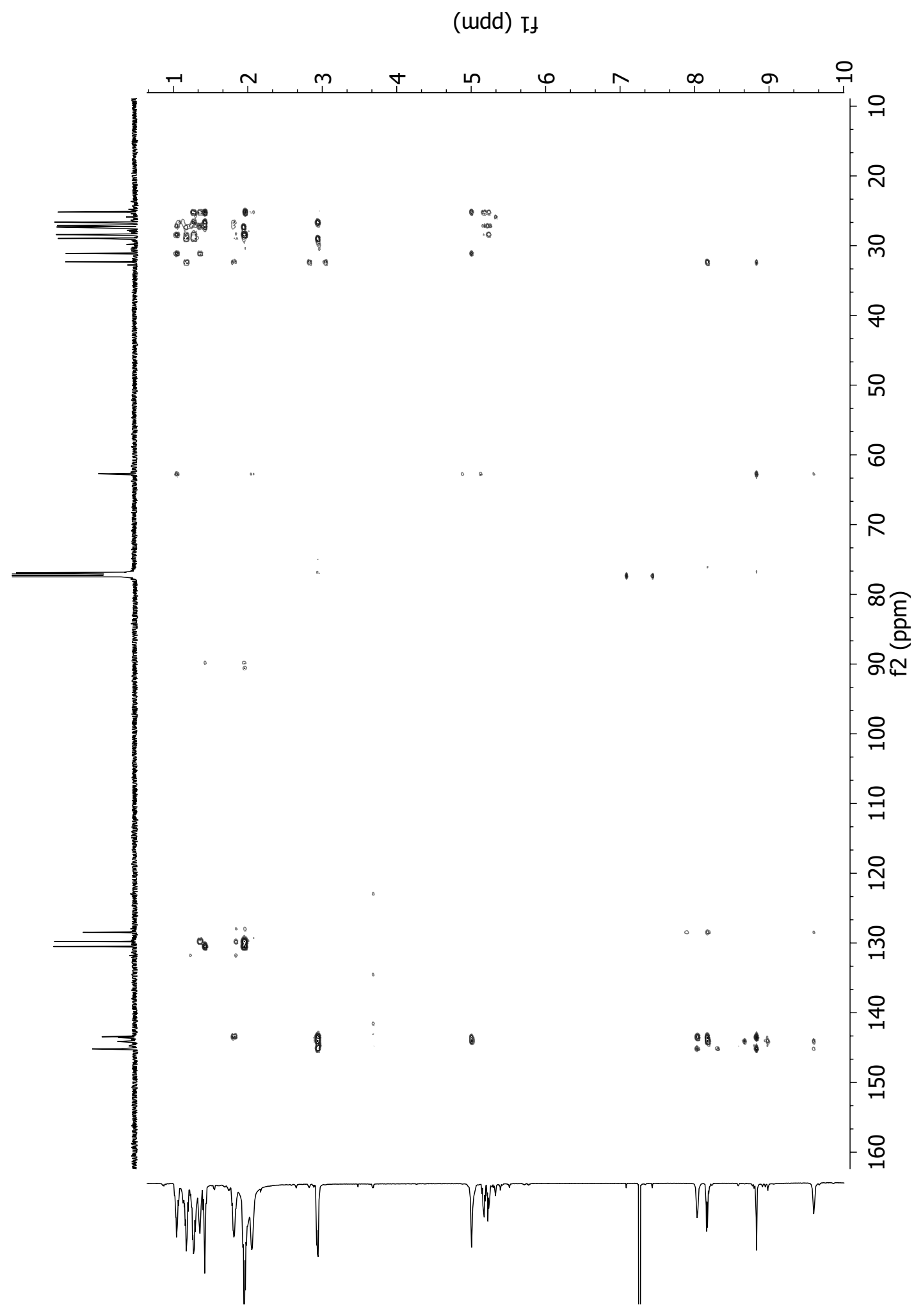

HMBC spectrum of dehydrohaliclocyclin C (58) $\left(600 \mathrm{MHz}, \mathrm{CDCl}_{3}\right)$ 


\section{Appendix B}

\section{NMR Spectra of Dehydrohaliclocyclin F}

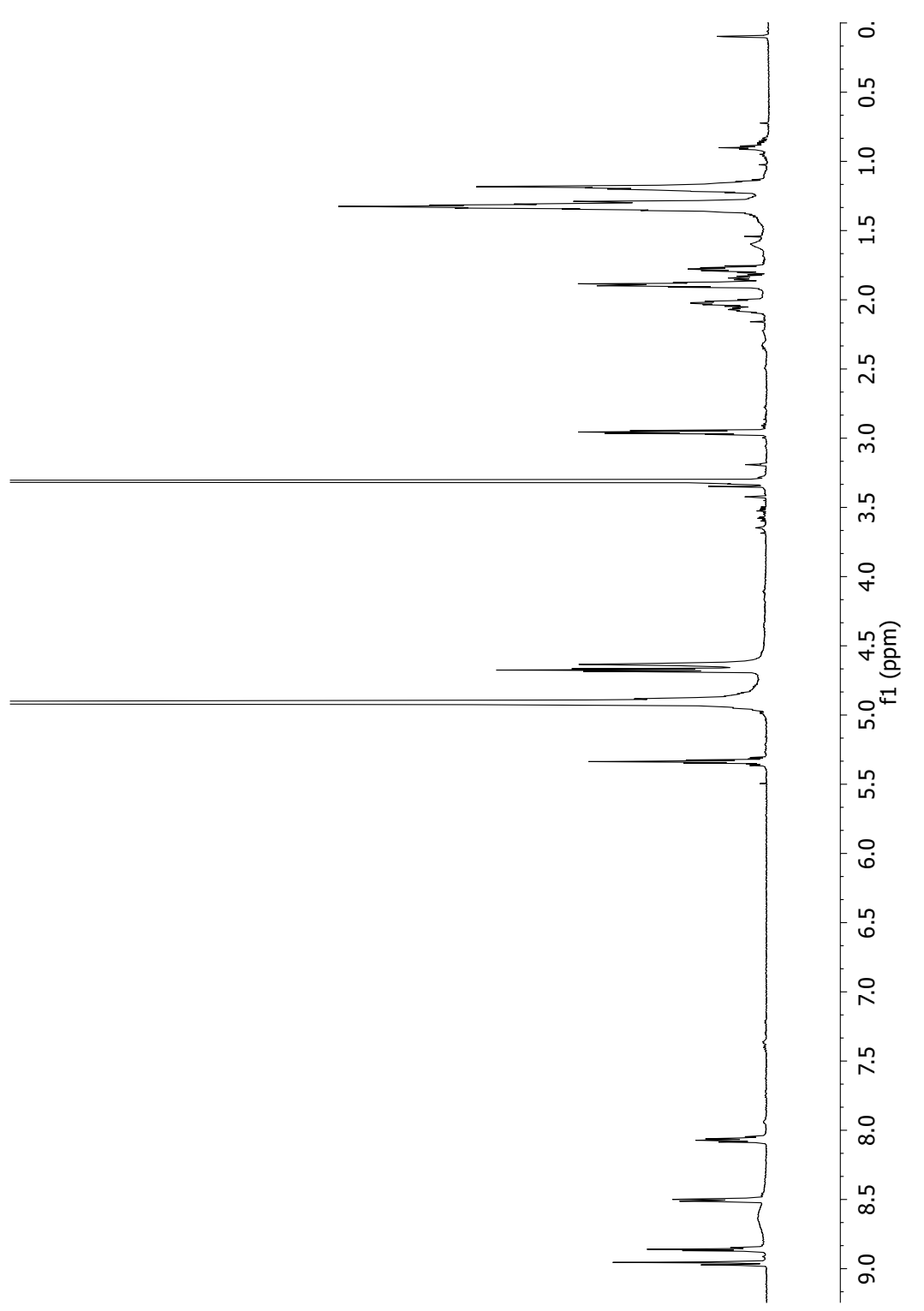

${ }^{1} \mathrm{H}$ NMR spectrum of dehydrohaliclocyclin F (59) (600 MHz, $\left.\mathrm{CD}_{3} \mathrm{OD}\right)$ 


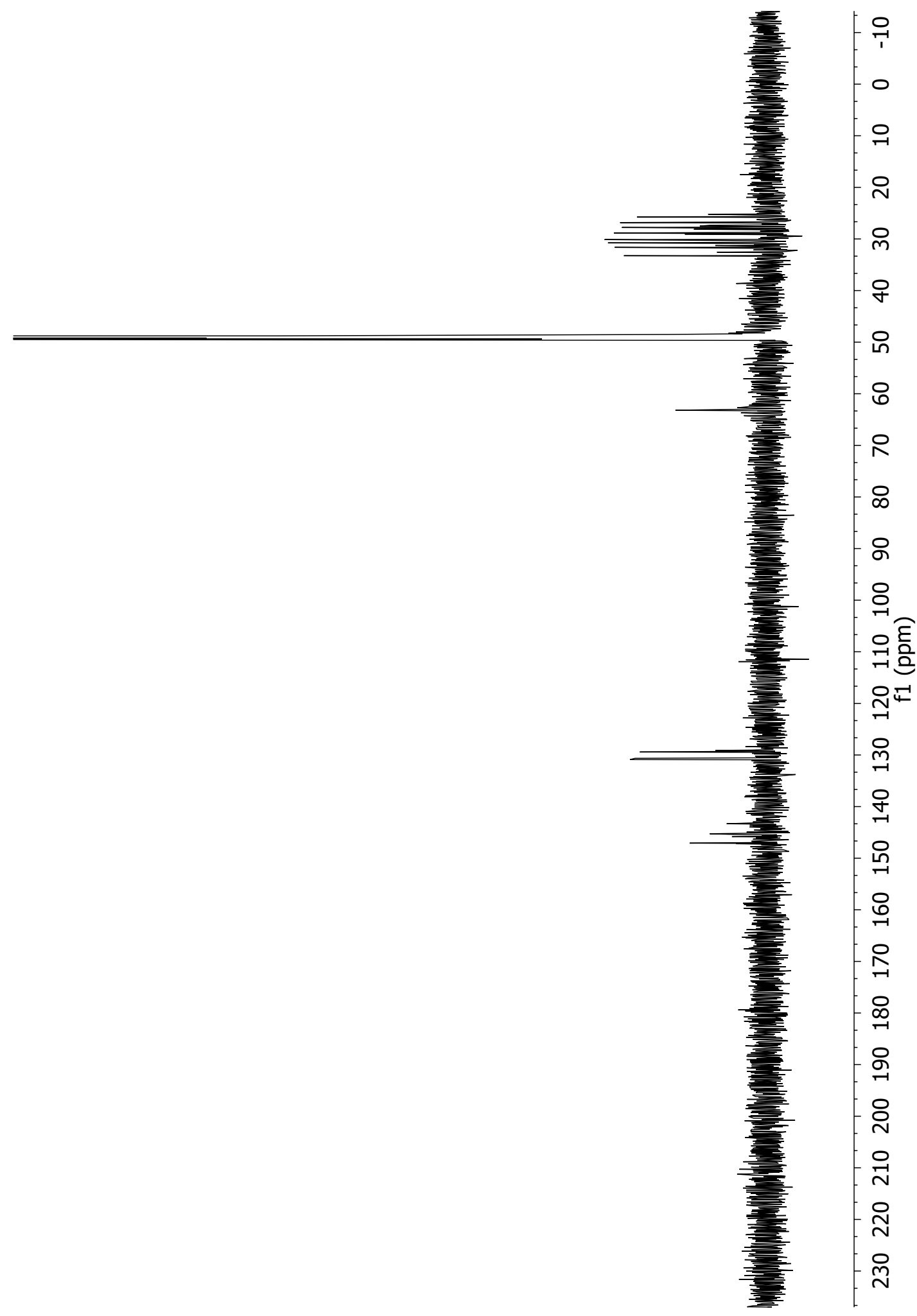

${ }^{13} \mathrm{C}$ NMR spectrum of dehydrohaliclocyclin F (59) (150 MHz, $\left.\mathrm{CD}_{3} \mathrm{OD}\right)$ 
(udd) If

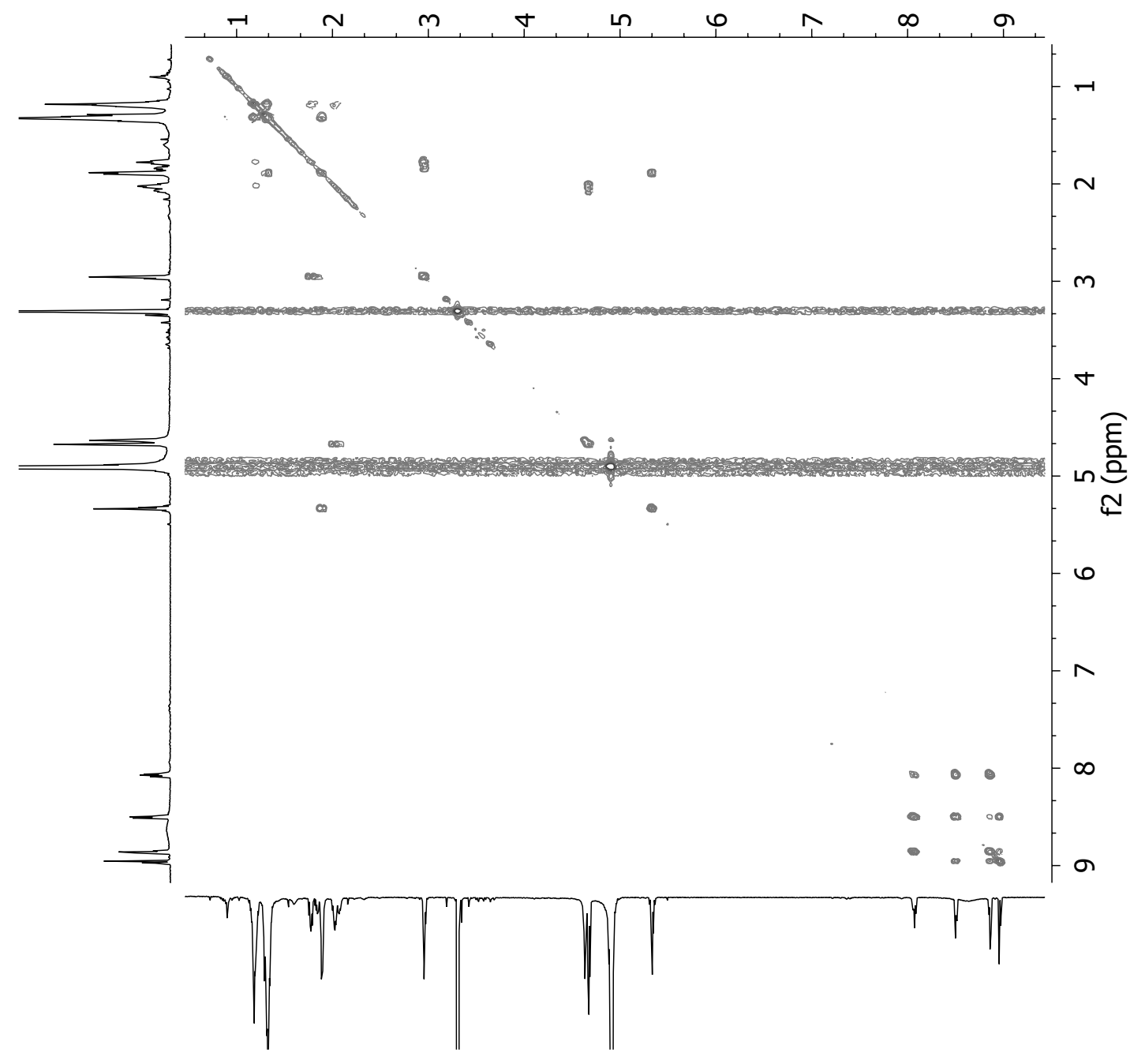

COSY spectrum of dehydrohaliclocyclin F (59) (600 MHz, $\left.\mathrm{CD}_{3} \mathrm{OD}\right)$ 


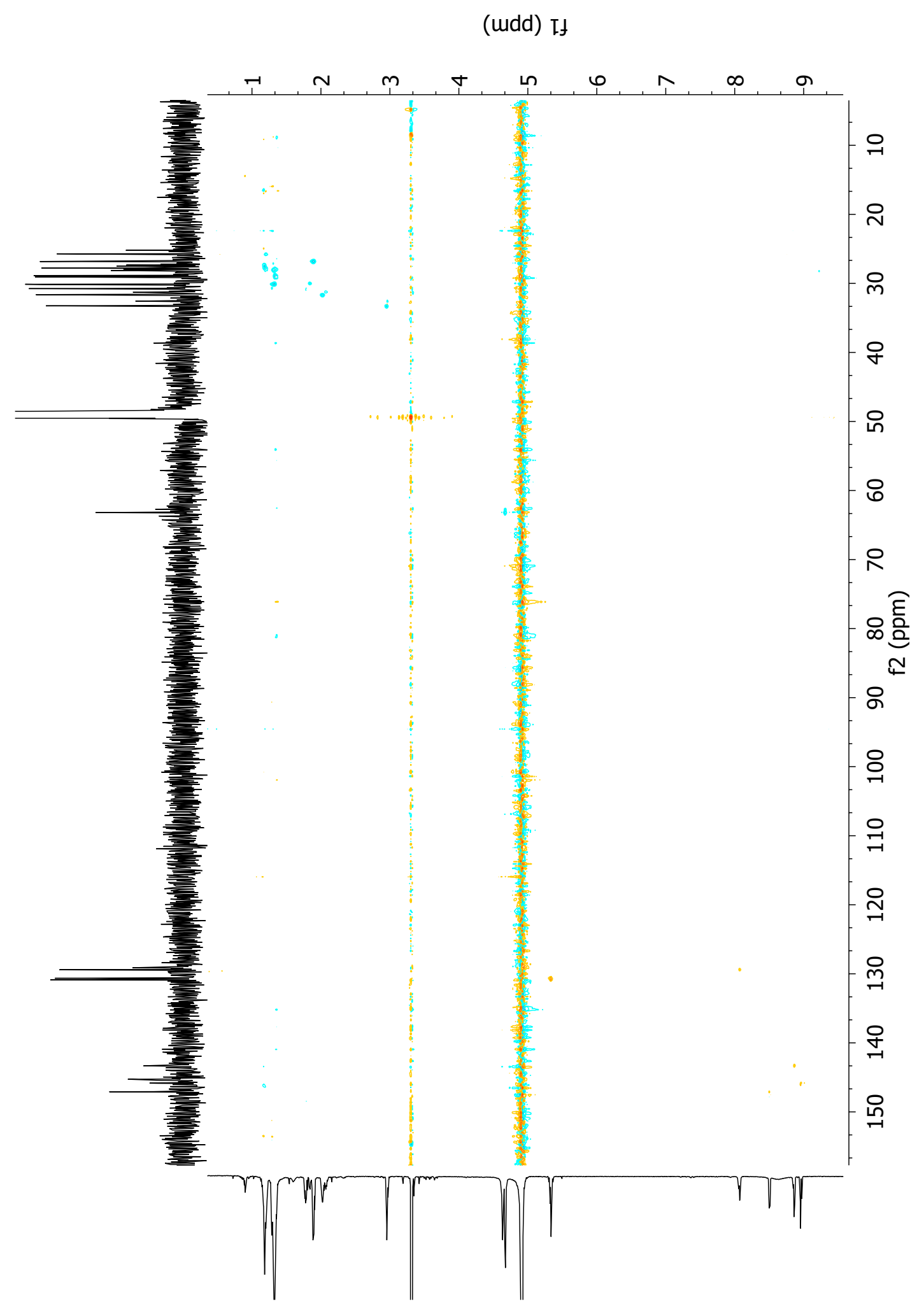

Mutliplicity-edited HSQC spectrum of dehydrohaliclocyclin F (59) showing methylenes in blue and methyls and methines in red $\left(600 \mathrm{MHz}, \mathrm{CD}_{3} \mathrm{OD}\right)$ 


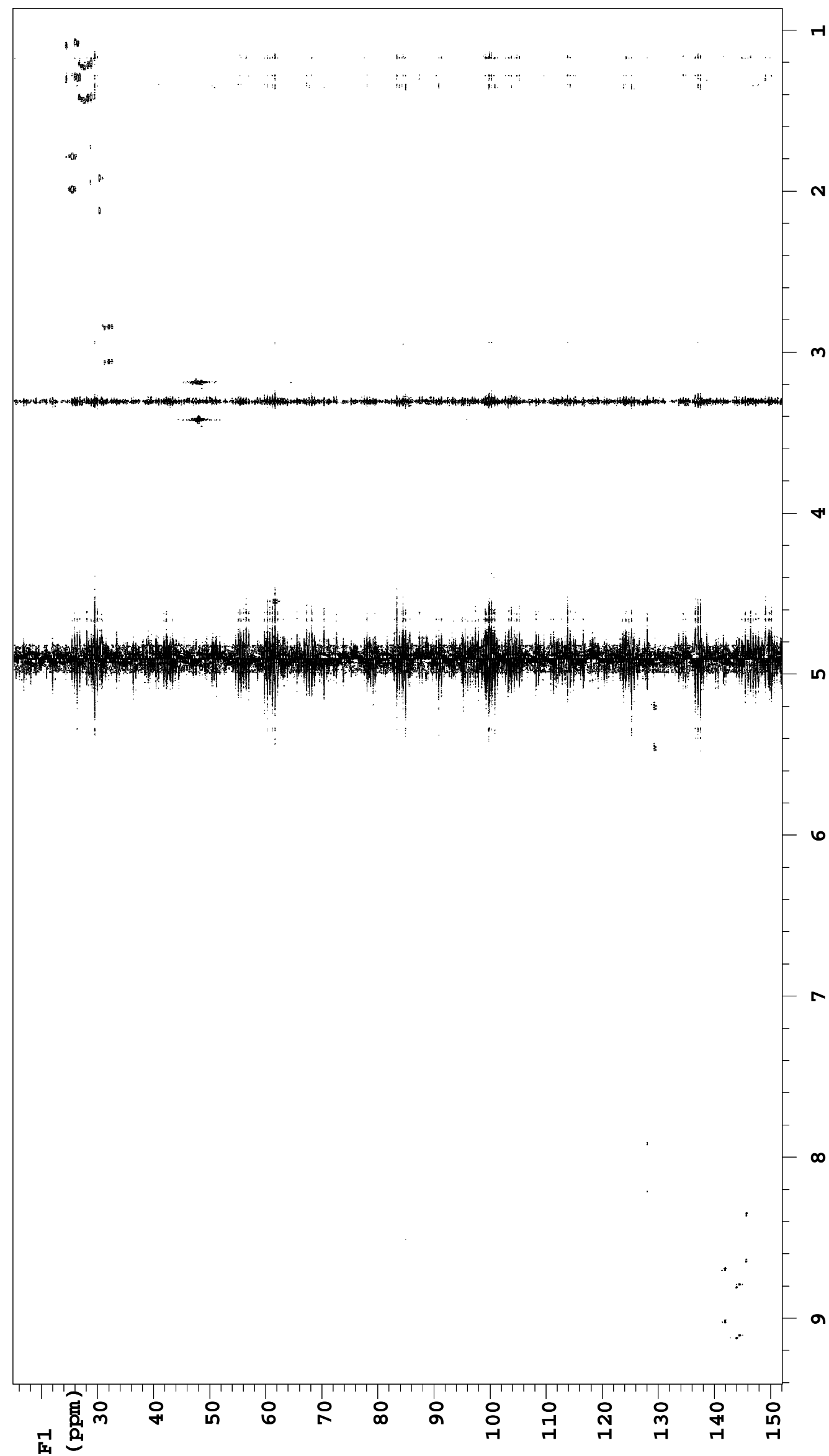

Fully coupled HSQC spectrum of dehydrohaliclocyclin F (59) (600 MHz, $\left.\mathrm{CD}_{3} \mathrm{OD}\right)$ 


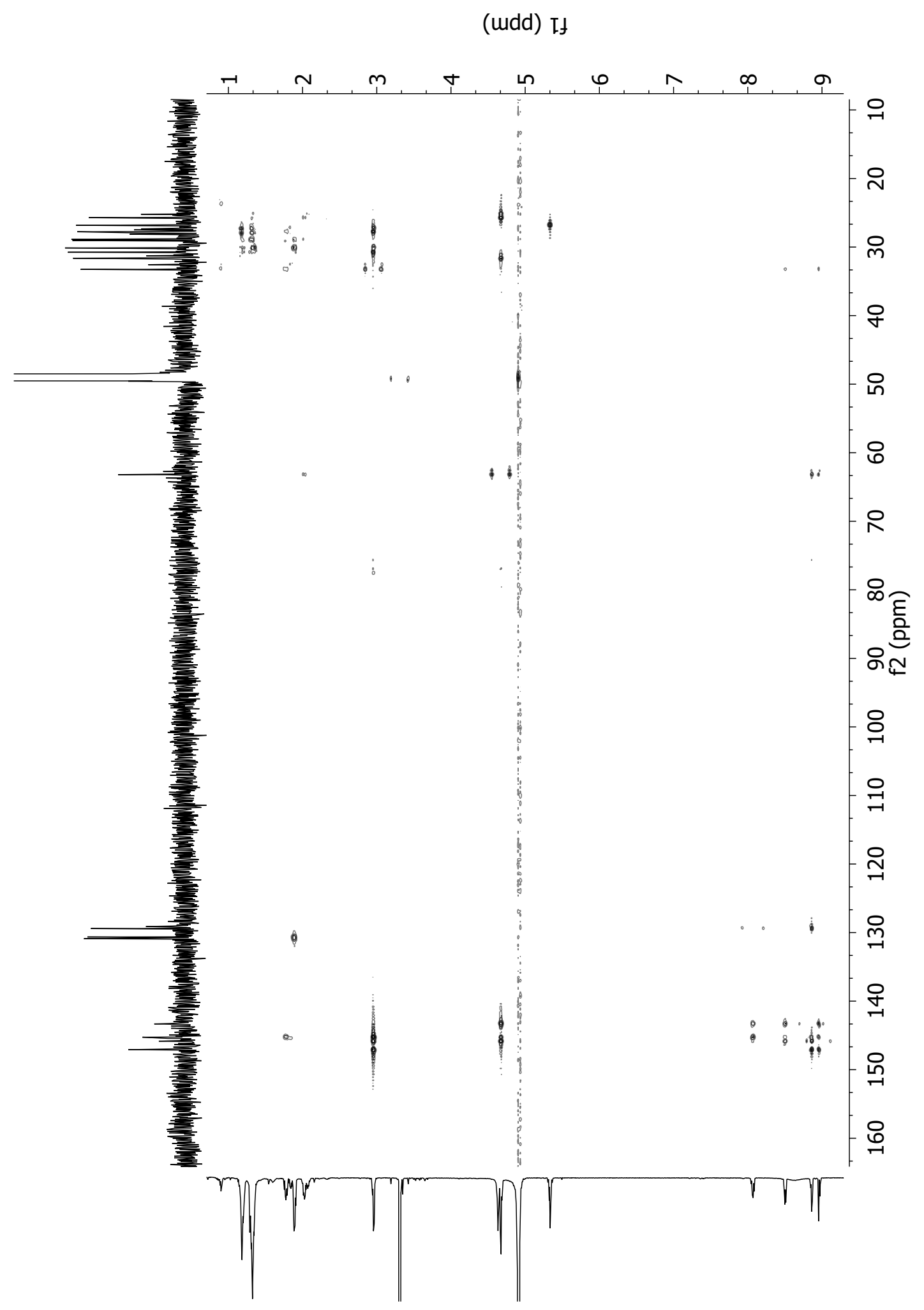

HMBC spectrum of dehydrohaliclocyclin F (59) (600 MHz, $\left.\mathrm{CD}_{3} \mathrm{OD}\right)$ 


\section{Appendix C}

\section{NMR Spectra of Known Compounds}

\section{C.1 Haliclocyclin C}

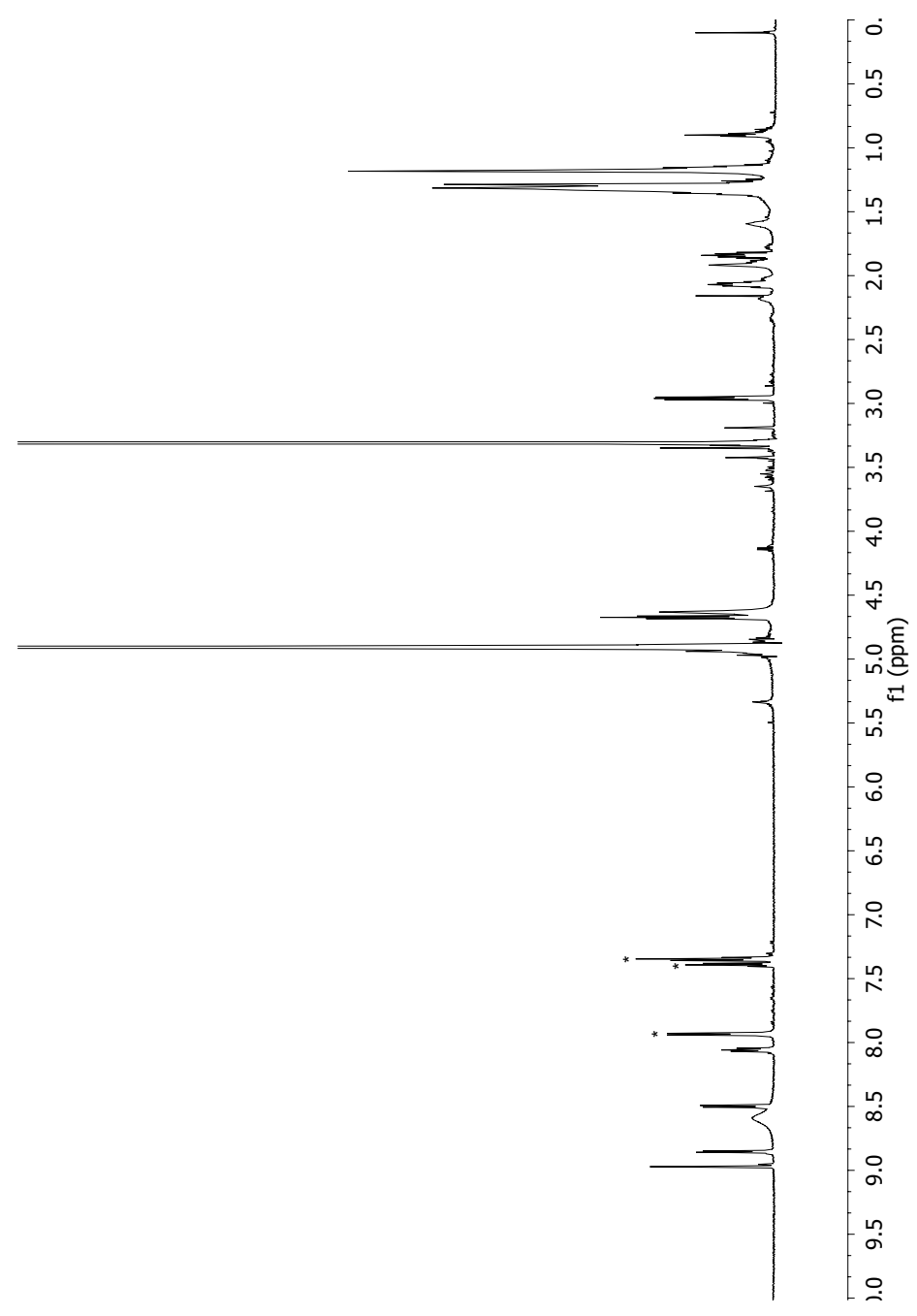

${ }^{1} \mathrm{H}$ NMR spectrum of haliclocyclin C (20) $\left(600 \mathrm{MHz}, \mathrm{CD}_{3} \mathrm{OD}\right)$

$*$ = contamination 


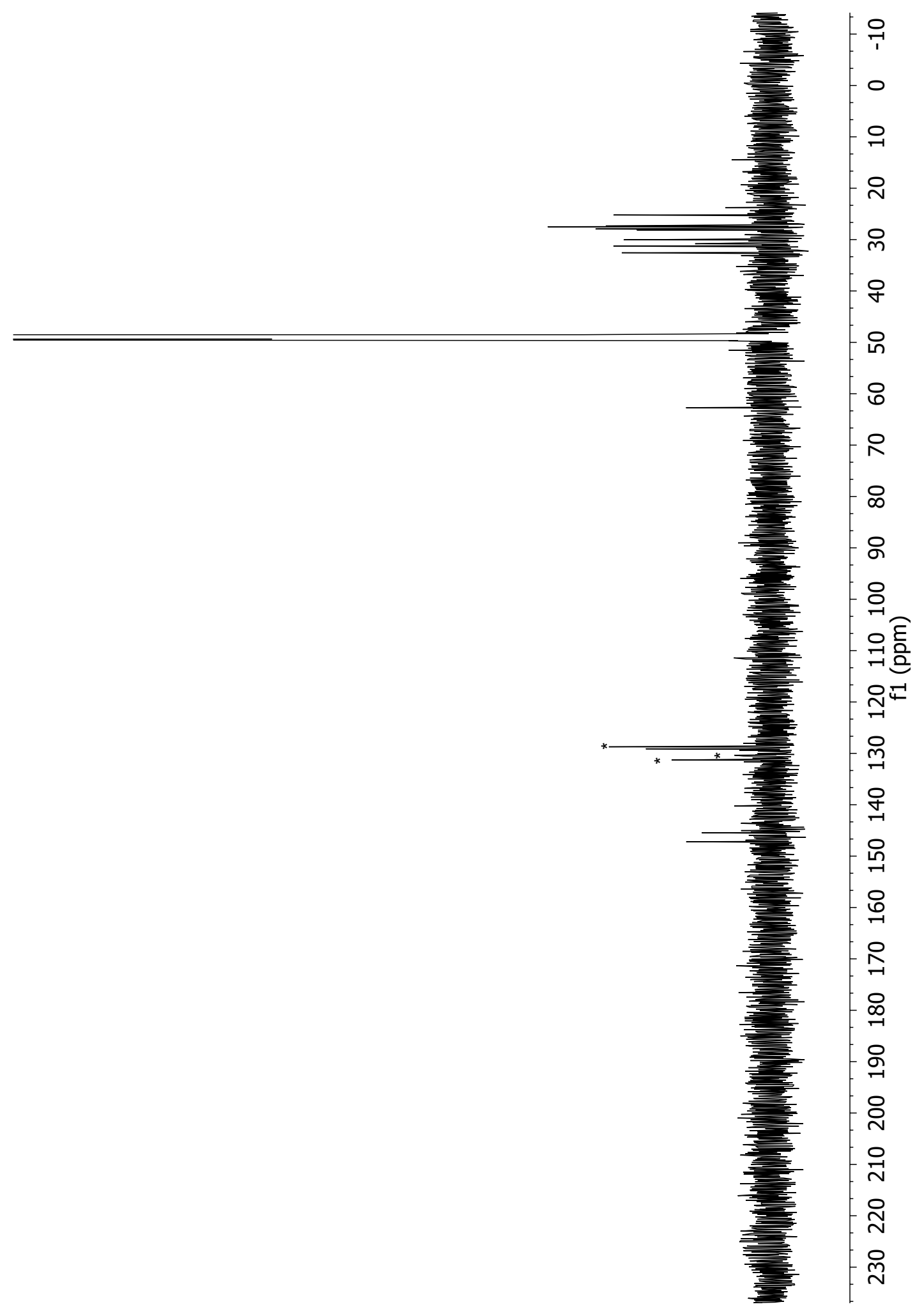

${ }^{13} \mathrm{C}$ NMR spectrum of haliclocyclin $\mathrm{C}(\mathbf{2 0})\left(150 \mathrm{MHz}, \mathrm{CD}_{3} \mathrm{OD}\right)$

$*=$ contamination 


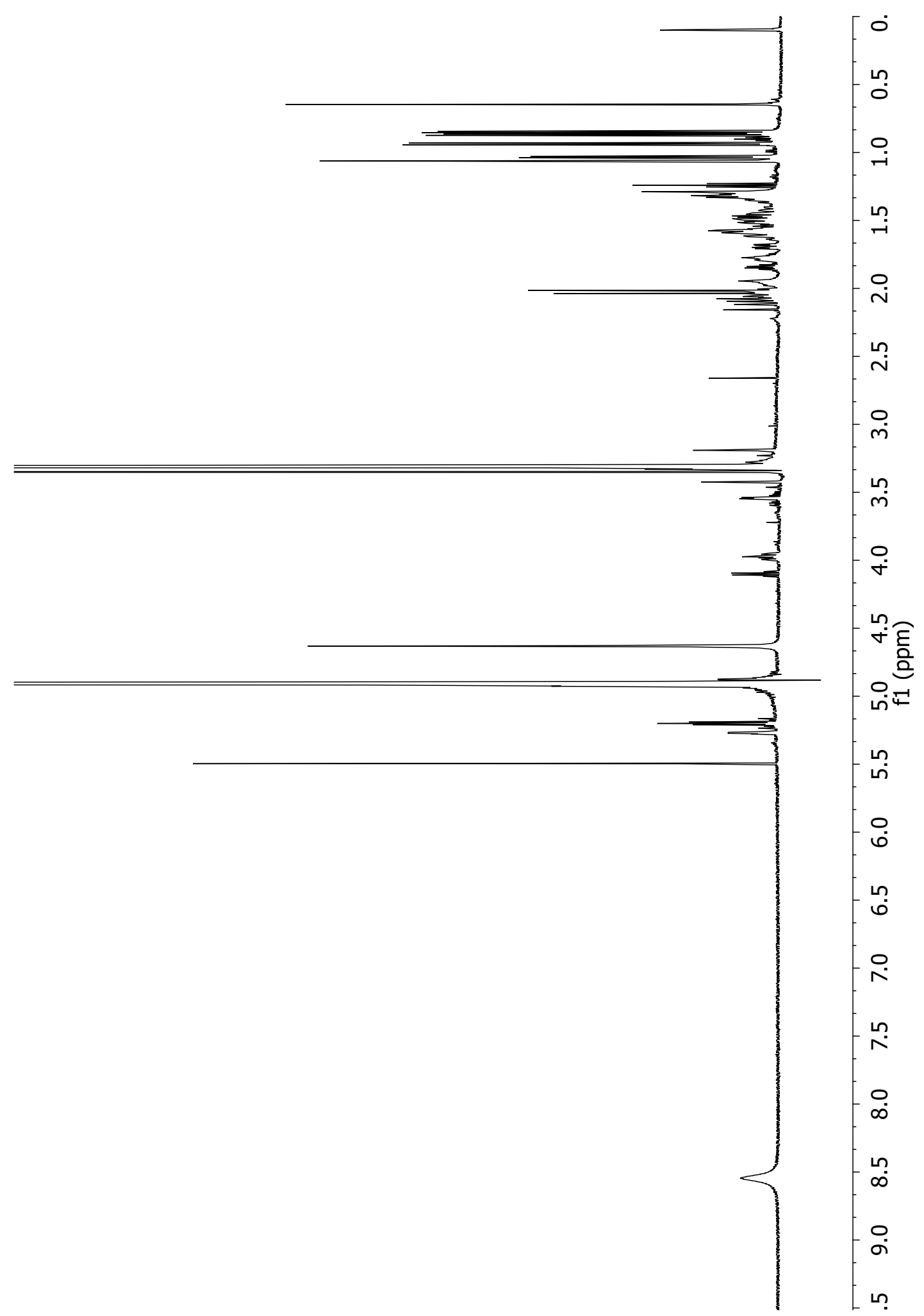

${ }^{1} \mathrm{H}$ NMR spectrum of the steroid VD5_17J (600 MHz, $\left.\mathrm{CD}_{3} \mathrm{OD}\right)$ 


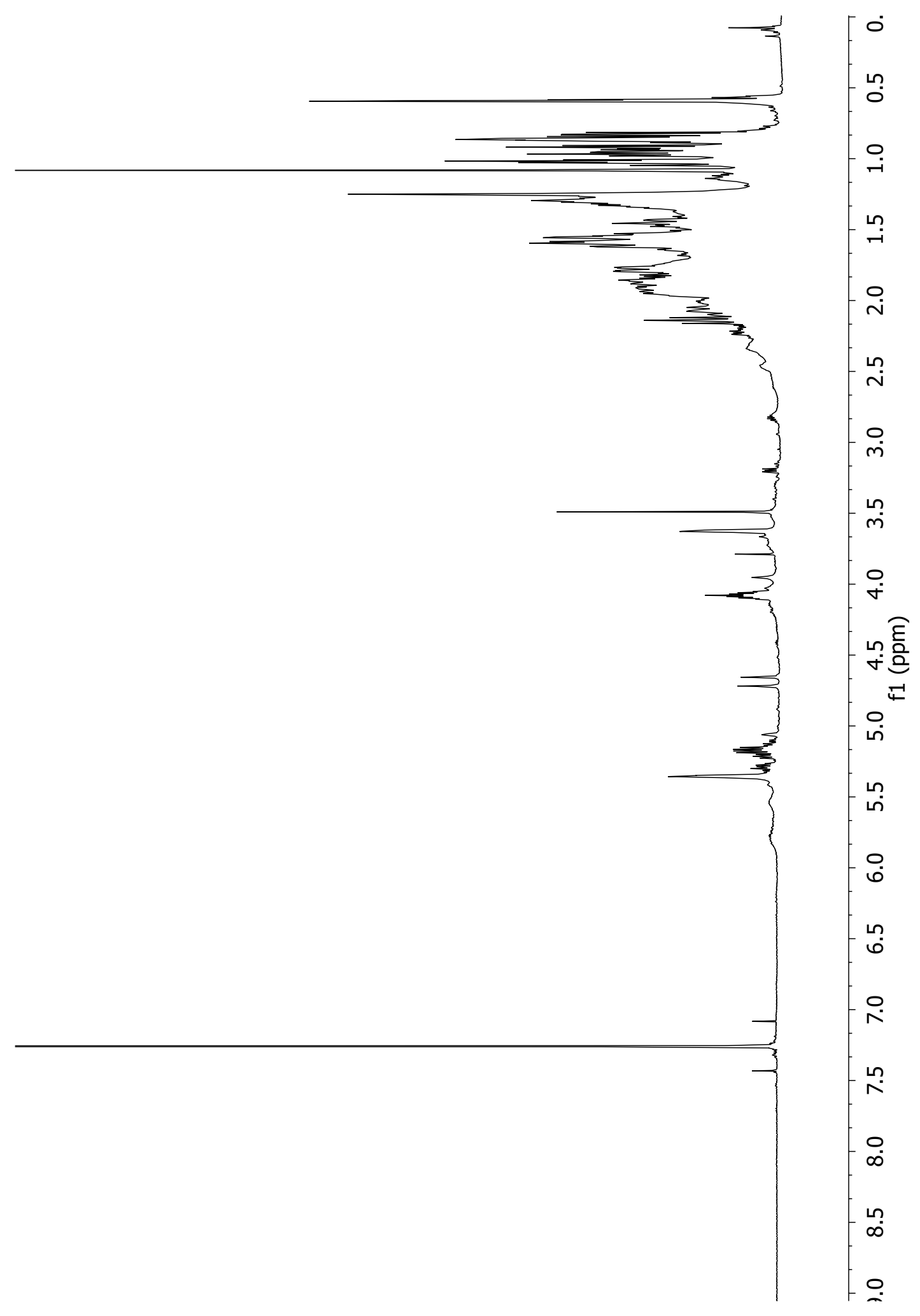

${ }^{1} \mathrm{H}$ NMR spectrum of the steroid mixture VD4_05KLM (600 MHz, $\mathrm{CDCl}_{3}$ ) 


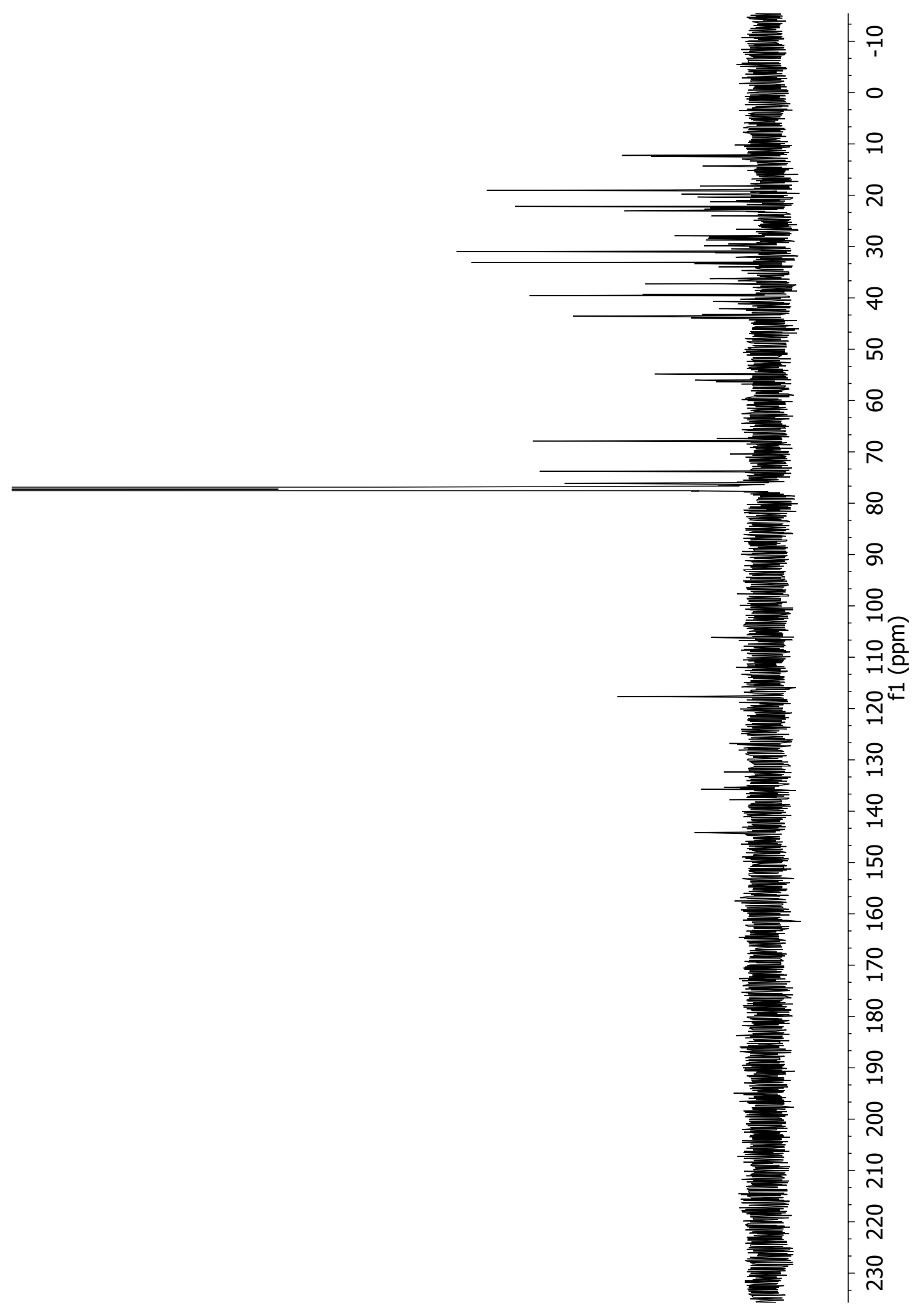

${ }^{13} \mathrm{C}$ NMR spectrum of steroid mixture VD4_05KLM (150 MHz, $\mathrm{CDCl}_{3}$ ) 


\title{
Appendix D
}

\section{NMR Spectra of Discontinued Projects}

\author{
D.1 3-APA VD4_47A
}

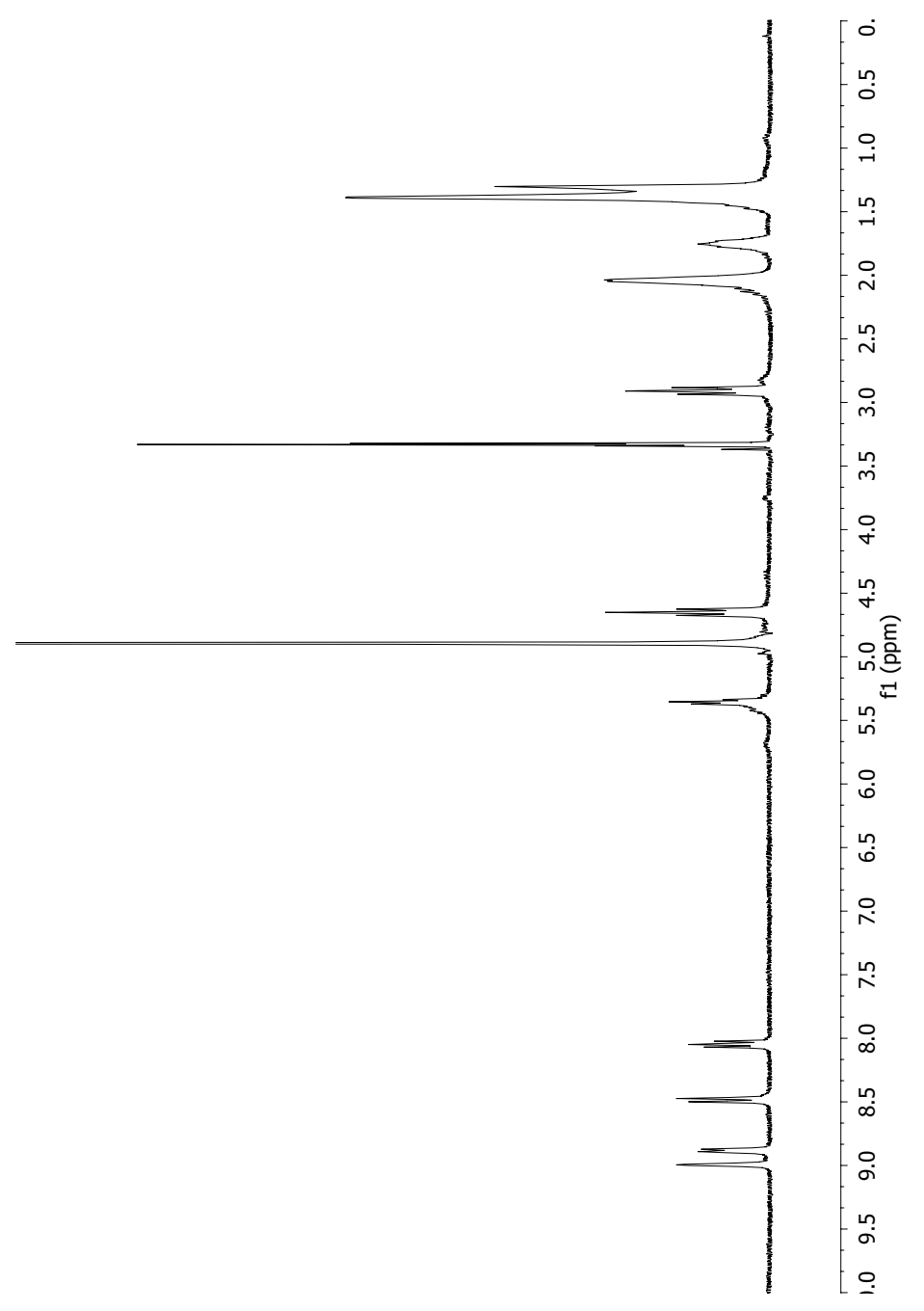

${ }^{1} \mathrm{H}$ NMR spectrum of the 3-APA VD4_47A (600 MHz, $\left.\mathrm{CD}_{3} \mathrm{OD}\right)$ 


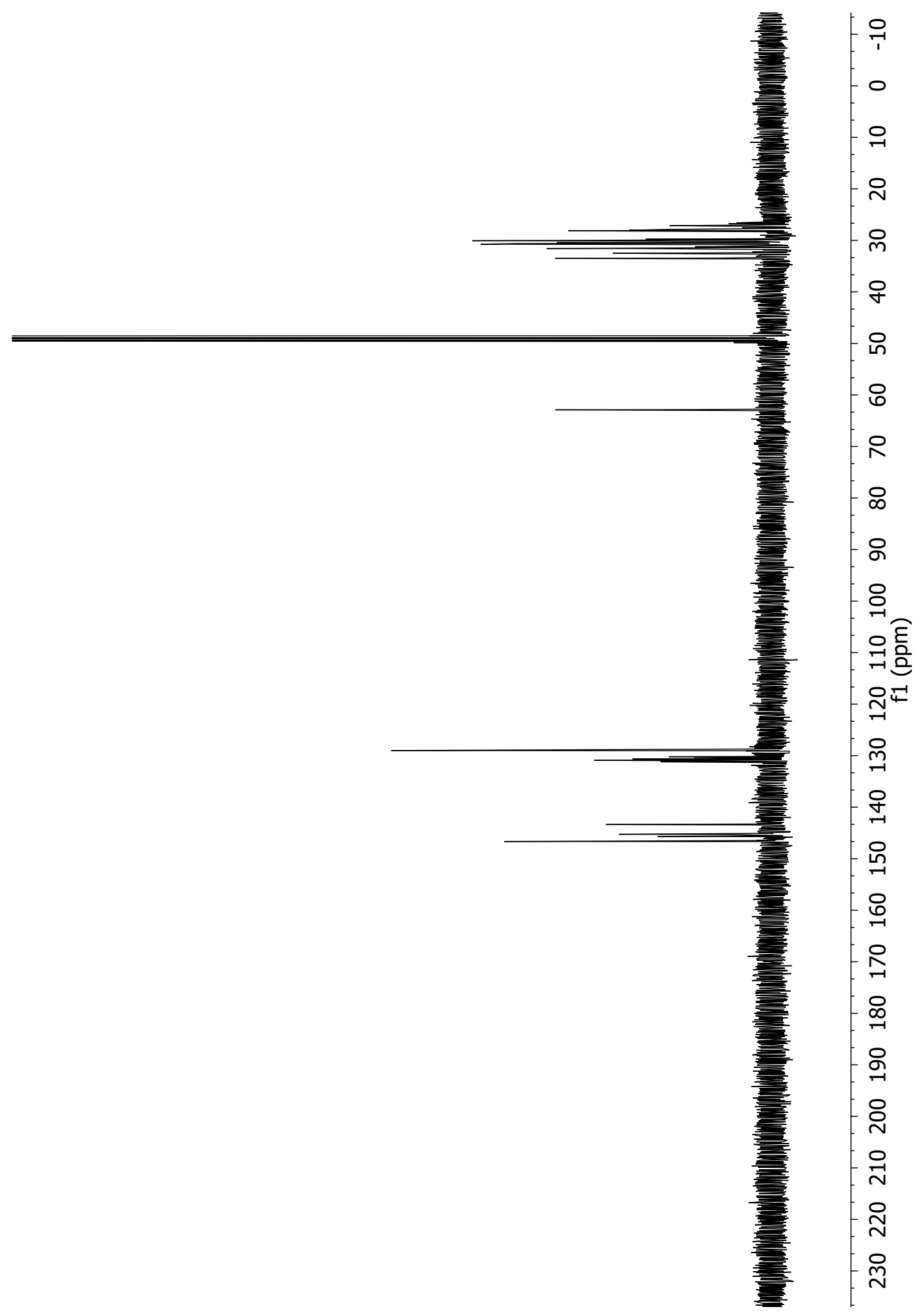

${ }^{13} \mathrm{C}$ NMR spectrum of the 3-APA VD4_47A $\left(150 \mathrm{MHz}, \mathrm{CD}_{3} \mathrm{OD}\right)$ 


\section{D.2 Fatty Acid VD3_98G}

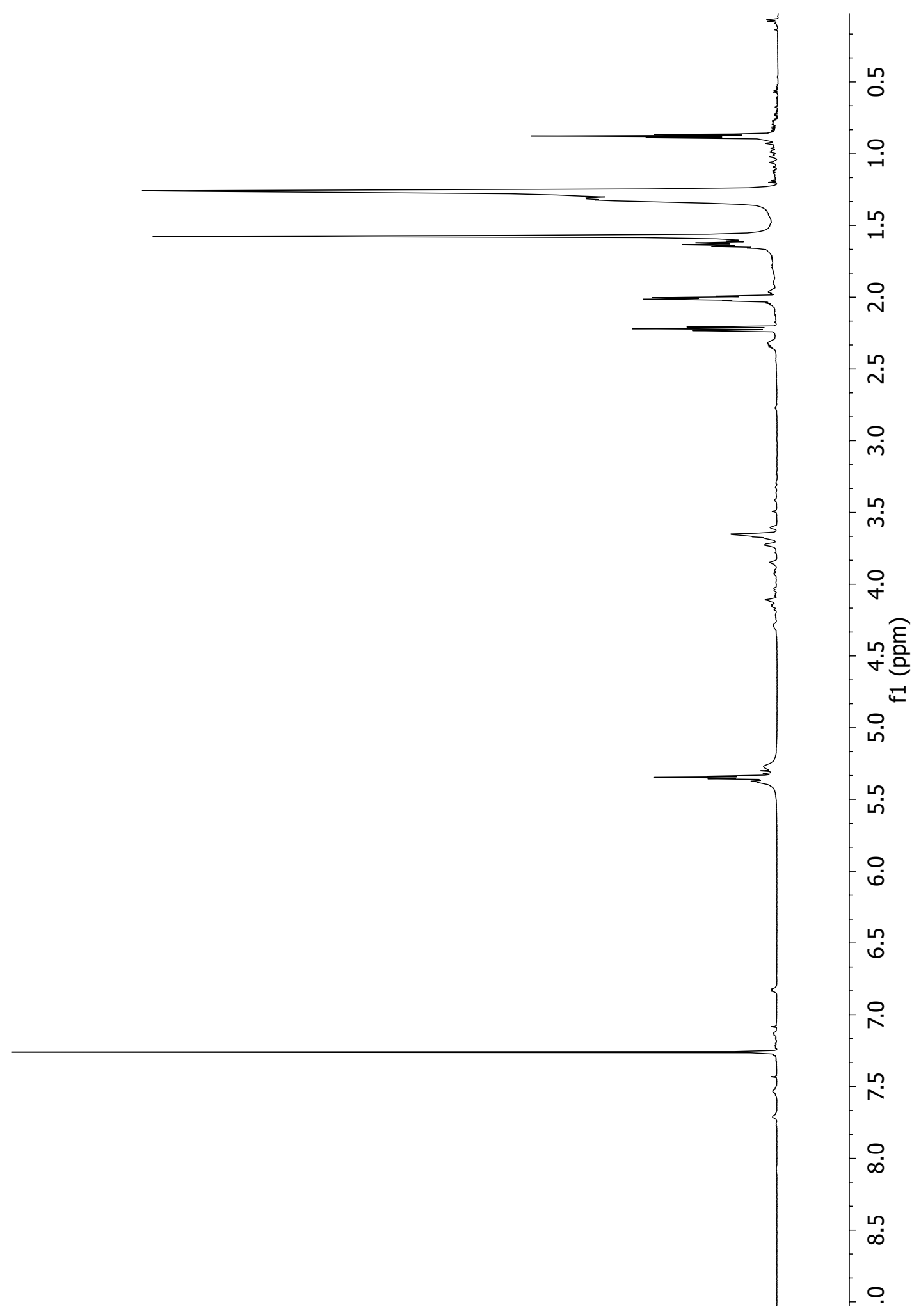

${ }^{1} \mathrm{H}$ NMR spectrum of the fatty acid VD3_98G $\left(600 \mathrm{MHz}, \mathrm{CDCl}_{3}\right)$ 


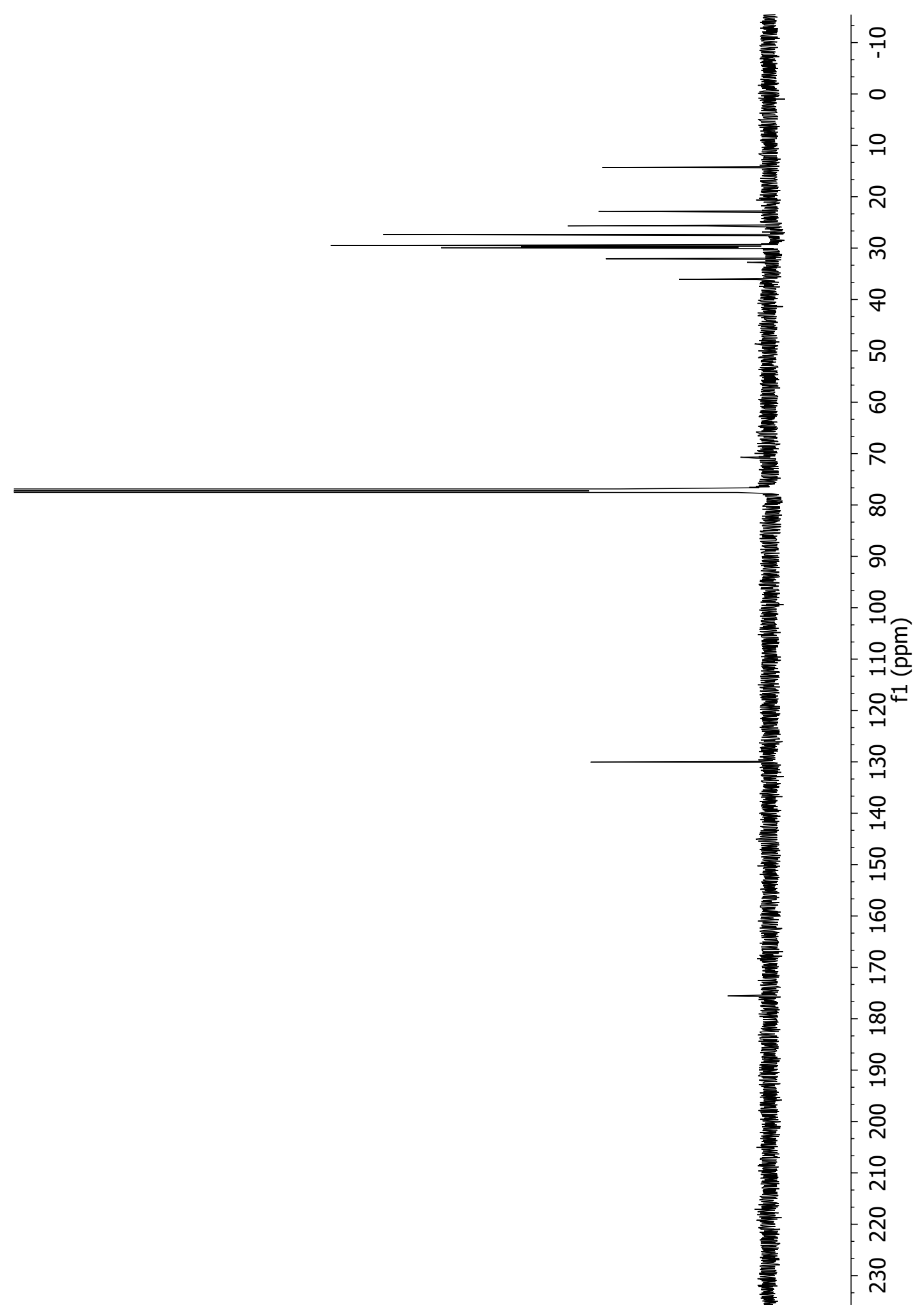

${ }^{13} \mathrm{C}$ NMR spectrum of fatty acid VD3_98G $\left(150 \mathrm{MHz}, \mathrm{CDCl}_{3}\right)$ 


\section{D.3 Phthalate Ester VD4_17B}

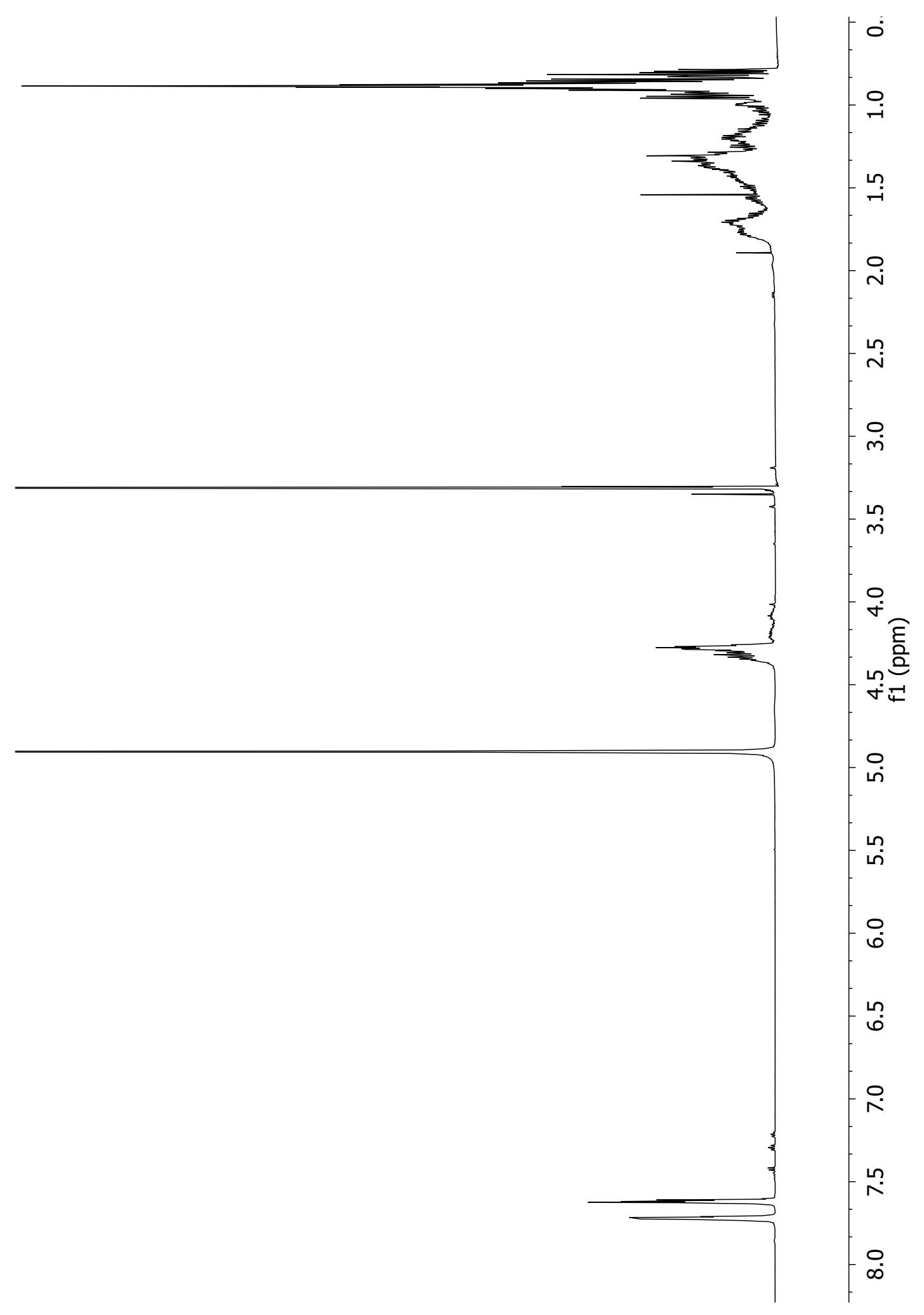

${ }^{1} \mathrm{H}$ NMR spectrum of phthalate ester mixture VD4_17B $\left(600 \mathrm{MHz}, \mathrm{CD}_{3} \mathrm{OD}\right)$ 


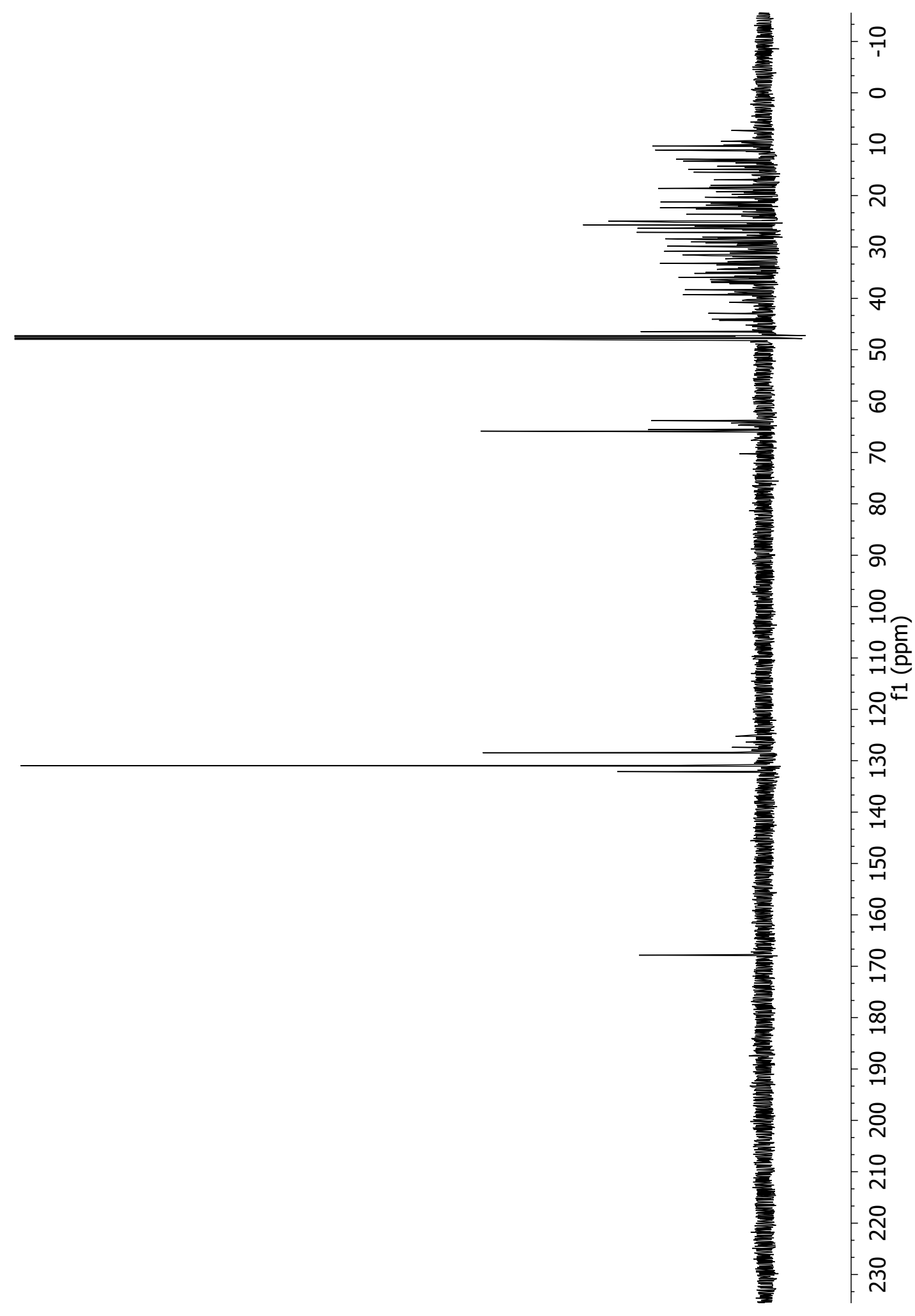

${ }^{13} \mathrm{C}$ NMR spectrum of phthalate ester mixture VD4_17B $\left(150 \mathrm{MHz}, \mathrm{CD}_{3} \mathrm{OD}\right)$ 


\section{Bibliography}

1. Newman, D. J.; Cragg, G. M.; Snader, K. M. Nat. Prod. Rep. 2000, 17, 215-234.

2. Molinski, T. F.; Dalisay, D. S.; Lievens, S. L.; Saludes, J. P. Nat. Rev. Drug Discovery 2009, 8, 69-85.

3. Scheffler, R.; Colmer, S.; Tynan, H.; Demain, A.; Gullo, V. Appl. Microbiol. Biotechnol. 2013, 97, 969-978.

4. Imhoff, J. F.; Labes, A.; Wiese, J. Biotechnol. Adv. 2011, 29, 468-482.

5. Cragg, G. M.; Grothaus, P. G.; Newman, D. J. Chem. Rev. 2009, 109, 3012-3043.

6. Thakur, N. L.; Jain, R.; Natalio, F.; Hamer, B.; Thakur, A. N.; Müller, W. E. G. Biotechnol. Adv. 2008, 26, 233-245.

7. Blunt, J. W.; Copp, B. R.; Keyzers, R. A.; Munro, M. H.; Prinsep, M. R. Nat. Prod. Rep. 2012, 29, 144-222.

8. Blunt, J. W.; Copp, B. R.; Keyzers, R. A.; Munro, M. H.; Prinsep, M. R. Nat. Prod. Rep. 2013, 30, 237-323.

9. Proksch, P. P.; Edrada, R. E.; Ebel, R. E. Appl. Microbiol. Biotechnol. 2002, 59, 125-134.

10. Newman, D. J.; Cragg, G. M. J. Nat. Prod. 2004, 67, 1216-1238.

11. Mayer, A. M. S.; Glaser, K. B.; Cuevas, C.; Jacobs, R. S.; Kem, W.; Little, R. D.; McIntosh, J. M.; Newman, D. J.; Potts, B. C.; Shuster, D. E. Trends Pharmacol. Sci. 2010, 31, 255-265.

12. European Medicines Agency: EMEA/772122/2009 - Prialt EPAR summary for the public, http://www.ema.europa.eu/docs/en_GB/document_library/EPAR_Summary_for_the public/human/000551/WC500041924.pdf, accessed at 22 April 2013.

13. United States Food and Drug Administration: FDA News Release, http://www.fda.gov/NewsEvents/Newsroom/PressAnnouncements/ucm233863.htm, accessed at 11 April 2013.

14. Jackson, K. L.; Henderson, J. A.; Phillips, A. J. Chem. Rev. 2009, 109, 3044-3079.

15. Pawlik, J. R. Chem. Rev. 1993, 93, 1911-1922.

16. Ebada, S. S.; Edrada, R. A.; Lin, W.; Proksch, P. Nat. Protoc. 2008, 3, 1820-1831.

17. Breton, R. C.; Reynolds, W. F. Nat. Prod. Rep. 2013, 30, 501-524.

18. Systema Porifera: A Guide to the Classification of Sponges, 1st ed.; Hooper, J. N. A., Soest, R. W. M. V., Eds.; Klewer Academic/Plenum: New York, 2002; Vol. 1.

19. Lipp, H.-P. Mycoses 2008, 51, 7-18.

20. Kovacic, P.; Cooksy, A. MedChemComm 2012, 3, 274-280.

21. Fleck, C. B.; Schbel, F.; Brock, M. Int. J. Med. Microbiol. 2011, 301, 400-407. 
22. Shoham, S.; Marwaha, S. J. Intensive Care Med. 2010, 25, 78-92.

23. Chuang-Stein, C.; Heft, S.; Koury, K. Pharm. Stat. 2005, 4, 253-265.

24. Fisher, M. C.; Henk, D. A.; Briggs, C. J.; Brownstein, J. S.; Madoff, L. C.; McCraw, S. L.; Gurr, S. J. Nature 2012, 484, 186-194.

25. Hube, B. Curr. Opin. Microbiol. 2004, 7, 336-341.

26. Soll, D. R. Acta Trop. 2002, 81, 101-110.

27. Hoehamer, C. F.; Cummings, E. D.; Hilliard, G. M.; Rogers, P. D. Antimicrob. Agents Chemother. 2010, 54, 1655-1664.

28. Pennisi, E. Science 2010, 327, 804-805.

29. Yoon, M.-Y.; Cha, B.; Kim, J.-C. Plant Pathol. J. 2013, 29, 1-9.

30. Blehert, D. S.; Hicks, A. C.; Behr, M.; Meteyer, C. U.; Berlowski-Zier, B. M.; Buckles, E. L.; Coleman, J. T. H.; Darling, S. R.; Gargas, A.; Niver, R.; Okoniewski, J. C.; Rudd, R. J.; Stone, W. B. Science 2009, 323, 227.

31. Frick, W. F.; Pollock, J. F.; Hicks, A. C.; Langwig, K. E.; Reynolds, D. S.; Turner, G. G.; Butchkoski, C. M.; Kunz, T. H. Science 2010, 329, 679-682.

32. Lorch, J. M.; Meteyer, C. U.; Behr, M. J.; Boyles, J. G.; Cryan, P. M.; Hicks, A. C.; Ballmann, A. E.; Coleman, J. T. H.; Redell, D. N.; Reeder, D. M.; Blehert, D. S. Nature 2011, 480, 376-378.

33. Boyles, J. G.; Cryan, P. M.; McCracken, G. F.; Kunz, T. H. Science 2011, 332, $41-42$.

34. Wenzel, R. P. N. Engl. J. Med. 2004, 351, 523-526.

35. Breithaupt, H. Nat. Biotechnol. 1999, 17, 1165-1169.

36. Fischbach, M. A.; Walsh, C. T. Science 2009, 325, 1089-1093.

37. Payne, D. J. Science 2008, 321, 1644-1645.

38. Klein, E.; Smith, D. L.; Laxminarayan, R. Emerg. Infect. Dis. 2007, 13, 1840-1846.

39. Cosgrove, S. E.; Sakoulas, G.; Perencevich, E. N.; Schwaber, M. J.; Karchmer, A. W.; Carmeli, Y. Clin. Infect. Dis. 2003, 36, 53-59.

40. Centers for Disease Control and Prevention: MRSA Infections - Definition of MRSA, http://www.cdc.gov/mrsa/definition/index.html, accessed at 23 April 2013.

41. Lowy, F. D. J. Clin. Invest. 2003, 111, 1265-1273.

42. Lim, D.; Strynadka, N. C. Nat. Struct. Biol. 2002, 9, 870-876.

43. Chambers, H. F. N. Engl. J. Med. 2005, 352, 1485-1487.

44. Fridkin, S. K.; Hageman, J. C.; Morrison, M.; Sanza, L. T.; Como-Sabetti, K.; Jernigan, J. A.; Harriman, K.; Harrison, L. H.; Lynfield, R.; Farley, M. M. N. Engl. J. Med. 2005, 352, 1436-1444. 
45. Liu, C.; Bayer, A.; Cosgrove, S. E.; Daum, R. S.; Fridkin, S. K.; Gorwitz, R. J.; Kaplan, S. L.; Karchmer, A. W.; Levine, D. P.; Murray, B. E.; J. Rybak, M.; Talan, D. A.; Chambers, H. F. Clin. Infect. Dis. 2011, 52, e18-e55.

46. Centers for Disease Control and Prevention: MRSA Infections - MRSA Statistics, http://www.cdc.gov/mrsa/statistics/index.html, accessed at 23 April 2013.

47. Spížek, J.; Novotná, J.; Řezanka, T.; Demain, A. L. J. Ind. Microbiol. Biotechnol. 2010, 37, 1241-1248.

48. Spellberg, B.; Guidos, R.; Gilbert, D.; Bradley, J.; Boucher, H. W.; Scheld, W. M.; Bartlett, J. G.; Edwards, J.; the Infectious Diseases Society of America, Clin. Infect. Dis. 2008, 46, 155-164.

49. Levina, N.; Totemeyer, S.; Stokes, N. R.; Louis, P.; Jones, M. A.; Booth, I. R. EMBO J. 1999, 18, 1730-1737.

50. Booth, I. R.; Edwards, M. D.; Black, S.; Schumann, U.; Miller, S. Nat. Rev. Microbiol. 2007, 5, 431-440.

51. Hamill, O. P.; Martinac, B. Physiol. Rev. 2001, 81, 685-740.

52. Berrier, C.; Besnard, M.; Ajouz, B.; Coulombe, A.; Ghazi, A. J. Membr. Biol. 1996, $151,175-187$.

53. Berrier, C.; Coulombe, A.; Szabo, I.; Zoratti, M.; Ghazi, A. Eur. J. Biochem. 1992, 206, 559-565.

54. Martinac, B.; Buechner, M.; Delcour, A. H.; Adler, J.; Kung, C. Proc. Natl. Acad. Sci. U. S. A. 1987, 84, 2297-2301.

55. Morris, C. E. J. Membr. Biol. 1990, 113, 93-107.

56. Kung, C.; Martinac, B.; Sukharev, S. Annu. Rev. Microbiol. 2010, 64, 313-329.

57. Hamill, O. P.; McBride, J., Don W. Amer. Sci. 1995, 83, 30-37.

58. Single-Channel Recording, 2nd Ed.; Sakmann, B., Neher, E., Eds.; Plenum Press, 1995.

59. Sukharev, S. I.; Martinac, B.; Arshavsky, V. Y.; Kung, C. Biophys. J. 1993, 65, 177-183.

60. Hamill, O. P.; Marty, A.; Neher, E.; Sakmann, B.; Sigworth, F. J. Pflügers Arch. 1981, 391, 85-100.

61. Sukharev, S. I.; Sigurdson, W. J.; Kung, C.; Sachs, F. J. Gen. Physiol. 1999, 113, 525-540.

62. Moe, P. C.; Blount, P.; Kung, C. Mol. Microbiol. 1998, 28, 583-592.

63. Perozo, E. Nat. Rev. Mol. Cell Biol. 2006, 7, 109-119.

64. Felle, H.; Porter, J. S.; Slayman, C. L.; Kaback, H. R. Biochemistry 1980, 19, 35853590 .

65. Edwards, M. D.; Black, S.; Rasmussen, T.; Rasmussen, A.; Stokes, N. R.; Stephen, T.-L.; Miller, S.; Booth, I. R. Channels 2012, 6, 272-281. 
66. Sukharev, S. I.; Blount, P.; Martinac, B.; Blattner, F. R.; Kung, C. Nature 1994, 368, 265-268.

67. Chang, G.; Spencer, R. H.; Lee, A. T.; Barclay, M. T.; Rees, D. C. Science 1998, 282, 2220-2226.

68. Pivetti, C. D.; Yen, M.-R.; Miller, S.; Busch, W.; Tseng, Y.-H.; Booth, I. R.; Saier, M. H., Jr. Microbiol. Mol. Biol. Rev. 2003, 67, 66-85.

69. Kumánovics, A.; Levin, G.; Blount, P. FASEB J. 2002, 16, 1623-1629.

70. Martinac, B.; Kloda, A. Prog. Biophys. Mol. Biol. 2003, 82, 11-24.

71. Steinbacher, S.; Bass, R.; Strop, P.; Rees, D. C. Curr. Top. Membr. 2007, 58, 1-24.

72. Cruickshank, C. C.; Minchin, R. F.; Le Dain, A. C.; Martinac, B. Biophys. J. 1997, 73, 1925-1931.

73. Research Collaboratory for Structural Bioinformatics (RCSB) Protein Data Bank, Structure ID: 2OAR, http://www.rcsb.org/pdb/explore/explore.do? structureId=2OAR, accessed at 30 April 2013.

74. Sayle, R.; Milner-White, E. J. Trends Biochem. Sci. 1995, 20, 374-376.

75. Bernstein, H. J. Trends Biochem. Sci. 2000, 25, 453-455.

76. Liu, Z.; Gandhi, C. S.; Rees, D. C. Nature 2009, 461, 120-124.

77. Dorwart, M. R.; Wray, R.; Brautigam, C. A.; Jiang, Y.; Blount, P. PLoS Biol. 2010, $8, \mathrm{e} 1000555$.

78. Iscla, I.; Wray, R.; Blount, P. Protein Sci. 2011, 20, 1638-1642.

79. Kung, C. Nature 2005, 436, 647-654.

80. Perozo, E.; Kloda, A.; Cortes, D. M.; Martinac, B. Nat. Struct. Biol. 2002, 9, 696703.

81. Bass, R. B.; Strop, P.; Barclay, M.; Rees, D. C. Science 2002, 298, 1582-1587.

82. Martinac, B.; Adler, J.; Kung, C. Nature 1990, 348, 261-263.

83. Mika, J. T.; Birkner, J. P.; Poolman, B.; Koçer, A. FASEB J. 2013, 27, 882-892.

84. Liang, C.; Louhivuori, M.; Marrink, S. J.; Jansen, T. L. C.; Knoester, J. J. Phys. Chem. Lett. 2013, 4, 448-452.

85. Yang, L.-M.; Wray, R.; Parker, J.; Wilson, D.; Duran, R. S.; Blount, P. ACS Nano 2012, 6, 1134-1141.

86. Anishkin, A.; Chiang, C.-S.; Sukharev, S. J. Gen. Physiol. 2005, 125, 155-170.

87. Birkner, J. P.; Poolman, B.; Koçer, A. Proc. Natl. Acad. Sci. U. S. A. 2012, 109, 12944-12949.

88. Deplazes, E.; Louhivuori, M.; Jayatilaka, D.; Marrink, S. J.; Corry, B. PLoS Comput. Biol. 2012, 8, 1-15. 
89. Rui, H.; Kumar, R.; Im, W. Biophys. J. 2011, 101, 671-679.

90. Booth, I. R.; Blount, P. J. Bacteriol. 2012, 194, 4802-4809.

91. Haswell, E.; Phillips, R.; Rees, D. Structure 2011, 19, 1356-1369.

92. Iscla, I.; Blount, P. Biophys. J. 2012, 103, 169-174.

93. Martinac, B. J. Cell Sci. 2004, 117, 2449-2460.

94. Nomura, T.; Cranfield, C. G.; Deplazes, E.; Owen, D. M.; Macmillan, A.; Battle, A. R.; Constantine, M.; Sokabe, M.; Martinac, B. Proc. Natl. Acad. Sci. U. S. A. 2012, 109, 8770-8775.

95. Datta, S.; Zhou, Y.-D.; Nagle, D. G. J. Nat. Prod. 2013, 76, 642-647.

96. Hoang, V. L.; Li, Y.; Kim, S.-K. Bioorg. Med. Chem. Lett. 2008, 18, 2083-2088.

97. Li, Y.; Qian, Z.-J.; Kim, S.-K. Bioorg. Med. Chem. Lett. 2008, 18, 6130-6134.

98. Ciminiello, P.; Fattorusso, E.; Magno, S.; Mangoni, A.; Pansini, M. J. Am. Chem. Soc. 1990, 112, 3505-3509.

99. Cafieri, F.; Fattorusso, E.; Gavagnin, M.; Santacroce, C. J. Nat. Prod. 1985, 48, 944-947.

100. Notaro, G.; Piccialli, V.; Sica, D.; Corriero, G. J. Nat. Prod. 1991, 54, 1570-1575.

101. Luo, X.; Li, F.; Shinde, P. B.; Hong, J.; Lee, C.-O.; Im, K. S.; Jung, J. H. J. Nat. Prod. 2006, 69, 1760-1768.

102. Piccialli, V.; Sica, D. J. Nat. Prod. 1987, 50, 915-920.

103. Grage, S. L. et al. Biophys. J. 2011, 100, 1252-1260.

104. Schmitz, F. J.; Hollenbeak, K. H.; Campbell, D. C. J. Org. Chem. 1978, 43, 39163922.

105. Turk, T.; Sepčić, K.; Mancini, I.; Guella, G. 3-Alkylpyridinium and 3-alkylpyridine compounds from marine sponges, their synthesis, biological activities and potential use. In Bioactive Natural Products (Part O); Atta-ur-Rahman,, Ed.; Elsevier, 2008; Vol. 35, pp $355-397$.

106. Van Soest, R. W. M.; Boury-Esnault, N.; Hooper, J. N. A.; Rützler, K.; de Voogd, N. J.; Alvarez de Glasby, B.; Hajdu, E.; Pisera, A. B.; Manconi, R.; Schoenberg, C.; Janussen, D.; Tabachnick, K. R.; Klautau, M.; Picton, B.; Kelly, M.; Vacelet, J.; Dohrmann, M.; Cristina Díaz, M., World Porifera database. http://www.marinespecies.org/porifera, accessed at 04 June 2013.

107. Stierle, D. B.; Faulkner, D. J. J. Nat. Prod. 1991, 54, 1134-1136.

108. Talpir, R.; Rudi, A.; Ilan, M.; Kashman, Y. Tetrahedron Lett. 1992, 33, 3033-3034.

109. Buchanan, M. S.; Carroll, A. R.; Addepalli, R.; Avery, V. M.; Hooper, J. N. A.; Quinn, R. J. J. Nat. Prod. 2007, 70, 2040-2041.

110. Volk, C. A.; Köck, M. Org. Biomol. Chem. 2004, 2, 1827-1830. 
111. Schmidt, G.; Timm, C.; Grube, A.; Volk, C. A.; Köck, M. Chem.-Eur. J. 2012, 18, 8180-8189.

112. Laville, R.; Thomas, O. P.; Berrue, F.; Reyes, F.; Amade, P. Eur. J. Org. Chem. 2008, 2008, 121-125.

113. Anan, H.; Seki, N.; Noshiro, O.; Honda, K.; Yasumuro, K.; Ozasa, T.; Fusetani, N. Tetrahedron 1996, 52, 10849-10860.

114. Kaiser, A.; Billot, X.; Gateau-Olesker, A.; Marazano, C.; Das, B. C. J. Am. Chem. Soc. 1998, 120, 8026-8034.

115. Timm, C.; Volk, C.; Sasse, F.; Köck, M. Org. Biomol. Chem. 2008, 6, 4036-4040.

116. Schmidt, G.; Timm, C.; Köck, M. Z. Naturforsch., B: J. Chem. Sci. 2011, 66, 745748.

117. Fusetani, N.; Asai, N.; Matsunaga, S.; Honda, K.; Yasumuro, K. Tetrahedron Lett. 1994, 35, 3967-3970.

118. Oku, N.; Nagai, K.; Shindoh, N.; Terada, Y.; van Soest, R. W.; Matsunaga, S.; Fusetani, N. Bioorg. Med. Chem. Lett. 2004, 14, 2617-2620.

119. de Oliveira, J. H. H. L.; Grube, A.; Köck, M.; Berlinck, R. G. S.; Macedo, M. L.; Ferreira, A. G.; Hajdu, E. J. Nat. Prod. 2004, 67, 1685-1689.

120. de Oliveira, J. H. H. L.; Seleghim, M. H. R.; Timm, C.; Grube, A.; Köck, M.; Nascimento, G. G.; Martins, A. C. T.; Silva, E. G. O.; De Souza, A. O.; Minarini, P. R. R.; Galetti, F. C. S.; Silva, C. L.; Hajdu, E.; Berlinck, R. G. S. Mar. Drugs 2006, $4,1-8$.

121. Ondeyka, J. G. et al. Mol. Divers. 2005, 9, 123-129.

122. Teruya, T.; Kobayashi, K.; Suenaga, K.; Kigoshi, H. J. Nat. Prod. 2006, 69, 135137.

123. Xu, N. J.; Sun, X.; Yan, X. J. Chin. Chem. Lett. 2007, 18, 947-950.

124. Casapullo, A.; Pinto, O. C.; Marzocco, S.; Autore, G.; Riccio, R. J. Nat. Prod. 2009, 72, 301-303.

125. Laville, R.; Genta-Jouve, G.; Urda, C.; Fernández, R.; Thomas, O. P.; Reyes, F.; Amade, P. Molecules 2009, 14, 4716-4724.

126. Lee, Y.; Jang, K. H.; Jeon, J.-e.; Yang, W.-Y.; Sim, C. J.; Oh, K.-B.; Shin, J. Mar. Drugs 2012, 10, 2126-2137.

127. Grube, A.; Timm, C.; Köck, M. Eur. J. Org. Chem. 2006, 2006, 1285-1295.

128. Schmidt, G.; Timm, C.; Köck, M. Org. Biomol. Chem. 2009, 7, 3061-3064.

129. Volk, C. A.; Köck, M. Org. Lett. 2003, 5, 3567-3569.

130. Rodenko, B.; Al-Salabi, M. I.; Teka, I. A.; Ho, W.; El-Sabbagh, N.; Ali, J. A. M.; Ibrahim, H. M. S.; Wanner, M. J.; Koomen, G.-J.; de Koning, H. P. ACS Med. Chem. Lett. 2011, 2, 901-906.

131. de Haan, J. W.; Van de Ven, L. J. M. Org. Magn. Reson. 1973, 5, 147-153. 
132. Nguyen, T. H.; Um, B. H.; Kim, S. M. J. Food Sci. 2011, 76, H208-H214.

133. Gunstone, F. D. J. Am. Oil Chem. Soc. 1993, 70, 965-970.

134. Wang, G.-Y.-S.; Kuramoto, M.; Uemura, D.; Yamada, A.; Yamaguchi, K.; Yazawa, K. Tetrahedron Lett. 1996, 37, 1813-1816.

135. Laville, R.; Amade, P.; Thomas, O. P. Pure Appl. Chem. 2009, 81, 1033-1040.

136. Davies-Coleman, M. T.; Faulkner, D. J.; Dubowchik, G. M.; Roth, G. P.; Polson, C.; Fairchild, C. J. Org. Chem. 1993, 58, 5925-5930.

137. Timm, C.; Mordhorst, T.; Köck, M. Mar. Drugs 2010, 8, 483-497.

138. Silverstein, R. M.; Webster, F. X.; Kiemle, D. J. Spectrometric Identification of Organic Compounds, 7th Ed.; John Wiley \& Sons, Inc., 2005.

139. Djerassi, C.; Engle, R. R. J. Am. Chem. Soc. 1953, 75, 3838-3840.

140. Williams, D. E.; Keyzers, R. A.; Warabi, K.; Desjardine, K.; Riffell, J. L.; Roberge, M.; Andersen, R. J. J. Org. Chem. 2007, 72, 9842-9845.

141. Carlsen, P. H. J.; Katsuki, T.; Martin, V. S.; Sharpless, K. B. J. Org. Chem. 1981, 46, 3936-3938.

142. West, L. M. The Isolation of Secondary Metabolites from New Zealand Marine Sponges, Ph.D. thesis, Victoria University of Wellington, 2001.

143. GraphPad Prism version 5.00 for Windows, GraphPad Software, San Diego California USA, www.graphpad.com. 